\title{
Modelling and Synthesis of High Speed Craft Acceleration Profiles
}

by

\section{Mohamed El Tayeby}

\author{
A thesis submitted to \\ the Faculty of Graduate and Postdoctoral Affairs \\ in partial fulfilment of \\ the requirements for the degree of \\ Master of Applied Science \\ in \\ Mechanical Engineering
}

Mechanical and Aerospace Engineering

Carleton University

Ottawa, Ontario, Canada

January 2015

Copyright (C)

2015 - Mohamed El Tayeby 
The undersigned recommend to the Faculty of Graduate and Postdoctural Affairs acceptance of the thesis

\title{
Modelling and Synthesis of High Speed Craft Acceleration Profiles
}

\author{
Submitted by Mohamed El Tayeby \\ in partial fulfilment of the requirements for the degree of \\ Master of Applied Science
}

Dr. Robert Langlois, Supervisor

Dr. Fred Afagh, Co-Supervisor

Dr. Metin Yaras, Department Chair

Carleton University

2015 


\section{Abstract}

High Speed Craft (HSC) occupants are subjected to a harsh environment characterized by vibration and repeated shock. Significant research has therefore been invested into HSC shock mitigation. The objective of this work was to determine standard shock acceleration profiles for HSC based on sea trial data. The knowledge of real-life sea inputs is important for in-lab testing of HSC shock mitigation seats. Sea trial vertical acceleration data of a Rigid-Hull Inflatable Boat was analyzed with the aim of characterizing shock acceleration pulses experienced by the boat. Five common types of shock acceleration profiles were identified. These profiles were represented by a combination of modified Gaussian curves. The statistical characteristics of the observed pulses were obtained by curve fitting the data. An algorithm employing copulas and non-parametric density estimation was developed for generating synthetic time histories which have the same statistical characteristics as the observed data. 


\section{Acknowledgments}

I would like to express my regards and thanks to my supervisors, Dr. Robert Langlois and Dr. Fred Afagh, for their invaluable guidance and for being always available for consultation throughout the course of this project. I would like to thank Defence Research and Development Canada-Atlantic and all funding parties for providing this research opportunity. Thanks also go to the members of the research team I was part of, for their help during the course of this project. I am grateful to my family for their always-present support. Special thanks to my dad for his heavy moral support and invaluable advice. 


\section{Table of Contents}

$\begin{array}{ll}\text { Abstract } & \text { iii }\end{array}$

Acknowledgments $\quad$ iv

Table of Contents $\quad$ v

List of Tables viii

List of Figures $\quad$ x

List of Acronyms xiii

1 Introduction 1

1.1 Literature Review . . . . . . . . . . . . . . . . . . . . . 3

1.2 Thesis Objectives . . . . . . . . . . . . . . . . 6

1.3 Thesis Outline . . . . . . . . . . . . . . . . 7

2 Sea Trial Data Analysis and Standard Acceleration Profiles 8

2.1 Data Processing . . . . . . . . . . . . . . . . . 9

2.1.1 Accelerometer Gravity Offset . . . . . . . . . . . . . . 10 
2.1 .2 Filtering . . . . . . . . . . . . . . . . . 11

2.1 .3 Impact Pulse Extraction . . . . . . . . . . . . . . . . 12

2.2 Acceleration Profiles . . . . . . . . . . . . . . . . . . . . . 13

2.2 .1 Type 1: Half-Sine . . . . . . . . . . . . . . . . . . 14

2.2.2 Type 2: Half-Sine with Single Shoulder . . . . . . . . . 16

2.2.3 Type 3: Half-Sine with Multiple Shoulders . . . . . . . . . 18

2.2.4 Type 4: Delayed Peak . . . . . . . . . . . . . . . . . 21

2.2 .5 Type 5: Double peak . . . . . . . . . . . . . . . 23

2.3 Curve Fitting Algorithm . . . . . . . . . . . . . . . . . . . . . 26

2.3 .1 Pulse Type Detection . . . . . . . . . . . . . . . . 27

2.4 Reduction of Sea Trial Data . . . . . . . . . . . . . . 28

2.5 Discussion . . . . . . . . . . . . . . . . . . . . . . . . . 42

2.5.1 On the Curve Fitting Process $\ldots \ldots \ldots \ldots$

2.5.2 On the Results . . . . . . . . . . . . . . . . . 43

2.6 Conclusion . . . . . . . . . . . . . . . . . . . . . . . . . 44

3 Synthetic Time History Generation 45

3.1 Synthetic Pulse Generation Methods . . . . . . . . . . . . . . 46

3.1.1 Simulating Dependent Random Variables using Copulas . . 46

3.1.2 Simulating Dependent Random Variables by Non-parametric Density Estimation . . . . . . . . . . . . . . . . . 48

3.2 Inter-Pulse Characteristics $\ldots \ldots \ldots \ldots \ldots$

3.3 Results . . . . . . . . . . . . . . . . . 56

3.4 Discussion . . . . . . . . . . . . . . . . 68 
3.4.1 On Synthetic Pulses . . . . . . . . . . . . . 68

3.4 .2 On Inter-Pulse Characteristics . . . . . . . . . . . . . . 69

3.5 Conclusion . . . . . . . . . . . . . . . . . . . . . . . . 69

$\begin{array}{lll}4 \text { Conclusion } & 70\end{array}$

$\begin{array}{ll}\text { References } & 72\end{array}$

Appendix A Reduction of Additional Data Set $\quad 74$

Appendix B Data Pre-processing Code $\quad 79$

$\begin{array}{ll}\text { Appendix C Curve Fitting Algorithm } & 84\end{array}$

Appendix D Classification of Fitted Pulses into the Different Profiles $\begin{array}{ll}\text { Code } & 92\end{array}$

Appendix E Synthetic Data Generation Code 114

Appendix F Code for Obtaining Parameter Mean Values $\quad 170$ 


\section{List of Tables}

2.1 Observed Type 5 variations. . . . . . . . . . . . . . . . . 24

2.2 Pulse detection rate. . . . . . . . . . . . . . . . . . 28

2.3 Day 1 - bow parameter mean values for Types 1 to 4 profiles. . . . . 29

2.4 Day 1 - bow parameter mean values for Type 5 Profiles. . . . . . . . 30

2.5 Day 2 - bow parameter mean values Types 1 to 4 Profiles. . . . . . . 31

2.6 Day 2 - bow parameter mean values for Type 5 Profiles. . . . . . . . 32

2.7 Day 3 - bow parameters mean values Types 1 to 4 Profiles. . . . . . . 33

2.8 Day 3 - bow parameter mean values for Type 5 Profiles. . . . . . . . 34

2.9 Day 4 - bow parameter mean values for Types 1 to 4 Profiles. . . . . 35

2.10 Day 4 - bow parameter mean values for Type 5 Profiles. . . . . . . . 36

3.1 Parameter mean values comparison for Types 1 through 4 - copula method. . . . . . . . . . . . . . . . 63

3.2 Parameter mean values comparison for Types 1 through 4 - density estimation method. . . . . . . . . . . . . . . . . . . . 64

3.3 Parameter mean values comparison for Type 5 profiles - copula method. 65

3.4 Parameter mean values comparison for Type 5 profiles - density esti-

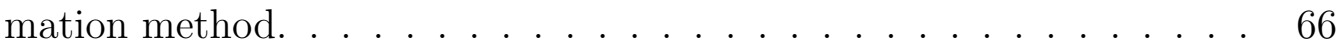

viii 
3.5 Prescribed vs detected profiles. . . . . . . . . . . . . . 67

A.1 Forward position - parameter mean values for Types 1 to 4 profiles. . 77

A.2 Forward position - parameter mean values for Type 5 profiles. . . . . 78 


\section{List of Figures}

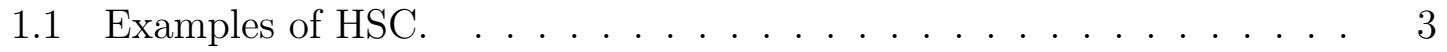

1.2 Sample of RHIB vertical acceleration time history. . . . . . . . . 5

1.3 Algorithm for analyzing and simulating HSC acceleration data. . . . . 7

2.1 Wave height and period. . . . . . . . . . . . . . . . 10

2.2 Power spectral density of unfiltered vertical acceleration of the midships portion of a RHIB. . . . . . . . . . . . . . . . 11

2.3 Vertical acceleration time history of the midships portion of a RHIB. 12

2.4 Typical normalized acceleration pulse. . . . . . . . . . . . . . 13

2.5 Type 1 profile. . . . . . . . . . . . . . . . . 16

2.6 Type 2 profile. . . . . . . . . . . . . . . . . . . 18

2.7 Type $3 . I$ profile. . . . . . . . . . . . . . . . . . . . . . . 19

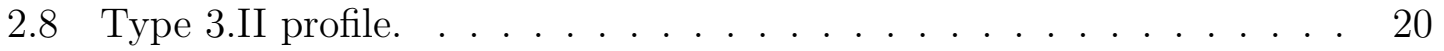

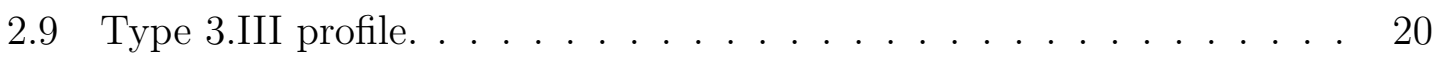

2.10 Type 4. I profile. . . . . . . . . . . . . . . . . . . . . 22

2.11 Type $4 . I I$ profile. . . . . . . . . . . . . . . . . . . 22

2.12 Type 4.III profile. . . . . . . . . . . . . . . . . . . 23

2.13 Type $1-2$ profile. . . . . . . . . . . . . . . . . 25 
2.14 Type $2-2$ profile. . . . . . . . . . . . . . . . . 25

2.15 Type 2-3.I profile. . . . . . . . . . . . . . . . . . 26

2.16 Day 1 profile distributions. . . . . . . . . . . . . 37

2.17 Distribution of Type 5 profiles for Day 1 - bow. . . . . . . . . . 37

2.18 Day 2 profile distributions. . . . . . . . . . . . . . 38

2.19 Distribution of Type 5 profiles for Day 2 - bow. . . . . . . . . . 38

2.20 Day 3 profile distributions. . . . . . . . . . . . . . . . . . . . 39

2.21 Distribution of Type 5 profiles for Day 3 - bow. . . . . . . . . . . 39

2.22 Day 4 profile distributions. . . . . . . . . . . . . . . . . 40

2.23 Distribution of Type 5 profiles for Day 4 - bow. . . . . . . . . . 40

2.24 Profiles at parameter mean values of Day 1 at the bow. . . . . . . . 41

3.1 Illustration of space discretization for two variables. . . . . . . . 50

3.2 Example of a motion cycle. . . . . . . . . . . . . . . . 54

3.3 Example of drift correction. . . . . . . . . . . . . . . . 55

3.4 Synthetic time history. . . . . . . . . . . . . . . . 57

3.5 Matrix plot of the original parameters for Type 2 (in blue) and the simulated parameters using copulas (in green). . . . . . . . . 58

3.6 Matrix plot of the original parameters for Type 2 (in blue) and simulated parameters using density estimation (in green). . . . . . . . 59

3.7 Distribution of the original parameters for Type $2 \ldots \ldots \ldots$. . . . 60

3.8 Distribution of the simulated parameters using copulas for Type 2. . . 61

3.9 Distribution of the simulated parameters using density estimation for

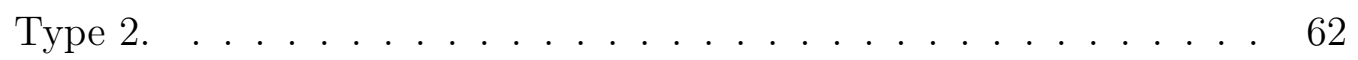

A.1 Profile distribution - forward position. . . . . . . . . 75 
A.2 Distribution of Type 5 profiles - forward position. . . . . . . . . . 76 


\section{List of Acronyms}

\begin{tabular}{ll} 
Acronyms & Definition \\
\hline \hline HSC & high speed craft \\
SMS & shock mitigation seat \\
RHIB & rigid-hull inflatable boat \\
DRDC & Defence Research and Development Canada \\
ICI & impact count index \\
\hline
\end{tabular}

xiii 


\section{Chapter 1}

\section{Introduction}

A research project investigating the dynamics of High Speed Craft (HSC) Shock Mitigation Seats (SMSs) was recently conducted by Carleton University's Applied Dynamics Lab in collaboration with Defence Research and Development Canada (DRDC) - Atlantic. The research presented in this thesis addresses one of the overall project objectives which was to determine HSC standard shock acceleration profiles based on the analysis of sea trial acceleration data. The remaining objectives of the project were to:

1. Develop 3-D dynamic models of sample SMSs that were provided by DRDC;

2. Characterize dynamic properties of seats and their components such as springs and dampers using both a drop tower and a suspension component test frame; and

3. Design and build a test rig which allows testing of seats under simulated sea-like conditions. 
The common goal of the project was to develop tools which would contribute to making an informed selection of SMSs based on the prevailing operational environment for the Canadian Forces. The SMSs which were modelled and tested are used on Rigid-Hull Inflatable Boats (RHIBs).

A HSC is generally defined as a vessel which can achieve speeds higher than 30 knots [1]. Faltinsen classifies HSC into three categories: submergedhull-supported, foil-supported, and air-cushion-supported vessels. Furthermore, submerged-hull-supported vessels are divided into semi-displacement and planing craft [1]. The work presented in this thesis pertains mostly to planing craft such as small patrol and rescue boats used by navies and coast guards (Figure 1.1). Occupants of HSC are subjected to high acceleration impacts and repeated shock which often can lead to injury [2-4]. Research into HSC shock mitigation has been undertaken in order to reduce occupant injury and discomfort during high speed transits. Three areas of study and their mutual interaction are involved in HSC shock mitigation:

1. HSC dynamics;

2. Seat dynamics; and

3. Human body dynamics.

The topic of this thesis is concerned with HSC dynamics. More specifically, the focus is on the shock motion of HSC. The overall goal of the research is to characterize HSC vertical impact acceleration pulses and to establish standard acceleration profiles based on sea trial boat data. The importance of this investigation lies in the fact that knowledge of real-life seat inputs is necessary for meaningful in-lab seat testing 


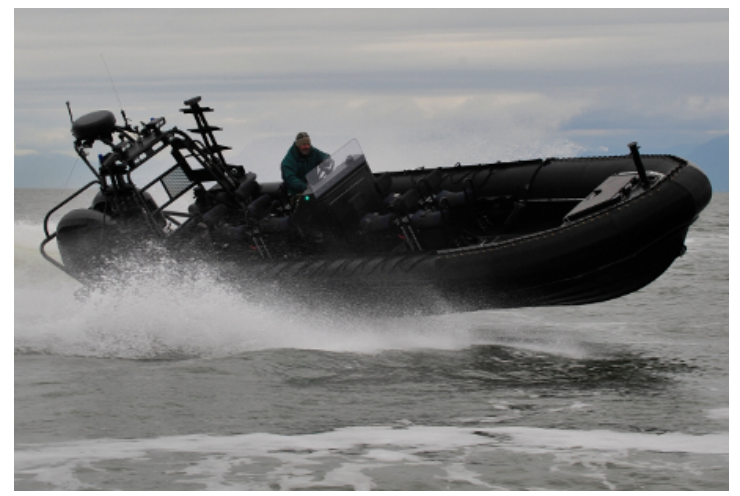

(a) DAMEN Interceptor high speed planing craft [5]

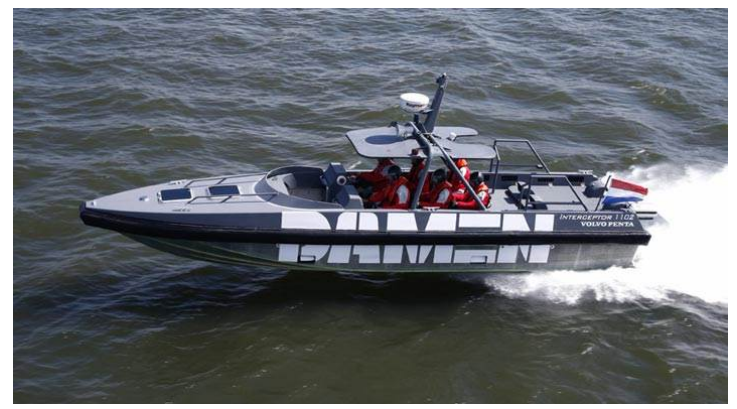

(b) Titan Boats Rigid Inflatable Boat [6]

Figure 1.1: Examples of HSC.

and simulation of seat motion. This research also opens the door for establishing correlations between boat characteristics, sea parameters, and impact profiles.

In the remainder of this chapter, Section 1.1 presents background information on HSC shock motion, biased towards planing craft motion. In Section 1.2, the thesis objectives and approach are explained. Finally, the thesis outline is presented in Section 1.3.

\section{$1.1 \quad$ Literature Review}

Much of the research on HSC dynamics has been experimental in nature. An extensive review of experimental and numerical methods for the analysis of HSC motion can be found in [7]. While the manoeuvering and seakeeping performance of low speed craft are well understood, the prediction of HSC performance, particularly of planing craft, is a challenge due to the occurrence of numerous wave slamming/impact events during high speed transits [4,8] (Figure 1.2). This type of shock loading 
increases occupant injury risk and therefore is a major concern in the study of HSC motion. Wave slamming occurs when the forward portion of the craft impacts with oncoming waves while the boat pitches and heaves about a rotation point close to the aft portion of the craft [3]. Wave slamming can also result from the entire boat leaving the water and re-entering [3].

Experiments have been conducted to study hull vertical water entry. Generally these experiments consisted of dropping wedge shaped objects into water. Numerical methods, such as CFD and smoothed particle hydrodynamics, have been used to simulate wedge water entry and have been validated against experimental test data $[1,9]$. The wedge water entry problem is a starting point for understanding hull water entry in real-life sea conditions.

Empirical formulae are available for the determination of the average impact acceleration of particular types of planing hulls in prescribed sea conditions [10]. These formulae do not, however, provide information about impact pulse shape. Besides empirical correlations, statistical measures have been developed to characterize HSC shock motion. For example, the Impact Count Index (ICI) is a measure of the number of impacts in a given vertical acceleration time history which have a maximum amplitude below a certain acceleration value. By using the ICI, a criterion can be defined which sets a shock exposure limit [11]. Another example of statistical methods applied to HSC motion is a technique which has been developed to synthesize vertical acceleration time histories for planing craft [12]. The generalized Pareto distribution was fitted to impact peak values from recorded time histories. Random numbers were then generated and converted to peak values using the Pareto distribution in 
order to produce time histories with the same peak value characteristics. An additional step was taken in which the mean of the Pareto distribution was set to the average impact acceleration predicted by the aforementioned empirical correlations, such that time histories can be obtained which are representative of different types of planing craft in different sea conditions. In this study, the impact pulses of the produced time histories were assumed to have a half-sine shape.

Statistical methods have focused so far on the peak values of HSC impact pulses. However, the pulse shape characteristics are also important since they contain information about the energy of impact. Therefore, for the purpose of testing SMSs under sea-like conditions, it is necessary to have knowledge of the impact pulse shape characteristics of HSC.

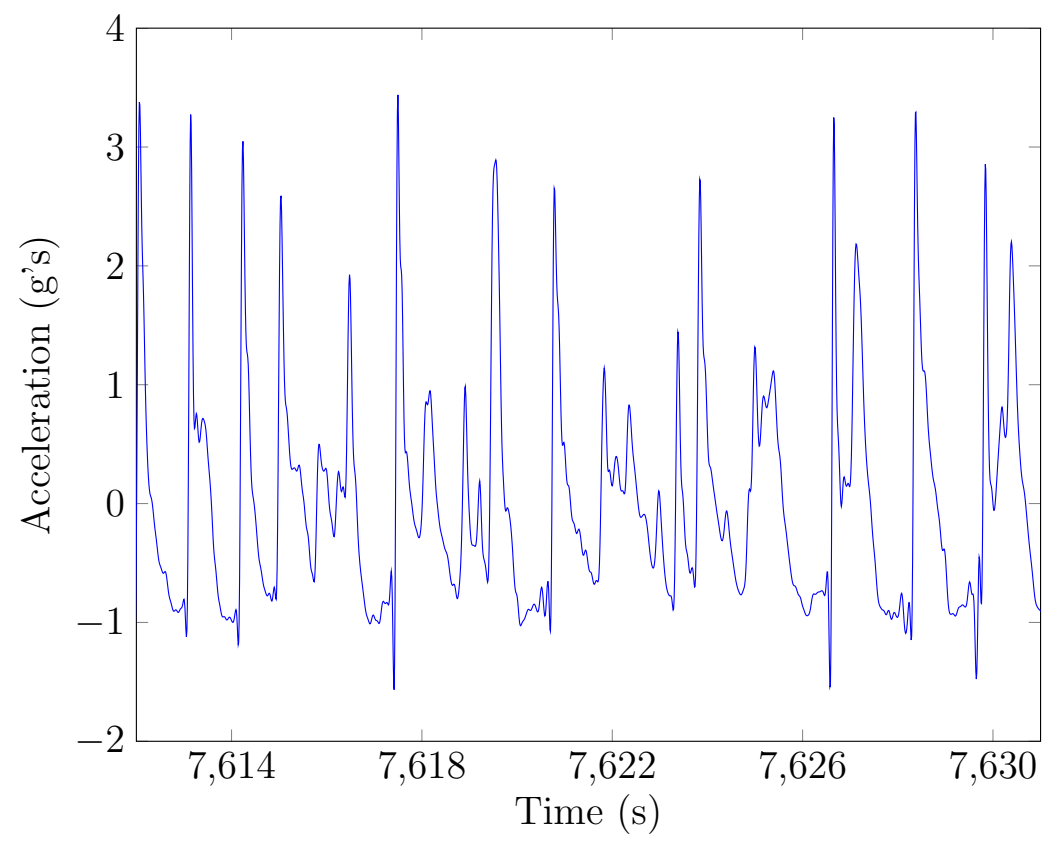

Figure 1.2: Sample of RHIB vertical acceleration time history (data provided by DRDC). 


\subsection{Thesis Objectives}

The main objective of this work was to characterize HSC vertical impact acceleration pulses. The reason for considering vertical impact accelerations is that they are responsible for spine and knee injury which are the most common injury locations. The characterization of acceleration pulses was achieved through the analysis of RHIB sea trial data. While RHIBs are one type of HSC, the presented work could potentially apply to other types of HSC. A set of equations was developed to fit the observed acceleration profiles. Based on the sea trial data, five types of acceleration profiles were distinguished. The second objective of this work was to produce acceleration time histories having the same pulse shape characteristics as those of the observed data. Two methods were implemented in order to produce correlated random variables. The first employs mathematical functions known as copulas; the second relies on a non-parameteric estimate of the joint probability density function of the variables. These methods were used to generate equation parameters and in turn to synthesize acceleration time histories.

The flow chart provided in Figure 1.3 illustrates the workings of the produced algorithm. The sea trial data is first processed in order to remove the accelerometer gravity offset and noise from the acceleration signal. The acceleration pulses are then extracted from the available time histories. These pulses are then curve-fitted with the equation form proposed in Chapter 2, using a built-in Matlab function (lsqcurvefit). From the curve fitting process, the distribution of acceleration profiles can be determined as well as equation parameters. From the acquired statistics, synthetic time histories can then be produced which have the same pulse shape 
characteristics as the original sea trial data.

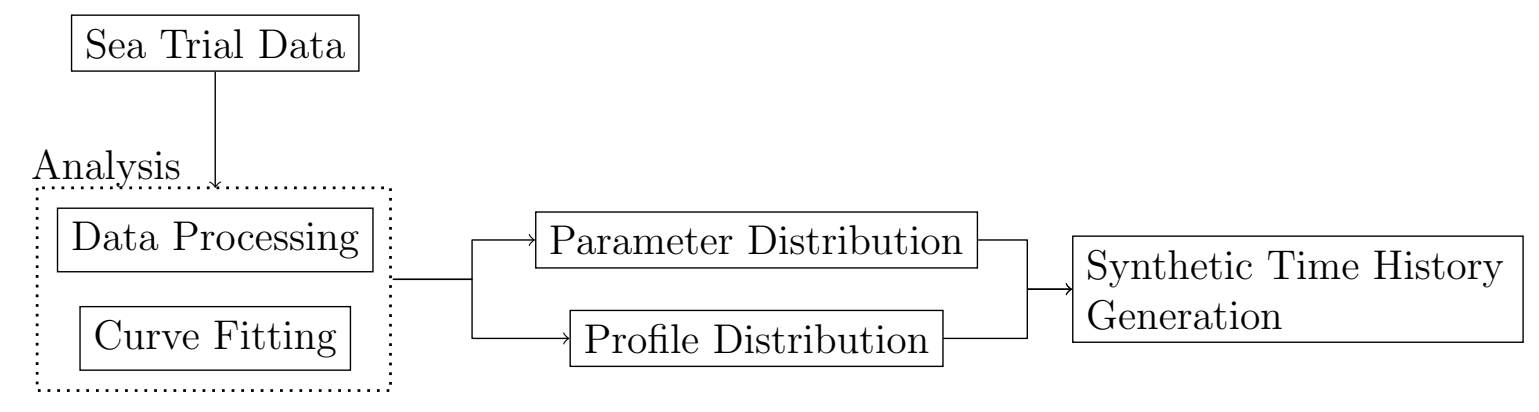

Figure 1.3: Algorithm for analyzing and simulating HSC acceleration data.

\subsection{Thesis Outline}

The remainder of this thesis is organized as follows. Chapter 2 provides a detailed description of how the original raw boat data was processed. The curve fitting equations are presented and the curve fitting process is explained. The statistical characteristics of the observed pulses, in the sea trial data provided by DRDC, are presented. A discussion follows in which comments are made on the curve fitting procedure and the results. Chapter 3 provides an explanation of the time history generation process. The statistical characteristics of a sample synthesized time history are presented. A discussion is then provided in which generated time histories are compared to the observed data. Chapter 4 summarizes the contributions of this work and provides recommendations for future work. 


\section{Chapter 2}

\section{Sea Trial Data Analysis and Standard Acceleration Profiles}

Sea trial RHIB acceleration data, provided by DRDC, was processed and analysed in order to understand the characteristics of HSC impact pulses. From a thorough inspection of the data it was postulated that impact pulses could be classified into several distinct types. An equation was sought to represent each one of those types. Ultimately, the observed pulses were classified into five main categories after an iterative process which required re-examining the data and evaluating various equation forms. A set of equations consisting of a combination of modified Gaussian curves was found to be an ideal fit for the data.

The processing of the raw data is explained in Section 2.1. The algorithm used for the curve fitting process is described in Section 2.3. The different types of acceleration pulses are presented, along with their representative equations, in Section 2.2. Results of the analysis are presented in Section 2.4. The results are discussed in Section 2.5. Concluding remarks are presented in Section 2.6. 


\subsection{Data Processing}

Data collected from a RHIB sea trial spanning five days (June $11^{\text {th }}-15^{\text {th }}, 2007$ ) was provided to the research team. The variation of wave height and modal period over the duration of the trial are shown in Figure 2.1. Two data acquisition packages were mounted on the longitudinal centreline of the RHIB with one at midships and one at the bow of the boat. Each package contained:

- a Crossbow CXL-HF Series \pm 10 g accelerometer (sampling rate of $2000 \mathrm{~Hz}$ );

- a Crossbow CXL-HF Series \pm 100 g accelerometer (sampling rate of $2000 \mathrm{~Hz}$ ); and

- a Crossbow NAV420C A-100 GPS-aided MEMS inertial system (sampling rate of $100 \mathrm{~Hz})$.

The Nav420 inertial system provided acceleration and angular orientation data. Fluctuations were found in the time increments at which accelerations were recorded by the CXL accelerometers. For this reason, it was not possible to synchronize the orientation data of the NAV420 with the acceleration data of the CXL accelerometers. As a result, only the Nav420 data was considered in the analysis described in the remainder of this chapter. Furthermore, some data was missing from the first day of sea trial; therefore the last four days were considered in the analysis (June $12^{\text {th }}-15^{\text {th }}$, 2007).

The processing of the data involved three operations: accelerometer gravity offset removal, filtering, and impact pulse extraction and organisation. 

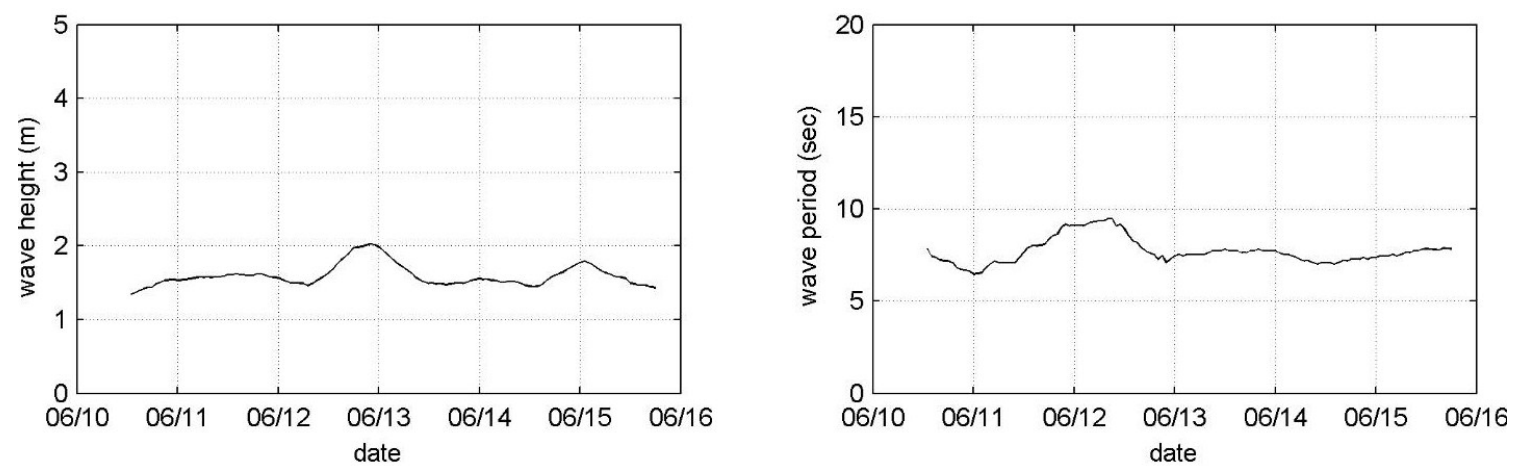

Figure 2.1: Wave height and period [provided by DRDC].

\subsubsection{Accelerometer Gravity Offset}

A tri-axial accelerometer measures specific force [13]:

$$
\vec{f}=\vec{a}-\vec{g}
$$

where $\vec{f}$ is the specific force, $\vec{a}$ is the inertial acceleration, and $\vec{g}$ is the gravitational vector. Therefore, an accelerometer at rest will indicate $-\vec{g}$. This gravity offset was removed from the data by finding the components of $\vec{g}$ in the local frame of the boat and then adding these components to the accelerometer outputs. The $x y z$ Euler angle sequence was used to determine $\vec{g}_{\text {local }}$ :

$$
\vec{g}_{\text {local }}=-9.81\left(\begin{array}{c}
\sin \theta_{x} \sin \theta_{z}-\cos \theta_{x} \sin \theta_{y} \cos \theta_{z} \\
\sin \theta_{x} \cos \theta_{z}+\cos \theta_{x} \sin \theta_{y} \sin \theta_{z} \\
\cos \theta_{x} \cos \theta_{y}
\end{array}\right)
$$


where $\theta_{x}$ is the roll angle, $\theta_{y}$ is the pitch angle, and $\theta_{z}$ the yaw angle in a righthanded coordinate system where the $\mathrm{x}$-axis points towards the bow of the boat and the z-axis points towards the sky.

\subsubsection{Filtering}

Generally, the frequency range of rigid body motion of a HSC does not exceed $10 \mathrm{~Hz}$ (Figure 2.2). A $10 \mathrm{~Hz}$ low-pass fourth-order Butterworth filter was applied to the data in order to eliminate high-frequency acceleration spikes resulting from structural vibration of the boat deck. Furthermore, a $0.05 \mathrm{~Hz}$ high-pass fourth-order Butterworth filter was applied in order to remove any offset caused by accelerometer bias.

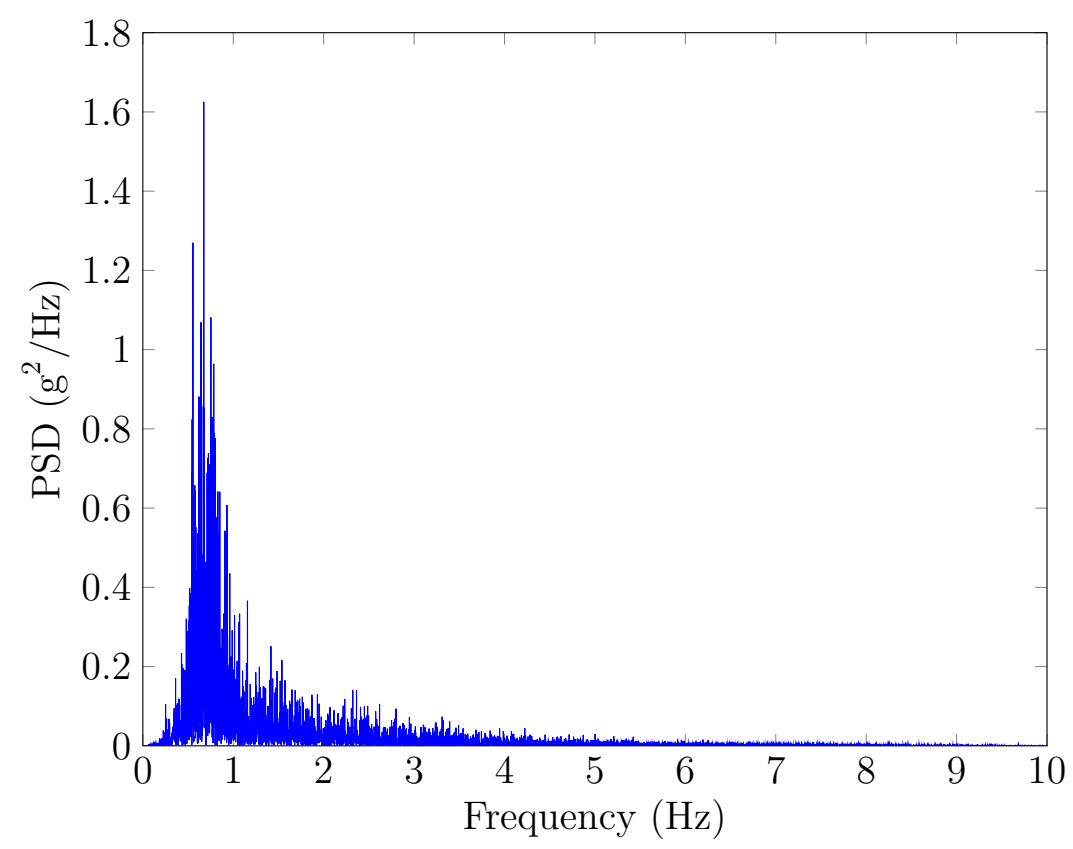

Figure 2.2: Power spectral density of unfiltered vertical acceleration of the midships portion of a RHIB. 


\subsubsection{Impact Pulse Extraction}

There is no universally-accepted value defining the maximum acceleration of a slam event. Peak values observed in the data were up to $3.8 \mathrm{~g}$ (Figure 2.3). For the analysis presented in this thesis, an acceleration of $2 \mathrm{~g}$ was chosen as the minimum acceleration peak for an impact event. $2 \mathrm{~g}$ is approximately the median of the acceleration values. An algorithm was written to extract impact pulses based on the $2 \mathrm{~g}$ threshold. This threshold, however, can be changed in the developed algorithm and corresponding computer implementation, to a different value if a more precise threshold is determined as a result of future advancements into HSC impact motion research. The pulses were then normalized such that their peak values and durations were both equal to one. Linear interpolation was used at the extremities such that the curves pass through $(0,0)$ and $(1,0)$ (Figure 2.4).

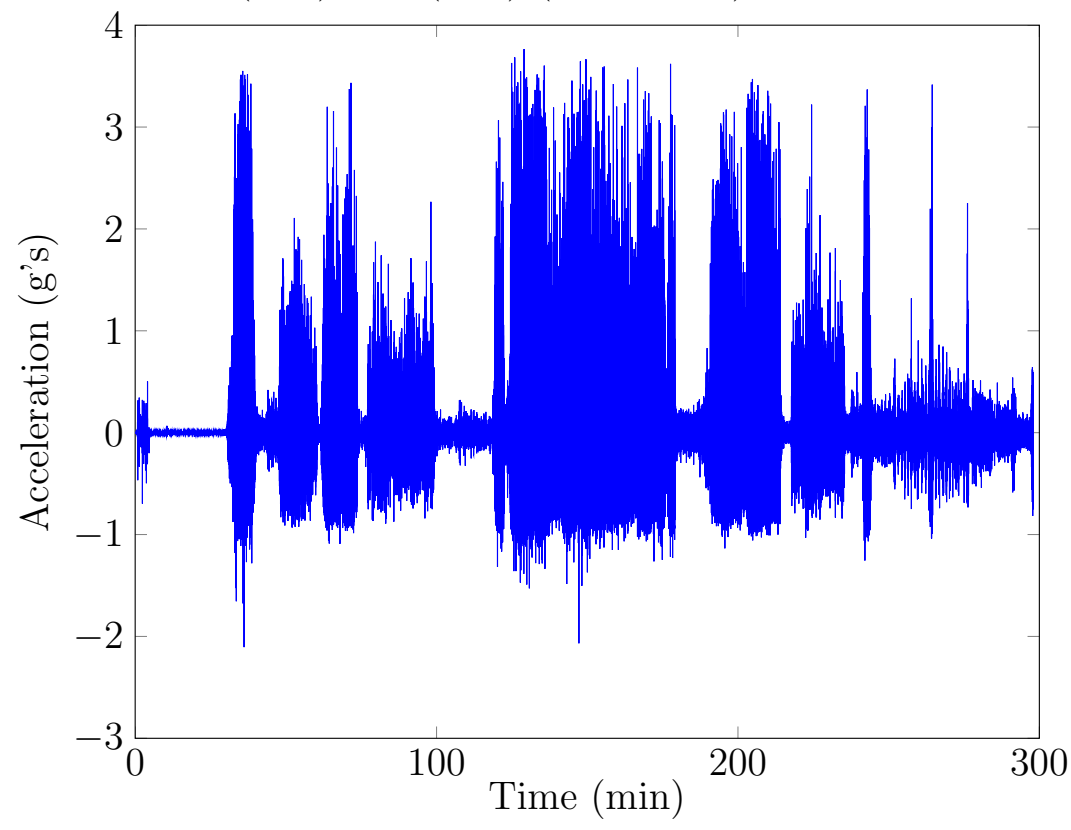

Figure 2.3: Vertical acceleration time history of the midships portion of a RHIB. 


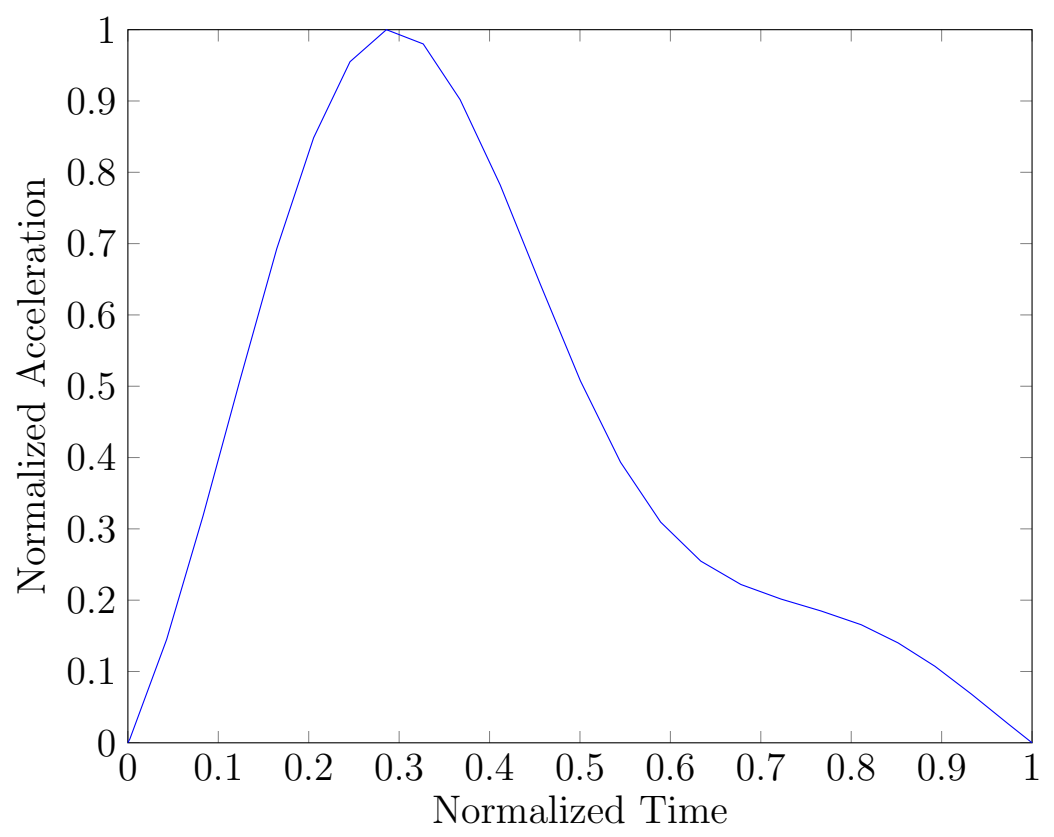

Figure 2.4: Typical normalized acceleration pulse.

\subsection{Acceleration Profiles}

After a thorough inspection of the acceleration curves, five general types of pulses were identified. The equations which were used to curve fit the data consist of a combination of modified Gaussian terms:

$$
F(x)=\sum_{i=1}^{n} x(x-1) a_{i} \mathrm{e}^{-\left(\frac{x-m_{i}}{\sigma_{i}}\right)^{2}}
$$

where $x$ is normalized time and $F(x)$ is normalized acceleration; also associated with each term is a scaling parameter $a_{i}$, a location parameter $m_{i}$ and a shape parameter 
$\sigma_{i}$. Initially a different equation form was used:

$$
F(x)=\left(1+k \sin \left(\frac{\pi x}{c+\omega x}\right)^{j}\left(\mathrm{e}^{-z x}+a\right)\right) \sum_{i=1}^{n} A_{i} \sin (\pi x) \mathrm{e}^{-u_{i} x} \text { where } j=1,2 \text { or } 3
$$

which is the product of a modified decaying sinusoid and a sum of modified half-sine curves.

While Equation 2.4 was found to be suitable for fitting the pulses, it was not well suited for time history generation (Chapter 3). The reason for this is that the parameters of Equation 2.4 are highly correlated. Efforts to rewrite and rearrange the equation were made so as to "de-correlate" the parameters were not successful.

The number of terms in Equation 2.3 varies from 1 to 5 depending on the type of pulse and its characteristics. In the following sections, the different types are presented. In all subsequent derivations $x$ is normalized time and $F(x)$ is normalized acceleration.

\subsubsection{Type 1: Half-Sine}

Shown in Figure 2.5 is a Type 1 profile. This curve resembles a half-sine which can be represented by:

$$
F(x)=x(x-1) a_{1} \mathrm{e}^{-\left(\frac{x-m_{1}}{\sigma_{1}}\right)^{2}}
$$

The location parameter $m_{1}$ can be written in terms of the peak time $x_{p}$ as follows:

$$
\begin{aligned}
\frac{\mathrm{d} F\left(x_{p}\right)}{\mathrm{d} x} & =\left(\left(2 x_{p}-1\right)-\frac{2}{\sigma_{1}^{2}} x_{p}\left(x_{p}-m_{1}\right)\right) a_{1} \mathrm{e}^{-\left(\frac{x-m_{1}}{\sigma_{1}}\right)^{2}}=0 \\
\Rightarrow m_{1} & =\frac{-\left(2 x_{p}-1\right) \sigma_{1}^{2}}{2 x_{p}\left(x_{p}-1\right)}+x_{p}
\end{aligned}
$$


Furthermore, $a_{1}$ is written as follows:

$$
\begin{aligned}
& F\left(x_{p}\right)=x_{p}\left(x_{p}-1\right) a_{1} \mathrm{e}^{-\left(\frac{x_{p}-m_{1}}{\sigma_{1}}\right)^{2}}=1 \\
& \Rightarrow a_{1}=\frac{1}{x_{p}\left(x_{p}-1\right) \mathrm{e}^{-\left(\frac{x_{p}-m_{1}}{\sigma_{1}}\right)^{2}}}
\end{aligned}
$$

The final equation is then:

$$
F(x)=\frac{x(x-1) \mathrm{e}^{-\left(\frac{x-m_{1}}{\sigma_{1}}\right)^{2}}}{x_{p}\left(x_{p}-1\right) \mathrm{e}^{-\left(\frac{x_{p}-m_{1}}{\sigma_{1}}\right)^{2}}}
$$

The equation parameters are the peak time $x_{p}$ and shape parameter $\sigma_{1}$ which controls the sharpness of the pulse. Due to the fact that the data was sampled at a relatively low rate $(100 \mathrm{~Hz})$, the peak time obtained from a given curve might be slightly offset from the peak time of a perfectly-smooth version of this curve. For this reason $x_{p}$ was treated as a variable in the curve fitting process. However, $x_{p}$ was constrained to be close to the observed peak time. This constraint was applied by setting appropriate lower and upper bounds for $x_{p}$ in the built-in Matlab function presented in Section 2.3. The specific values of the upper and lower bounds for each parameter are included in the code provided in Appendix C. 


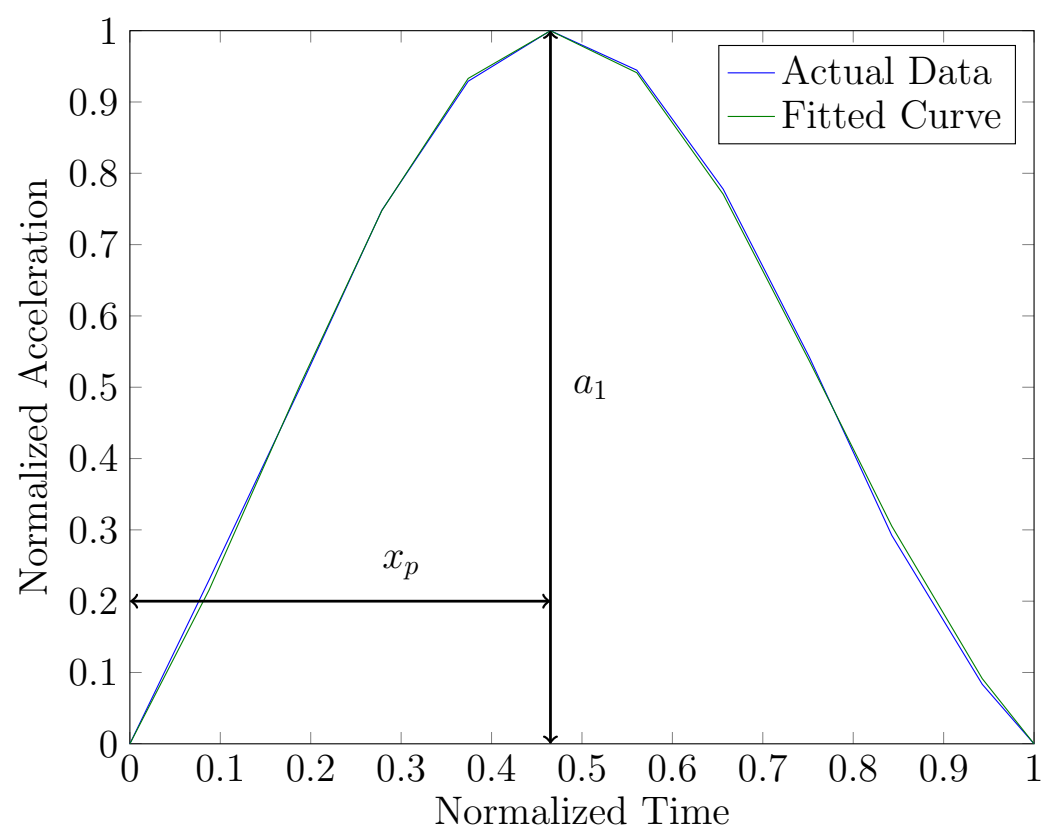

Figure 2.5: Type 1 profile.

\subsubsection{Type 2: Half-Sine with Single Shoulder}

Shown in Figure 2.6 is a Type 2 profile. This pulse consists of a half-sine followed by a shoulder or bump. The equation for this pulse is:

$$
F(x)=\frac{x(x-1) a_{1} \mathrm{e}^{-\left(\frac{x-m_{1}}{\sigma_{1}}\right)^{2}}}{x_{1}\left(x_{1}-1\right) \mathrm{e}^{-\left(\frac{x_{1}-m_{1}}{\sigma_{1}}\right)^{2}}}+\frac{x(x-1) a_{2} \mathrm{e}^{-\left(\frac{x-m_{2}}{\sigma_{2}}\right)^{2}}}{x_{2}\left(x_{2}-1\right) \mathrm{e}^{-\left(\frac{x_{2}-m_{1}}{\sigma_{1}}\right)^{2}}}
$$

where $m_{1}$ and $m_{2}$ are functions of $x_{1}$ and $x_{2}$ respectively:

$$
m_{1}=\frac{-\left(2 x_{1}-1\right) \sigma_{1}^{2}}{2 x_{1}\left(x_{1}-1\right)}+x_{1}, \quad m_{2}=\frac{-\left(2 x_{2}-1\right) \sigma_{2}^{2}}{2 x_{2}\left(x_{2}-1\right)}+x_{2}
$$


and $a_{1}$ and $a_{2}$ are the peak values of the first and second terms respectively. The expressions for $m_{1}$ and $m_{2}$ were obtained in the same fashion as for Type 1 (Equation 2.6). In addition, a shape parameter $\sigma$ is associated with each term. Parameter $a_{1}$ was constrained to be equal to approximately 1 ; as a result, it can be assumed that $x_{1}$ coincides with $x_{p}$. Using this assumption it is possible to express $a_{2}$ in terms of $h_{2}$ which is the value of the curve at the peak time of the second term:

$$
\begin{gathered}
F\left(x_{2}\right)=\frac{x_{2}\left(x_{2}-1\right) a_{1} \mathrm{e}^{-\left(\frac{x_{2}-m_{1}}{\sigma_{1}}\right)^{2}}}{x_{1}\left(x_{1}-1\right) \mathrm{e}^{-\left(\frac{x_{1}-m_{1}}{\sigma_{1}}\right)^{2}}+a_{2}}=h_{2} \\
a_{2}=h_{2}-\frac{x_{2}\left(x_{2}-1\right) a_{1} \mathrm{e}^{-\left(\frac{x_{2}-m_{1}}{\sigma_{1}}\right)^{2}}}{x_{1}\left(x_{1}-1\right) \mathrm{e}^{-\left(\frac{x_{1}-m_{1}}{\sigma_{1}}\right)^{2}}}
\end{gathered}
$$

However, for the curve fitting process, the variable $a_{2}$ was used instead of $h_{2}$. The reason for this is that $h_{2}$ is close to but not necessarily equal to the amplitude of a known characteristic point on the curve such as an inflection point or a local minimum. Parameter $a_{2}$ was therefore used in order to reduce computational time. 


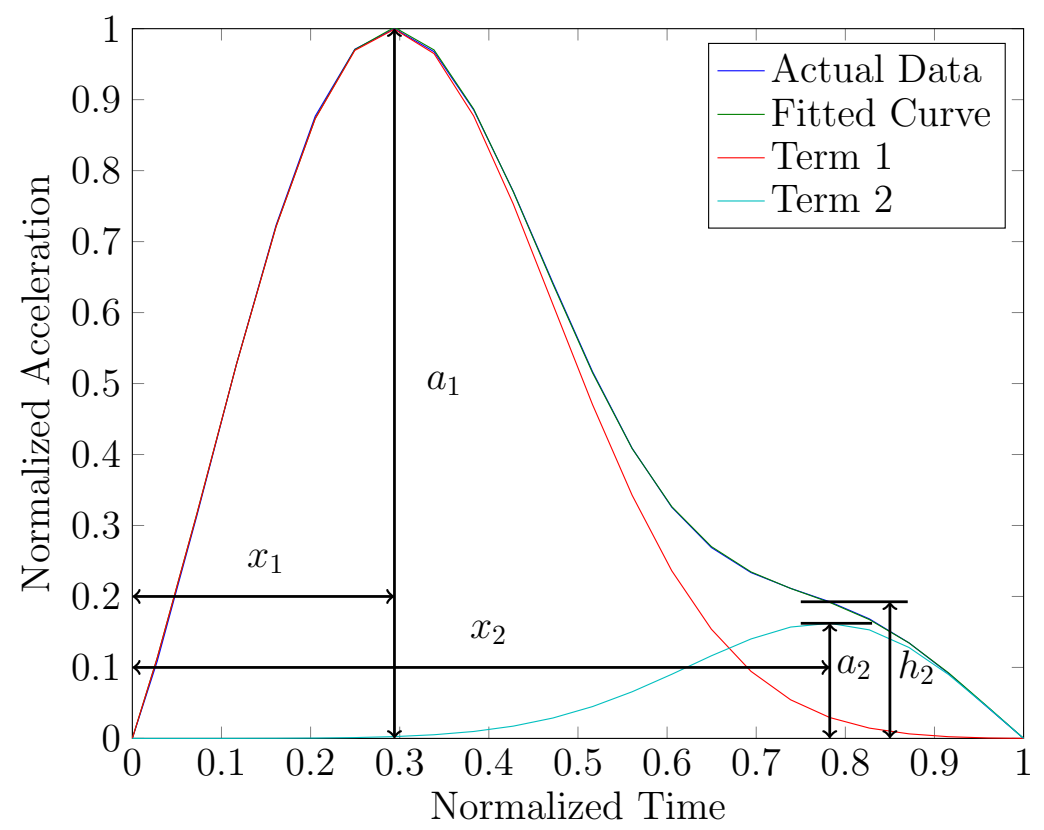

Figure 2.6: Type 2 profile.

\subsubsection{Type 3: Half-Sine with Multiple Shoulders}

Type 3 profiles are characterized by a half-sine shape followed by 2, 3, or 4 shoulders as shown in Figures 2.7 through 2.9, respectively. Type 3 pulses were classified according to the number of shoulders following the main peak:

- Type 3.I: half-sine followed by 2 shoulders;

- Type 3.II: half-sine followed by 3 shoulders; and

- Type 3.III: half-sine followed by 4 shoulders.

Three equations are therefore necessary:

$$
F_{i}(x)=\sum_{j=1}^{i+1} a_{j} \frac{x(x-1) \mathrm{e}^{-\left(\frac{x-m_{j}}{\sigma_{j}}\right)^{2}}}{x_{j}\left(x_{j}-1\right) \mathrm{e}^{-\left(\frac{x_{j}-m_{j}}{\sigma_{j}}\right)^{2}}} \quad i=2,3, \text { and } 4
$$




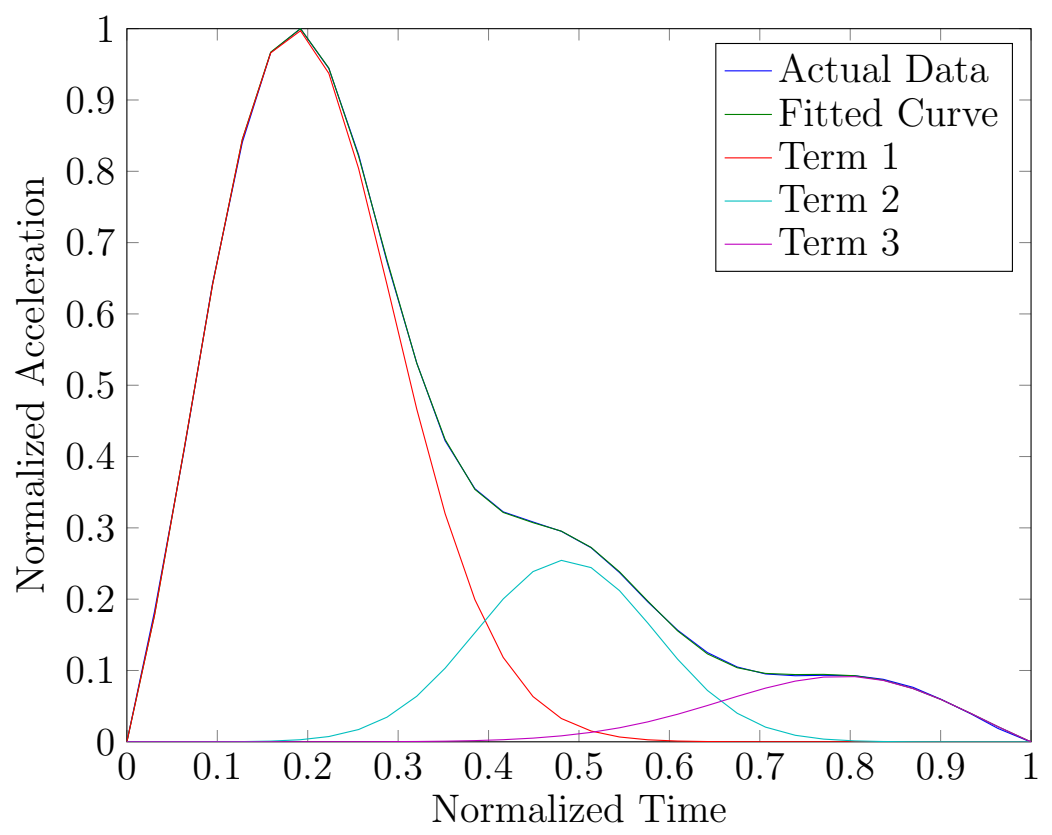

Figure 2.7: Type 3.I profile.

where the index $i$ is the number of shoulders. The equation parameters are treated similarly as those of Types 1 and 2:

$$
m_{i}=\frac{-\left(2 x_{i}-1\right) \sigma_{i}{ }^{2}}{2 x_{i}\left(x_{i}-1\right)}+x_{i}
$$




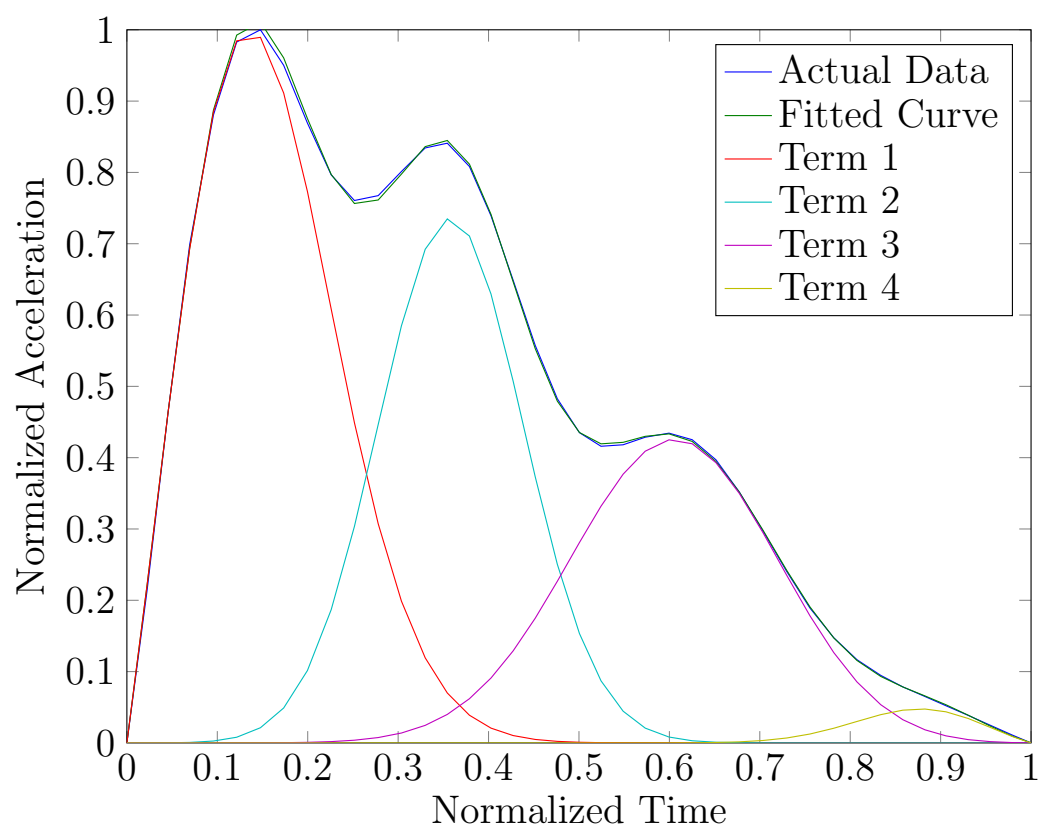

Figure 2.8: Type 3.II profile.

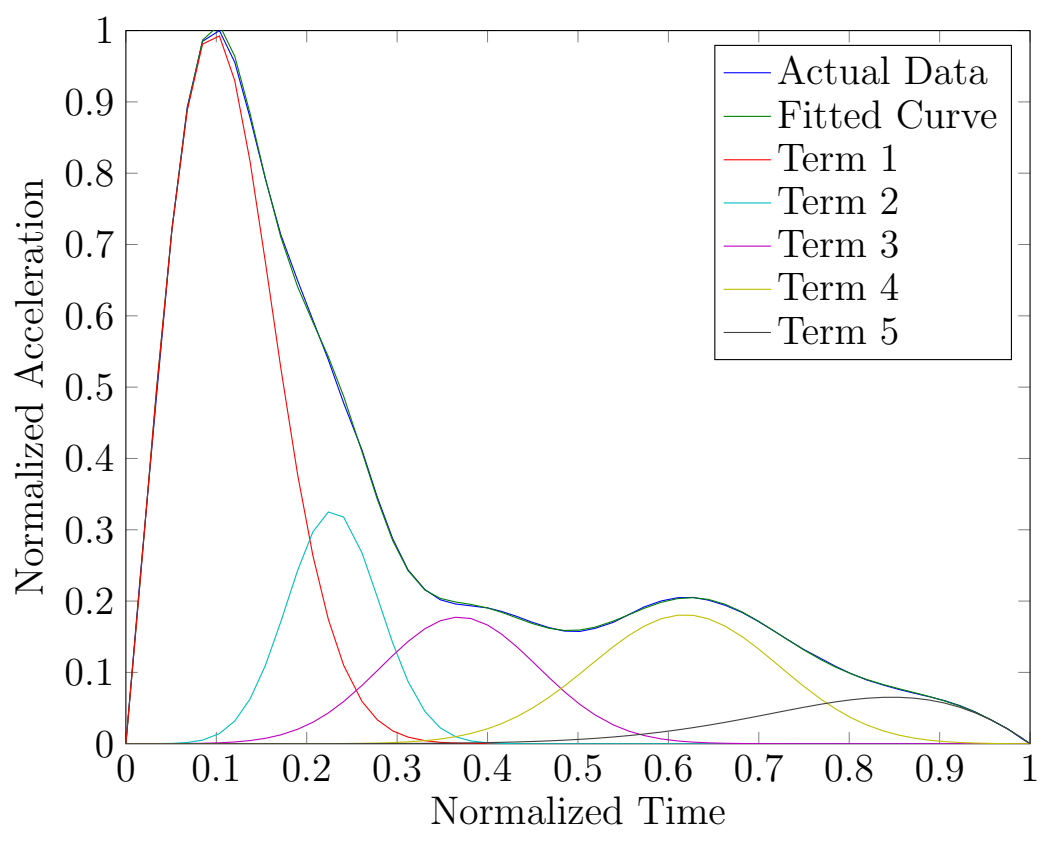

Figure 2.9: Type 3.III profile. 


\subsubsection{Type 4: Delayed Peak}

In Type 4 profiles the peak time is generally more delayed and the main peak is preceded by a shoulder as shown in Figures 2.10 through 2.12. Similarly to Type 3, Type 4 includes three variations, each one with a particular number of underlying curves:

- Type 4.I: 1 shoulder, followed by main peak, followed by 1 shoulder;

- Type 4.II: 1 shoulder, followed by main peak, followed by 2 shoulders; and

- Type 4.III: 1 shoulder, followed by main peak, followed by 3 shoulders.

The representative equation is the same as for Type 3:

$$
F_{i}(x)=\sum_{j=1}^{i+1} a_{j} \frac{x(x-1) \mathrm{e}^{-\left(\frac{x-m_{j}}{\sigma_{j}}\right)^{2}}}{x_{j}\left(x_{j}-1\right) \mathrm{e}^{-\left(\frac{x_{j}-m_{j}}{\sigma_{j}}\right)^{2}}}
$$

where $i$ is the number of shoulders and, as opposed to the previous types, $x_{2}<x_{1}$. Similarly to the previous types:

$$
m_{i}=\frac{-\left(2 x_{i}-1\right) \sigma_{i}^{2}}{2 x_{i}\left(x_{i}-1\right)}+x_{i}
$$




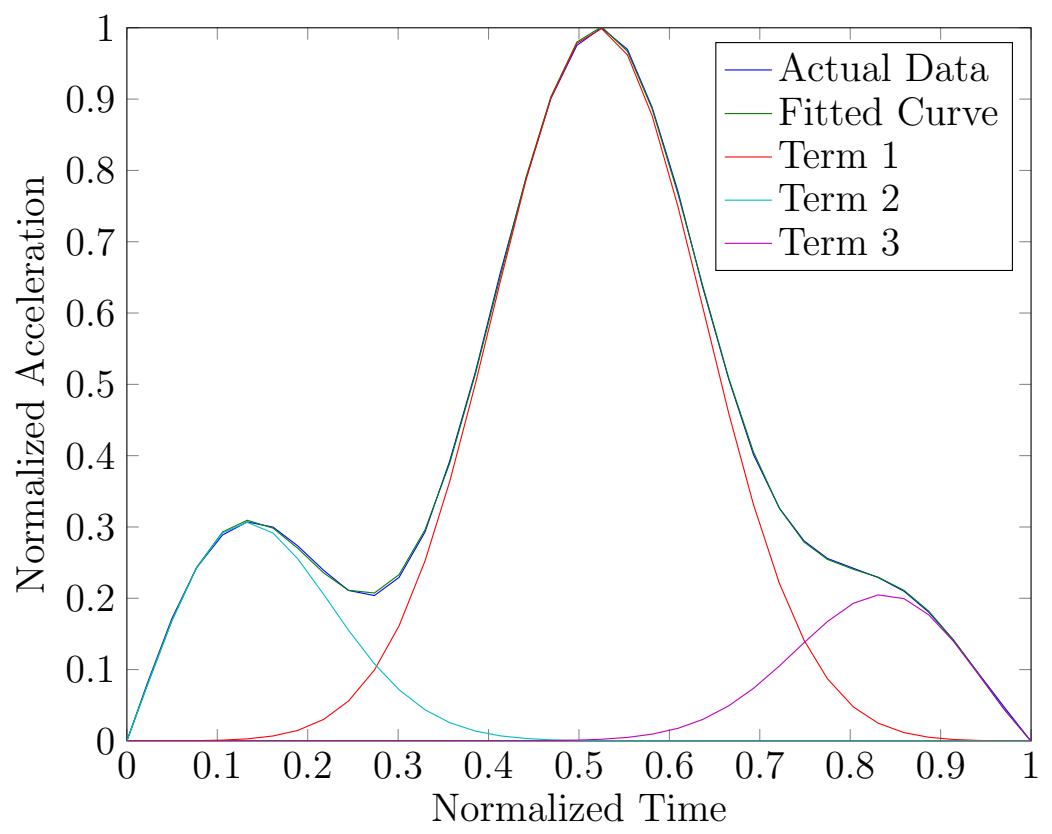

Figure 2.10: Type 4.I profile.

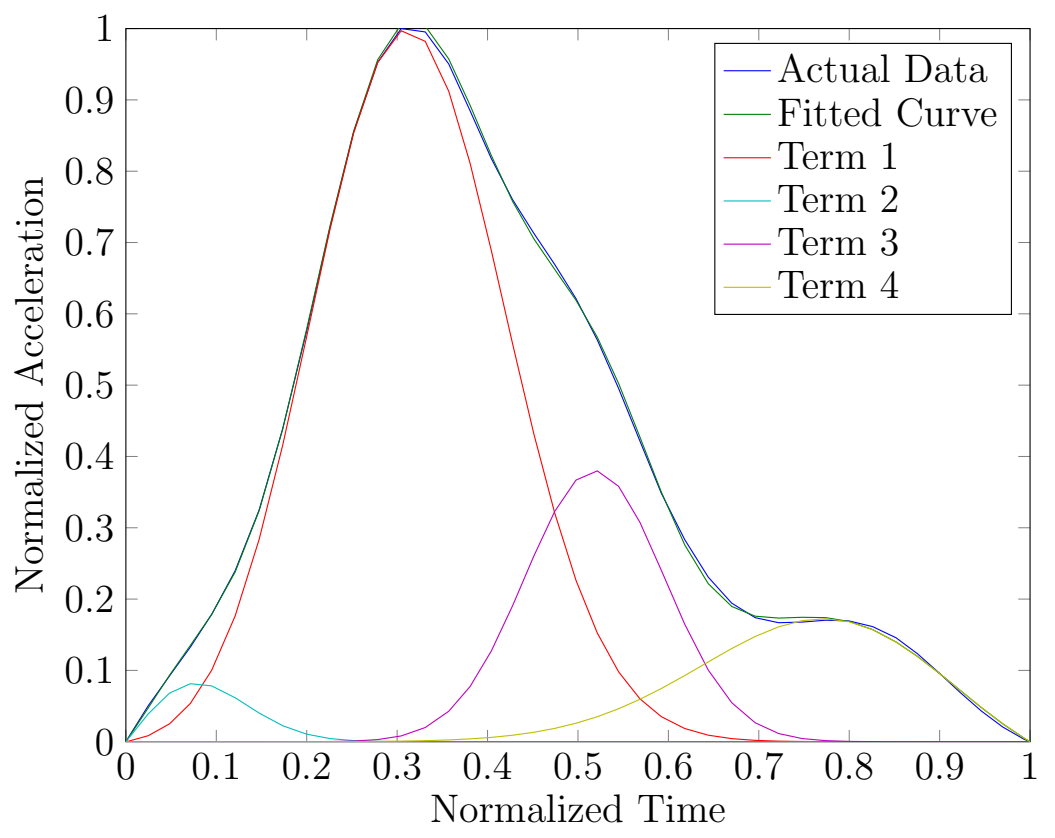

Figure 2.11: Type 4.II profile. 


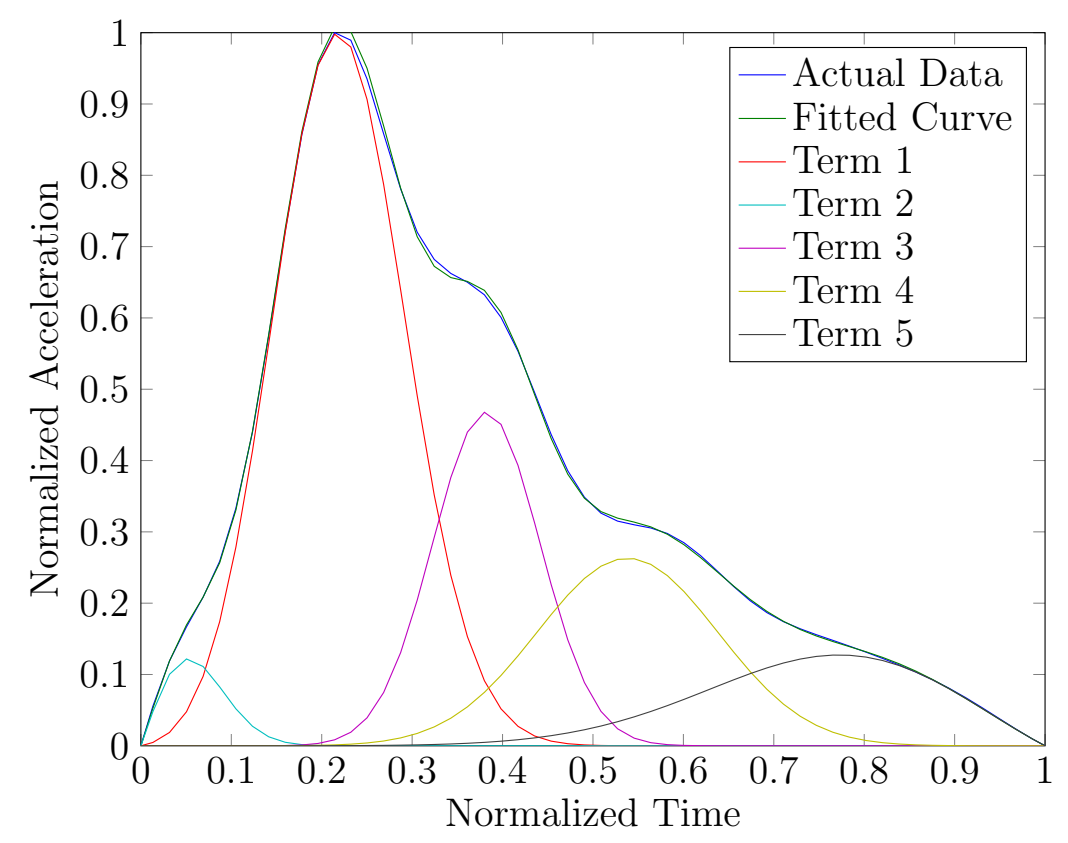

Figure 2.12: Type 4.III profile.

\subsubsection{Type 5: Double peak}

Type 5 profiles consist of two successive impacts. In Type 5 profiles the acceleration does not die out after the first peak but picks up again as shown in Figures 2.13 through 2.15. The equation for Type 5 profiles is:

$$
F_{i}(x)=\sum_{j=1}^{i} a_{j} \frac{x(x-1) \mathrm{e}^{-\left(\frac{x-m_{j}}{\sigma_{j}}\right)^{2}}}{x_{j}\left(x_{j}-1\right) \mathrm{e}^{-\left(\frac{x_{j}-m_{j}}{\sigma_{j}}\right)^{2}}}
$$

where $i$ can vary from 3 to 5 depending on how many parameters are neccessary to model a particular Type 5 pulse.

A number of different variations can occur in this type of profile. The following numbering convention has been chosen in order to refer to the different variations: 
Table 2.1: Observed Type 5 variations.

\begin{tabular}{|c|c|}
\hline Number of Gaussian Terms & Observed Variations \\
\hline 3 & $1-2,2-1,1-2^{r}$ \\
\hline 4 & 1 1-3.I, 1-4.I, 2-2, 3.I-1, 4.I-1, 1-3.I ${ }^{r}, 2-2^{r}$ \\
\hline 5 & 1-3.II, 1-4.II, 2-3.I, 2-4.I, 3.I-2, 1-3.II ${ }^{r}, 1-4 . \mathrm{II}^{r}$ \\
\hline
\end{tabular}

- a double peak profile consisting of a Type $\mathrm{x}$ followed by a Type $\mathrm{y}$, where the maximum amplitude is reached during the first profile, is denoted as Type $\mathrm{x}-\mathrm{y}$; and - a double peak profile consisting of a Type $\mathrm{x}$ followed by a Type $\mathrm{y}$, where the maximum amplitude is reached during the second profile, is denoted as Type $\mathrm{x}-\mathrm{y}^{r}$ (where $r$ stands for reverse). For example, a Type 3.I followed by a Type 2, where the maximum amplitude is reached during the second profile, is denoted as Type 3.I- $2^{r}$. The observed variations are outlined in Table 2.1.

To detect Type 5 pulses the minimum point between the two peaks $\left(x_{\min }, a_{\min }\right)$, shown in Figure 2.13, is first identified. If the difference in amplitude between this minimum point and the second highest peak $(\Delta a)$ is larger than a certain threshold then the pulse is considered of Type 5. A threshold of 0.2 was determined for $\Delta a$ based on the inspection of double peak pulses. Curve fitting this type of pulse as a whole is an alternative to considering the two peaks as separate events and fitting each one individually. 


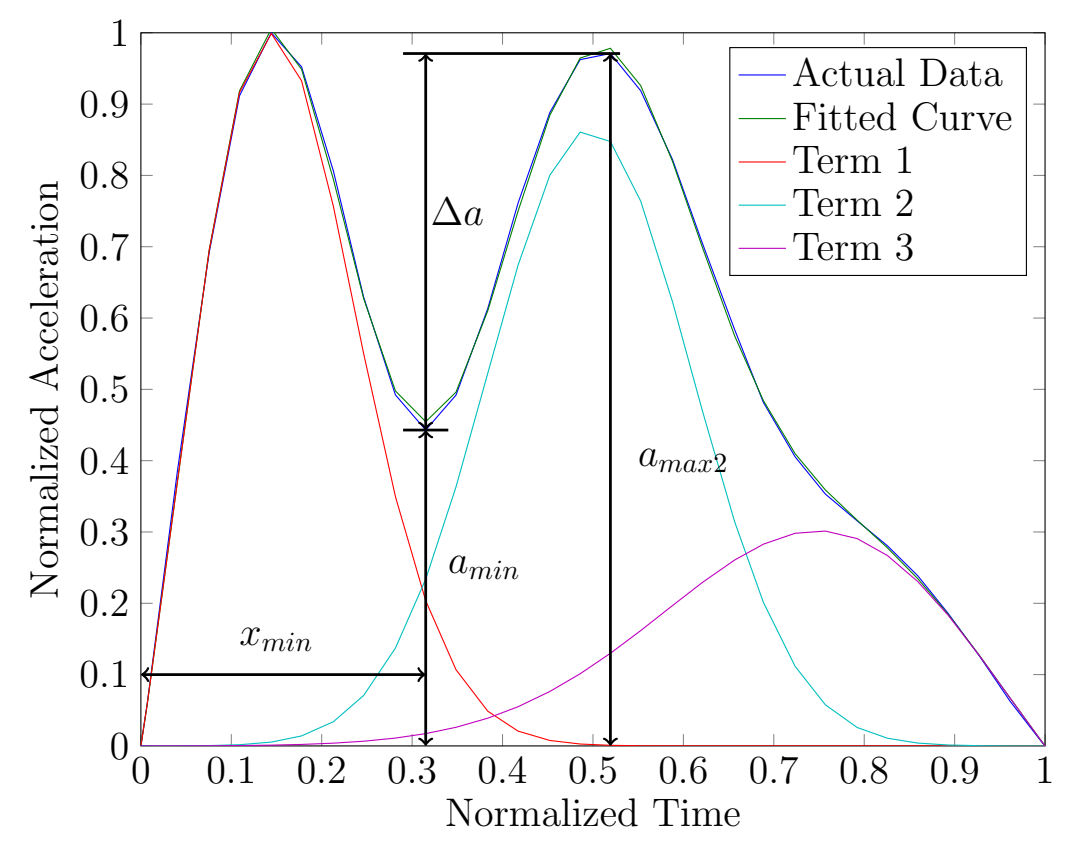

Figure 2.13: Type 1-2 profile.

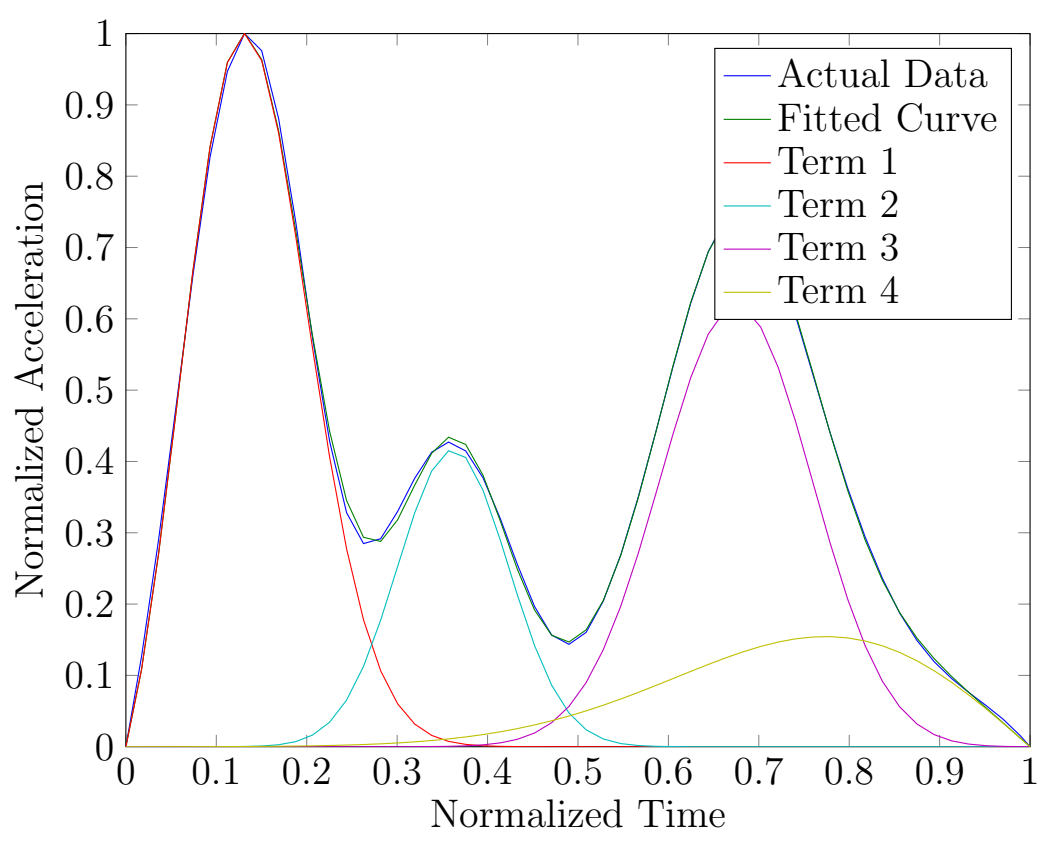

Figure 2.14: Type 2-2 profile. 


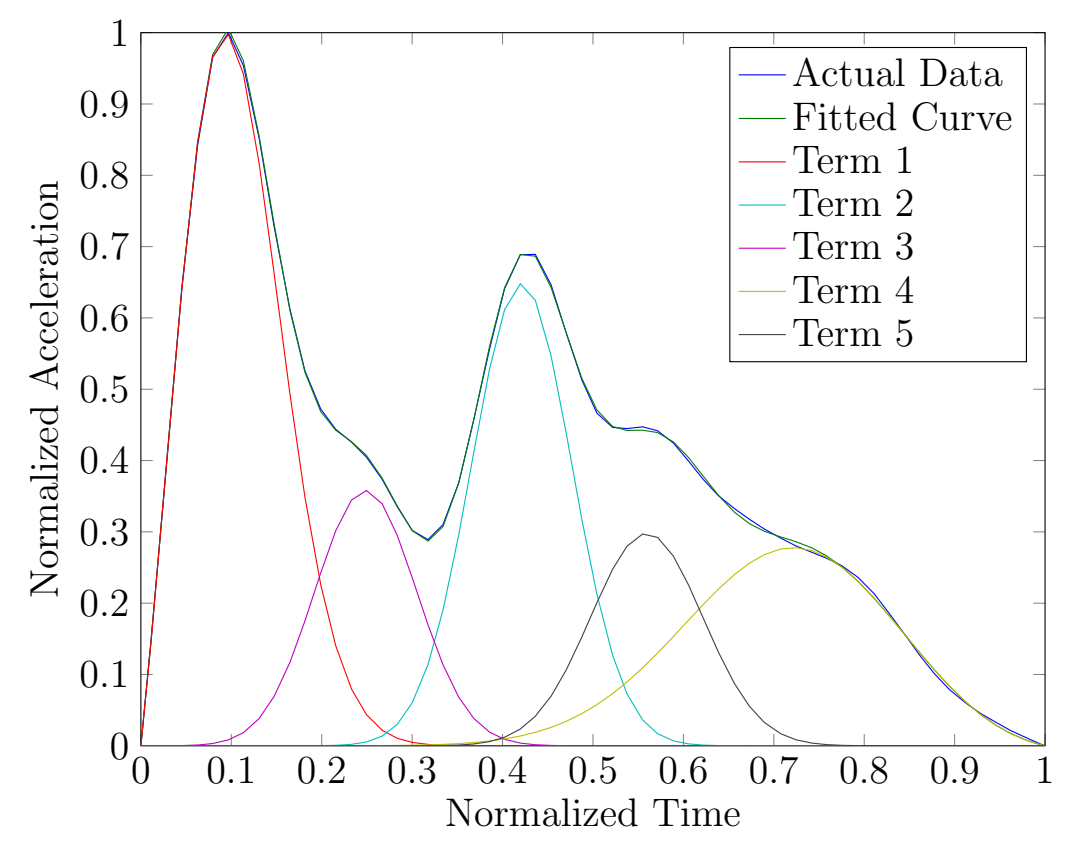

Figure 2.15: Type 2-3.I profile.

\subsection{Curve Fitting Algorithm}

A built-in Matlab function (lsqcurvefit) was used for curve fitting the acceleration pulses. Initial guesses of the parameter values are input to the function as well as parameter constraints (upper and lower bounds). The output is a set of parameter values which minimizes the sum of the squares of the residuals (SR) between the original data and the fitted curve. The algorithm is sensitive to initial guesses and constraints; for this reason different starting points and bounds were used in curve fitting the data. A maximum SR below which a fitted curve is considered acceptable was set based on experience. The threshold SR is not however a fixed value but is proportional to the number of data points and to an average residual at each data 
point:

$$
\mathrm{SR}_{\text {threshold }}=(\text { Average Residual Threshold })^{2} \times \text { number of data points }
$$

The average residual threshold was determined as 0.01 after a thorough inspection of the fitted curves.

\subsubsection{Pulse Type Detection}

A two-step procedure was followed in order to detect the different types of profiles from the observed pulses automatically. For each pulse, the first step was to determine the number of equation terms which are necessary to obtain a matching fitted curve according to the established $\mathrm{SR}_{\text {threshold }}$. This was performed by successive curve fitting with increasing number of terms until the obtained SR was lower than the $\mathrm{SR}_{\text {threshold}}$. Sequential fitting was used in order to obtain the best possible fit with the least number of equation parameters/terms. The second step was to classify the detected pulse, from the first step, into the different types. For Types 1 and 2, the second step was not necessary since these profiles are not characterized by any variations. The remaining types were detected by a two-step elimination process. First it is determined whether or not the pulse is a Type 5 by the procedure described in Section 2.2.5. Second, if the pulse is not a Type 5, then it is a Type 4 if one of the underlying curves has a peak time smaller than the peak time of the overall pulse. If the previous criteria are not met then a Type 3 is identified. It was observed that in some cases, the second highest peak of a Type 5 profile precedes the highest peak. For this reason, Type 5 takes precedence over Type 4 in the elimination process. 


\subsection{Reduction of Sea Trial Data}

Four pairs of time histories that were collected (by DRDC) over four consecutive days were analyzed. Each pair consists of the time histories of the forward portion of the boat and the centre portion as mentioned in Section 2.1. In Table 2.2, the pulse detection rate for each time history is shown. In Figures 2.16 through 2.23, the profile distribution for each time history is presented. The parameter mean values for each type are shown in Tables 2.3 through 2.10. The last two columns in each table correspond to the mean peak acceleration and pulse duration, respectively. Types 1 through 4 can be visualized in Figure 2.24.

An additional data set, also provided by DRDC, has been reduced. The results are presented in Appendix A.

Table 2.2: Pulse detection rate.

\begin{tabular}{|l|l|l|l|}
\hline Day & Position on Boat & Number of Pulses & Detection Rate (\%) \\
\hline \multirow{2}{*}{1} & Midships & 17 & 100 \\
\cline { 2 - 4 } & Bow & 712 & 92 \\
\hline \multirow{2}{*}{2} & Midships & 232 & 86 \\
\cline { 2 - 4 } & Bow & 1949 & 92 \\
\hline \multirow{2}{*}{3} & Midships & 46 & 83 \\
\cline { 2 - 4 } & Bow & 1196 & 93 \\
\hline \multirow{2}{*}{4} & Midships & 192 & 88 \\
\cline { 2 - 4 } & Bow & 2305 & 90 \\
\hline
\end{tabular}




\begin{tabular}{|c|c|c|c|c|c|c|c|c|}
\hline 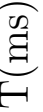 & $\stackrel{m}{\infty}$ & $\stackrel{\infty}{\stackrel{\infty}{\sim}}$ & $\stackrel{0}{\not}$ & $\begin{array}{l}\stackrel{0}{6} \\
0\end{array}$ & $\stackrel{\stackrel{2}{N}}{\stackrel{N}{N}}$ & i⿱ & iิ & 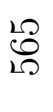 \\
\hline $\begin{array}{l}\frac{\pi}{20} \\
\frac{50}{\pi}\end{array}$ & $\stackrel{+!}{\sim}$ & $\stackrel{\llcorner\rho}{\sim}$ & $\stackrel{\sim}{\sim}$ & $\stackrel{\kappa}{\sim}$ & $\begin{array}{l}0 \\
\text { i }\end{array}$ & $\vec{v}$ & $\stackrel{\leftrightarrow}{\sim}$ & $\stackrel{\forall}{\Delta}$ \\
\hline 6 & & & & & $\begin{array}{l}\stackrel{\overbrace{}}{0} \\
0\end{array}$ & & & 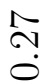 \\
\hline$\stackrel{20}{\pi}$ & & & & & $\stackrel{N}{1}$ & & & $\begin{array}{l}0 \\
0 \\
0\end{array}$ \\
\hline 8 & & & & & 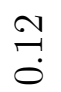 & & & $\stackrel{\vec{H}}{\overrightarrow{0}}$ \\
\hline$b^{+}$ & & & & $\stackrel{\leftrightarrow}{3}$ & $\stackrel{\overbrace{}}{0}$ & & $\stackrel{100}{\stackrel{0}{0}}$ & $\stackrel{m}{\stackrel{\infty}{0}}$ \\
\hline त् & & & & $\begin{array}{l}\mathscr{0} \\
0 \\
0\end{array}$ & $\begin{array}{l}\text { i⿱⺈ } \\
\text { î } \\
0\end{array}$ & & $\tilde{E}$ & $\stackrel{10}{20}$ \\
\hline है & & & & $\stackrel{\vec{H}}{\overrightarrow{0}}$ & $\stackrel{\leftrightarrow}{\overrightarrow{0}}$ & & $\begin{array}{l}0 \\
\stackrel{1}{0}\end{array}$ & $\stackrel{20}{\stackrel{10}{0}}$ \\
\hline$b^{\infty}$ & & & 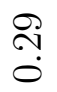 & $\stackrel{0}{\stackrel{0}{0}}$ & $\vec{b}$ & $\stackrel{0}{\stackrel{0}{0}}$ & $\stackrel{\stackrel{\sim}{Z}}{\circ}$ & $\stackrel{\infty}{\stackrel{0}{0}}$ \\
\hline $\mathbb{Z}^{\infty}$ & & & $\begin{array}{l}0 \\
\mathscr{0} \\
0\end{array}$ & $\stackrel{\not}{\not}$ & $\stackrel{\mathscr{L}}{\mathscr{2}}$ & $\stackrel{+}{0}$ & $\begin{array}{l}0 \\
0 \\
0\end{array}$ & $\stackrel{10}{+}$ \\
\hline$\ddot{8}$ & & & $\stackrel{\vec{H}}{\stackrel{4}{0}}$ & $\stackrel{\overbrace ָ}{ٍ}$ & 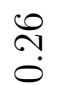 & 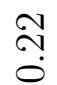 & $\stackrel{\vec{N}}{\stackrel{H}{0}}$ & 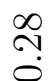 \\
\hline$b^{N}$ & & $\stackrel{\vec{N}}{0}$ & $\stackrel{\infty}{\stackrel{\infty}{0}}$ & $\stackrel{\infty}{\stackrel{\infty}{0}}$ & $\begin{array}{l}\mathscr{Q} \\
\stackrel{0}{0}\end{array}$ & $\stackrel{\sim}{\stackrel{\sim}{0}}$ & $\stackrel{\circ}{\stackrel{0}{0}}$ & $\ddot{\rightleftarrows}$ \\
\hline ॄे & & O̊ & $\underset{+}{\not}$ & 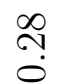 & 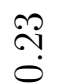 & $\underset{ }{\not}$ & $\stackrel{g}{\stackrel{0}{0}}$ & $\stackrel{\infty}{\stackrel{0}{0}}$ \\
\hline $\mathcal{B}$ & & $\stackrel{\infty}{\overrightarrow{0}}$ & 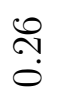 & $\stackrel{\overbrace{}}{\stackrel{O}{0}}$ & $\stackrel{\leftrightarrow}{\leftrightarrow}$ & $\stackrel{0}{\rightleftarrows}$ & $\stackrel{\overbrace{}}{\stackrel{\overbrace{}}{0}}$ & $\stackrel{\overbrace ָ}{\stackrel{0}{0}}$ \\
\hline 5 & $\begin{array}{l}\text { ip } \\
\text { i? }\end{array}$ & $\begin{array}{l}\infty \\
\stackrel{\infty}{0} \\
0\end{array}$ & $\stackrel{\sim}{\stackrel{N}{0}}$ & $\stackrel{ }{\stackrel{+}{0}}$ & $\stackrel{0}{\stackrel{1}{0}}$ & 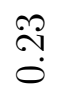 & $\begin{array}{l}0 \\
\stackrel{0}{0}\end{array}$ & $\stackrel{\sim}{\stackrel{\sim}{0}}$ \\
\hline$\vec{\pi}$ & $\stackrel{\leftrightarrow}{\sharp}$ & 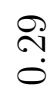 & $\stackrel{\infty}{\stackrel{\infty}{0}}$ & $\stackrel{m}{\rightleftarrows}$ & $\vec{\exists}$ & $\stackrel{n}{o}$ & مִ & $\begin{array}{l}\infty \\
\stackrel{\infty}{0} \\
\stackrel{n}{0}\end{array}$ \\
\hline 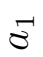 & $\neg$ & $\neg$ & - & $\neg$ & $\neg$ & - & - & $\neg$ \\
\hline & & & 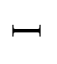 & $\boxminus$ & $\Xi$ & • & $\Xi$ & $\Xi$ \\
\hline & تِ & 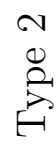 & \multicolumn{3}{|l|}{$\begin{array}{l}\infty \\
0 \\
0 \\
3 \\
0\end{array}$} & \multicolumn{3}{|l|}{$\begin{array}{l}+ \\
0 \\
3 \\
3\end{array}$} \\
\hline
\end{tabular}




\begin{tabular}{|c|c|c|c|c|c|c|c|c|c|c|c|c|c|c|c|c|c|}
\hline 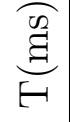 & & & $\underset{\substack{N \\
\infty}}{\mathcal{N}}$ & 昌 & $\begin{array}{l}8 \\
0 \\
0\end{array}$ & $\frac{20}{0}$ & 匇 & & 禺 & & $\stackrel{\infty}{0}$ & & 年 & 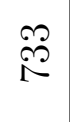 & $\overrightarrow{\mathscr{\vartheta}}$ & $\begin{array}{l}0 \\
20\end{array}$ & \\
\hline 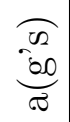 & & & $\stackrel{\vec{N}}{\mathrm{i}}$ & $\stackrel{H}{i}$ & $\underset{\sim}{\sim}$ & $\begin{array}{l}20 \\
i\end{array}$ & $\vec{i}$ & & $\stackrel{N}{\mathcal{N}}$ & & $\overrightarrow{d i}$ & & $\begin{array}{l}0 \\
\stackrel{\sim}{N}\end{array}$ & $\overrightarrow{\mathrm{i}}$ & $\begin{array}{l}0 \\
i\end{array}$ & $\stackrel{0}{\infty}$ & \\
\hline $6^{10}$ & & & & & & & & & & & 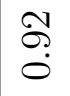 & & 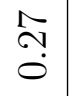 & $\stackrel{\mathfrak{7}}{\stackrel{0}{0}}$ & $\stackrel{7}{\rightleftarrows}$ & 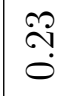 & \\
\hline 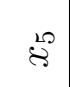 & & & & & & & & & & & $\begin{array}{l}+ \\
\infty \\
0\end{array}$ & & $\begin{array}{l}0 \\
0 \\
0\end{array}$ & $\begin{array}{l}\infty \\
1 \\
0\end{array}$ & $\mid \begin{array}{l}\infty \\
\infty \\
0\end{array}$ & $\stackrel{+}{\stackrel{1}{0}}$ & \\
\hline 8 & & & & & & & & & & & $\stackrel{20}{0}$ & & $\underset{⿱}{-}$ & $\begin{array}{l}\stackrel{\theta}{二} \\
\overrightarrow{0}\end{array}$ & $\stackrel{\sim}{\stackrel{1}{0}}$ & $\begin{array}{l}\mathscr{2} \\
0 \\
0\end{array}$ & \\
\hline$b^{+\prime}$ & & & & $\vec{m}$ & $\begin{array}{l}\vec{H} \\
\stackrel{0}{0}\end{array}$ & $\stackrel{\leftrightarrow}{\leftrightarrow}$ & $\begin{array}{l}\infty \\
\overrightarrow{0} \\
0\end{array}$ & & 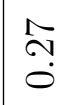 & & $\stackrel{\stackrel{2}{N}}{\stackrel{0}{0}}$ & & $\begin{array}{l}\stackrel{0}{1} \\
0\end{array}$ & $\stackrel{\sim}{\overrightarrow{7}}$ & $\begin{array}{l}\stackrel{0}{0} \\
0\end{array}$ & $\stackrel{2}{\overrightarrow{0}}$ & \\
\hline $\overrightarrow{8}$ & & & & $\begin{array}{l}R \\
0 \\
0\end{array}$ & $\begin{array}{l}0 \\
\infty \\
0 \\
0\end{array}$ & $\begin{array}{l}\Gamma \\
0\end{array}$ & $\begin{array}{l}\hat{N} \\
0\end{array}$ & & $\stackrel{\vec{r}}{\stackrel{5}{0}}$ & & $\stackrel{\text { fọ }}{0}$ & & $\begin{array}{l}\stackrel{20}{20} \\
\stackrel{2}{0} \\
0\end{array}$ & $\begin{array}{l}\vec{J} \\
0 \\
0\end{array}$ & 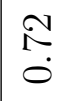 & 苞 & \\
\hline छే & & & & $\stackrel{\infty}{\rightleftarrows}$ & $\begin{array}{l}\infty \\
\stackrel{0}{0} \\
0\end{array}$ & 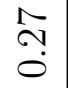 & $\begin{array}{l}0 \\
\stackrel{1}{0} \\
0\end{array}$ & & 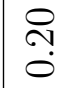 & & $\stackrel{0}{0}$ & & 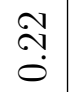 & $\begin{array}{c}\stackrel{0}{1} \\
0\end{array}$ & शి & 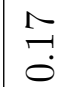 & \\
\hline$\theta^{\infty}$ & & & $\stackrel{20}{\dddot{0}}$ & $\begin{array}{l}0 \\
\stackrel{0}{0}\end{array}$ & $\stackrel{ }{\stackrel{-}{0}}$ & $\begin{array}{l}\stackrel{o}{2} \\
\stackrel{0}{0}\end{array}$ & 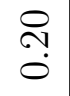 & & $\stackrel{\mathfrak{T}}{\mathfrak{0}}$ & & $\stackrel{0}{\stackrel{0}{0}}$ & & $\begin{array}{l}\infty \\
0 \\
0\end{array}$ & $\begin{array}{l}\mathscr{8} \\
0 \\
0\end{array}$ & $\stackrel{\sharp}{\rightleftarrows}$ & $\begin{array}{l}\infty \\
0 \\
0\end{array}$ & \\
\hline 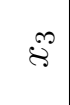 & & & $\begin{array}{l}\vec{\infty} \\
0 \\
0\end{array}$ & 年 & 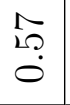 & 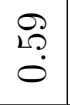 & $\vec{m}$ & & 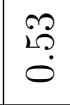 & & $\stackrel{\tilde{\sigma}}{\tilde{\sigma}}$ & & $\stackrel{\text { Iี }}{\stackrel{0}{0}}$ & $\begin{array}{l}\sqrt{50} \\
0 \\
0\end{array}$ & $\underset{0}{\sharp}$ & フี & \\
\hline$\Re$ & & & $\ddot{\infty}$ & $\stackrel{N}{\sim}$ & 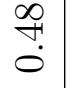 & $\begin{array}{l}20 \\
\overrightarrow{0}\end{array}$ & $\begin{array}{l}g \\
0 \\
0\end{array}$ & & ָ̃ & & ڤి & & 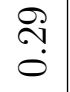 & $\begin{array}{l}\stackrel{\Re}{\sim} \\
\stackrel{0}{0}\end{array}$ & $\overrightarrow{7}$ & $\stackrel{\vec{N}}{0}$ & \\
\hline$\tilde{b}$ & & & $\stackrel{10}{\longrightarrow}$ & $\stackrel{g}{\stackrel{8}{0}}$ & $\begin{array}{l}\mathscr{O} \\
\dot{0}\end{array}$ & $\begin{array}{l}1 \\
0 \\
0\end{array}$ & $\stackrel{1}{\circ}$ & & $\begin{array}{l}0 \\
0 \\
0\end{array}$ & & $\stackrel{120}{\stackrel{10}{0}}$ & & $\begin{array}{l}1 \\
0 \\
0\end{array}$ & $\begin{array}{l}\dot{g} \\
\dot{0}\end{array}$ & $\begin{array}{l}\infty \\
0 \\
0\end{array}$ & $\begin{array}{l}\infty \\
0 \\
0\end{array}$ & \\
\hline$\approx$ & & & $\stackrel{10}{0}$ & 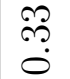 & $\begin{array}{l}\stackrel{20}{0} \\
\stackrel{0}{0}\end{array}$ & $\begin{array}{l}\infty \\
\stackrel{\sim}{0}\end{array}$ & $\stackrel{\mathrm{N}}{\stackrel{2}{0}}$ & & $\vec{F}$ & & $\begin{array}{l}\stackrel{\Im}{2} \\
\stackrel{0}{0}\end{array}$ & & $\stackrel{\sim}{\stackrel{2}{0}}$ & $\stackrel{\stackrel{H}{N}}{0}$ & 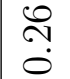 & 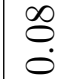 & \\
\hline S & & & 苞 & $\underset{\dot{0}}{\stackrel{F}{0}}$ & $\stackrel{\stackrel{\rho}{\rightleftarrows}}{\stackrel{0}{0}}$ & $\begin{array}{l}\vec{\jmath} \\
0\end{array}$ & $\stackrel{20}{\longrightarrow}$ & & $\begin{array}{l}\vec{\infty} \\
0 \\
0\end{array}$ & & $\overrightarrow{\tilde{o}}$ & & $\stackrel{\sim}{\circ}$ & $\begin{array}{l}\overparen{2} \\
ٌ \\
0\end{array}$ & $\vec{m}$ & $\begin{array}{l}\infty \\
\stackrel{1}{0} \\
0\end{array}$ & \\
\hline 5 & & & $\stackrel{\vartheta}{\hookrightarrow}$ & $\stackrel{0}{\stackrel{1}{0}}$ & $\stackrel{10}{\rightleftarrows}$ & $\begin{array}{l}\stackrel{0}{\prime} \\
0\end{array}$ & $\stackrel{1}{\circ}$ & & $\begin{array}{l}0 \\
0 \\
0\end{array}$ & & $\begin{array}{l}\infty \\
0 \\
0\end{array}$ & & $\begin{array}{l}1 \\
0 \\
0\end{array}$ & $\begin{array}{l}\dot{g} \\
\dot{0}\end{array}$ & $\stackrel{0}{1}$ & $\stackrel{m}{\longrightarrow}$ & \\
\hline $\overrightarrow{8}$ & & & i⿱乛龰 & $\overrightarrow{7}$ & $\vec{F}$ & $\stackrel{?}{\stackrel{0}{0}}$ & $\begin{array}{l}\hat{O} \\
0\end{array}$ & & ?ִ & & $\stackrel{\infty}{\infty}$ & & $\begin{array}{l}\stackrel{0}{:} \\
\stackrel{0}{0}\end{array}$ & $\begin{array}{l}\infty \\
0 \\
0\end{array}$ & $\stackrel{0}{1}$ & \begin{tabular}{l}
$\infty$ \\
\multirow{1}{*}{}
\end{tabular} & \\
\hline$\vec{\sigma}$ & & & - & $\neg$ & -1 & - & $\neg$ & & -1 & & $\neg$ & & - & $\neg$ & - & -1 & \\
\hline 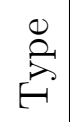 & $\stackrel{I}{\sim}$ & ㄱ. & $\stackrel{i}{\stackrel{2}{\sim}}$ & $\stackrel{\vec{q}}{\stackrel{P}{\sim}}$ & $\underset{+}{\stackrel{H}{H}}$ & $\underset{\sim}{\sim}$ & $\vec{I}$ & $\underset{F}{\vec{F}}$ & $\stackrel{i}{a}$ & 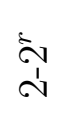 & 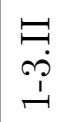 & 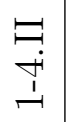 & $\underset{\sim}{\vec{d}}$ & تُ & $\stackrel{\sim}{\stackrel{\sim}{\infty}}$ & 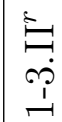 & $\stackrel{\xi}{+}$ \\
\hline
\end{tabular}




\begin{tabular}{|c|c|c|c|c|c|c|c|c|}
\hline$\frac{\widehat{g}}{\xi}$ & 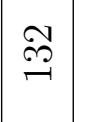 & $\overrightarrow{\mathscr{\vartheta}}$ & $\stackrel{m}{\Rightarrow}$ & $\overrightarrow{0}$ & 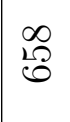 & 知 & $\underset{+}{\stackrel{\leftrightarrow}{9}}$ & I \\
\hline$\frac{\overparen{\pi}}{\frac{50}{\sigma 0}}$ & $\begin{array}{l}20 \\
i\end{array}$ & $\begin{array}{l}\infty \\
\stackrel{i}{i}\end{array}$ & $\stackrel{\leftrightarrow}{\mathrm{i}}$ & $\stackrel{\vec{i}}{\mathrm{i}}$ & $\begin{array}{l}\infty \\
i\end{array}$ & $\stackrel{L D}{\sim}$ & 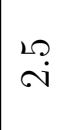 & $\stackrel{0}{\sim}$ \\
\hline $0^{20}$ & & & & & 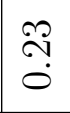 & & & $\stackrel{\not}{\sim}$ \\
\hline$\stackrel{\leftrightarrow}{\&}$ & & & & & 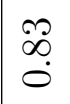 & & & $\begin{array}{l}\hat{N} \\
\stackrel{0}{0}\end{array}$ \\
\hline 80 & & & & & $\stackrel{0}{\stackrel{0}{0}}$ & & & $\stackrel{9}{\stackrel{0}{0}}$ \\
\hline$\vec{b}$ & & & & 吕 & 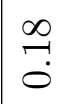 & & ָ̃ & $\stackrel{9}{\rightleftarrows}$ \\
\hline ஓ્ટ & & & & $\underset{\substack{N \\
0}}{0}$ & 茼 & & $\stackrel{\substack{0 \\
1 \\
0}}{0}$ & 苚 \\
\hline تే & & & & $\stackrel{2}{\stackrel{0}{0}}$ & $\stackrel{9}{\stackrel{0}{0}}$ & & $\stackrel{1}{\circ}$ & 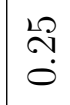 \\
\hline$b^{\infty}$ & & & ๑ొ & $\begin{array}{l}0 \\
-1 \\
0\end{array}$ & $\stackrel{\sim}{\stackrel{1}{0}}$ & $\begin{array}{l}\infty \\
\overrightarrow{0} \\
\overrightarrow{0}\end{array}$ & 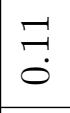 & $\begin{array}{l}\infty \\
0 \\
0\end{array}$ \\
\hline 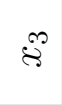 & & & $\stackrel{8}{\circ}$ & $\stackrel{0}{\mathscr{H}}$ & $\stackrel{\Lambda}{\tilde{\sigma}}$ & $\begin{array}{l}\stackrel{12}{2} \\
0 \\
0\end{array}$ & 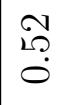 & $\stackrel{2}{2}$ \\
\hline 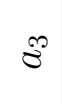 & & & $\stackrel{109}{\stackrel{0}{0}}$ & 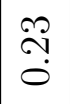 & 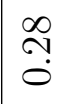 & $\vec{\jmath}$ & શָ & 号 \\
\hline$\tilde{6}$ & & 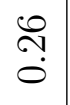 & $\stackrel{2}{?}$ & $\stackrel{8}{8}$ & $\stackrel{1}{0}$ & $\stackrel{20}{\longrightarrow}$ & $\stackrel{?}{\stackrel{1}{0}}$ & $\stackrel{?}{0}$ \\
\hline$\underset{\&}{*}$ & & $\begin{array}{l}R \\
0 \\
0\end{array}$ & $\underset{0}{F}$ & 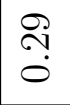 & 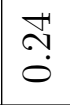 & $\stackrel{\leftrightarrow}{\stackrel{\bullet}{0}}$ & $\stackrel{8}{8}$ & $\stackrel{\infty}{\stackrel{0}{0}}$ \\
\hline $\mathbb{S}$ & & $\stackrel{?}{\stackrel{0}{0}}$ & స̆ & 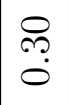 & ๙ֶ. & 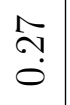 & $\stackrel{\stackrel{2}{0}}{\stackrel{N}{0}}$ & $\stackrel{\vec{N}}{\mathrm{~S}}$ \\
\hline 5 & $\begin{array}{c}0 \\
10 \\
0 \\
0\end{array}$ & 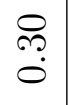 & $\stackrel{1}{\circ}$ & $\stackrel{3}{\stackrel{0}{0}}$ & $\vec{\exists}$ & $\stackrel{\Re}{\stackrel{\vartheta}{0}}$ & $\vec{J}$ & $\stackrel{\Im}{\dddot{3}}$ \\
\hline$\vec{\sigma}$ & $\stackrel{I}{\mathfrak{0}}$ & $\stackrel{\stackrel{\leftrightarrow}{~}}{\mathrm{o}}$ & $\stackrel{1}{0}$ & $\stackrel{?}{\mathfrak{0}}$ & $\exists$ & $\underset{+}{F}$ & $\stackrel{m}{\stackrel{m}{\circ}}$ & 号 \\
\hline$\xi$ & $\neg$ & $\neg$ & $\neg$ & $\dashv$ & $\dashv$ & - & $\dashv$ & - \\
\hline & & & $\neg$ & $\Xi$ & $\Xi$ & $\neg$ & $\Xi$ & $\Xi$ \\
\hline & 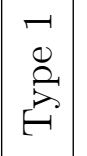 & 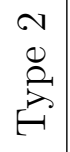 & \multicolumn{3}{|c|}{ 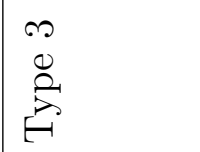 } & \multicolumn{3}{|l|}{ 峁 } \\
\hline
\end{tabular}




\begin{tabular}{|c|c|c|c|c|c|c|c|c|c|c|c|c|c|c|c|c|c|}
\hline$\underset{E}{\stackrel{0 \Omega}{\Xi}}$ & $\stackrel{\Omega}{\stackrel{B}{7}}$ & $\stackrel{8}{\varnothing}$ & & 今ి & 고 & $\ddot{8}$ & ๑̊ & & & & $\begin{array}{l}10 \\
\infty \\
10\end{array}$ & $\underset{0}{\infty}$ & ণิ & $\underset{0}{\stackrel{0}{0}}$ & $\underset{0}{0}$ & 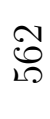 & $\begin{array}{l}\infty \\
\stackrel{1}{\rightarrow}\end{array}$ \\
\hline $\begin{array}{c}\frac{\pi}{\sigma 0} \\
\frac{50}{\sigma 0}\end{array}$ & $\stackrel{10}{\sim}$ & $\stackrel{\not t}{\sim}$ & & $\stackrel{10}{\mathrm{~N}}$ & $\begin{array}{l}0 \\
ن \\
ن\end{array}$ & $\begin{array}{l}\infty \\
i\end{array}$ & مُ & & & & $\underset{\infty}{0}$ & $\stackrel{\sim}{\sim}$ & $\stackrel{H}{\sim}$ & $\begin{array}{l}0 \\
\sim\end{array}$ & $\stackrel{10}{i}$ & $\stackrel{+}{\sim}$ & $\stackrel{\not t}{\sim}$ \\
\hline$b^{10}$ & & & & & & & & & & & $\stackrel{\mathscr{m}}{\stackrel{0}{0}}$ & \begin{tabular}{l}
$\infty$ \\
$\stackrel{\infty}{0}$ \\
\hdashline
\end{tabular} & 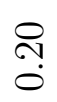 & $\stackrel{\infty}{\stackrel{2}{0}}$ & $\stackrel{\infty}{\stackrel{\infty}{0}}$ & $\stackrel{\rho}{\stackrel{0}{0}}$ & $\underset{\Xi}{\rightleftarrows}$ \\
\hline$\overbrace{0}^{10}$ & & & & & & & & & & & $\begin{array}{l}1 \\
\infty \\
0\end{array}$ & 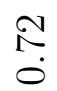 & $\begin{array}{l}\text { N } \\
\stackrel{\infty}{0}\end{array}$ & $\begin{array}{l}0 \\
\infty \\
0\end{array}$ & $\begin{array}{l}0 \\
\infty \\
0\end{array}$ & $\stackrel{R}{R}$ & $\begin{array}{l}\infty \\
\infty \\
0\end{array}$ \\
\hline 8 & & & & & & & & & & & Oo & 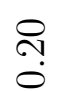 & $\underset{b}{\stackrel{H}{0}}$ & $\stackrel{\vec{H}}{\overrightarrow{0}}$ & 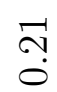 & $\stackrel{\vec{H}}{\stackrel{H}{0}}$ & $\stackrel{0}{\rightleftharpoons}$ \\
\hline$b^{+}$ & & & & $\ddot{0}$ & $\stackrel{N}{\stackrel{N}{0}}$ & 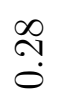 & $\stackrel{m}{\stackrel{m}{0}}$ & & & & $\stackrel{+}{\vec{H}}$ & $\stackrel{\infty}{\rightleftarrows}$ & $\stackrel{10}{\rightleftarrows}$ & $\begin{array}{l}0 \\
? \\
0\end{array}$ & $\begin{array}{l}0 \\
\stackrel{0}{0}\end{array}$ & $\vec{F}$ & $\stackrel{\leftrightarrow}{\stackrel{0}{0}}$ \\
\hline तु & & & & $\stackrel{?}{\stackrel{0}{0}}$ & $\stackrel{R}{0}$ & $\stackrel{i}{i}$ & $\begin{array}{l}\vec{\infty} \\
0\end{array}$ & & & & $\vec{\Gamma}$ & $\begin{array}{l}+1 \\
0 \\
0\end{array}$ & $\begin{array}{l}0 \\
0 \\
0\end{array}$ & $\stackrel{R}{R}$ & $\stackrel{?}{i}$ & بْ & $\stackrel{0}{0}$ \\
\hline है & & & & $\stackrel{10}{2}$ & $\stackrel{10}{\stackrel{20}{0}}$ & 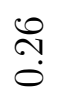 & ڤె. & & & & $\stackrel{\vec{N}}{0}$ & $\begin{array}{l}\infty \\
? \\
0\end{array}$ & $\stackrel{\leftrightarrow}{\leftrightarrow}$ & ?ִ & $\ddot{0}$ & 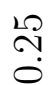 & $\stackrel{+}{0}$ \\
\hline$b^{m}$ & ? & $\begin{array}{l}\stackrel{?}{0} \\
\stackrel{0}{0}\end{array}$ & & $\begin{array}{l}\stackrel{0}{t} \\
\ddot{0}\end{array}$ & $\stackrel{\infty}{\stackrel{m}{0}}$ & $\stackrel{\stackrel{\sim}{Z}}{\stackrel{0}{0}}$ & 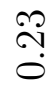 & & & & $\stackrel{\infty}{0}$ & $\stackrel{1}{0}$ & $\begin{array}{l}8 \\
\dot{0}\end{array}$ & $\stackrel{1}{\stackrel{1}{0}}$ & $\underset{0}{\vec{J}}$ & $\stackrel{2}{0}$ & $\stackrel{8}{8}$ \\
\hline జु & $\begin{array}{l}1 \\
0 \\
0\end{array}$ & $\begin{array}{l}0 \\
\ddot{0} \\
\dot{0}\end{array}$ & & $\begin{array}{l}0 \\
\stackrel{1}{0} \\
0\end{array}$ & $\begin{array}{l}0 \\
\stackrel{1}{0} \\
0\end{array}$ & $\begin{array}{l}\text { ?2 } \\
\text { ?2 }\end{array}$ & $\stackrel{10}{+}$ & & & & $\begin{array}{l}0 \\
1+ \\
0\end{array}$ & $\stackrel{10}{\stackrel{10}{0}}$ & $\begin{array}{l}0 \\
\stackrel{1}{0} \\
0\end{array}$ & $\begin{array}{l}10 \\
10 \\
0\end{array}$ & $\stackrel{0}{\stackrel{0}{0}}$ & $\begin{array}{l}0 \\
0 \\
0 \\
0\end{array}$ & m. \\
\hline ஓొ & $\stackrel{N}{\sim}$ & $\stackrel{\stackrel{f}{+}}{\circ}$ & & $\stackrel{\sim}{\stackrel{\sim}{\circ}}$ & $\stackrel{\sim}{\stackrel{N}{0}}$ & ?ִ & $\stackrel{\infty}{0}$ & & & & $\stackrel{N}{0}$ & 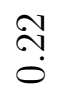 & ֶి & 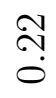 & $\stackrel{\infty}{\rightarrow}$ & ?ִ & $\stackrel{\stackrel{f}{f}}{\circ}$ \\
\hline$\sigma^{N}$ & $\stackrel{10}{\longrightarrow}$ & $\stackrel{0}{\stackrel{1}{0}}$ & & 궁 & $\underset{0}{Z}$ & $\stackrel{\infty}{0}$ & $\stackrel{\infty}{0}$ & & & & $\stackrel{\vec{t}}{\overrightarrow{0}}$ & 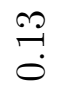 & $\begin{array}{l}1 \\
0 \\
0\end{array}$ & $\stackrel{\infty}{0}$ & $\stackrel{\infty}{0}$ & $\begin{array}{l}0 \\
\stackrel{0}{0}\end{array}$ & $\begin{array}{l}0 \\
\stackrel{0}{0}\end{array}$ \\
\hline से & $\stackrel{\vec{H}}{\stackrel{H}{0}}$ & $\ddot{n}$ & & ֶి & חి & $\stackrel{N}{\text { กิ }}$ & 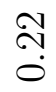 & & & & $\stackrel{\circ}{\stackrel{H}{0}}$ & $\stackrel{\text { me }}{0}$ & 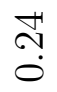 & 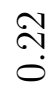 & $\begin{array}{l}\text { ஸे } \\
\text { o }\end{array}$ & $\stackrel{g}{0}$ & $\begin{array}{l}0 \\
\stackrel{0}{0}\end{array}$ \\
\hline$\tilde{\delta}$ & $\begin{array}{l}0 \\
0 ? \\
0\end{array}$ & $\stackrel{10}{\stackrel{10}{0}}$ & & 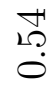 & $\stackrel{i}{0}$ & 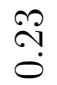 & ণै & & & & $\begin{array}{l}\infty \\
1 ? \\
0 \\
0\end{array}$ & 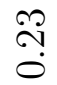 & 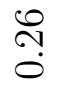 & 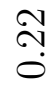 & $\stackrel{N}{o}$ & $\begin{array}{l}\infty \\
0 \\
0 \\
0\end{array}$ & $\stackrel{\sigma}{0}$ \\
\hline 5 & $\stackrel{m}{\ddot{0}}$ & $\stackrel{\vec{t}}{\ddot{0}}$ & & $\stackrel{F}{\circ}$ & $\stackrel{\mathscr{Y}}{\rightleftarrows}$ & $\ddot{Z}$ & $\stackrel{8}{0}$ & & & & $\ddot{f}$ & $\stackrel{m}{\stackrel{m}{0}}$ & $\stackrel{\circ}{\stackrel{0}{0}}$ & $\begin{array}{l}0 \\
\stackrel{0}{0} \\
0\end{array}$ & $\ddot{\sigma}$ & $\ddot{Z}$ & 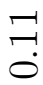 \\
\hline$\vec{\psi}$ & $\stackrel{\stackrel{N}{f}}{\stackrel{0}{0}}$ & $\stackrel{m}{\stackrel{m}{0}}$ & & $\stackrel{F}{\vec{\sigma}}$ & $\stackrel{\rightleftarrows}{\rightleftarrows}$ & $\ddot{\rightleftarrows}$ & $\begin{array}{l}\mathscr{0} \\
\dot{0}\end{array}$ & & & & $\begin{array}{l}\stackrel{0}{1} \\
\dot{0}\end{array}$ & $\stackrel{ت}{\rightleftarrows}$ & $\begin{array}{l}\mathscr{O} \\
\stackrel{0}{0}\end{array}$ & $\stackrel{\stackrel{N}{Z}}{\stackrel{0}{0}}$ & $\begin{array}{l}\stackrel{0}{1} \\
\dot{0}\end{array}$ & ?ִ & $\begin{array}{l}\mathscr{\bullet} \\
\stackrel{0}{0}\end{array}$ \\
\hline$\vec{\sigma}$ & - & $\neg$ & & $\neg$ & 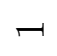 & $\neg$ & $\neg$ & & & & - & $\neg$ & $\neg$ & $\neg$ & - & $\neg$ & - \\
\hline$\stackrel{0}{\stackrel{2}{2}}$ & $\stackrel{N}{\perp}$ & ' & $\stackrel{i}{1}$ & $\vec{P}$ & 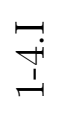 & $\begin{array}{l}\text { N } \\
\text { N่ }\end{array}$ & $\frac{7}{\stackrel{1}{c}}$ & 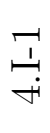 & P. & $\begin{array}{l}\stackrel{\sim}{a} \\
\stackrel{1}{N}\end{array}$ & $\underset{\dot{P}}{ت}$ & $\underset{+}{\stackrel{\longrightarrow}{+}}$ & $\begin{array}{l}\vec{\leftrightarrow} \\
\vec{d} \\
\vec{N}\end{array}$ & تُ & $\frac{\stackrel{T}{\perp}}{\stackrel{0}{\infty}}$ & 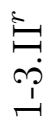 & $\stackrel{\nu}{\exists}$ \\
\hline
\end{tabular}




\begin{tabular}{|c|c|c|c|c|c|c|c|c|}
\hline$\frac{\widehat{g}}{\xi}$ & $\stackrel{\stackrel{N}{\mathscr{D}}}{\sim}$ & 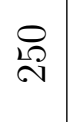 & 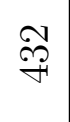 & $\begin{array}{l}20 \\
8 \\
10\end{array}$ & $\frac{2.2}{12}$ & $\begin{array}{l}\ddot{8} \\
\because\end{array}$ & 荅 & 8 \\
\hline$\frac{\overparen{\pi}}{\frac{50}{\sigma 0}}$ & $\stackrel{\vec{T}}{\mathrm{r}}$ & $\begin{array}{l}\dot{\sim} \\
\stackrel{N}{*}\end{array}$ & $\hat{\imath}$ & $\stackrel{\sim}{i}$ & $\begin{array}{l}0 \\
i\end{array}$ & $\stackrel{\leftrightarrow}{i}$ & $\stackrel{\vec{i}}{\stackrel{i}{i}}$ & $\stackrel{\leftrightarrow}{\sim}$ \\
\hline$b^{20}$ & & & & & શiv & & & $\stackrel{\stackrel{\sim}{N}}{0}$ \\
\hline$\stackrel{\leftrightarrow}{\&}$ & & & & & $\begin{array}{l}\infty \\
1 \\
0 \\
0\end{array}$ & & & $\begin{array}{l}\hat{N} \\
\stackrel{0}{0}\end{array}$ \\
\hline 80 & & & & & $\stackrel{\sim}{\stackrel{2}{0}}$ & & & $\stackrel{20}{\stackrel{0}{0}}$ \\
\hline$\vec{b}$ & & & & 尺ै & $\begin{array}{l}0 \\
0 \\
0\end{array}$ & & Ñ & $\stackrel{m}{\longrightarrow}$ \\
\hline ஓ્ટ & & & & $\stackrel{8}{8}$ & 茼 & & 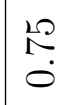 & 苚 \\
\hline تే & & & & 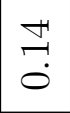 & $\begin{array}{l}\infty \\
\stackrel{0}{0}\end{array}$ & & $\stackrel{10}{-1}$ & 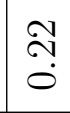 \\
\hline$b^{\infty}$ & & & $\begin{array}{l}\stackrel{\curvearrowright}{~} \\
\stackrel{0}{~}\end{array}$ & $\begin{array}{l}0 \\
-1 \\
0\end{array}$ & $\stackrel{\sim}{\stackrel{1}{0}}$ & $\begin{array}{l}\infty \\
\overrightarrow{0} \\
\overrightarrow{0}\end{array}$ & 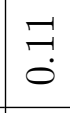 & $\begin{array}{l}\infty \\
0 \\
0\end{array}$ \\
\hline 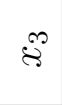 & & & $\stackrel{\bullet}{\bullet}$ & $\stackrel{f}{\sharp}$ & 紫 & $\begin{array}{l}\stackrel{R}{2} \\
0 \\
0\end{array}$ & $\begin{array}{l}0 \\
\stackrel{1}{0} \\
0\end{array}$ & $\stackrel{2}{2}$ \\
\hline 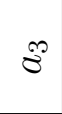 & & & $\begin{array}{l}0 \\
\stackrel{0}{0}\end{array}$ & ָ̃ & Lొ & \begin{tabular}{l}
$\stackrel{9}{2}$ \\
\hdashline
\end{tabular} & $\stackrel{H}{N}$ & $\stackrel{\stackrel{2}{~}}{\stackrel{0}{0}}$ \\
\hline$\tilde{6}$ & & 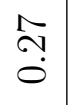 & $\stackrel{?}{\stackrel{0}{0}}$ & $\stackrel{8}{8}$ & $\stackrel{1}{0}$ & $\stackrel{\vec{H}}{-}$ & $\stackrel{0}{0}$ & $\stackrel{\infty}{0}$ \\
\hline स्षे & & $\stackrel{\mathscr{O}}{0}$ & $\underset{0}{ت}$ & 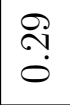 & $\stackrel{\Re}{\stackrel{⿰}{0}}$ & $\stackrel{1}{\breve{0}}$ & $\stackrel{g}{0}$ & 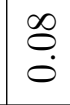 \\
\hline $\mathbb{S}$ & & $\stackrel{?}{\stackrel{0}{0}}$ & $\begin{array}{l}\stackrel{0}{~} \\
\stackrel{0}{0}\end{array}$ & શָ & શָ & $\underset{0}{\stackrel{4}{0}}$ & $\stackrel{\stackrel{2}{2}}{\stackrel{2}{o}}$ & $\stackrel{\vec{N}}{\mathrm{~S}}$ \\
\hline 5 & 年 & $\stackrel{\substack{0 \\
\hdashline}}{0}$ & $\stackrel{1}{\breve{0}}$ & 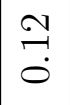 & $\stackrel{0}{\stackrel{1}{0}}$ & $\stackrel{\Re}{\stackrel{\vartheta}{0}}$ & $\stackrel{20}{\longrightarrow}$ & $\stackrel{9}{\stackrel{0}{0}}$ \\
\hline$\vec{\sigma}$ & $\begin{array}{l}F \\
\dot{0}\end{array}$ & 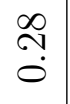 & $\stackrel{\infty}{\stackrel{0}{0}}$ & $\stackrel{m}{\stackrel{9}{0}}$ & $\stackrel{0}{\stackrel{0}{0}}$ & $\stackrel{?}{\stackrel{0}{0}}$ & $\overrightarrow{\ddot{\theta}}$ & 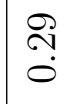 \\
\hline$\vec{\sigma}$ & - & - & $\rightarrow$ & $\neg$ & $\neg$ & - & - & - \\
\hline & & & $\neg$ & 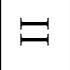 & $\Xi$ & $\neg$ & $\Xi$ & $\Xi$ \\
\hline & 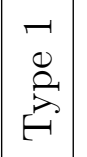 & 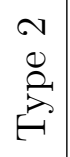 & \multicolumn{3}{|l|}{ ڤ્ } & \multicolumn{3}{|l|}{ 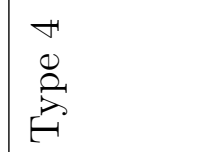 } \\
\hline
\end{tabular}




\begin{tabular}{|c|c|c|c|c|c|c|c|c|c|c|c|c|c|c|c|c|c|}
\hline$\frac{\tilde{g}}{\tilde{G}}$ & $\begin{array}{l}\infty \\
\stackrel{\infty}{\rightarrow}\end{array}$ & $\infty$ & $\begin{array}{l}\text { ND } \\
\text { 敌 }\end{array}$ & 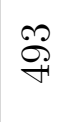 & 总 & $\begin{array}{l}\not{D} \\
\stackrel{D}{\infty}\end{array}$ & & $\stackrel{0}{\not{f}}$ & $\stackrel{0}{10}$ & $\underset{\Delta B}{\vec{H}}$ & \begin{tabular}{l}
$\infty$ \\
\hdashline \\
\hdashline
\end{tabular} & 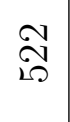 & $\begin{array}{l}20 \\
80 \\
10\end{array}$ & $\stackrel{\substack{\infty \\
\infty}}{\infty}$ & 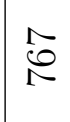 & $\overrightarrow{D H}$ & \\
\hline $\begin{array}{l}\frac{\pi}{30} \\
\frac{30}{\sigma 0}\end{array}$ & $\stackrel{\vec{i}}{\mathrm{i}}$ & $\stackrel{\infty}{\underset{i}{i}}$ & $\stackrel{\mathcal{N}}{\mathrm{N}}$ & $\stackrel{H}{i}$ & $\stackrel{\vec{i}}{i}$ & $\begin{array}{l}\infty \\
i\end{array}$ & & $\vec{a}$ & $\stackrel{H}{i}$ & $\stackrel{\vec{N}}{\mathrm{~N}}$ & $\stackrel{m}{i}$ & $\overrightarrow{\mathrm{N}}$ & $\stackrel{10}{i}$ & in & $\hat{a}$ & $\stackrel{\mathcal{N}}{\mathrm{N}}$ & \\
\hline 6 & & & & & & & & & & & $\begin{array}{l}8 \\
0 \\
0\end{array}$ & $\underset{0}{\sharp}$ & 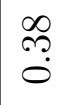 & $\vec{~}$ & $\stackrel{ }{0}$ & $\stackrel{0}{\stackrel{0}{0}}$ & \\
\hline$\stackrel{20}{8}$ & & & & & & & & & & & $\begin{array}{l}R \\
T \\
0\end{array}$ & $\begin{array}{l}10 \\
\infty \\
0 \\
0\end{array}$ & $\begin{array}{l}0 \\
\infty \\
0 \\
0\end{array}$ & $\begin{array}{l}0 \\
\stackrel{0}{1} \\
0\end{array}$ & $\begin{array}{l}\infty \\
\infty \\
\infty\end{array}$ & $\begin{array}{l}1 \\
\infty \\
0 \\
0\end{array}$ & \\
\hline 8 & & & & & & & & & & & 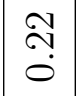 & $\stackrel{?}{\rightleftarrows}$ & $\stackrel{H}{\rightleftarrows}$ & $\stackrel{+}{\stackrel{1}{0}}$ & $\stackrel{12}{-10}$ & ○. & \\
\hline $\overrightarrow{0}^{+}$ & & & & $\begin{array}{l}0 \\
\stackrel{0}{0} \\
\stackrel{0}{0}\end{array}$ & 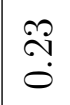 & 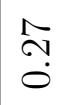 & & \begin{tabular}{|l}
$\infty$ \\
$\stackrel{\infty}{0}$ \\
$\stackrel{0}{0}$
\end{tabular} & Nָ & $\begin{array}{l}0 \\
\stackrel{1}{0}\end{array}$ & $\begin{array}{l}\hat{0} \\
\stackrel{0}{0}\end{array}$ & $\begin{array}{l}\infty \\
0 \\
0\end{array}$ & $\stackrel{\sim}{\stackrel{0}{0}}$ & $\begin{array}{l}1 \\
0 \\
0\end{array}$ & 号 & $\stackrel{\mathcal{F}}{3}$ & \\
\hline ¿ & & & & $\begin{array}{l}\mathscr{\infty} \\
\infty \\
0 \\
0\end{array}$ & 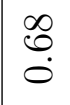 & $\begin{array}{l}R \\
0 \\
0\end{array}$ & & : & $\begin{array}{l}\text { 송 } \\
0\end{array}$ & $\begin{array}{l}\infty \\
\stackrel{1}{0} \\
0\end{array}$ & $\begin{array}{l}\mathfrak{N} \\
\mathfrak{0}\end{array}$ & $\stackrel{8}{0}$ & $\stackrel{?}{\stackrel{P}{2}}$ & O̊. & $\begin{array}{l}R \\
0 \\
0\end{array}$ & $\stackrel{R}{0}$ & \\
\hline కt & & & & $\stackrel{8}{8}$ & ๙ึ. & $\stackrel{1}{\breve{0}}$ & & Ö. & $\stackrel{\nexists}{*}$ & $\stackrel{20}{\overparen{0}}$ & $\begin{array}{l}8 \\
0 \\
0\end{array}$ & $\stackrel{+}{\stackrel{\leftrightarrow}{0}}$ & 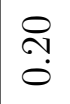 & స̃. & $\begin{array}{l}\stackrel{2}{2} \\
\stackrel{0}{0}\end{array}$ & $\stackrel{9}{\longrightarrow}$ & \\
\hline$\dot{b}^{\infty}$ & $\begin{array}{l}\infty \\
\stackrel{\infty}{0} \\
\stackrel{0}{0}\end{array}$ & $\stackrel{\vec{N}}{\stackrel{0}{0}}$ & 尺̃ & $\stackrel{\oplus}{\stackrel{9}{0}}$ & 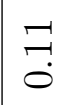 & $\stackrel{10}{\stackrel{10}{0}}$ & & $\stackrel{8}{0}$ & $\vec{F}$ & $\begin{array}{c}\stackrel{0}{1} \\
0 \\
0\end{array}$ & 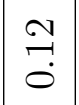 & $\stackrel{0}{\stackrel{1}{0}}$ & $\stackrel{\oplus}{\stackrel{0}{0}}$ & 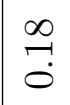 & $=$ & $\stackrel{8}{0}$ & \\
\hline$\stackrel{\leftrightarrow}{\oplus}$ & $\stackrel{\vec{L}}{\stackrel{2}{0}}$ & $\stackrel{12}{10}$ & $\begin{array}{l}10 \\
\infty \\
0 \\
0\end{array}$ & $\begin{array}{l}0 \\
L \\
0 \\
0\end{array}$ & 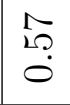 & 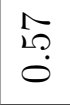 & & $\begin{array}{l}0 \\
\stackrel{2}{0} \\
0\end{array}$ & $\stackrel{8}{0}$ & 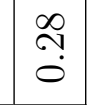 & $\begin{array}{l}F \\
\dot{0}\end{array}$ & $\stackrel{\vec{D}}{\Delta}$ & 落 & $\stackrel{2}{\stackrel{2}{0}}$ & 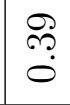 & $\stackrel{0}{0}$ & \\
\hline $\mathbb{8}$ & 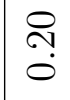 & $\widetilde{\widetilde{o}}$ & $\stackrel{m}{\breve{0}}$ & 艿 & 望 & $\underset{\dot{0}}{\ddot{H}}$ & & $\stackrel{0}{\stackrel{0}{0}}$ & 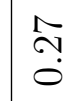 & 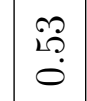 & $\vec{s}$ & $\begin{array}{l}\infty \\
\infty \\
0\end{array}$ & 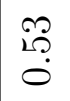 & $\begin{array}{l}0 \\
-1 \\
0\end{array}$ & $\stackrel{m}{\stackrel{2}{0}}$ & $\stackrel{\sim}{N}$ & \\
\hline$\sigma^{N}$ & $\stackrel{10}{-10}$ & $\stackrel{m}{\overrightarrow{0}}$ & $\mid \begin{array}{l}\infty \\
\stackrel{0}{0} \\
0\end{array}$ & $\overrightarrow{7}$ & $\stackrel{8}{\circ}$ & $\stackrel{\infty}{\stackrel{0}{0}}$ & & مُ & ?. & 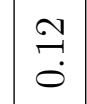 & $\begin{array}{l}\infty \\
0 \\
0 \\
0\end{array}$ & $\stackrel{m}{\stackrel{0}{0}}$ & $\stackrel{8}{\circ}$ & م. & مُ & $\stackrel{\vec{H}}{-}$ & \\
\hline జ્త & $\stackrel{\Re}{\stackrel{P}{0}}$ & ֶֻ? & $\mid$\begin{tabular}{ll}
20 \\
\hdashline
\end{tabular} & 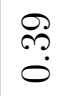 & 号 & 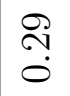 & & 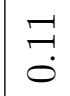 & $\stackrel{8}{0}$ & $\vec{F}$ & $\mid \begin{array}{c}0 \\
\tilde{o} \\
\stackrel{0}{0}\end{array}$ & 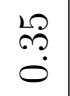 & $\stackrel{H}{\stackrel{H}{0}}$ & શิ & $\stackrel{\vec{N}}{\stackrel{0}{0}}$ & $\stackrel{\mathcal{I}}{?}$ & \\
\hline $\mathfrak{S}$ & $\stackrel{\substack{0 \\
0}}{0}$ & $\stackrel{1}{0}$ & 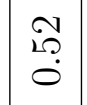 & i⿱乛龰 & $\stackrel{9}{\stackrel{0}{0}}$ & 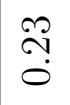 & & 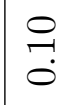 & $\stackrel{?}{?}$ & 思 & $\begin{array}{l}0 \\
10 \\
0 \\
0\end{array}$ & $\stackrel{\infty}{\stackrel{\infty}{0}}$ & $\vec{\jmath}$ & 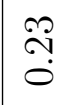 & 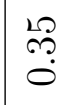 & $\begin{array}{l}\infty \\
\infty \\
0\end{array}$ & \\
\hline$\vec{b}$ & $\stackrel{\vec{H}}{\stackrel{4}{0}}$ & ? & 궁 & $\underset{⿱}{+}$ & $\stackrel{7}{\stackrel{7}{0}}$ & $\stackrel{\sim}{\stackrel{2}{0}}$ & & $\begin{array}{l}0 \\
0 \\
0\end{array}$ & $\stackrel{20}{9}$ & $\stackrel{m}{\stackrel{0}{0}}$ & $\begin{array}{l}\stackrel{0}{1} \\
0 \\
0\end{array}$ & $\stackrel{m}{\stackrel{0}{0}}$ & $\stackrel{\sim}{\stackrel{7}{0}}$ & $\stackrel{\infty}{\infty}$ & 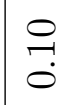 & $\stackrel{\vec{H}}{-}$ & \\
\hline $\overrightarrow{\&}$ & $\stackrel{\Im}{\stackrel{3}{0}}$ & Эั) & $\begin{array}{c}\overrightarrow{2} \\
20 \\
0 \\
0\end{array}$ & $\vec{J}$ & $\stackrel{m}{\stackrel{0}{0}}$ & $\vec{l}=\overrightarrow{1}$ & & $\overrightarrow{\tilde{S}}$ & \begin{tabular}{c}
$\infty$ \\
$\dddot{0}$ \\
\hdashline \\
0
\end{tabular} & $\mid \begin{array}{c}0 \\
\stackrel{3}{a} \\
0\end{array}$ & $\stackrel{\mathfrak{7}}{\stackrel{0}{0}}$ & $\overrightarrow{7}$ & $\stackrel{8}{8}$ & $\begin{array}{l}\infty \\
0 \\
0\end{array}$ & 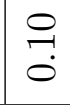 & J্ & \\
\hline$\vec{\sigma}$ & - & - & -1 & $\neg$ & $\neg$ & $\neg$ & & - & 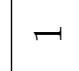 & $\rightarrow$ & $\neg$ & -1 & $\neg$ & - & - & - & \\
\hline 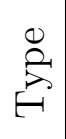 & $\stackrel{\sim}{\sim}$ & $\overrightarrow{\lrcorner}$ & ì & $\underset{\stackrel{P}{\longrightarrow}}{\stackrel{\longrightarrow}{\longrightarrow}}$ & $\stackrel{\overrightarrow{+}}{\stackrel{+}{+}}$ & $\stackrel{\sim}{\sim}$ & $\underset{\infty}{\stackrel{a}{a}}$ & $\stackrel{\rightleftarrows}{\stackrel{\perp}{+}}$ & $\stackrel{\vec{H}}{\stackrel{P}{P}}$ & خ̀ & 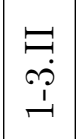 & $\underset{\stackrel{+}{\rightleftarrows}}{\stackrel{F}{+}}$ & $\underset{\sim}{\stackrel{p}{\sim}}$ & $\underset{+}{\stackrel{+}{\sim}}$ & $\stackrel{\sim}{\stackrel{\sim}{\perp}}$ & $\begin{array}{l}\tilde{H} \\
\stackrel{P}{P} \\
\dot{P}\end{array}$ & 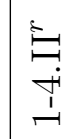 \\
\hline
\end{tabular}




\begin{tabular}{|c|c|c|c|c|c|c|c|c|}
\hline$\frac{\widehat{g}}{\xi}$ & $\stackrel{\stackrel{\mathscr{D}}{\Im}}{\stackrel{9}{9}}$ & $\begin{array}{l}20 \\
\stackrel{2}{\sim} \\
\stackrel{2}{*}\end{array}$ & $\stackrel{ }{\exists}$ & 窝 & 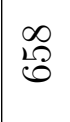 & 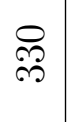 & 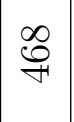 & $\begin{array}{l}\infty \\
\stackrel{0}{\circ}\end{array}$ \\
\hline$\frac{\overparen{\pi}}{\frac{50}{\sigma 0}}$ & $\vec{i}$ & $\hat{\sim}$ & $\begin{array}{l}\infty \\
i \\
i\end{array}$ & $\begin{array}{l}\infty \\
i \\
i\end{array}$ & $\grave{i}$ & $\stackrel{\leftrightarrow}{\mathrm{i}}$ & $\stackrel{\vec{i}}{\mathrm{i}}$ & 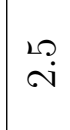 \\
\hline $0^{20}$ & & & & & 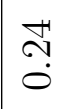 & & & $\stackrel{\sim}{\tilde{\sigma}}$ \\
\hline$\stackrel{\leftrightarrow}{\&}$ & & & & & 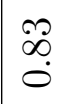 & & & $\begin{array}{l}\stackrel{\infty}{\infty} \\
\stackrel{0}{0}\end{array}$ \\
\hline 10 & & & & & $\stackrel{0}{\circ}$ & & & $\stackrel{\sim}{\stackrel{0}{0}}$ \\
\hline$\vec{b}$ & & & & $\vec{\sigma}$ & $\stackrel{9}{\leftrightarrows}$ & & 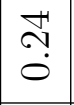 & $\stackrel{\vec{H}}{0}$ \\
\hline ङ & & & & 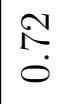 & 总 & & $\begin{array}{l}\overrightarrow{1} \\
\vdots \\
0\end{array}$ & $\stackrel{8}{0}$ \\
\hline تే & & & & 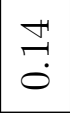 & ĩ & & $\stackrel{ }{\stackrel{1}{0}}$ & $\stackrel{\stackrel{2}{2}}{\stackrel{0}{0}}$ \\
\hline$b^{\infty}$ & & & $\vec{m}$ & 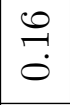 & $\stackrel{1}{3}$ & $\stackrel{9}{\leftrightarrows}$ & $\overrightarrow{7}$ & $\stackrel{8}{0}$ \\
\hline$\approx$ & & & $\begin{array}{l}n \\
0 \\
0\end{array}$ & $\stackrel{0}{\mathscr{H}}$ & $\stackrel{\Lambda}{\tilde{\sigma}}$ & $\begin{array}{l}\stackrel{R}{2} \\
0 \\
0\end{array}$ & 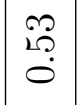 & 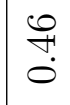 \\
\hline 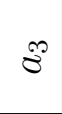 & & & $\begin{array}{l}0 \\
\stackrel{0}{0}\end{array}$ & 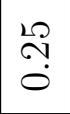 & $\begin{array}{l}\infty \\
\stackrel{N}{0} \\
0\end{array}$ & $\begin{array}{l}\infty \\
\stackrel{\infty}{*}\end{array}$ & 尺े & 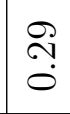 \\
\hline$\tilde{6}$ & & $\stackrel{2}{\stackrel{2}{0}}$ & $\stackrel{m}{\stackrel{0}{0}}$ & $\stackrel{8}{8}$ & $\stackrel{5}{0}$ & $\stackrel{\infty}{\stackrel{0}{0}}$ & $\stackrel{0}{\stackrel{0}{0}}$ & $\stackrel{8}{0}$ \\
\hline स्षे & & $\begin{array}{l}R \\
0 \\
0\end{array}$ & $\underset{0}{\stackrel{F}{0}}$ & 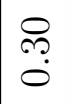 & 总 & $\stackrel{m}{\stackrel{0}{0}}$ & $\stackrel{0}{1}$ & $\stackrel{\infty}{\stackrel{0}{0}}$ \\
\hline $\mathbb{S}$ & & 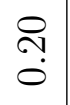 & 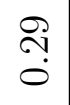 & 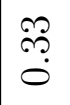 & ֶֶ. & $\stackrel{\sim}{\circ}$ & 今ึ & 号 \\
\hline 5 & 总 & 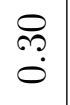 & $\stackrel{1}{\breve{0}}$ & $\stackrel{3}{\stackrel{0}{0}}$ & $\vec{\exists}$ & $\stackrel{\vec{H}}{\mathrm{O}}$ & $\stackrel{1}{\stackrel{1}{0}}$ & $\stackrel{9}{\stackrel{0}{0}}$ \\
\hline$\vec{\sigma}$ & $\begin{array}{l}P \\
0 \\
0\end{array}$ & $\stackrel{\stackrel{\leftrightarrow}{~}}{\mathrm{o}}$ & $\stackrel{1}{\breve{0}}$ & $\stackrel{m}{\stackrel{9}{0}}$ & $\exists$ & $\stackrel{?}{\stackrel{0}{0}}$ & $\vec{s}$ & 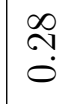 \\
\hline$\vec{\sigma}$ & - & - & $\rightarrow$ & $\neg$ & $\neg$ & - & $\neg$ & - \\
\hline & & & $\neg$ & $\Xi$ & $\Xi$ & $\neg$ & $\Xi$ & $\Xi$ \\
\hline & 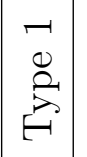 & 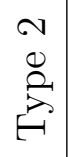 & \multicolumn{3}{|l|}{ 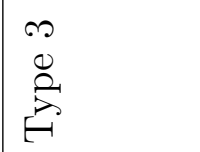 } & \multicolumn{3}{|l|}{ 峁 } \\
\hline
\end{tabular}




\begin{tabular}{|c|c|c|c|c|c|c|c|c|c|c|c|c|c|c|c|c|c|}
\hline$\underset{E}{\stackrel{\pi n}{\Xi}}$ & ळे & $\begin{array}{l}0 \\
\frac{1}{20}\end{array}$ & $\begin{array}{l}\infty \\
\text { న } \\
\text { న }\end{array}$ & ¿্ర & $\stackrel{\infty}{\stackrel{\infty}{1}}$ & 志 & $\mathscr{8}$ & & $\begin{array}{l}1 \\
\infty \\
10\end{array}$ & & ภู & $\begin{array}{l}10 \\
0 \\
0\end{array}$ & 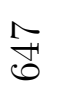 & $\stackrel{m}{\Gamma}$ & $\begin{array}{l}10 \\
12 \\
12\end{array}$ & $\stackrel{+}{8}$ & $\stackrel{\overbrace{}}{\stackrel{ి}{N}}$ \\
\hline $\begin{array}{l}\frac{\sqrt{\pi}}{\sqrt[6]{0}} \\
\frac{\sigma}{\sigma}\end{array}$ & $\stackrel{\vec{i}}{i}$ & $\stackrel{0}{0}$ & $\begin{array}{l}\infty \\
\text { o }\end{array}$ & $\stackrel{\sim}{\sim}$ & $\stackrel{\leftrightarrow}{\sim}$ & $\stackrel{\sim}{\sim}$ & $\stackrel{\sim}{\sim}$ & & $\tilde{\sim}$ & & 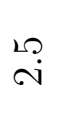 & $\ddot{\sim}$ & 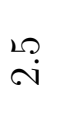 & $\stackrel{\leftrightarrow}{\sim}$ & $\stackrel{+}{\Delta}$ & $\begin{array}{l}L \mathcal{L} \\
\mathrm{N}\end{array}$ & $\stackrel{\circ}{\circ}$ \\
\hline$b^{20}$ & & & & & & & & & & & $\stackrel{\stackrel{L}{~}}{0}$ & ڤै & $\ddot{\infty}$ & $\stackrel{H}{\stackrel{N}{0}}$ & 吕 & $\stackrel{N}{N}$ & $\stackrel{\circ}{\stackrel{1}{0}}$ \\
\hline$\stackrel{20}{\&}$ & & & & & & & & & & & $\begin{array}{l}\infty \\
\infty \\
0\end{array}$ & $\begin{array}{l}\stackrel{0}{\infty} \\
0 \\
0\end{array}$ & 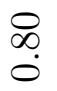 & $\begin{array}{l}\infty \\
\infty \\
0\end{array}$ & $\begin{array}{l}10 \\
\infty \\
0 \\
0\end{array}$ & $\stackrel{R}{R}$ & $\begin{array}{l}10 \\
\infty \\
0 \\
0\end{array}$ \\
\hline 80 & & & & & & & & & & & $\stackrel{0}{\stackrel{ }{0}}$ & $\vec{H}$ & $\stackrel{\vec{t}}{\stackrel{0}{0}}$ & $\stackrel{\vec{t}}{\stackrel{0}{0}}$ & $\begin{array}{l}0 \\
\stackrel{1}{0}\end{array}$ & $\stackrel{m}{\stackrel{m}{0}}$ & $\stackrel{\leftrightarrow}{\leftrightarrow}$ \\
\hline$\vec{b}$ & & & & ڤొ & $\stackrel{\sim}{\tilde{\sigma}}$ & $\stackrel{\text { ڤn }}{0}$ & $\stackrel{\infty}{\stackrel{\infty}{0}}$ & & 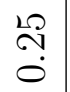 & & $\stackrel{\sim}{\stackrel{\sim}{0}}$ & $\stackrel{10}{\rightleftharpoons}$ & $\stackrel{ }{\sim}$ & $\stackrel{N}{\circ}$ & $\begin{array}{l}0 \\
\stackrel{1}{0}\end{array}$ & $\stackrel{\sim}{\stackrel{\sim}{0}}$ & $\begin{array}{l}\mathscr{0} \\
\stackrel{0}{0}\end{array}$ \\
\hline 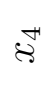 & & & & $\ddot{\sigma}$ & $\ddot{\sigma}$ & $\begin{array}{l}0 \\
0 \\
0\end{array}$ & $\stackrel{R}{0}$ & & $\stackrel{\Gamma}{\dot{0}}$ & & $\begin{array}{l}\infty \\
0 \\
0\end{array}$ & $\begin{array}{l}\overrightarrow{0} \\
\dot{0}\end{array}$ & $\stackrel{H}{0}$ & $\begin{array}{l}0 \\
\bullet \\
0\end{array}$ & $\stackrel{N}{\stackrel{N}{0}}$ & $\begin{array}{l}0 \\
0 \\
0\end{array}$ & $\stackrel{N}{\hat{0}}$ \\
\hline $\overrightarrow{8}$ & & & & $\stackrel{0}{\stackrel{0}{0}}$ & $\stackrel{\overbrace{}}{\stackrel{\overbrace{}}{0}}$ & $\stackrel{0}{\stackrel{0}{0}}$ & $\stackrel{\kappa}{\leftrightarrow}$ & & $\stackrel{1}{\sim}$ & & $\stackrel{H}{\stackrel{H}{0}}$ & 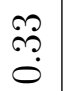 & $\stackrel{\leftrightarrow}{\stackrel{\sim}{0}}$ & 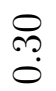 & $\stackrel{1}{\leftrightarrow}$ & $\stackrel{N}{\tilde{O}}$ & $\stackrel{\leftrightarrow}{\dddot{\rho}}$ \\
\hline $0^{\infty}$ & $\stackrel{N}{\stackrel{N}{0}}$ & $\stackrel{\text { Nิ }}{\circ}$ & $\stackrel{\vec{t}}{\stackrel{H}{0}}$ & $\stackrel{0}{\stackrel{0}{1}}$ & $\stackrel{\sim}{\stackrel{1}{0}}$ & $\begin{array}{l}0 \\
\stackrel{0}{0}\end{array}$ & $\stackrel{m}{\stackrel{2}{0}}$ & & $\begin{array}{l}ت \\
\overrightarrow{0}\end{array}$ & & $\stackrel{\stackrel{ }{I}}{\stackrel{0}{0}}$ & $\stackrel{m}{\ddot{0}}$ & $\vec{\prime}$ & $\stackrel{\circ}{\stackrel{1}{0}}$ & $\stackrel{\sim}{\stackrel{1}{0}}$ & $\begin{array}{l}1 \\
0 \\
0\end{array}$ & $\stackrel{\infty}{\stackrel{0}{0}}$ \\
\hline$\stackrel{乛}{8}$ & $\stackrel{\infty}{0}$ & $\begin{array}{l}\infty \\
0 \\
0\end{array}$ & $\begin{array}{l}0 \\
\stackrel{0}{0}\end{array}$ & $\stackrel{\sharp}{\stackrel{H}{0}}$ & $\stackrel{\mathscr{P}}{\stackrel{\leftrightarrow}{0}}$ & 官 & 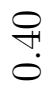 & & $\begin{array}{c}\overrightarrow{10} \\
0\end{array}$ & & $\stackrel{\stackrel{\sim}{\sharp}}{\circ}$ & $\stackrel{\mathscr{m}}{\stackrel{\leftrightarrow}{0}}$ & $\stackrel{\leftrightarrow}{\stackrel{\leftrightarrow}{+}}$ & $\stackrel{0}{0}$ & $\stackrel{N}{\infty}$ & $\stackrel{g}{\stackrel{\leftrightarrow}{0}}$ & $\stackrel{20}{\stackrel{1}{+}}$ \\
\hline$\Re$ & $\stackrel{\infty}{\stackrel{\infty}{\ominus}}$ & ભి & $\begin{array}{l}\infty \\
\stackrel{\leftrightarrow}{0} \\
0\end{array}$ & $\stackrel{\leftrightarrow}{\leftrightarrow}$ & $\begin{array}{l}0 \\
\stackrel{0}{0} \\
0\end{array}$ & $\stackrel{2}{2}$ & $\stackrel{m}{\stackrel{2}{0}}$ & & $\begin{array}{l}m \\
\dddot{m} \\
0\end{array}$ & & $\stackrel{20}{\stackrel{1}{H}}$ & $\begin{array}{l}100 \\
100 \\
0\end{array}$ & $\stackrel{\leftrightarrow}{\stackrel{P}{\leftrightarrow}}$ & $\begin{array}{l}0 \\
\stackrel{0}{0}\end{array}$ & $\stackrel{\curvearrowright}{\ominus}$ & $\overrightarrow{0}$ & $\stackrel{N}{\stackrel{1}{0}}$ \\
\hline$\tilde{b}^{N}$ & $\stackrel{\vec{H}}{\rightleftarrows}$ & $\stackrel{8}{\stackrel{8}{0}}$ & $\stackrel{\infty}{\rightleftarrows}$ & $\stackrel{g}{\stackrel{g}{0}}$ & $\stackrel{\infty}{0}$ & $\stackrel{\infty}{0}$ & $\stackrel{\infty}{0}$ & & $\begin{array}{l}\mathscr{O} \\
\dot{0} \\
0\end{array}$ & & $\stackrel{\infty}{0}$ & $\begin{array}{l}\mathscr{O} \\
\stackrel{0}{0}\end{array}$ & $\stackrel{N}{0}$ & $\stackrel{N}{\circ}$ & $\stackrel{1}{0}$ & $\begin{array}{l}0 \\
\stackrel{-}{0} \\
0\end{array}$ & $\stackrel{\infty}{0}$ \\
\hline \&્తి & $\stackrel{0}{0}$ & $\stackrel{\overbrace{}}{\stackrel{\overbrace{}}{0}}$ & 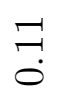 & $\ddot{\infty}$ & $\stackrel{\infty}{\stackrel{\infty}{0}}$ & $\stackrel{\infty}{\stackrel{\infty}{0}}$ & $\stackrel{20}{\stackrel{0}{0}}$ & & $\begin{array}{l}\infty \\
0 \\
0\end{array}$ & & $\stackrel{\overbrace{}}{\stackrel{\Omega}{0}}$ & $\stackrel{N}{ヘ}$ & $\stackrel{\text { }}{0}$ & $\stackrel{\leftrightarrow}{\stackrel{H}{0}}$ & $\stackrel{\overbrace{}}{\Im}$ & $\begin{array}{l}\mathscr{Q} \\
\stackrel{0}{0}\end{array}$ & $\stackrel{10}{\overparen{0}}$ \\
\hline $\mathfrak{S}$ & $\stackrel{\text { P }}{+}$ & $\stackrel{\text { নิ }}{\mathfrak{O}}$ & تִ̣ & $\stackrel{\rightleftarrows}{\not}$ & $\stackrel{\text { ஸे }}{0}$ & 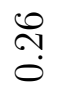 & 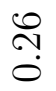 & & $\begin{array}{l}0 \\
\infty \\
0\end{array}$ & & $\begin{array}{l}\infty \\
2 ? \\
0 \\
0\end{array}$ & $\begin{array}{l}\stackrel{0}{*} \\
\stackrel{0}{0}\end{array}$ & $\stackrel{\text { స̦ }}{0}$ & $\stackrel{\stackrel{L}{?}}{\stackrel{0}{0}}$ & \begin{tabular}{l}
$\infty$ \\
$\stackrel{\infty}{0}$ \\
\hdashline
\end{tabular} & $\begin{array}{l}\overrightarrow{0} \\
\dot{0}\end{array}$ & $\begin{array}{l}0 \\
\stackrel{0}{0}\end{array}$ \\
\hline 5 & $\stackrel{\rightleftarrows}{\rightleftarrows}$ & $\stackrel{\sim}{\stackrel{\circ}{0}}$ & $\stackrel{\infty}{\rightleftarrows}$ & $\stackrel{0}{\stackrel{0}{0}}$ & $\stackrel{\circ}{\stackrel{0}{0}}$ & 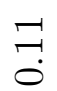 & $\stackrel{7}{\not}$ & & $\underset{+}{\vec{J}}$ & & $\stackrel{\circ}{\stackrel{\circ}{0}}$ & $\begin{array}{l}\mathscr{g} \\
\stackrel{0}{0}\end{array}$ & $\stackrel{\circ}{\stackrel{0}{0}}$ & $\begin{array}{l}\stackrel{g}{0} \\
0\end{array}$ & $\underset{0}{\stackrel{g}{0}}$ & $\vec{F}$ & $\stackrel{8}{\circ}$ \\
\hline $\overrightarrow{8}$ & $\stackrel{10}{\longrightarrow}$ & $\stackrel{\stackrel{\sim}{I}}{\stackrel{0}{0}}$ & $\stackrel{\mathscr{P}}{\stackrel{\leftrightarrow}{0}}$ & $\begin{array}{l}\mathscr{O} \\
\stackrel{0}{0}\end{array}$ & $\begin{array}{l}\stackrel{g}{0} \\
\dot{0}\end{array}$ & $\underset{二}{\rightleftarrows}$ & $\stackrel{\circ}{\stackrel{ }{0}}$ & & $\vec{m}$ & & $\stackrel{\circ}{\stackrel{ }{1}}$ & $\begin{array}{l}\infty \\
0 \\
0\end{array}$ & $\begin{array}{l}0 \\
\stackrel{1}{0}\end{array}$ & $\stackrel{\circ}{\stackrel{1}{0}}$ & $\stackrel{\infty}{0}$ & $\begin{array}{l}\stackrel{\mathfrak{L}}{?} \\
\stackrel{0}{0}\end{array}$ & $\begin{array}{l}0 \\
\ddot{0}\end{array}$ \\
\hline$\vec{\sigma}$ & $\neg$ & $\neg$ & $\neg$ & $\multimap$ & $\neg$ & $\neg$ & $\neg$ & & - & & $\neg$ & - & $\neg$ & $\neg$ & $\neg$ & $\neg$ & $\neg$ \\
\hline 范 & $\stackrel{\stackrel{I}{-}}{\longrightarrow}$ & $\vec{A}$ & $\stackrel{\text { స̆ }}{\stackrel{1}{\sim}}$ & $\underset{\sim}{\stackrel{P}{\longrightarrow}}$ & $\underset{+}{\stackrel{4}{+}}$ & $\begin{array}{c}\stackrel{1}{N} \\
\stackrel{N}{*}\end{array}$ & $\underset{\infty}{\stackrel{H}{S}}$ & $\stackrel{ }{\stackrel{H}{\not}}$ & $\begin{array}{l}\overrightarrow{1} \\
\dot{P} \\
\dot{P}\end{array}$ & $\begin{array}{l}\grave{N} \\
\text { ড̀ } \\
\text { N }\end{array}$ & $\underset{\sim}{\ddot{P}}$ & $\underset{亡}{\stackrel{\rightleftarrows}{\rightleftarrows}}$ & 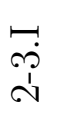 & 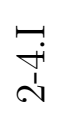 & $\underset{\infty}{\stackrel{T}{\sim}}$ & 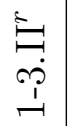 & 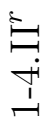 \\
\hline
\end{tabular}




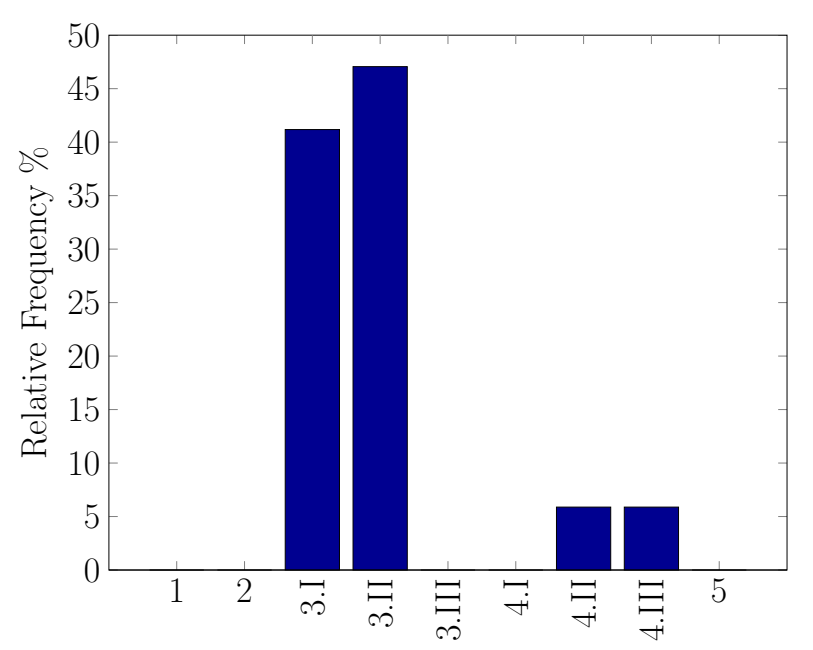

(a) Midships

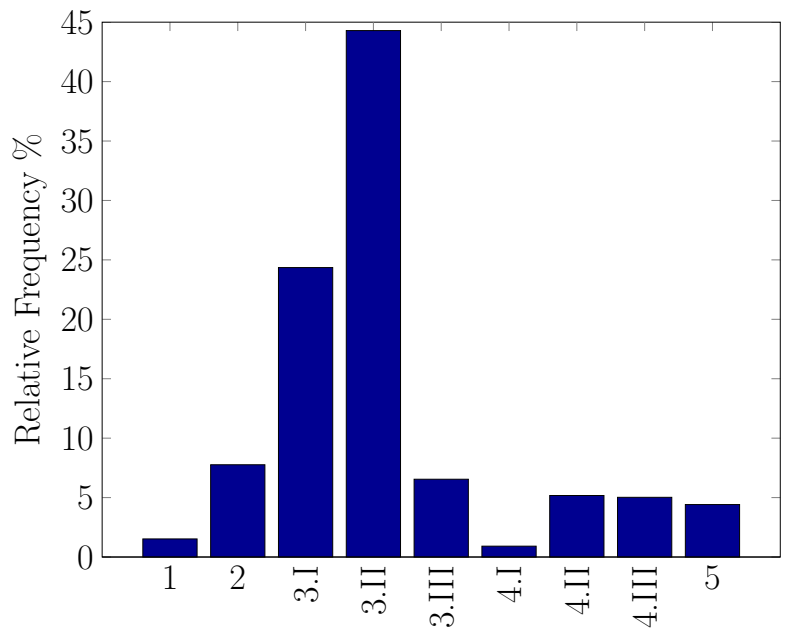

(b) Bow

Figure 2.16: Day 1 profile distributions.

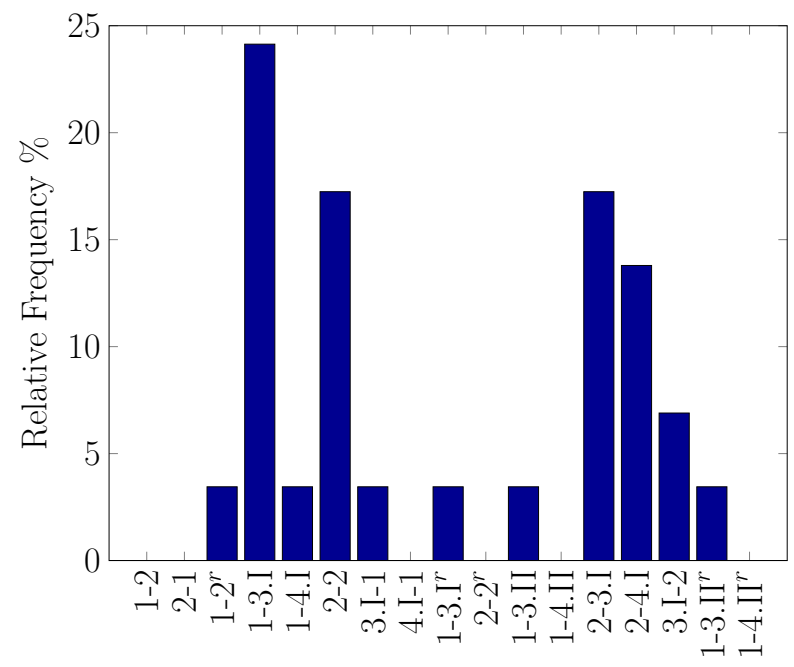

Figure 2.17: Distribution of Type 5 profiles for Day 1 - bow. 


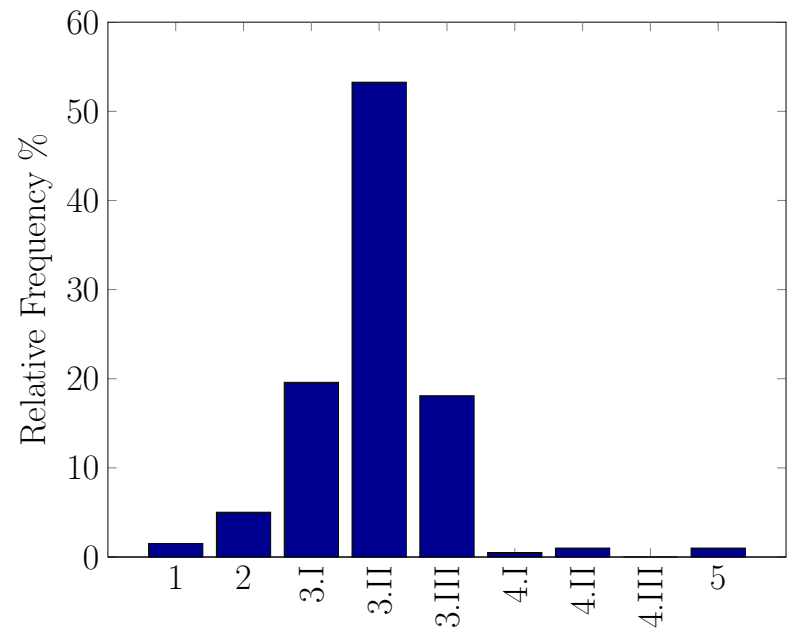

(a) Midships

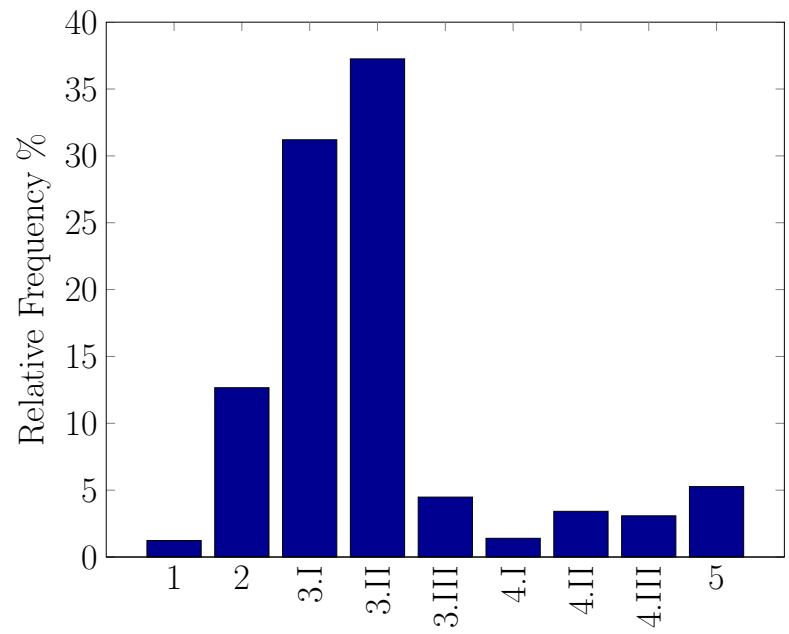

(b) Bow

Figure 2.18: Day 2 profile distributions.

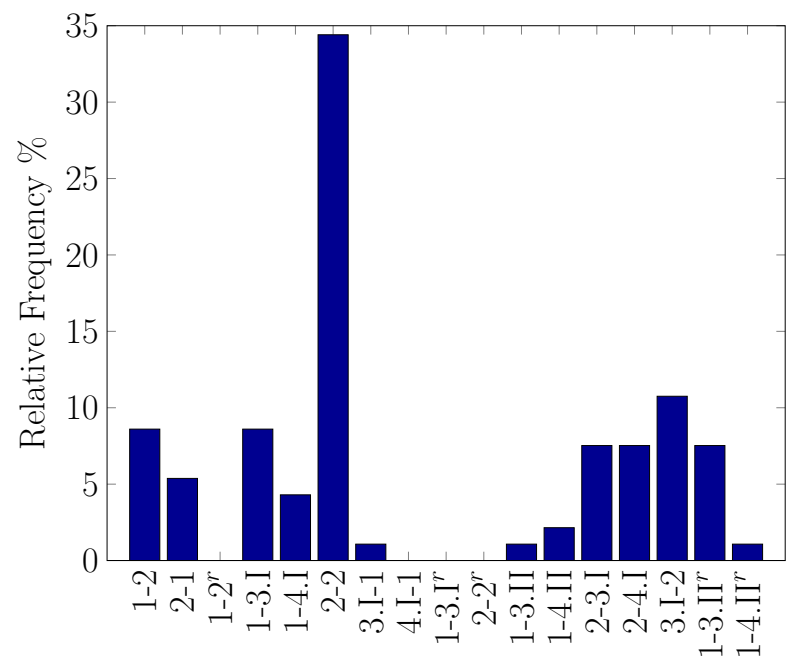

Figure 2.19: Distribution of Type 5 profiles for Day 2 - bow. 


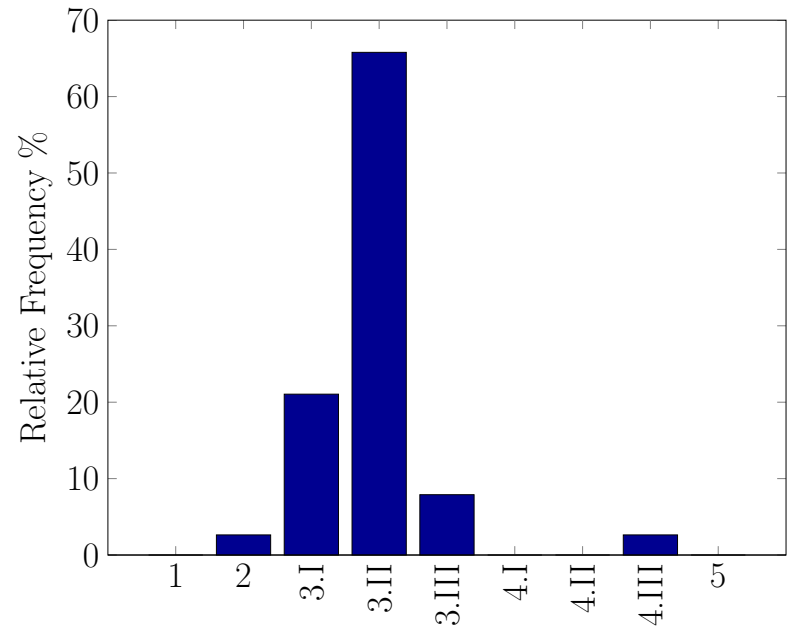

(a) Midships

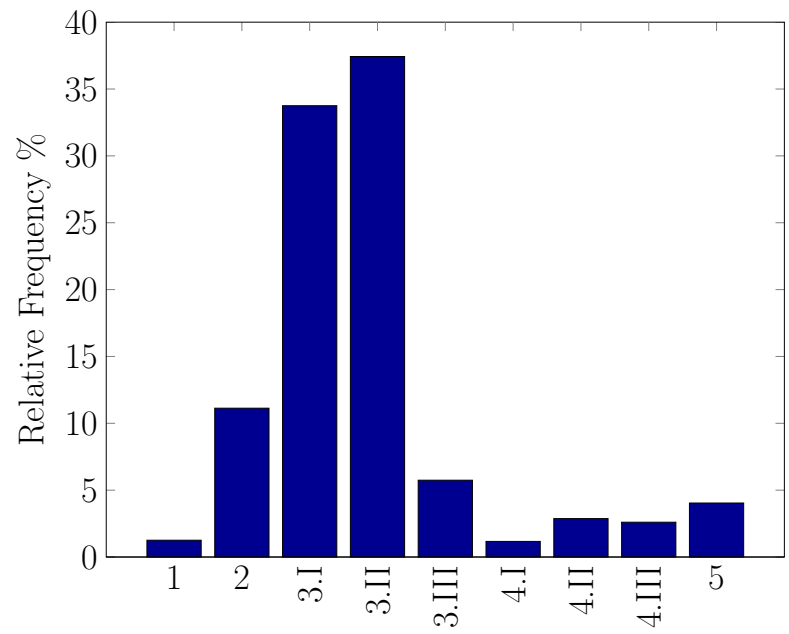

(b) Bow

Figure 2.20: Day 3 profile distributions.

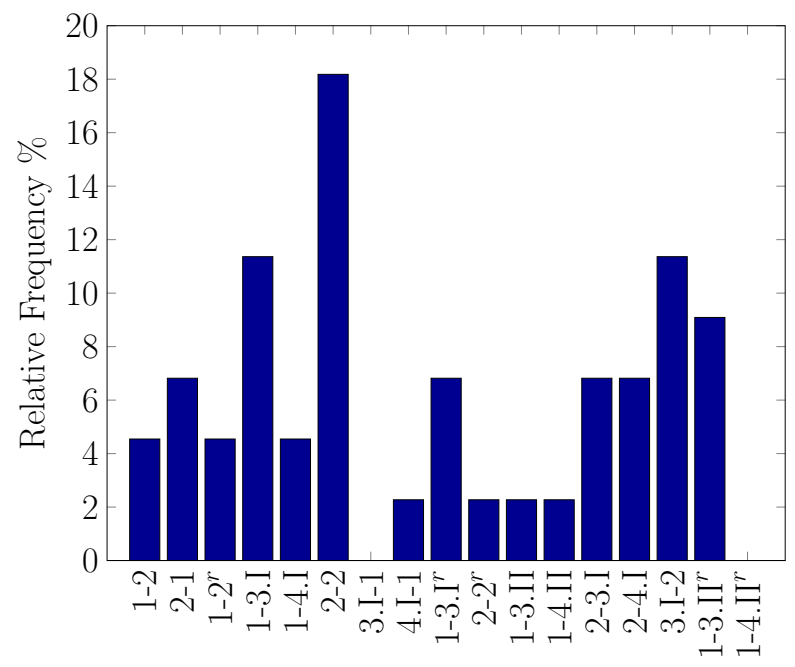

Figure 2.21: Distribution of Type 5 profiles for Day 3 - bow. 


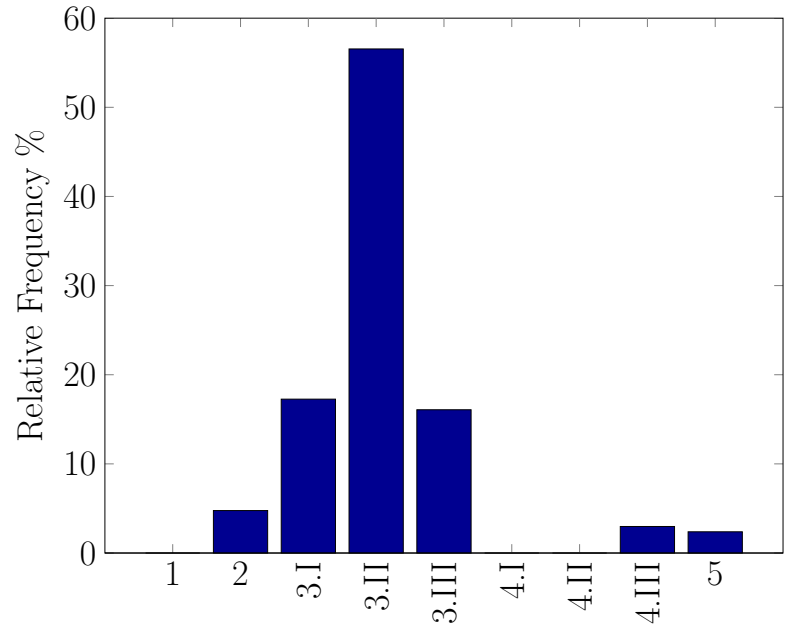

(a) Midships

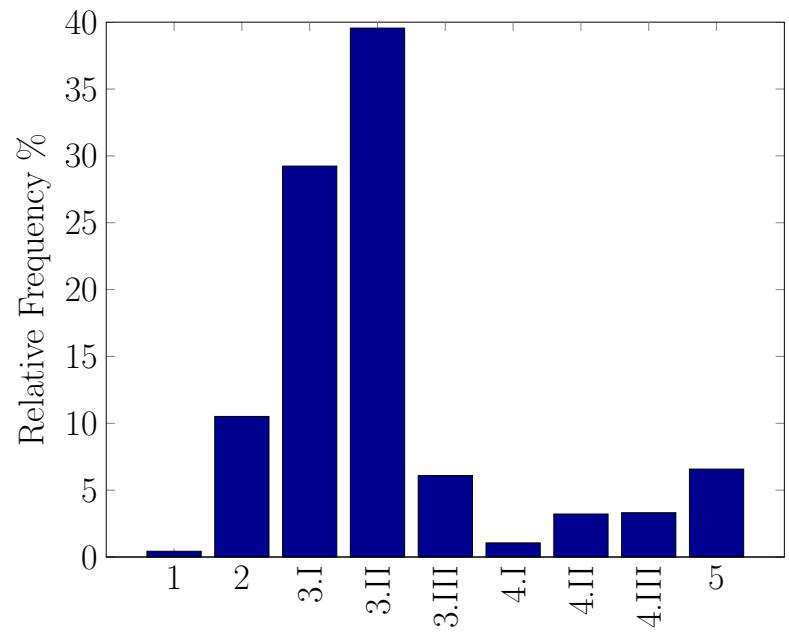

(b) Bow

Figure 2.22: Day 4 profile distributions.

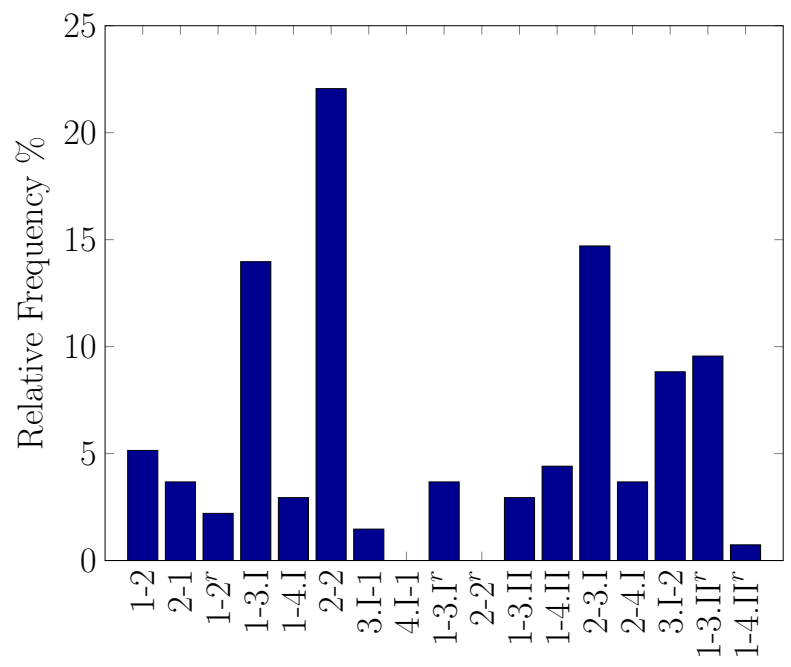

Figure 2.23: Distribution of Type 5 profiles for Day 4 - bow. 


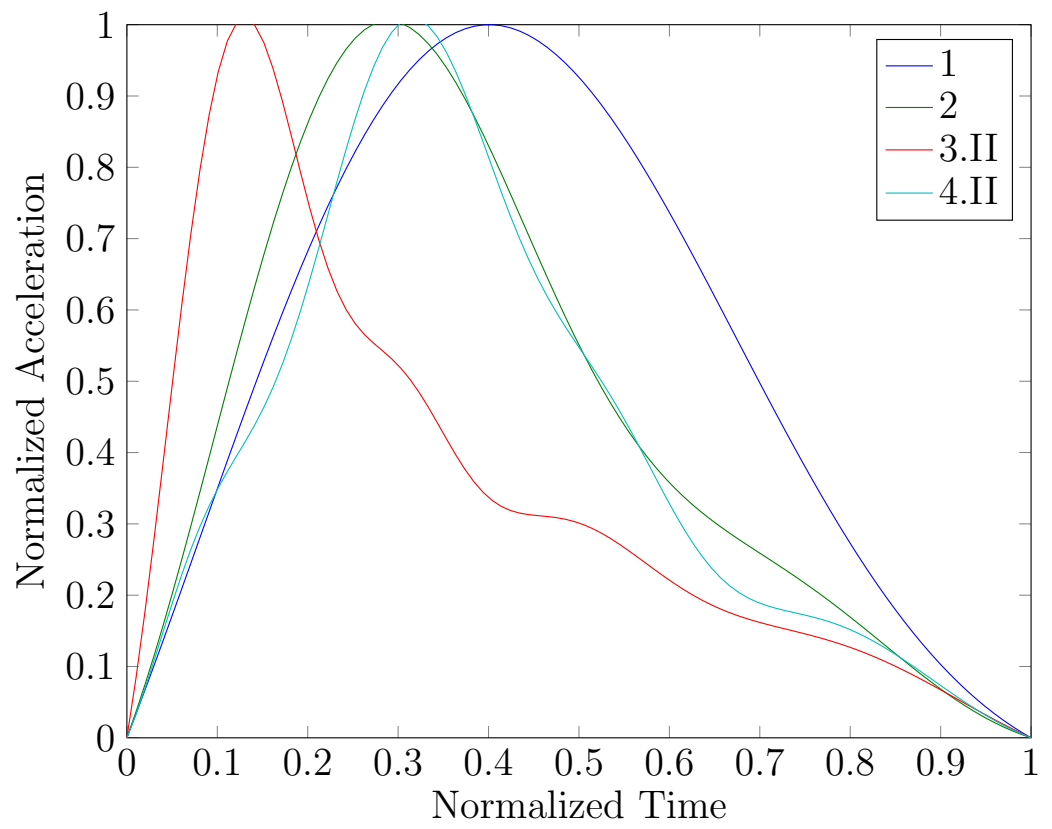

Figure 2.24: Profiles at parameter mean values of Day 1 at the bow. 


\subsection{Discussion}

In the first part of this discussion comments are made on the curve fitting process. In the second part, the results are discussed.

\subsubsection{On the Curve Fitting Process}

The peak detection rate varies between $83 \%$ and $100 \%$ which shows that the chosen equation form fits the observed acceleration pulses fairly well. Inspection of undetected pulses shows that for the majority of these pulses a different set of initial parameters or parameter constraints (which are input to the curve fitting algorithm) can lead to a solution. Other curves, however, have shapes which could not be captured with a sum of Gaussian curves.

The amplitude of the first Gaussian curve in the sum was constrained to be equal to approximately 1 such that its peak coincides (approximately) with the peak of the pulse. The reason why it cannot be set to exactly 1 is that the equation terms cannot be equal to 0 on $] 0,1[$. It is possible to obtain a fit in which the underlying curves overlap at the peak time. However, this overlapping is not favourable because the peak time would not appear "naturally" in the equation.

It was decided to work with the amplitudes of the Gaussian curves $a_{n}$ rather than the corresponding pulse amplitudes $h_{n}$. Since $h_{n}$ are close to but not exactly equal to amplitudes at characteristic points (e.g., inflection points), it was decided to reduce the number of computations by working with $a_{n}$ instead. However, as it was shown in Section 2.2.2, $a_{n}$ can be expressed in terms of $h_{n}$. 


\subsubsection{On the Results}

The profile distribution for Types 1 through 4 for each of the four days is almost identical which is not surprising since the variations in wave height and period were small during the course of the sea trial (Figure 2.1). Some profiles are not present in the time histories collected at the centre portion of the boat which indicate that the characteristics of the motion at the midships portion of the boat is different than at the bow. This is expected since the motion of the bow is more pronounced than the midships portion of the boat. As shown in Table 2.2, the midships portion experiences much fewer impacts than the bow of the boat.

More variability is observed in the profile distributions of Type 5 profiles. This is expected since only a small portion of pulses are of Type 5. For the majority of the Type 5 profiles, the acceleration peak value occurs during the first pulse. Overall, the most common variation is Type 2-2 and the least common variations are Type

\section{I-1 and 4.I-1.}

Type 5 profiles were classified differently than the other types because they consist of a combination of profiles. As a consequence, a classification according to the number of underlying Gaussian curves is not appropriate. For instance, in a combination of 4 Gaussian term, the third Gaussian curve could correspond to either the third shoulder of the first pulse or the second highest peak or the first shoulder of the second pulse. Therefore, the distribution of the equation parameters would be multimodal and the mean values of the parameters would not be an adequate description. Subdividing Type 5 variations into the categories shown in the previous section resolves this issue.

When comparing the parameter mean values of each type (1-4) for different days it 
can be seen that the values are close to each other. This suggests that the pulse shapes are similar in the analyzed time histories. The duration and maximum accelerations of the pulses differ, however. It can be seen that the accelerations increase with increasing wave height. While pulse durations for each type are within the same range, it seems that there is no correlation between pulse duration, wave height, and period. Types 3.I and 3.II were shown to have consistently the highest accelerations. These profiles are also the most frequent covering more than $60 \%$ of detected pulses. The parameter mean values for Type 5 are within the same range when comparing different days. However, once again the small sample size of Type 5 profiles results in values wich are more variable than for Types 1 through 4 .

\subsection{Conclusion}

The observed acceleration pulses were classified and characterized. The curve fitting equations and algorithm that were developed are reliable as evidenced by the high detection rate. The different profiles were characterized by their parameter mean values. These values suggest a close similarity between the pulse shapes observed in different sea conditions. In the next chapter, the distributions of the parameters are exploited in order to generate time histories with the same statistical characteristics as the ones from the sea trial data. 


\section{Chapter 3}

\section{Synthetic Time History Generation}

The generation of synthetic acceleration time histories consisting of the profiles seen in Chapter 2 requires knowledge of the distributions of the equation parameters and the dependencies between these parameters. Formally, given an m-by-n matrix $\mathrm{X}=$ $\left[X_{1} X_{2} \ldots X_{n}\right]$ where $\mathrm{m}$ is the number of observations and $\mathrm{n}$ is the number of variables, it is required to find an l-by-n matrix $\mathrm{Y}$ whose entries are not identical to the entries in $\mathrm{X}$, and such that $Y_{i}$ has the same distribution as $X_{i}$ and the inter-dependencies between the $Y_{i}$ 's are the same as those between the $X_{i}$ 's. In here, a matrix $\mathrm{X}$ is associated with each type of pulse and is obtained by curve fitting the original time histories. The objective behind simulating equation parameters is to extend a given time history or to synthesize a new one. Put differently, the simulation procedure serves as a way to interpolate between the original parameter values.

Two methods are presented in this chapter for simulating dependent random variables. The first method is based on the theory of mathematical functions called copulas. The second method consists of obtaining a non-parameteric estimate of the joint probability density function of $\mathrm{X}$ and using it to generate synthetic data. These 
two methods were used to generate synthetic pulses.

In order to create transitions between impacts, cam profiles were fitted in between consecutive pulses. The properties of these profiles were adjusted so as to minimize drift in both velocity and displacement. The two methods are presented in Section 3.1. Section 3.2 presents the inter-pulse characteristics. Simulation results are presented in Section 3.3. The results are discussed in Section 3.4. Concluding remarks are made in Section 3.5.

\subsection{Synthetic Pulse Generation Methods}

The procedure for simulating dependent random variables using copulas is outlined in the first part of this section. The method for estimating the joint probability density of the variables and its application to data simulation is presented in the second part.

\subsubsection{Simulating Dependent Random Variables using Cop- ulas}

A copula is a function which joins a multivariate distribution function to its one-dimensional marginal distribution functions [14]. This is formally stated by Sklar's Theorem: Let $H$ be a two-dimensional distribution function with marginal distribution functions $F_{1}$ and $F_{2}$. Then there exists a copula $C$ such that $H\left(x_{1}, x_{2}\right)=C\left(F_{1}\left(x_{1}\right), F_{2}\left(x_{2}\right)\right)$. Conversely, for any distribution functions $F_{1}$ and $F_{2}$ and any copula $C$, the function $H$ defined above is a two-dimensional distribution function with marginals $F_{1}$ and $F_{2}$. Furthermore, if $F_{1}$ and $F_{2}$ are continuous, $C$ is 
unique.

The terms $F_{1}, F_{2}$, and $H$ denote cumulative probability distributions, e.g., $\mathrm{F}\left(x_{1}\right)$ $=\mathrm{P}\left(X_{1} \leq x_{1}\right)$. The copula $\mathrm{C}$ is the joint distribution function for the random variables $U_{1}=F_{1}\left(X_{1}\right)$ and $U_{2}=F_{2}\left(X_{2}\right)$. Note that $\mathrm{F}\left(X_{1}\right)$ and $\mathrm{G}\left(X_{2}\right)$ are uniformly distributed on $[0,1]$. In essence, a copula contains the dependence structure information of a data set $\mathrm{X}=\left[\begin{array}{lll}X_{1} & X_{2} \ldots X_{n}\end{array}\right]$. Knowing the copula of a data set $\mathrm{X}$ and the individual marginals $F_{i}$, it is then possible to simulate a similar data set $\mathrm{Y}$ in a two step procedure [15]. Random variables $\mathrm{U}=\left[U_{1} U_{2} \ldots U_{n}\right]$ are first drawn from the distribution C. A tranformation is applied to each $U_{i}$ in order to obtain simulated variables $Y_{i}$. From probability theory, if $X_{1}$ is a continous random variable with distribution function $F_{1}$, then the random variable $U_{1}=F_{1}\left(X_{1}\right)$ has a uniform distribution on $[0,1]$. Conversely, applying the inverse transformation of a distribution $F_{1}$ to a uniformly distributed random variable $U_{1}$ results in a random variable with the distribution $F_{1}$. Therefore $Y_{i}=F_{i}^{-1}\left(U_{i}\right)$.

While the simulation procedure is straightforward, it remains to find $\mathrm{C}$ and all the $F_{i}$. Constructing the empirical copula of a given data set is possible [16]. However, it was found that the Gaussian copula is an adequte model for the depencence structure of the observed data. A Gaussian copula is parameterized by a correlation matrix. A suitable parameteric model (e.g., normal, beta, lognormal) for the individual variables $X_{i}$ was not found. For this reason, the empirical distribution of each variable was used instead. The complete simulation procedure for each type of pulse can be summarized by the following steps:

1. Compute the correlation matrix of X; 
2. Draw random numbers $U$ from the Gaussian copula with correlation matrix computed from Step 1;

3. Determine the empirical distribution of each variable $X_{i}$; and

4. Invert each $U_{i}$ to obtain the simulated variables $\mathrm{Y}$.

This procedure is repeated for each type of pulse. The matrix $\mathrm{X}$ is obtained from the curve fitting process described in Chapter 2. The simulated parameters in $\mathrm{Y}$ are then plugged into the representative equations in order to obtain synthetic pulses.

\subsubsection{Simulating Dependent Random Variables by Non- parametric Density Estimation}

The probability density function $f$ of a random vector $X_{i}$ is a function that describes the distribution of $X_{i}$ and allows for calculating probabilities associated with $\mathrm{R}$ :

$$
P\left(a<X_{i}<b\right)=\int_{a}^{b} f(x) \mathrm{dx} \text { for all } \mathrm{a}<\mathrm{b}
$$

Density estimation is the construction of an estimate of $f$ from observed data [17]. The most basic estimator is the histogram. For $\mathrm{X}=\left[\begin{array}{ll}X_{1} & X_{2} \ldots X_{n}\end{array}\right]$ with $\mathrm{m}$ observations, the variable's space is subdivided into hyper-rectangles with size $h_{1} \times h_{2} \times \ldots \times h_{n}$ (Figure 3.1). The joint probability density function at a point with coordinates $\mathbf{x}$ in the hyper-rectangle $B_{k}$, is then estimated as [18]:

$$
\hat{f}_{\text {Hist }}(\mathbf{x})=\frac{\# \text { of data points in } \mathrm{B}_{\mathrm{k}}}{m h_{1} h_{2} \ldots h_{n}} ; \mathbf{x} \text { in } \mathrm{B}_{\mathrm{k}}
$$


The probability that a point falls into $B_{k}$ is then:

$$
P\left(\mathbf{x} \in B_{k}\right)=\frac{\# \text { of data points in } \mathrm{B}_{\mathrm{k}}}{m}
$$

When the data is normally distributed, the optimal bin size is given by Scott's normal reference rule [18]:

$$
h_{i} \approx 3.5 \sigma_{i} m^{\frac{-1}{2+n}} ; i=1, \ldots, n
$$

where $\sigma_{i}$ is the standard deviation of the $i^{\text {th }}$ variable, $m$ is the number of observations, and $n$ is the number of variables.

However, this rule yields large $h_{i}$ for skewed data, which is the case with the parameters at hand. Therefore, the normal reference rule was used as a starting point for determining bin size. Fine tuning of $h_{i}$ was performed iteratively and by visual inspection. The following estimate was ultimately used:

$$
h_{i}=\frac{\max \left(\mathrm{X}_{\mathrm{i}}\right)-\min \left(\mathrm{X}_{\mathrm{i}}\right)}{15} ; i=1, \ldots, n
$$

The probability associated with each hyper-rectangle is then input into a random number generator to produce a synthetic data set Y. Synthetic pulses are then produced by inputting the parameters in $\mathrm{Y}$ into the representative equations as described in the previous section. 


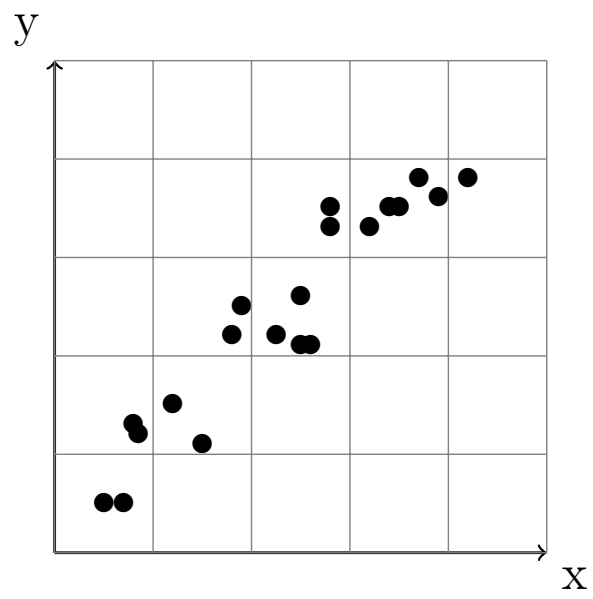

Figure 3.1: Illustration of space discretization for two variables.

\subsection{Inter-Pulse Characteristics}

Synthetic acceleration pulses were generated using the methods described in Sections 3.1.1 and 3.1.2. Cam profiles were fitted in between consecutive pulses:

$y_{\text {cam }}(x)= \begin{cases}k_{1} \sin \left(\frac{\pi x}{2 t_{1}}\right) & 0 \leq x<t_{1}, \\ k_{1} & t_{1} \leq x<t_{1}+t_{2}, \\ \left(1-k_{2}\right)\left(\sin \left(\frac{\pi\left(x+\frac{t_{3}}{2}-t_{1}-t_{2}\right)}{t_{3}}\right)-1\right)-k_{1} & t_{1}+t_{2} \leq x<t_{1}+t_{2}+t_{3}, \\ \cos \left(\frac{\pi\left(x-t_{1}-t_{2}-t_{3}\right)}{2 t_{4}}\right) & t_{1}+t_{2}+t_{3} \leq x \leq t_{1}+t_{2}+t_{3}+t_{4} .\end{cases}$

where $k_{1}$ is the amplitude of the first portion of the profile, $k_{2}+k_{1}=-1$ which is the total amplitude, and $t_{1}+t_{2}+t_{3}+t_{4}$ is the total duration.

Figure 3.2 shows an example of a motion cycle. The parameters of the cam profiles were adjusted at each cycle such as to ensure continuous jerk and to correct drift in both velocity and displacement. To ensure compatibility of slopes between the ends of a cam profile and its two adjoining pulses, parameters $t_{1}$ and $t_{4}$ were written as 
follows:

$$
\begin{aligned}
\frac{\mathrm{d}\left(k_{1} \sin \left(\frac{\pi x}{2 t_{1}}\right)\right)}{\mathrm{d} x}= & s_{1} \text { for } x=0, \text { and } \frac{\mathrm{d}\left(\cos \left(\frac{\pi\left(x-t_{1}-t_{2}-t_{3}\right)}{2 t_{4}}\right)\right)}{\mathrm{d} x}=s_{2} \text { for } x=t_{1}+t_{2}+t_{3}+t_{4} \\
& \Rightarrow t_{1}=\frac{k_{1} \pi}{2 s_{1}}, \text { and } t_{4}=-\frac{\pi}{2 s_{2}}
\end{aligned}
$$

where $s_{1}$ is the jerk at the end of the impact portion of a cycle and $s_{2}$ is the jerk at the begining of the following cycle.

It was assumed that following the impact part of the cycle, the displacement should return as close as possible to its value at the begining of the cycle. Therefore, the target velocity at the begining of any cycle should satify:

$$
\begin{aligned}
\int_{\text {impact }}(v+c) \mathrm{dt} & =\Delta d+c \mathrm{~T}=0 \\
\Rightarrow c & =-\frac{\Delta d}{T}
\end{aligned}
$$

where $v$ is the velocity, $d$ is the displacement, $\mathrm{T}$ is the duration of the impact portion of the cycle, and $c$ is the corrective offset that needs to be added to $v$ at the impact portion of a cycle.

The transition part of a cycle should return the displacement as close as possible to its baseline which was arbitrarily chosen as zero. An additional constraint was imposed that the displacement should be near the baseline at $t_{1}+t_{2}$, which is before the boat, or portion of it, starts to free fall. This constraint is required in order to pull up the displacement if it starts to drift downwards (Figure 3.3). In summary, 
the parameters of a cam profile, $k_{1}, k_{2}$, and $t_{2}$, should minimize:

$$
\begin{aligned}
& \delta_{1}=d_{\text {end of cycle }} \\
& \delta_{2}=v_{\text {end of cycle }}-v_{\text {target }} \\
& \delta_{3}=d_{t_{1}+t_{2}}
\end{aligned}
$$

The acceleration pulses at hand correspond to wave heights of up to 2 metres (Chapter 2). Based on this wave height, a threshold of \pm 0.5 metres was chosen of $\delta_{1}$ and $\delta_{3}$, and a threshold of $0.5 \mathrm{~m} / \mathrm{s}$ was chosen for $\delta_{2}$. If at any given cycle, the solution for the parameters of $y_{\text {cam }}$ results in $\delta$ higher than the prescribed limits, then a different cam profile is used:

$$
y_{\text {cam } 2}(x)= \begin{cases}k \sin \left(\frac{\pi x}{2 t_{1}}\right) & 0 \leq x<t_{1}, \\ k & t_{1} \leq x<t_{1}+t_{2}, \\ k \cos \left(\frac{\pi\left(x-t_{1}-t_{2}\right)}{2 t_{3}}\right) & t_{1}+t_{2} \leq x \leq t_{1}+t_{2}+t_{3} .\end{cases}
$$

where $k$ is the amplitude and $t_{1}+t_{2}+t_{3}$ is the total duration of the profile.

Similarly to the first profile, the parameters $t_{1}$ and $t_{2}$ are expressed in terms of $s_{1}$ and $s_{2}$ :

$$
t_{1}=\frac{k \pi}{2 s_{1}}, \text { and } t_{4}=-\frac{k \pi}{2 s_{2}}
$$

In some cases, the required change in velocity is lower than what can be achieved with $y_{\text {cam }}$. In other cases, a larger downwards acceleration is required. For these reasons, the amplitude $k$ for $y_{\text {cam } 2}$ was not constrained as opposed to the other profile which has a fixed amplitude of -1 . The two unknowns of $y_{\text {cam } 2}$, which are 
$k$ and $t_{2}$, are solved for so as to minimize $\delta_{1}$ and $\delta_{2}$. In the case where suitable parameters are not found, then the errors are corrected at the next cycle by the first cam profile, as shown in Figure 3.3.

The representative equation of each type was integrated analytically using the Symbolic Toolbox in Matlab. The output expression for Type 1 can be found in Appendix E. A non-linear equation solver (fsolve in Matlab) was used for each cycle to determine the parameters of the cam profiles. 

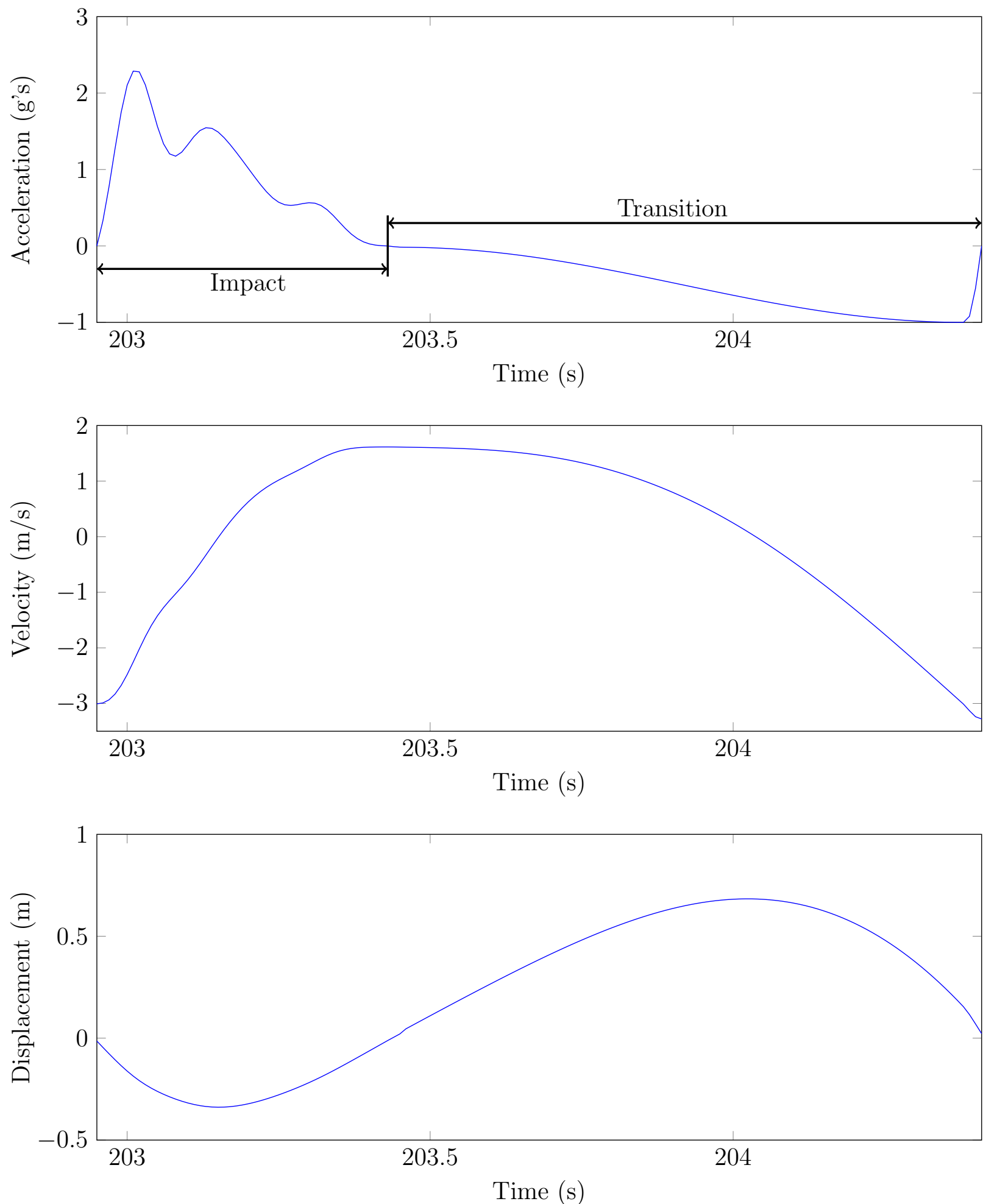

Figure 3.2: Example of a motion cycle. 

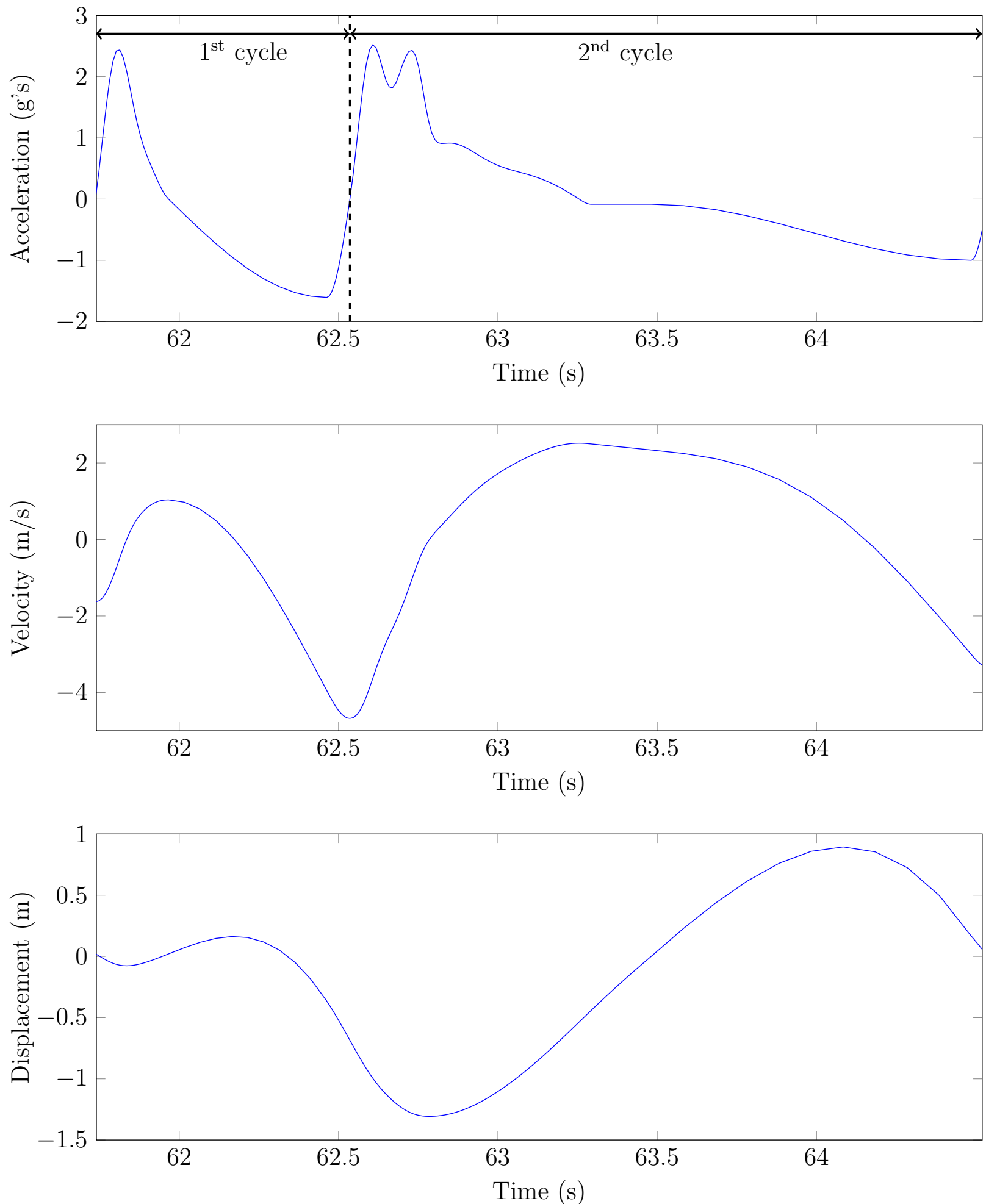

Figure 3.3: Example of drift correction. 


\subsection{Results}

A syntethic time history consisting of 1785 pulses was generated using the pulse characteristics of the Day 2-forward position time hisory (Figure 3.4). Type 2 pulses were chosen to demonstrate the pulse simulation methods. Figures 3.5 and 3.6 show the relationships between the parameters of the Type 2 profile as compared with the simulated parameters by both the copula and density estimation methods. Further, the individual parameter distributions of the original parameters and the simulated ones are shown in Figures 3.7 through 3.9. The generated pulses were fed back into the curve fitting algorithm. The detection rate was found to be $95 \%$. The parameter mean values are compared in Tables 3.1 through 3.4. Finally, Table 3.5 shows a comparison between the number of types prescribed by the random number generator and the number of detected pulses by the curve fitting algorithm. 

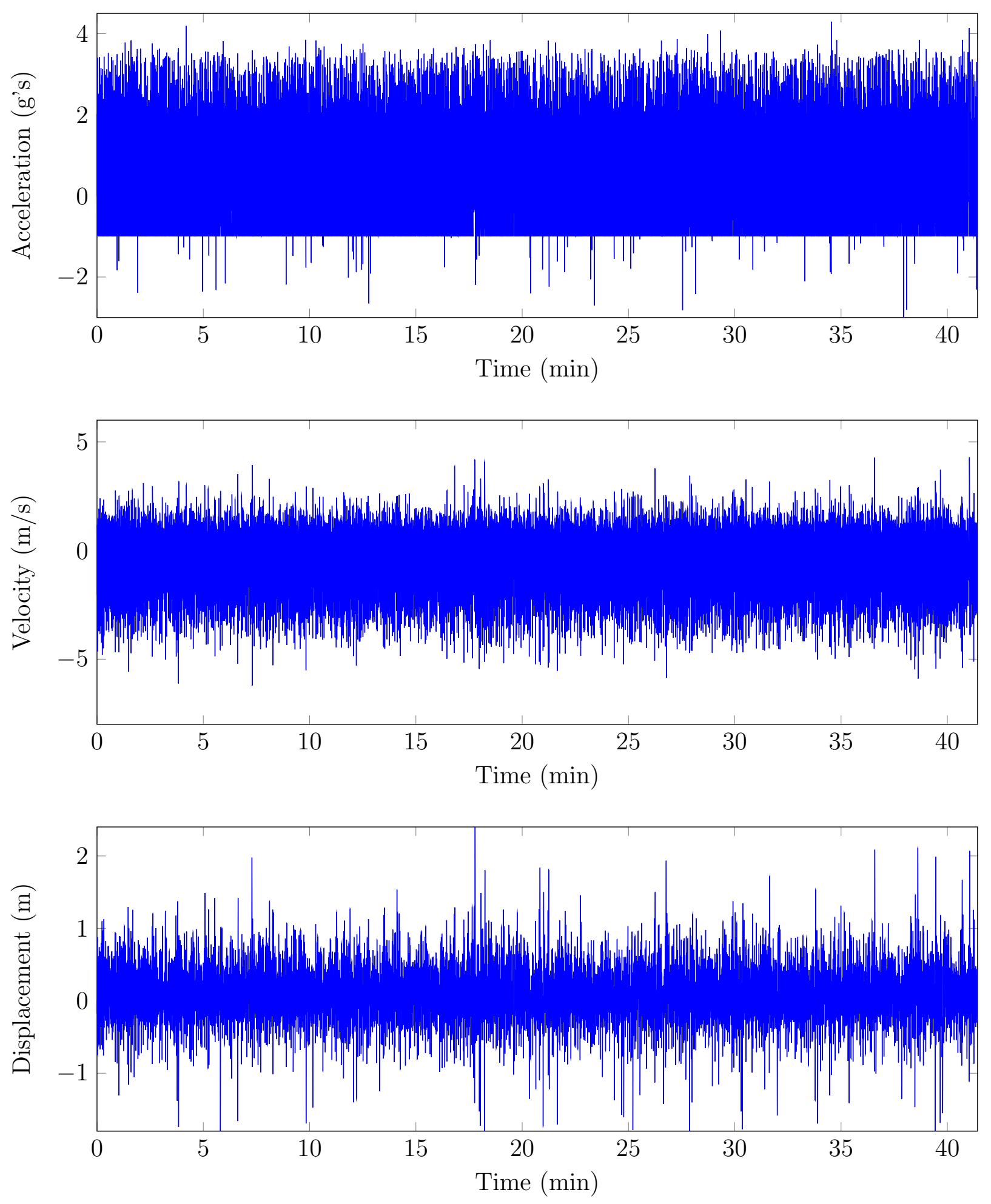

Figure 3.4: Synthetic time history. 


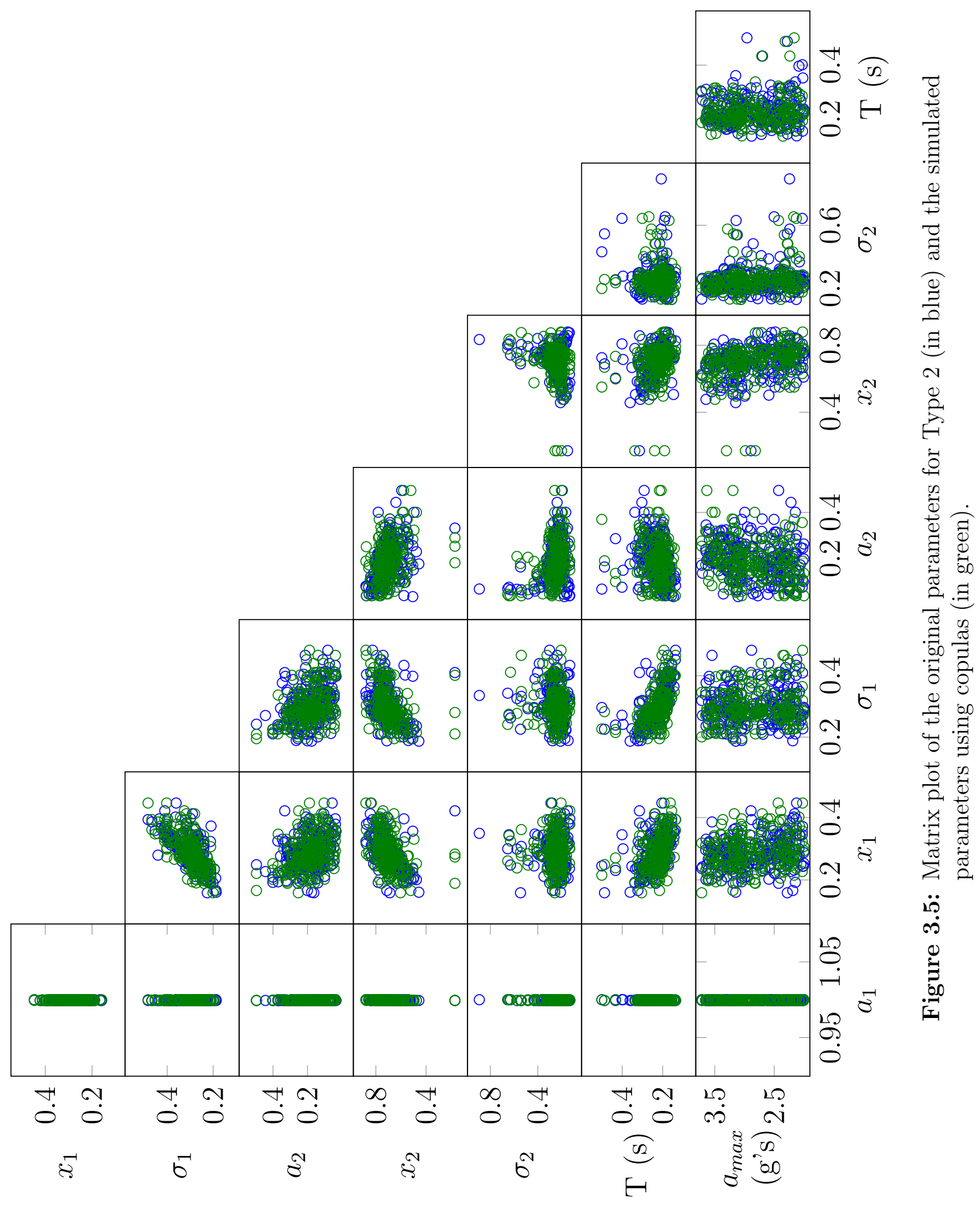




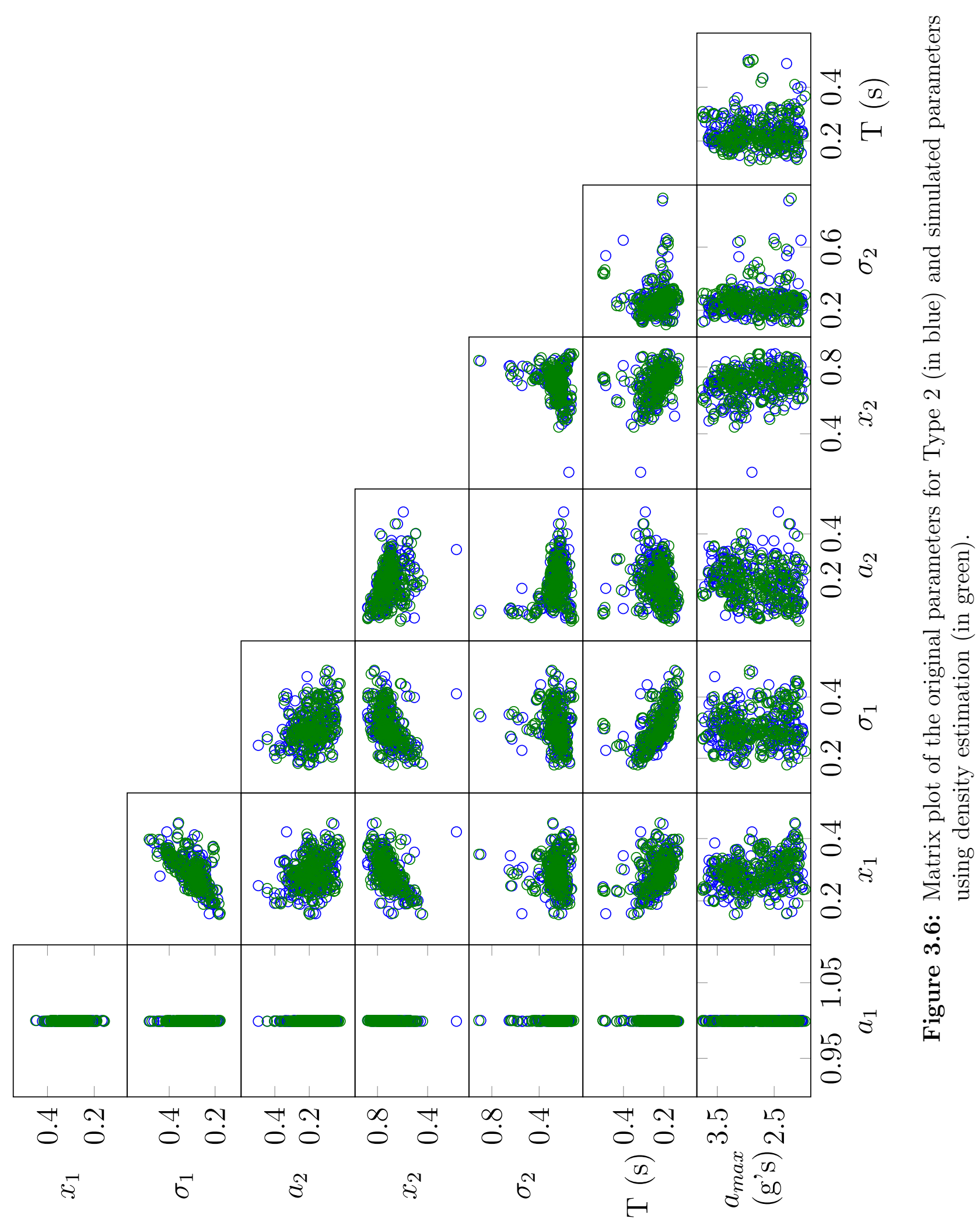



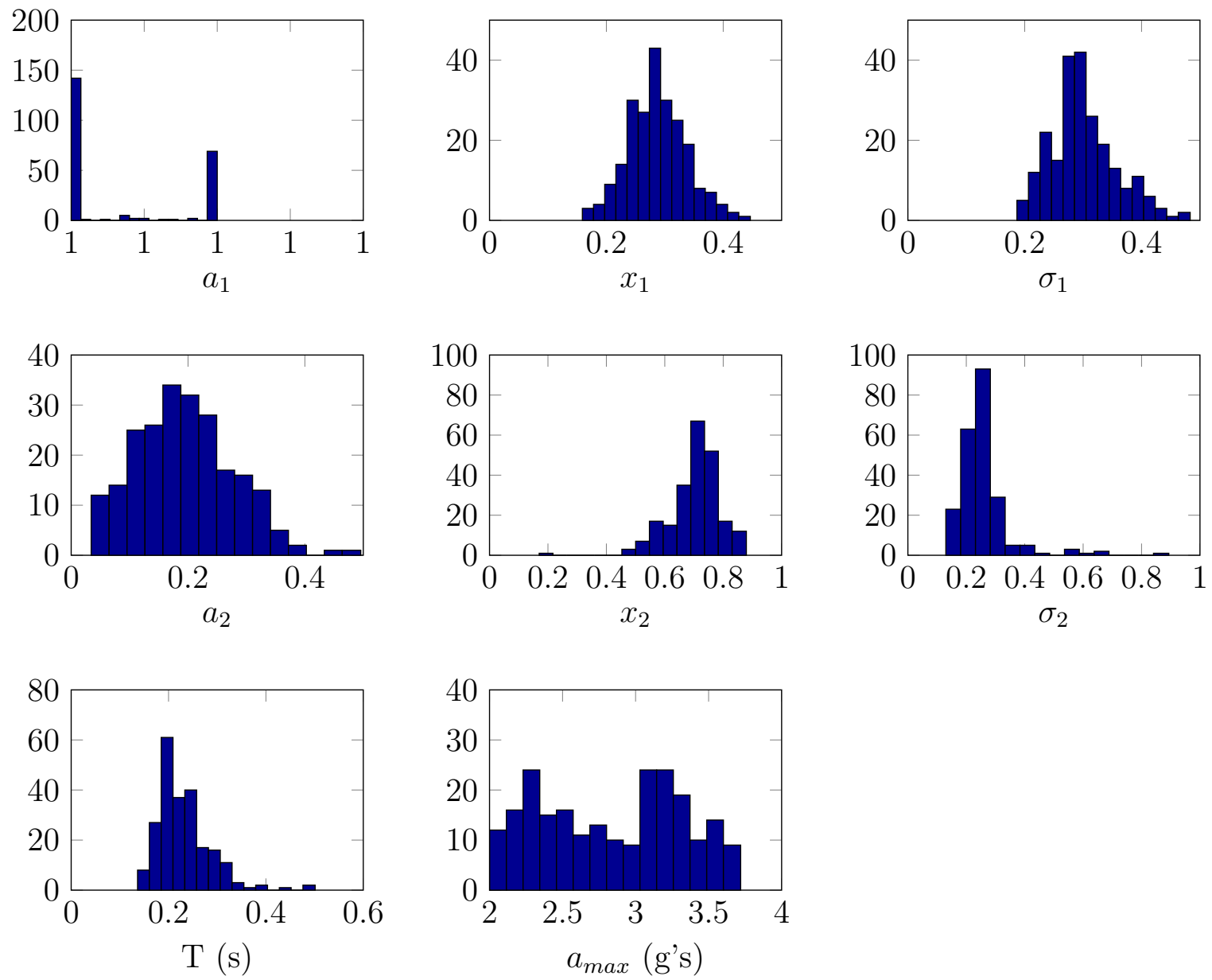

Figure 3.7: Distribution of the original parameters for Type 2. 

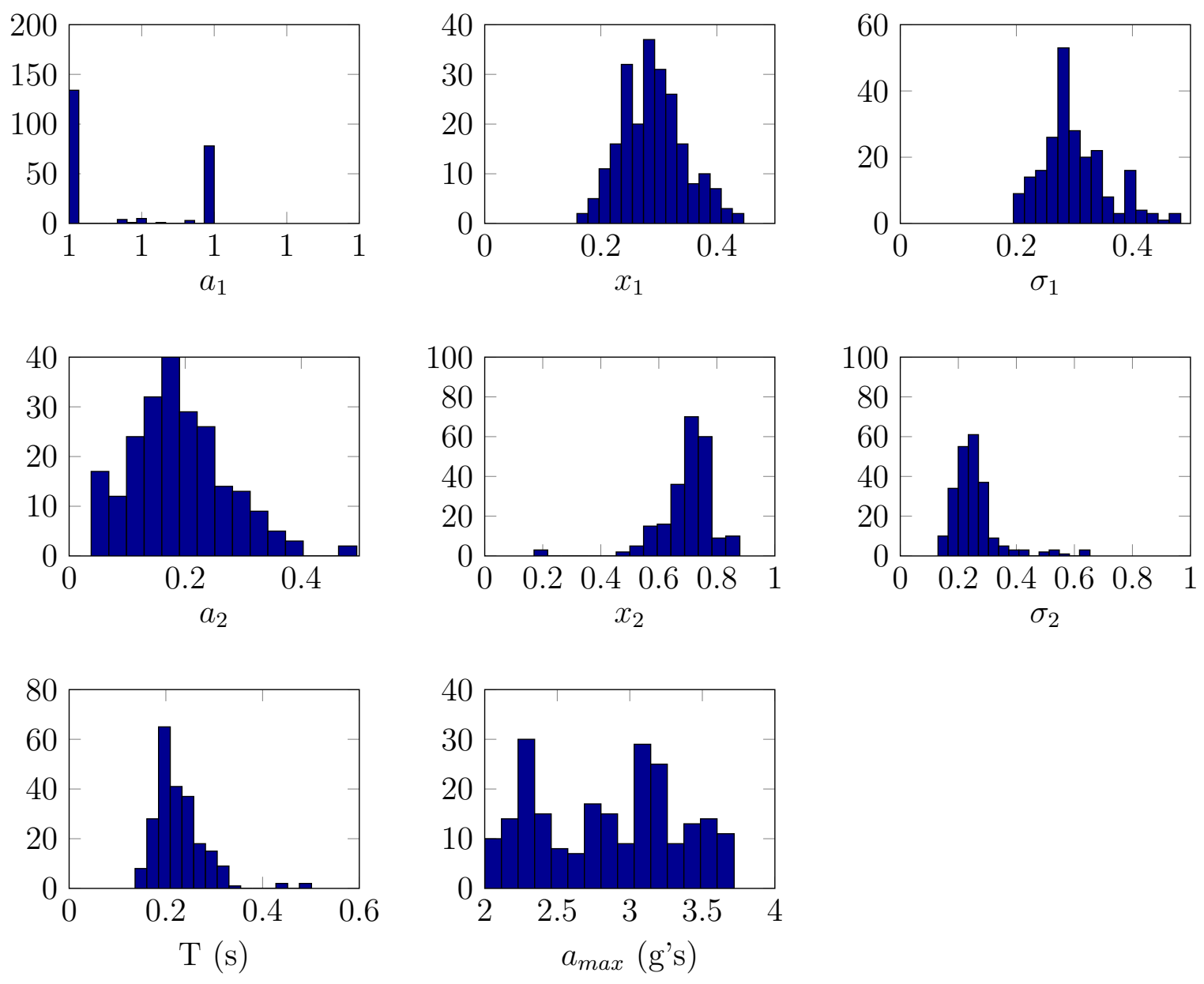

Figure 3.8: Distribution of the simulated parameters using copulas for Type 2. 

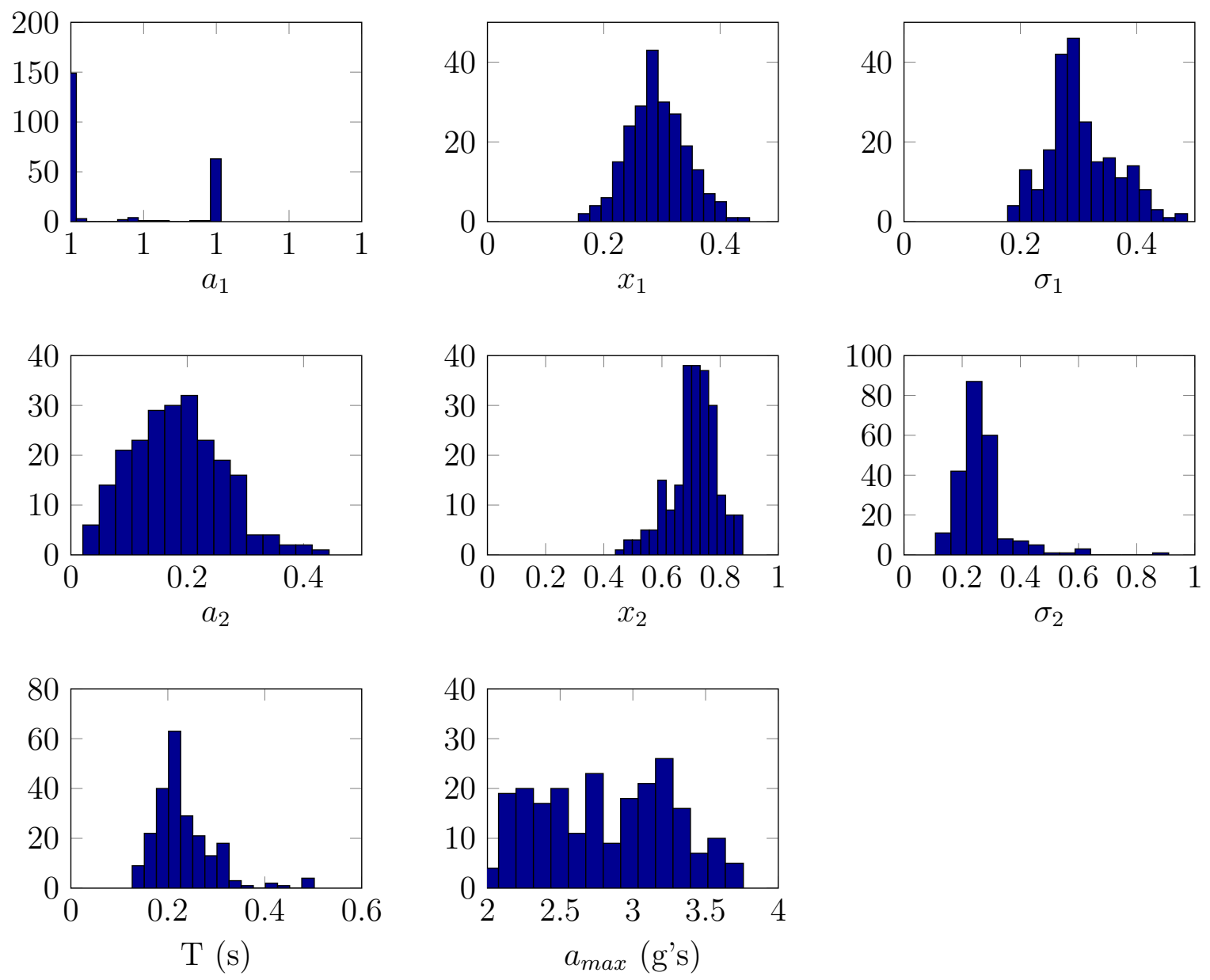

Figure 3.9: Distribution of the simulated parameters using density estimation for Type 2. 


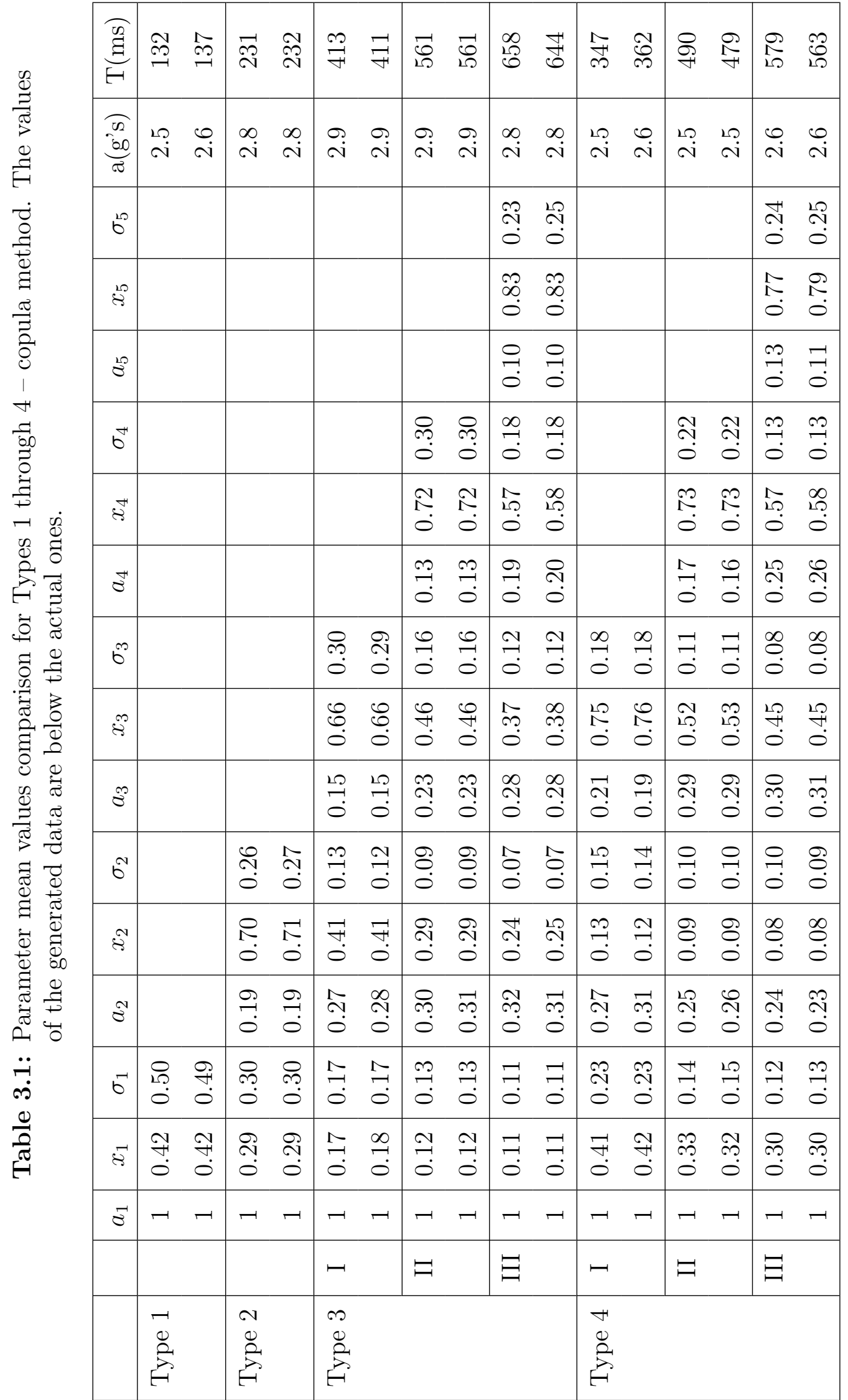




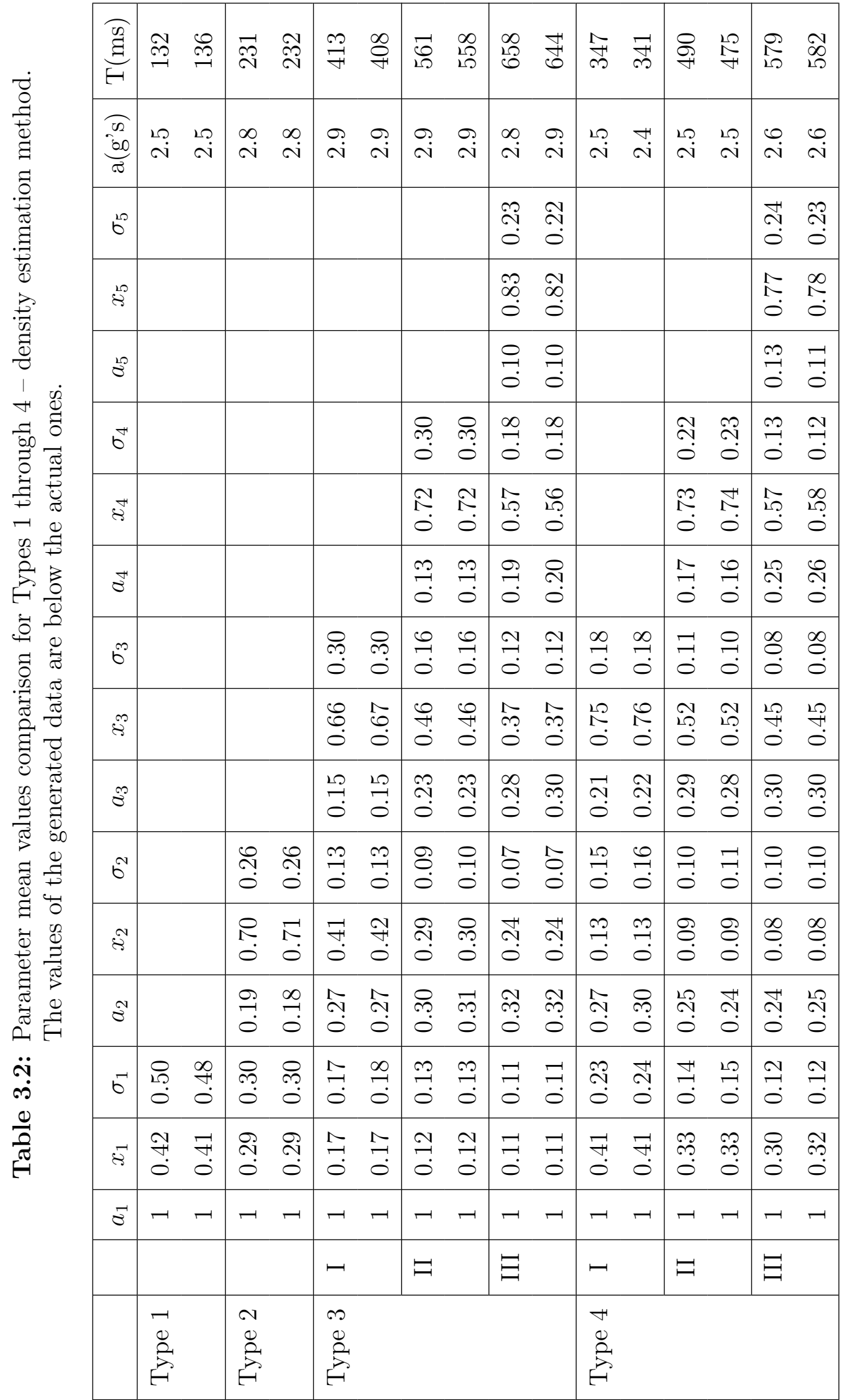




\begin{tabular}{|c|c|c|c|c|c|c|c|c|c|c|c|c|c|c|c|c|c|}
\hline$\underset{H}{\stackrel{a}{g}}$ & 客原 & 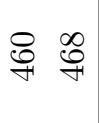 & & $\begin{array}{ll}\text { 触 } & \stackrel{0}{3} \\
\end{array}$ & 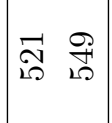 & 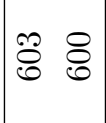 & $\begin{array}{ll}0 & 0 \\
0 & 0\end{array}$ & & & & $\left|\begin{array}{ll}12 & 12 \\
10 & 0 \\
10 & 12\end{array}\right|$ & 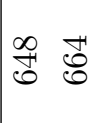 & $\mid$\begin{tabular}{cc}
0 & 0 \\
\multirow{1}{*}{} & 12
\end{tabular} & $\underset{0}{\stackrel{0}{0}} \stackrel{20}{=}$ & $\stackrel{R}{R}$ & 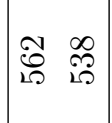 & 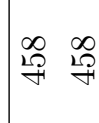 \\
\hline 6 & & & & & & & & & & & 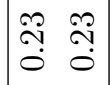 & 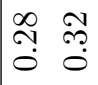 & $\mid$\begin{tabular}{cc}
$\stackrel{\sim}{*}$ & $\stackrel{\sim}{~}$ \\
\hdashline & 0
\end{tabular} & 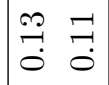 & $\begin{array}{cc}\infty & 1 \\
\stackrel{-1}{0} & \stackrel{0}{0}\end{array}$ & 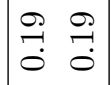 & 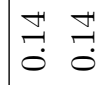 \\
\hline \& & & & & & & & & & & & $\left|\begin{array}{cc}1 & \sim \\
\infty & \infty \\
0 & 0 \\
0 & \dot{0}\end{array}\right|$ & \begin{tabular}{ll}
$\mathcal{N}$ & \multirow{1}{N}{} \\
0 & 0
\end{tabular} & $\mid \begin{array}{ll}\ddot{1} & \mathscr{0} \\
\infty & \infty \\
0 & 0\end{array}$ & $\mid \begin{array}{ll}0 & 0 \\
\infty & 0 \\
0 & \infty \\
0 & 0\end{array}$ & $\mid \begin{array}{ll}\infty & 2 \\
\infty & \infty \\
0 & 0 \\
0 & 0\end{array}$ & $\left|\begin{array}{ll}\mathbb{T} & 0 \\
0 & 0\end{array}\right|$ & $\begin{array}{ll}\infty & \infty \\
\infty & \infty \\
0 & 0 \\
0 & 0\end{array}$ \\
\hline 8 & & & & & & & & & & & $\mid \begin{array}{ll}1 & 1 \\
0 & 0 \\
0 & 0\end{array}$ & શ̂: & 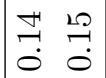 & $\begin{array}{ll}\vec{H} & \overrightarrow{1} \\
\overrightarrow{0} & \overrightarrow{0}\end{array}$ & $\begin{array}{ll}\vec{N} & \stackrel{9}{\sigma} \\
0 & \overrightarrow{0}\end{array}$ & 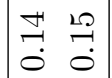 & $\begin{array}{ll}\stackrel{9}{9} & 9 \\
0 & \stackrel{0}{0}\end{array}$ \\
\hline$b^{+H}$ & & & & 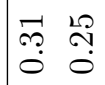 & \begin{tabular}{ll}
\multirow{N}{N}{} & $\vec{n}$ \\
\hdashline & $?$ \\
0
\end{tabular} & \begin{tabular}{ll}
$\infty$ & $\infty$ \\
\multirow{N}{*}{} & $\sim$ \\
0 & $\dddot{0}$
\end{tabular} & $\stackrel{m}{9} \stackrel{m}{0}$ & & & & $\left|\begin{array}{ll}\vec{H} & \vec{I} \\
\ddot{0} & \breve{0}\end{array}\right|$ & $\stackrel{m}{9} \stackrel{12}{0}$ & $\begin{array}{ll}20 & 0 \\
\ddot{0} & \ddots \\
0\end{array}$ & $\begin{array}{cc}0 & \\
\dot{0} & \overrightarrow{0}\end{array}$ & \begin{tabular}{cc}
0 & 1 \\
\hdashline & $\ddots$ \\
0 & 0
\end{tabular} & $\begin{array}{ll}\exists & \exists \\
\ddot{0} & \overrightarrow{0}\end{array}$ & \begin{tabular}{ll}
$\mathscr{C}$ & 8 \\
\hdashline & $\stackrel{0}{0}$
\end{tabular} \\
\hline ¿্ & & & & $\begin{array}{ll}\Re & \sim \\
& 0 \\
0 & 0\end{array}$ & $\begin{array}{ll}R & 0 \\
0 & \stackrel{0}{0} \\
0 & 0\end{array}$ & $\begin{array}{ll}\mathcal{T} & \mathfrak{P} \\
0 & 0\end{array}$ & 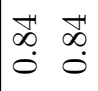 & & & & \begin{tabular}{ll}
$\vec{I}$ & \multicolumn{1}{|}{} \\
0 & 0
\end{tabular} & 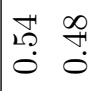 & 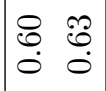 & $\mid \begin{array}{ll}R & \Re \\
0 & 0\end{array}$ & 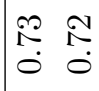 & $\left|\begin{array}{ll}\mathscr{O} & \mathscr{0} \\
\dot{0} & 0 \\
0 & 0\end{array}\right|$ & $\begin{array}{ll}0 & 0 \\
1 & R \\
0 & 0\end{array}$ \\
\hline ठ্ & & & & 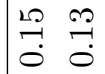 & $\begin{array}{ll}\stackrel{2}{2} & 0 \\
\sim & \sim \\
0 & 0\end{array} \mid$ & 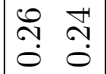 & مि & & & & $\left|\begin{array}{cc}\vec{N} & \vec{N} \\
0 & 0\end{array}\right|$ & 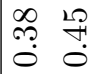 & \begin{tabular}{ll}
2 & $\infty$ \\
$ٌ$ & $?$ \\
\hdashline & 0 \\
0 & 0
\end{tabular} & 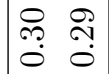 & 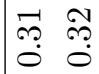 & 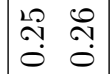 & \begin{tabular}{ll}
$J^{\prime}$ & \multicolumn{1}{c}{} \\
0 & 0 \\
0 & 0
\end{tabular} \\
\hline $0^{\infty}$ & 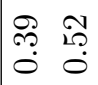 & $\stackrel{\overbrace{}}{\stackrel{2}{0}} \stackrel{9}{0}$ & & $\begin{array}{cc}0 & 1 \\
\stackrel{1}{0} & \breve{0}\end{array}$ & 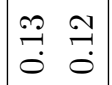 & $\stackrel{I}{\stackrel{I}{*}} \stackrel{I}{0}$ & : & & & & $\left|\begin{array}{ll}\infty & \infty \\
0 & 0 \\
0 & 0 \\
0 & 0\end{array}\right|$ & $\begin{array}{ll}1 & 1 \\
0 & 0 \\
0 & 0\end{array}$ & $\mid \begin{array}{cc}0 & 0 \\
0 & \ddots \\
0 & \breve{0}\end{array}$ & 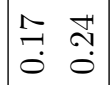 & 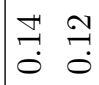 & $\left|\begin{array}{ll}0 & \infty \\
0 & 0 \\
0 & 0\end{array}\right|$ & $\begin{array}{ll}0 & 8 \\
0 & 0 \\
0 & 0\end{array}$ \\
\hline $\mathscr{乛}$ & 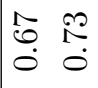 & $\begin{array}{ll}0 & 1 \\
: & 0 \\
0 & 0 \\
0 & 0\end{array}$ & & 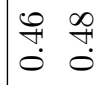 & $\left|\begin{array}{cc}0 & 0 \\
10 & 1 \\
0 & 0 \\
0 & 0\end{array}\right|$ & $\begin{array}{ll}0 & \infty \\
10 & 1 \\
0 & 0 \\
0 & 0\end{array}$ & 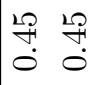 & & & & $\left|\begin{array}{cc}0 & 0 \\
20 & 10 \\
0 & 0 \\
0 & 0\end{array}\right|$ & 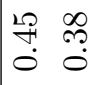 & $\begin{array}{ll}0 & 0 \\
\stackrel{+}{*} & \stackrel{+}{0} \\
0 & 0\end{array}$ & $\left|\begin{array}{cc}20 & 1 \\
10 & 5 \\
0 & 0\end{array}\right|$ & 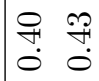 & $\left|\begin{array}{cc}0 & 0 \\
10 & 10 \\
0 & 0\end{array}\right|$ & 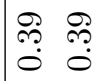 \\
\hline $\mathscr{8}$ & 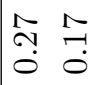 & 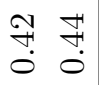 & & 点 & $\begin{array}{cc}\stackrel{1}{\sim} & 0 \\
\stackrel{0}{0} & ٌ \\
0\end{array}$ & 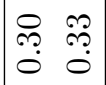 & $\begin{array}{ll}\infty & \infty \\
\stackrel{0}{0} & 0 \\
0 & 0\end{array}$ & & & & $\left|\begin{array}{cc}\vec{N} & \vec{\sim} \\
\ddot{0} & 0\end{array}\right|$ & సै & $\mid \begin{array}{cc}9 & -1 \\
0 & 0 \\
0 & 0\end{array}$ & \begin{tabular}{ll}
$\stackrel{1}{*}$ & $\wp$ \\
\hdashline & 0 \\
0 & 0
\end{tabular} & $\begin{array}{cc}\infty & 0 \\
\stackrel{-1}{0} & \stackrel{1}{0}\end{array}$ & 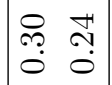 & 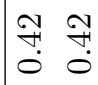 \\
\hline$\tilde{6}$ & $\stackrel{20}{\stackrel{20}{-1}} \stackrel{9}{-1}$ & 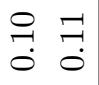 & & 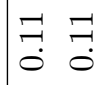 & \begin{tabular}{ll}
$\vec{F}$ & $\overrightarrow{1}$ \\
\hdashline & $\stackrel{0}{0}$
\end{tabular} & $\begin{array}{ll}\infty & \infty \\
0 & 0 \\
0 & 0 \\
0\end{array}$ & $\begin{array}{ll}\infty & \infty \\
0 & 0 \\
0 & 0 \\
0\end{array}$ & & & & $\mid \begin{array}{ll}\vec{H} & \overrightarrow{1} \\
\stackrel{0}{0} & \breve{0}\end{array}$ & $\stackrel{m}{\stackrel{7}{*}} \stackrel{7}{0}$ & $\begin{array}{ll}1 & \hat{0} \\
0 & 0 \\
0 & 0\end{array}$ & $\left|\begin{array}{ll}\infty & \infty \\
0 & 0 \\
0 & 0 \\
0 & 0\end{array}\right|$ & $\begin{array}{ll}\infty & 8 \\
0 & 0 \\
0 & 0 \\
0\end{array}$ & $\left|\begin{array}{cc}0 & 0 \\
\ddots & \ddots \\
0 & \ddots \\
0\end{array}\right|$ & 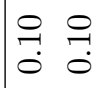 \\
\hline से & 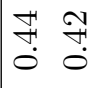 & $\vec{m} \cdot \vec{\leftrightarrow}$ & & 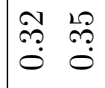 & 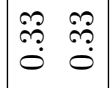 & $\begin{array}{ll}\tilde{N} & \infty \\
\stackrel{N}{0} & \stackrel{0}{0}\end{array}$ & ָ̃ & & & & $\left|\begin{array}{cc}0 & 1 \\
\stackrel{7}{0} & \stackrel{0}{0}\end{array}\right|$ & تै & $\mid$\begin{tabular}{ll}
$\stackrel{H}{N}$ & $\stackrel{\sim}{~}$ \\
\hdashline & 0
\end{tabular} & 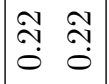 & 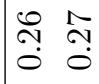 & $\left|\begin{array}{ll}9 & 8 \\
0 & 0 \\
0 & 0\end{array}\right|$ & $\begin{array}{cc}\stackrel{0}{1} & \stackrel{7}{1} \\
0 & \stackrel{0}{0}\end{array}$ \\
\hline $\mathcal{S}$ & $\begin{array}{cc}0 & 0 \\
0.0 & 0 \\
0 & 0 \\
0 & 0\end{array}$ & \begin{tabular}{ll}
12 & 0 \\
\hdashline & $?$ \\
0 & 0 \\
0
\end{tabular} & & \begin{tabular}{ll}
$\stackrel{1}{10}$ & \multicolumn{7}{|c}{} \\
0 & 0
\end{tabular} & \begin{tabular}{ll}
\multirow{N}{N}{} & \multirow{N}{*}{} \\
0 & 0
\end{tabular} & $\begin{array}{ll}\Re & \Re \\
\stackrel{2}{0} & \stackrel{0}{0}\end{array}$ & 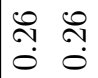 & & & & $\mid \begin{array}{cc}\infty & \infty \\
20 & 0 \\
0 & 0 \\
0 & 0\end{array}$ & 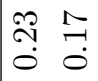 & $\begin{array}{ll}\stackrel{1}{N} & 10 \\
\stackrel{0}{0} & \breve{0}\end{array}$ & 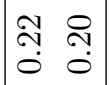 & 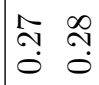 & $\left|\begin{array}{ll}\infty & \infty \\
0 & 0 \\
0 & 0 \\
0 & 0\end{array}\right|$ & $\vec{\sigma} \cdot \vec{\sigma}$ \\
\hline 5 & $\stackrel{m}{\because}:$ & 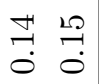 & & $\exists \stackrel{m}{\rightrightarrows}$ & 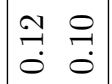 & $\vec{F} \cdot \vec{F}$ & $\begin{array}{ll}9 & 9 \\
0 & 0 \\
0 & 0\end{array}$ & & & & $\begin{array}{ll}ت & F \\
\ddot{0} & \overrightarrow{0}\end{array}$ & $\stackrel{m}{9} \stackrel{2}{\hdashline}$ & $\mid \begin{array}{cc}0 & 0 \\
\vdots & 0 \\
0 & \ddots \\
0\end{array}$ & $\left|\begin{array}{cc}0 & 0 \\
\hdashline & 0 \\
0 & \vdots \\
0 & 0\end{array}\right|$ & $\begin{array}{ll}\Rightarrow & 0 \\
0 & \ddots \\
0\end{array}$ & $\begin{array}{ll}ت & F \\
\vdots & \overrightarrow{0}\end{array}$ & $\begin{array}{cc}\exists & 7 \\
0 & \vdots \\
0\end{array}$ \\
\hline $\overrightarrow{8}$ & 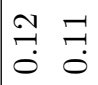 & $\stackrel{m}{\dddot{0}} \stackrel{m}{0}$ & & $\exists \stackrel{I}{\exists}$ & $\begin{array}{ll}\exists & 0 \\
\ddot{0} & \ddots \\
0\end{array}$ & $\exists=$ & $\begin{array}{ll}8 & 9 \\
0 & 8 \\
0\end{array}$ & & & & $\left|\begin{array}{cc}0 & 0 \\
\vdots & \ddots \\
0 & \vdots \\
0\end{array}\right|$ & $\begin{array}{ll}\exists & 7 \\
0 & 0 \\
0\end{array}$ & $\mid \begin{array}{ll}0 & 8 \\
0 & 0 \\
0 & 0\end{array}$ & $\begin{array}{ll}\stackrel{1}{*} & 0 \\
\ddot{0} & \ddots \\
0\end{array}$ & 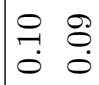 & $\left|\begin{array}{ll}2 & 0 \\
ٌ & 0 \\
0 & 0\end{array}\right|$ & \begin{tabular}{ll}
$\bullet$ & $\bullet$ \\
\hdashline & $\ddots$ \\
0 & $\stackrel{0}{0}$
\end{tabular} \\
\hline $\bar{\sigma}$ & $-r$ & $r-$ & & $-r$ & $-r-1$ & $-r$ & $-r$ & & & & 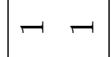 & $-r$ & $-r-1$ & $-1-1$ & $-r-1$ & 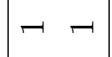 & $-r-1$ \\
\hline 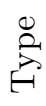 & $\stackrel{I}{I}$ & $\vec{~}$ & $\stackrel{亡}{\grave{1}}$ & $\vec{p}$ & $\stackrel{-}{\stackrel{-}{+}}$ & $\begin{array}{l}\sim \\
\text { N }\end{array}$ & $\frac{i}{\infty}$ & $\stackrel{\vec{I}}{\stackrel{\longrightarrow}{+}}$ & Pi & $\begin{array}{l}\hat{i} \\
\text { N }\end{array}$ & $\underset{:}{=}$ & 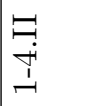 & $\vec{a}$ & 岁 & $\underset{⿱ 亠 䒑}{\stackrel{\sim}{\dot{m}}}$ & 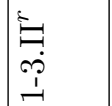 & 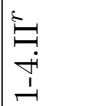 \\
\hline
\end{tabular}




\begin{tabular}{|c|c|c|c|c|c|c|c|c|c|c|c|c|c|c|c|c|c|}
\hline 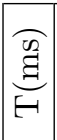 & 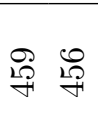 & 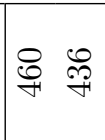 & & 突 & 거소 & $\begin{array}{ll}0 & 0 \\
0 & 0\end{array} \mid$ & $\ddot{8}:$ & & & & $\left|\begin{array}{ll}10 & 120 \\
20 & 00 \\
10 & 10\end{array}\right|$ & $\begin{array}{ll}\stackrel{\infty}{0} & \overrightarrow{8}\end{array}$ & $\left|\begin{array}{ll}0 & 8 \\
0 & 8 \\
0 & 0\end{array}\right|$ & 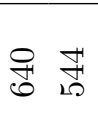 & $\begin{array}{ll}R & 8 \\
0 & 1\end{array}$ & 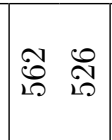 & $\mid$\begin{tabular}{ll}
$\infty$ & $\infty$ \\
12 & 1 \\
\hdashline & 18 \\
+
\end{tabular} \\
\hline 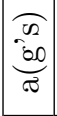 & 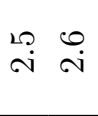 & $\begin{array}{ll}\vec{\sim} & \vec{\Delta} \\
\stackrel{\sim}{*}\end{array}$ & & 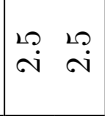 & 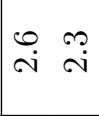 & $\begin{array}{cc}\infty & \infty \\
i & \stackrel{i}{v}\end{array} \mid$ & 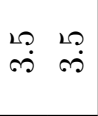 & & & & $\left|\begin{array}{cc}0 & 0 \\
\dot{S O} & \dot{\infty}\end{array}\right|$ & 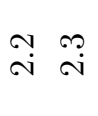 & $\left|\begin{array}{rr}\stackrel{H}{*} & \vec{v}\end{array}\right|$ & 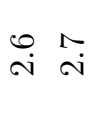 & 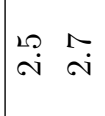 & $\left|\begin{array}{ll}\vec{H} & \vec{v} \\
\stackrel{\sim}{*} & .\end{array}\right|$ & 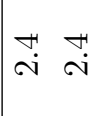 \\
\hline 6 & & & & & & & & & & & $\begin{array}{ll}\Re & \Re \\
\stackrel{N}{N} & \stackrel{0}{0}\end{array}$ & 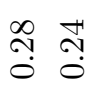 & 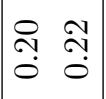 & $\stackrel{m}{\dddot{2}} \stackrel{-}{0}$ & $\stackrel{\infty}{\stackrel{\infty}{*}} \stackrel{2}{\stackrel{9}{0}}$ & 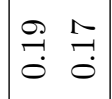 & 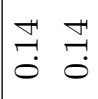 \\
\hline 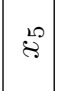 & & & & & & & & & & & $\left|\begin{array}{cc}\infty & 1 \\
\infty & \infty \\
0 & 0\end{array}\right|$ & 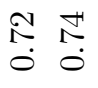 & $\left|\begin{array}{ll}0 & \overrightarrow{1} \\
0 & \infty \\
0 & 0\end{array}\right|$ & $\begin{array}{ll}\infty & \hat{\infty} \\
\infty & \infty \\
0 & 0 \\
0 & 0\end{array}$ & $\begin{array}{ll}0 & 10 \\
\infty & \infty \\
0 & 0\end{array}$ & $\left|\begin{array}{cc}\stackrel{0}{I} & \overrightarrow{0} \\
0 & 0\end{array}\right|$ & $\mid \begin{array}{ll}\infty & \infty \\
\infty & \infty \\
0 & 0 \\
0 & 0\end{array}$ \\
\hline 8 & & & & & & & & & & & $\left|\begin{array}{ll}1 & 1 \\
0 & 0 \\
0 & 0\end{array}\right|$ & 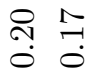 & 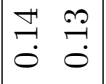 & 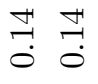 & $\begin{array}{ll}\vec{N} & \varrho \\
\stackrel{-}{0} & \stackrel{0}{0}\end{array}$ & 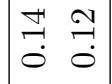 & 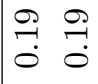 \\
\hline$b^{+}$ & & & & 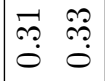 & 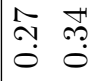 & 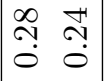 & 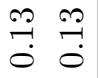 & & & & $\mid$\begin{tabular}{cc}
$\vec{H}$ & $\vec{Z}$ \\
\hdashline & $\vdots$ \\
0 & 0
\end{tabular} & 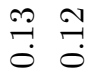 & 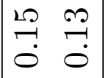 & 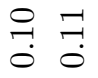 & 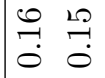 & $\left|\begin{array}{cc}\exists & \exists \\
0 & \exists \\
0\end{array}\right|$ & $\begin{array}{ll}\mathscr{b} & 8 \\
0 & 0\end{array}$ \\
\hline ङ્ & & & & 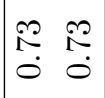 & $\begin{array}{ll}R & 0 \\
1 & 0 \\
0 & 0\end{array}$ & $\begin{array}{ll}N & F \\
0 & 0\end{array}$ & $\begin{array}{ll}+\infty & + \\
\infty & \infty \\
0 & 0\end{array}$ & & & & \begin{tabular}{ll}
$\check{I}$ & \multicolumn{1}{|c}{} \\
0 & 0
\end{tabular} & $\begin{array}{ll}\overrightarrow{1} & 0 \\
\stackrel{0}{0} & 0 \\
0 & 0\end{array}$ & $\begin{array}{ll}0 & \ddots \\
0 & 0 \\
0 & 0\end{array}$ & $\begin{array}{ll}R & 0 \\
0 & 0 \\
0 & 0\end{array}$ & 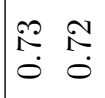 & $\left|\begin{array}{ll}3 & 0 \\
0 & 0 \\
0 & 0\end{array}\right|$ & $\begin{array}{ll}0 & 0 \\
0 & 0 \\
0 & 0\end{array}$ \\
\hline है & & & & 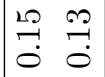 & 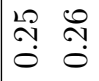 & $\begin{array}{lll}0 & \stackrel{2}{2} \\
\stackrel{2}{*} & \stackrel{0}{0}\end{array}$ & 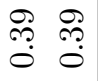 & & & & $\left|\begin{array}{ll}\vec{N} & \vec{\jmath} \\
0 & 0\end{array}\right|$ & 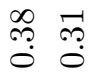 & 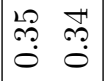 & 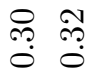 & \begin{tabular}{ll}
$\overrightarrow{3}$ & \multirow{2}{*}{} \\
0 & 0
\end{tabular} & \begin{tabular}{ll}
$\stackrel{2}{P}$ & $\vec{\jmath}$ \\
\hdashline & 0
\end{tabular} & 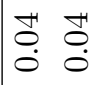 \\
\hline $0^{\circ}$ & 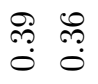 & 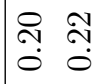 & & $\begin{array}{cc}0 & 0 \\
\stackrel{1}{0} & \stackrel{1}{0}\end{array}$ & 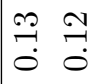 & 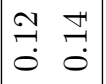 & $\begin{array}{ll}\Re & \Re \\
\stackrel{2}{*} & \stackrel{0}{0}\end{array}$ & & & & $\left|\begin{array}{ll}\infty & \infty \\
0 & 0 \\
0 & 0 \\
0 & 0\end{array}\right|$ & $\begin{array}{ll}1 & \infty \\
0 & 0 \\
0 & 0 \\
0\end{array}$ & $\left|\begin{array}{ll}g & 0 \\
0 & \ddots \\
0 & 0\end{array}\right|$ & $\stackrel{ㄷ}{\stackrel{2}{0}} \stackrel{9}{0}$ & $\begin{array}{ll}\vec{H} & \overrightarrow{1} \\
\stackrel{0}{0} & \stackrel{0}{0}\end{array}$ & $\left|\begin{array}{ll}0 & 0 \\
0 & 0 \\
0 & 0\end{array}\right|$ & $\mid \begin{array}{ll}\mathscr{8} & 8 \\
0 & 0 \\
0 & 0\end{array}$ \\
\hline : & 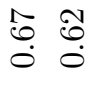 & $\begin{array}{ll}0 & 0 \\
0 & 0 \\
0 & 0\end{array}$ & & 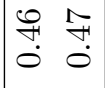 & 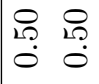 & $\left|\begin{array}{cc}0 & 9 \\
0 & 0 \\
0 & 0 \\
0 & 0\end{array}\right|$ & 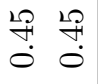 & & & & $\left|\begin{array}{cc}0 & 0 \\
10 & 1 ? \\
0 & 0\end{array}\right|$ & 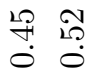 & \begin{tabular}{cc}
0 & \multicolumn{1}{|c}{} \\
$\stackrel{+}{0}$ & $\stackrel{0}{0}$
\end{tabular} \mid & 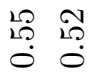 & $\mid \begin{array}{ll}\stackrel{f}{4} & ? \\
0 & \stackrel{1}{0}\end{array}$ & 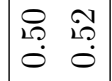 & مै \\
\hline$\Re$ & 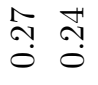 & 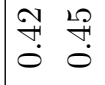 & & 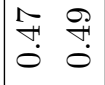 & 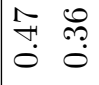 & 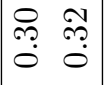 & $\begin{array}{ll}\infty & \infty \\
0 & \infty \\
0 & 0 \\
0\end{array}$ & & & & $\left|\begin{array}{cc}\vec{N} & \vec{N} \\
0 & 0\end{array}\right|$ & $\begin{array}{ll}\stackrel{2}{N} & \Re \\
\stackrel{0}{0} & 0\end{array}$ & 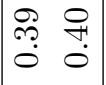 & \begin{tabular}{ll}
$\stackrel{\Re}{N}$ & $\stackrel{9}{0}$ \\
\hdashline & 0
\end{tabular} & 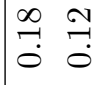 & 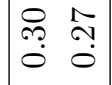 & 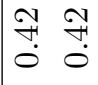 \\
\hline$\delta$ & \begin{tabular}{ll}
10 & 9 \\
\hdashline & 9 \\
0 & 0
\end{tabular} & $\begin{array}{cc}0 & 0 \\
\ddots & \ddots \\
0 & 0\end{array}$ & & 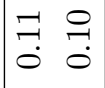 & 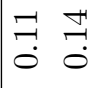 & $\left|\begin{array}{ll}\infty & 9 \\
0 & 0 \\
0 & 0\end{array}\right|$ & $\begin{array}{ll}\infty & \infty \\
0 & 0 \\
0 & 0 \\
0\end{array}$ & & & & $\left|\begin{array}{cc}\vec{H} & \overrightarrow{7} \\
\hdashline & 0\end{array}\right|$ & $\stackrel{m}{\rightarrow} \stackrel{20}{\longrightarrow} \underset{0}{0}$ & $\left|\begin{array}{ll}1 & 0 \\
0 & 0 \\
0 & 0\end{array}\right|$ & $\begin{array}{ll}\infty & \infty \\
0 & \infty \\
0 & 0 \\
0\end{array}$ & $\mid \begin{array}{ll}\infty & \infty \\
0 & 0 \\
0 & 0 \\
0\end{array}$ & $\left|\begin{array}{cc}0 & 0 \\
\hdashline & \ddots \\
0 & 0 \\
0\end{array}\right|$ & \begin{tabular}{cc}
0 & 0 \\
\hdashline & $\ddots$ \\
0 & 0
\end{tabular} \\
\hline \&્ه & 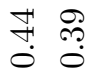 & $\begin{array}{ll}\ddot{m} & \vec{n} \\
\ddot{0} & \ddot{\sigma}\end{array}$ & & 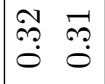 & :ִ & $\begin{array}{cc}\hat{N} & \hat{N} \\
0 & 0\end{array}$ & 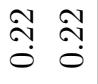 & & & & 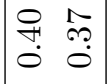 & 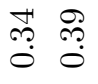 & $\left|\begin{array}{ll}\stackrel{H}{N} & \stackrel{N}{N} \\
\hdashline & 0\end{array}\right|$ & 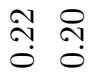 & \begin{tabular}{ll}
$\stackrel{1}{N}$ & $\stackrel{2}{~}$ \\
\hdashline & 0
\end{tabular} & $\left|\begin{array}{ll}g & g \\
0 & 0 \\
\dot{0} & \dot{0}\end{array}\right|$ & $\begin{array}{ll}0 & 0 \\
\ddots & \ddots \\
0 & 0 \\
0\end{array}$ \\
\hline $\mathcal{S}$ & 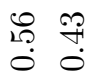 & 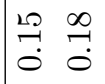 & & $\begin{array}{cc}\vec{H} & 0 \\
0 & 0 \\
0 & 0\end{array}$ & Î & 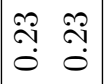 & $\begin{array}{ll}\stackrel{2}{N} & \stackrel{2}{1} \\
0 & 0\end{array}$ & & & & $\left|\begin{array}{cc|}\infty & \infty \\
19 & 10 \\
0 & 0 \\
0 & 0\end{array}\right|$ & 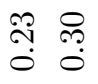 & $\left|\begin{array}{cc}0 & \infty \\
\sim & \cdots \\
0 & 0\end{array}\right|$ & \begin{tabular}{ll}
\multirow{N}{*}{} & \multirow{2}{*}{} \\
\hdashline & 0
\end{tabular} & \begin{tabular}{ll}
$N$ & 0 \\
\multirow{N}{*}{} & $\stackrel{0}{0}$
\end{tabular} & $\left|\begin{array}{ll}\infty & 0 \\
0 & 1 \\
0 & 0\end{array}\right|$ & $\begin{array}{cc}\sigma & \sigma \\
\dot{\sigma} & \vdots \\
0\end{array}$ \\
\hline$\vec{b}$ & 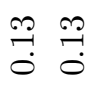 & 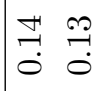 & & $\exists=$ & $\stackrel{ }{\stackrel{\circ}{*}} \stackrel{?}{\circ}$ & 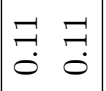 & $\begin{array}{ll}2 & 9 \\
0 & 0 \\
0 & 0\end{array}$ & & & & $\begin{array}{ll}\exists & \ddots \\
0 & \ddots \\
0 & 0\end{array}$ & 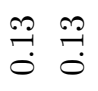 & $\left|\begin{array}{ll}0 & 0 \\
\hdashline & 0 \\
0 & 0\end{array}\right|$ & 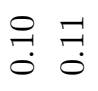 & $\exists=$ & \begin{tabular}{cc}
$\exists$ & - \\
\hdashline & $\ddots$ \\
0 & 0
\end{tabular} & 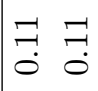 \\
\hline $\overrightarrow{8}$ & 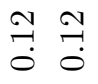 & $\stackrel{m}{\stackrel{2}{\rightleftarrows}} \stackrel{\overbrace{}}{0}$ & & $\exists=$ & $\begin{array}{cc}\Rightarrow & 0 \\
\stackrel{0}{0} & 0 \\
0\end{array}$ & $\begin{array}{ll}\exists & \exists \\
\vdots & \ddots \\
0 & 0\end{array}$ & $\begin{array}{ll}8 & 9 \\
0 & 0 \\
0 & 0\end{array}$ & & & & $\left|\begin{array}{cc}0 & 0 \\
\hdashline & \ddots \\
0 & 0 \\
0\end{array}\right|$ & 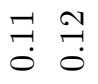 & $\left|\begin{array}{ll}g & 0 \\
0 & 0 \\
0 & 0\end{array}\right|$ & 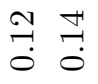 & $\mid \begin{array}{cc}0 & 0 \\
\ddots & \ddots \\
0 & \ddots \\
0\end{array}$ & 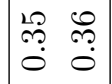 & 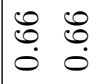 \\
\hline$\vec{\sigma}$ & $\neg-r$ & $\neg-$ & & $-r-$ & $-r-1$ & $\begin{array}{lll}-1 & -1\end{array}$ & $-r$ & & & & $\begin{array}{ll}-1 & -1\end{array}$ & $\neg-$ & $-\neg$ & $-r$ & $\neg-$ & $\neg-1$ & $\neg-1$ \\
\hline 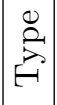 & $\stackrel{1}{i}$ & ล่ & ì & 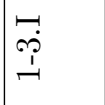 & $\stackrel{\longrightarrow}{\stackrel{4}{+}}$ & à & $\underset{m}{\vec{I}}$ & $\stackrel{I}{\stackrel{I}{+}}$ & Pr & $\begin{array}{l}\grave{1} \\
\text { जे } \\
\text { in }\end{array}$ & 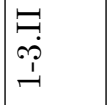 & 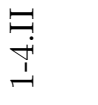 & 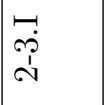 & ঙr & 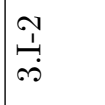 & 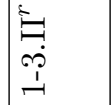 & 点 \\
\hline
\end{tabular}


Table 3.5: Prescribed vs detected profiles.

\begin{tabular}{|c|c|c|c|}
\hline Type & Prescribed & & Detected \\
\hline & & Copula Method & Density Estimation Method \\
\hline 1 & 22 & 22 & 22 \\
\hline 2 & 227 & 212 & 213 \\
\hline 3.I & 566 & 561 & 547 \\
\hline 3.II & 644 & 606 & 641 \\
\hline 3.III & 84 & 85 & 80 \\
\hline 4.I & 29 & 25 & 27 \\
\hline 4.II & 68 & 47 & 58 \\
\hline 4.III & 58 & 58 & 63 \\
\hline $1-2$ & 4 & 4 & 5 \\
\hline $2-1$ & 5 & 6 & 5 \\
\hline $1-2^{r}$ & 0 & 3 & 2 \\
\hline 1-3.I & 10 & 12 & 13 \\
\hline 1-4.I & 0 & 0 & 0 \\
\hline $2-2$ & 31 & 29 & 30 \\
\hline 3.I-1 & 0 & 0 & 0 \\
\hline 4.I-1 & 0 & 0 & 0 \\
\hline $1-3 . I^{r}$ & 0 & 2 & 0 \\
\hline $2-2^{r}$ & 0 & 1 & 0 \\
\hline 1-3.II & 2 & 2 & 4 \\
\hline 1-4.II & 3 & 3 & 3 \\
\hline 2-3.I & 8 & 12 & 9 \\
\hline 2-4.I & 6 & 6 & 8 \\
\hline $3 . \mathrm{I}-2$ & 10 & 9 & 5 \\
\hline $1-3 . \mathrm{II}^{r}$ & 5 & 3 & 5 \\
\hline 1-4.II ${ }^{r}$ & 2 & 2 & 2 \\
\hline
\end{tabular}




\subsection{Discussion}

The different aspects of the synthetic pulses are discussed in the first part of this section. The inter-pulse characteristics are discussed in the second part.

\subsubsection{On Synthetic Pulses}

The produced synthetic pulses have the same dependence structure as shown in Figures 3.5 and 3.6. This shows that both the method using copulas and the density estimation method were appropriate. The advantage of using copulas is the fact that this method has the potential to be fully parametric. The dependence between parameters is contained in the Gaussian copula, which is parameterized by the correlation matrix of the data. If an appropriate model can be found for the individual parameter distributions, then the method using copulas would be fully parametric.

The individual parameter distributions have been captured as well by both methods, as shown in Figures 3.8 and 3.9. The parameter mean values of the simulated data are in good agreement with the mean values of the actual parameters, as shown in Tables 3.1 through 3.4.

The synthetic pulses were fed back to the curve fitting algorithm and a detection rate of $95 \%$ was found. In curve fitting the synthetic pulses, a lower threshold was set

for the residual defining an adequate fit (average residual threshold of 0.001). This is because the synthetic pulses are expected to match closely the fitted curves. If a less strict threshold is used, the detection rate increases. However, a misinterpretation of the different types could occur (e.g., Type 3.I detected as Type 2 due to a high threshold). In Table 3.5, it can be seen that the number of prescribed pulses of each 
type matches well the number of detected pulses of each type.

\subsubsection{On Inter-Pulse Characteristics}

The produced acceleration time history exhibits no significant drift in either velocity nor displacement as shown in Figure 3.4. The displacement is within the range of \pm 1 metre for the most part, which is consistent with a wave height of 2 metres. Downward accelerations are lower than $-1 \mathrm{~g}$ in a small number of cases. This occurs when a reasonable solution (i.e. large variation in displacement or significant drift) cannot be obtained with the cam profile $y_{\text {cam }}$, which was described in Section 3.2. The boat experiences mainly the pull of gravity. However, a larger downwards force is possible as a result of combined rotational and translational motion. This occurs when the forward portion of the boat pitches and heaves about a rotation point close to the centre of the boat, right before impact.

\subsection{Conclusion}

Data simulation procedures were employed to generate HSC acceleration time histories. It was shown that the characteristics of the simulated data are reasonably close to those of the actual data. Simulated time histories are useful for extending sea trial time histories by using their statistical characteristics and to determine the risk of injury of human occupants by computing measures such as vibration dose. 


\section{Chapter 4}

\section{Conclusion}

The analysis of RHIB acceleration data yielded a five-type classification of impact acceleration pulses. It was found that the different profiles can be characterized by a combination of modified Gaussian curves. Equation parameters were obtained from curve fitting the data. The mean values of these parameters were provided and can be used to obtain standard profiles for testing RHIB seats. The developed profile detection algorithm is also intended as a general tool for the analysis of HSC acceleration

data. In addition to pulse characterization, two methods for synthesizing acceleration time histories with similar characteristics to the original data were presented. Both methods were tested and shown to be reliable. Synthetic time histories can be used for numerical simulation of HSC seat response. Vibration and shock measures can then be computed such as vibration dose when sea trial data is not available or when it is required to extend short time histories. The main contributions of this work are:

1. Mathematical characterization of HSC acceleration pulses; 
2. Classification of acceleration pulses into different profiles;

3. Development of an algorithm for the analysis of acceleration data; and

4. Development of an algorithm for the synthesis of acceleration time histories with similar pulse shape characteristics to actual data.

To the best of the author's knowledge, the contributions of this work are novel.

Using the work presented in this thesis, several follow-on projects can be conducted in the spirit of HSC shock mitigation:

1. An assessment of the severity of the different profiles can be made by subjecting HSC seats to these profiles. A test rig is, in fact, currently being built at Carleton University for this purpose.

2. Relationships between pulse shape characteristics and boat/sea parameters can be investigated. This can be achieved by applying the developed curve fitting algorithm to acceleration data of different types of HSC and in different sea conditions. Establishing such correlations could yield a useful tool for boat and seat design. 


\section{References}

[1] Odd M. Faltinsen. Hydrodynamics of High-Speed Marine Vehicles. Cambridge University Press, 2006.

[2] Jonathan M. Ross. Human Factors for Naval Marine Vehicle Design and Operation. Ashgate, 2009.

[3] Sean D. Kearns. Analysis and mitigation of mechanical shock effects on high speed planing boat. Master's thesis, MIT, 2001.

[4] Ian Rowley Trevor Dobbins and Lorne Campbell. High Speed Craft Human Factors Engineering Design Guide ABCD Group. Human Sciences and Engineering Ltd, 2008.

[5] Damen shipyard group website http://www.damen.com/en/markets/defenceand-security.

[6] Titan boats website http://www.titanboats.com.

[7] The Specialist Committee on High Speed Craft. Final report and recommendations. In 26th ITTC International Towing Tank Conference, 2011.

[8] P. A. Wilson N. C. Townsend and S. Austen. What influences rigid inflatable boat motions? Engineering for the Maritime Environment, 222:207-217, 2008.

[9] Edward M. Lewandowski. The Dynamics of Marine Craft. World Scientific, 2004.

[10] J.R. Shahraki at al. Prediction of slamming behaviour of monohull and multihull forms using smoothed particle hydrodynamics. In IX HSMV, 2011. 
[11] Stephen Myers Trevor Dobbins and Rosemary Dyson. High speed craft motion analysis impact count index (ici). In United Kingdom Conference on Human Responses to Vibration, 2008.

[12] S. J. C. Dyne J. M. Hirst and T. E. Coe. High speed craft motion analysis impact count index (ici). In SURV 8-Surveillance, Search and Rescue Craft, 2013.

[13] Jacques Georgy Aboelmagd Noureldin, Tashfeen B. Karamat. Fundamentals of INS, GPS and Their Integration. Springer, 2012.

[14] Roger B. Nelsen. An Introduction to Copulas. Springer, 2006.

[15] http://www.mathworks.com/help/stats/examples/simulating-dependentrandom-variables-using-copulas.html.

[16] Johann C. Strelen. Tools for dependent simulation input with copulas. In Simulation Tools and Techniques, 2009.

[17] Bernard W. Silverman. Density Estimation for Statistics and Data Analysis. Chapman and Hall, 1986.

[18] Wendy L . Martinez and Angel R . Martinez. Computational Statistics Handbook with MATLAB. Chapman and Hall, 2001. 


\section{Appendix A}

\section{Reduction of Additional Data Set}

An additional data set was provided to the ADL by DRDC. The sensors used here were:

- 10 Silicon Designs 2460 triaxial accelerometers, using a sampling rate of $4000 \mathrm{~Hz}$; and

- 1 Microstrain 3DM-GX3-25 Attitude Heading Reference System (RS232/USB \#6223-4220) Gyroscope of unknown sampling rate. The accelerometer data was down sampled to $100 \mathrm{~Hz}$ and the gyroscope data was interpolated in order to be aligned with the accelerometer data. Sea trials were performed over a number of days. The results shown below are for the sea trial performed on October $24^{\text {th }}, 2012$ and for the acceleration at the forward deck of the boat.

The profile distribution seen in this data is not identical to the data set presented in the thesis. The main differences are: a smaller number of Type 3.II and 3.III profiles, a larger number of Type 1 and 4.I profiles. While the shape parameters of the pulses seen in both data sets are within the same range, significant differences can be observed between the peak accelerations and pulse durations of each set. 


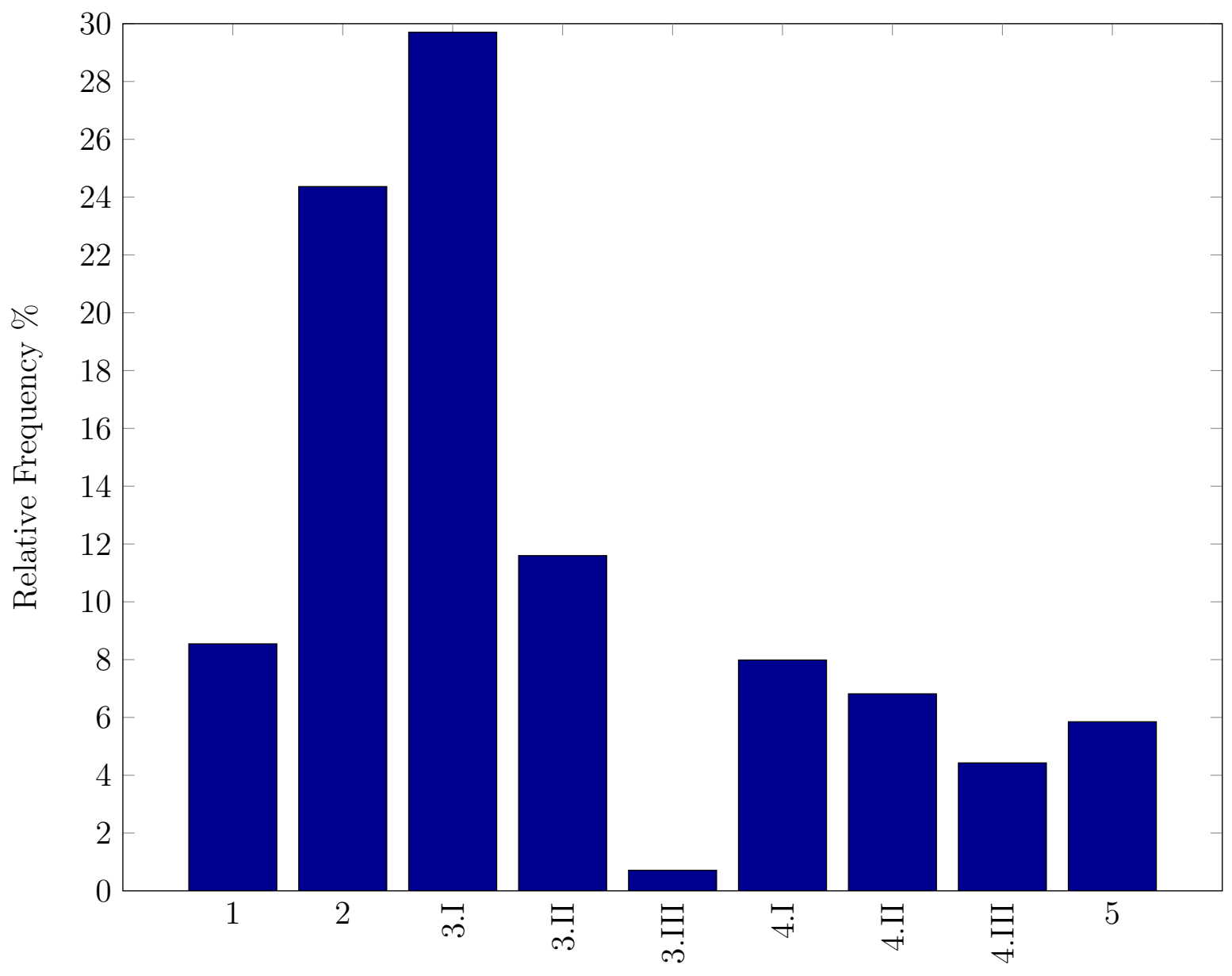

Figure A.1: Profile distribution - forward position. 


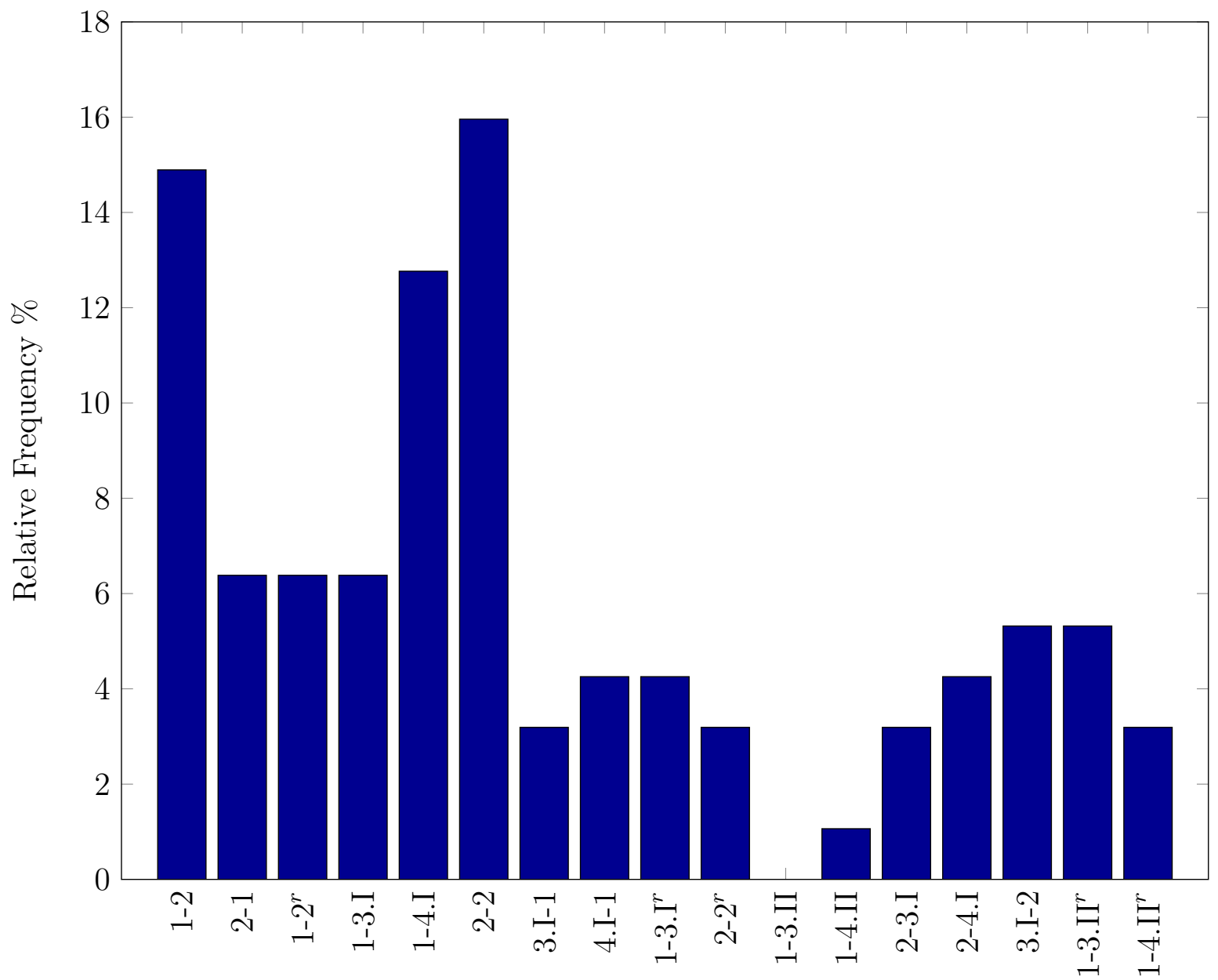

Figure A.2: Distribution of Type 5 profiles - forward position. 


\begin{tabular}{|c|c|c|c|c|c|c|c|c|}
\hline $\begin{array}{l}\stackrel{\sigma \Omega}{\Xi} \\
E\end{array}$ & $\stackrel{0}{=}$ & 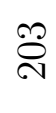 & $\begin{array}{l}\infty \\
\infty \\
\sim \\
N\end{array}$ & कै & $\stackrel{\text { ㄱ }}{\sim}$ & 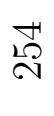 & $\begin{array}{l}\infty \\
\text { NN} \\
\text { Non }\end{array}$ & $\begin{array}{l}\infty \\
\stackrel{\infty}{\infty} \\
\infty\end{array}$ \\
\hline$\frac{\overparen{\pi}}{\overbrace{\pi 0}^{\infty 00}}$ & $\underset{\infty}{0}$ & $\stackrel{N}{\infty}$ & $\stackrel{\sim}{\infty}$ & $\underset{\infty}{\circ}$ & $\underset{\leftrightarrow}{\circ}$ & $\stackrel{\theta}{\sim}$ & $\begin{array}{l}\infty \\
\sim \\
\sim\end{array}$ & $\vec{r}$ \\
\hline $6^{10}$ & & & & & $\stackrel{\vec{H}}{\stackrel{4}{0}}$ & & & $\stackrel{N}{\circ}$ \\
\hline$\stackrel{10}{20}$ & & & & & 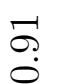 & & & $\begin{array}{l}0 \\
\infty \\
0\end{array}$ \\
\hline 8 & & & & & $\stackrel{\infty}{\stackrel{\infty}{0}}$ & & & $\stackrel{\infty}{\ddot{0}}$ \\
\hline$b^{+t}$ & & & & 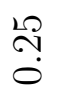 & $\stackrel{\infty}{\stackrel{\infty}{0}}$ & & $\stackrel{\Re}{\stackrel{?}{0}}$ & 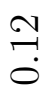 \\
\hline हु & & & & $\begin{array}{l}\stackrel{\text { ov }}{\infty} \\
\dot{0}\end{array}$ & $\stackrel{R}{R}$ & & $\begin{array}{l}\infty \\
\infty \\
0\end{array}$ & $\begin{array}{l}\tilde{0} \\
0\end{array}$ \\
\hline है & & & & $\underset{\dot{0}}{\stackrel{H}{\prime}}$ & $\stackrel{\overbrace{}}{\mathfrak{O}}$ & & $\stackrel{\infty}{\stackrel{\infty}{0}}$ & 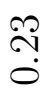 \\
\hline$b^{\infty}$ & & & $\begin{array}{l}\infty \\
\stackrel{\sim}{0}\end{array}$ & $\stackrel{10}{\ddot{0}}$ & $\stackrel{\sim}{\stackrel{0}{0}}$ & 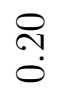 & $\stackrel{\stackrel{\sim}{Z}}{\circ}$ & $\begin{array}{l}\mathscr{Q} \\
\dot{0}\end{array}$ \\
\hline ॄై & & & $\stackrel{10}{\stackrel{10}{0}}$ & $\begin{array}{l}0 \\
\stackrel{0}{0} \\
0\end{array}$ & 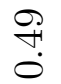 & $\stackrel{0}{L}$ & î & i⿱⺈ \\
\hline రొ & & & $\stackrel{\infty}{\stackrel{\infty}{0}}$ & $\ddot{\oplus}$ & $\stackrel{\text { ๙ै }}{0}$ & $\stackrel{\overbrace{}}{\stackrel{0}{0}}$ & $\stackrel{10}{\stackrel{2}{0}}$ & $\stackrel{?}{?}$ \\
\hline$\sigma^{N}$ & & $\stackrel{N}{\stackrel{N}{0}}$ & $\stackrel{10}{\stackrel{10}{0}}$ & $\ddot{G}$ & $\begin{array}{l}\mathscr{O} \\
\stackrel{0}{0}\end{array}$ & $\stackrel{H}{\stackrel{H}{0}}$ & $\stackrel{\stackrel{\sim}{Z}}{\stackrel{0}{0}}$ & $\stackrel{m}{\stackrel{2}{0}}$ \\
\hline से & & $\begin{array}{l}\infty \\
0 \\
0\end{array}$ & 뭉 & 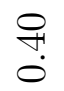 & $\ddot{\leftrightarrow}$ & $\stackrel{\vec{H}}{\stackrel{0}{0}}$ & $\ddot{\rightleftarrows}$ & $\stackrel{\circ}{\stackrel{1}{0}}$ \\
\hline $\mathcal{O}$ & & 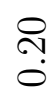 & $\begin{array}{l}\stackrel{0}{~} \\
\stackrel{0}{0}\end{array}$ & $\stackrel{\stackrel{H}{N}}{0}$ & \begin{tabular}{l}
$\infty$ \\
$\stackrel{\sim}{0}$ \\
\hdashline
\end{tabular} & $\stackrel{0}{\overparen{0}}$ & $\stackrel{\circ}{\overrightarrow{0}}$ & ન્ \\
\hline$\sqrt{6}$ & $\begin{array}{l}0 \\
1 ? \\
0\end{array}$ & $\begin{array}{l}\infty \\
\stackrel{N}{0} \\
0\end{array}$ & $\stackrel{\Omega}{\stackrel{0}{0}}$ & $\stackrel{ }{\stackrel{1}{0}}$ & $\stackrel{\vec{t}}{\stackrel{0}{0}}$ & 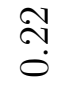 & $\stackrel{\infty}{\rightarrow}$ & $\stackrel{10}{\stackrel{10}{0}}$ \\
\hline$\vec{~}$ & $\begin{array}{l}0 \\
\stackrel{1}{1} \\
0\end{array}$ & $\stackrel{\leftrightarrow}{0}$ & $\stackrel{\text { Iิ }}{\stackrel{0}{0}}$ & $\stackrel{\overbrace{}}{\stackrel{\overbrace{}}{0}}$ & $\stackrel{10}{\rightleftarrows}$ & 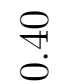 & 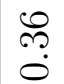 & $\stackrel{\leftrightarrow}{\leftrightarrow}$ \\
\hline$\tilde{\sigma}$ & $\neg$ & $\neg$ & -1 & $\neg$ & $\neg$ & - & - & $\neg$ \\
\hline & & & 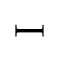 & $\boxminus$ & $\Xi$ & • & $\Xi$ & $\Xi$ \\
\hline & تِ & 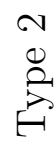 & \multicolumn{3}{|l|}{$\begin{array}{l}\infty \\
0 \\
0 \\
3 \\
0\end{array}$} & \multicolumn{3}{|l|}{ 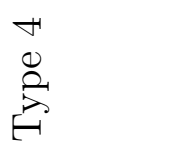 } \\
\hline
\end{tabular}




\begin{tabular}{|c|c|c|c|c|c|c|c|c|c|c|c|c|c|c|c|c|c|}
\hline$\underbrace{\stackrel{\Xi}{g}}_{\tilde{E}}$ & 网 & ๙ి & 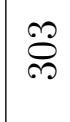 & $\vec{\sigma}$ & 学 & 管 & $\underset{f}{\stackrel{\sigma}{\sigma}}$ & 守 & $\stackrel{9}{\leftrightarrow}$ & $\stackrel{\mathscr{P}}{\mathscr{P}}$ & & 学 & $\begin{array}{l}\text { 婞 } \\
\stackrel{9}{7}\end{array}$ & $\overrightarrow{\stackrel{9}{F}}$ & $\frac{10}{20}$ & 论 & $\stackrel{\infty}{\stackrel{N}{N}}$ \\
\hline $\begin{array}{l}\frac{\pi}{\sigma} \\
\frac{30}{\pi}\end{array}$ & $\stackrel{0}{O}$ & $\stackrel{20}{i d}$ & $\begin{array}{l}0 \\
i\end{array}$ & $\begin{array}{l}20 \\
\stackrel{i}{i}\end{array}$ & $\begin{array}{l}0 \\
\stackrel{i}{i}\end{array}$ & $\stackrel{i}{i}$ & $\begin{array}{l}0 \\
\stackrel{i}{i}\end{array}$ & $\begin{array}{c}0 \\
i\end{array}$ & 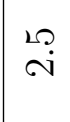 & $\begin{array}{l}\infty \\
i\end{array}$ & & $\vec{\infty}$ & $\stackrel{\infty}{\infty}$ & $\begin{array}{l}10 \\
\mathrm{i}\end{array}$ & $\begin{array}{l}\infty \\
i \\
i\end{array}$ & $\stackrel{+}{i}$ & $\stackrel{\vec{N}}{\vec{N}}$ \\
\hline $0^{10}$ & & & & & & & & & & & & $\stackrel{\mathscr{I}}{\stackrel{0}{0}}$ & $\stackrel{m}{\stackrel{0}{0}}$ & $\vec{m}$ & $\begin{array}{l}\mathscr{O} \\
0 \\
0\end{array}$ & $\stackrel{\sim}{0}$ & $\stackrel{\mathcal{F}}{\breve{0}}$ \\
\hline$\stackrel{10}{20}$ & & & & & & & & & & & & $\begin{array}{l}\infty \\
\infty \\
0 \\
0\end{array}$ & $\begin{array}{l}1 \\
\infty \\
0 \\
0\end{array}$ & $\begin{array}{l}2 \\
\infty \\
0 \\
\dot{0}\end{array}$ & $\stackrel{g}{0}$ & {$\left[\begin{array}{l}\mathscr{2} \\
\infty \\
0\end{array}\right.$} & $\begin{array}{l}\Lambda \\
\infty \\
0\end{array}$ \\
\hline 8 & & & & & & & & & & & & 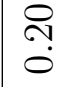 & $\begin{array}{l}\mathscr{O} \\
\stackrel{0}{0}\end{array}$ & $\stackrel{\mathfrak{T}}{\overparen{0}}$ & 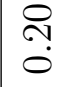 & Ñ & $\underset{\mathfrak{N}}{\tilde{o}}$ \\
\hline$b^{*}$ & & & & $\begin{array}{l}\infty \\
\stackrel{1}{0}\end{array}$ & $\stackrel{H}{\stackrel{H}{0}}$ & $\stackrel{\stackrel{L}{2}}{\stackrel{0}{0}}$ & $\underset{+}{\vec{t}}$ & $\stackrel{10}{\stackrel{10}{0}}$ & $\stackrel{1}{0}$ & 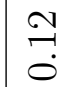 & & $\begin{array}{l}\infty \\
0 \\
0\end{array}$ & $\underset{ָ}{\tilde{O}}$ & $\vec{l}=\vec{c}$ & $\begin{array}{l}\mathscr{O} \\
0 \\
0\end{array}$ & $\overrightarrow{0}$ & $\begin{array}{l}\infty \\
0 \\
0\end{array}$ \\
\hline ¿t & & & & $\begin{array}{l}\infty \\
\infty \\
0 \\
0\end{array}$ & $\begin{array}{l}0 \\
\infty \\
0 \\
\dot{0}\end{array}$ & $\begin{array}{c}\vec{\infty} \\
0 \\
0\end{array}$ & $\begin{array}{l}0 \\
\infty \\
\dot{0}\end{array}$ & $\begin{array}{l}1 \\
1 \\
0\end{array}$ & $\begin{array}{l}\infty \\
\infty \\
0\end{array}$ & $\begin{array}{l}\infty \\
\infty \\
0\end{array}$ & & $\begin{array}{l}\infty \\
1 \\
0\end{array}$ & $\begin{array}{l}\mathscr{b} \\
\dot{0}\end{array}$ & $\begin{array}{l}\mathfrak{P} \\
0\end{array}$ & $\begin{array}{l}1 \\
1 \\
0\end{array}$ & $\begin{array}{l}0 \\
0 \\
0\end{array}$ & $\stackrel{N}{1}$ \\
\hline है & & & & 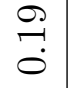 & $\begin{array}{l}\mathscr{\imath} \\
\stackrel{0}{0}\end{array}$ & 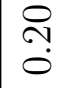 & $\vec{m}$ & $\underset{0}{\not}$ & $\stackrel{\rho}{\rightleftarrows}$ & 命 & & $\stackrel{0}{0}$ & $\stackrel{20}{\stackrel{2}{o}}$ & $\begin{array}{l}\vec{H} \\
0\end{array}$ & 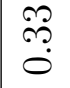 & :̊ & $\begin{array}{l}0 \\
\ddot{0}\end{array}$ \\
\hline$b^{\infty}$ & $\stackrel{\vec{N}}{\stackrel{H}{0}}$ & $\stackrel{\rho}{\stackrel{1}{0}}$ & $\begin{array}{l}0 \\
\ddot{0}\end{array}$ & $\vec{\sharp}$ & $\underset{\dot{c}}{\vec{b}}$ & $\stackrel{m}{\rightleftarrows}$ & $\overrightarrow{7} \cdot \overrightarrow{1}$ & $\stackrel{0}{\stackrel{1}{0}}$ & $\begin{array}{l}8 \\
0\end{array}$ & $\begin{array}{l}8 \\
0 \\
0\end{array}$ & & $\stackrel{\mathfrak{Y}}{\stackrel{0}{0}}$ & $\begin{array}{l}8 \\
0 \\
0\end{array}$ & $\stackrel{m}{\longrightarrow}$ & $\stackrel{1}{0}$ & $\begin{array}{l}1 \\
0 \\
0\end{array}$ & $\begin{array}{l}\infty \\
0 \\
0\end{array}$ \\
\hline$\dddot{\circledast}$ & $\begin{array}{l}\infty \\
\stackrel{\infty}{1} \\
0\end{array}$ & $\vec{\infty}$ & $\begin{array}{l}10 \\
\infty \\
0\end{array}$ & $\begin{array}{l}\ddot{8} \\
\ddot{0} \\
0\end{array}$ & $\begin{array}{l}\infty \\
\infty \\
0 \\
0\end{array}$ & $\overrightarrow{0}$ & 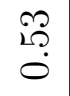 & $\stackrel{19}{\stackrel{19}{0}}$ & $\begin{array}{l}0 \\
0 \\
0\end{array}$ & & & $\stackrel{\infty}{\stackrel{\infty}{0}}$ & $\stackrel{\substack{+0}}{0}$ & $\begin{array}{l}8 \\
0 \\
0\end{array}$ & $\stackrel{n}{\stackrel{1}{0}}$ & $\begin{array}{l}\infty \\
10 \\
0 \\
0\end{array}$ & $\stackrel{\infty}{\stackrel{\infty}{0}}$ \\
\hline 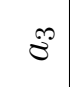 & ĩ & $\stackrel{2 P}{+}$ & $\begin{array}{l}\stackrel{\leftrightarrow}{N} \\
\stackrel{0}{0}\end{array}$ & $\stackrel{\stackrel{\leftrightarrow}{+}}{\circ}$ & $\underset{+}{\stackrel{P}{+}}$ & $\underset{\mathfrak{F}}{\stackrel{f}{0}}$ & $\stackrel{20}{\stackrel{2}{0}}$ & $\underset{0}{\stackrel{H}{0}}$ & 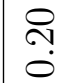 & $\vec{\jmath}$ & & $\begin{array}{l}+\vec{\infty} \\
0 \\
0\end{array}$ & $\underset{:}{\stackrel{H}{0}}$ & $\begin{array}{l}0 \\
\stackrel{1}{0}\end{array}$ & 号 & $\stackrel{10}{\stackrel{0}{0}}$ & $\stackrel{20}{3}$ \\
\hline$\sigma^{N}$ & $\stackrel{0}{0}$ & $\stackrel{1}{\dddot{1}}$ & $\begin{array}{l}0 \\
-1 \\
0\end{array}$ & $\stackrel{\overbrace{}}{\dddot{0}}$ & $\vec{\exists}$ & $\begin{array}{l}\infty \\
0 \\
0\end{array}$ & $\begin{array}{l}\dot{Q} \\
\dot{0} \\
0\end{array}$ & $\dot{O}$ & $\stackrel{10}{\stackrel{10}{0}}$ & $\begin{array}{l}0 \\
0 \\
0\end{array}$ & & $\vec{F}$ & \begin{tabular}{l}
\multirow{2}{O}{} \\
$\stackrel{0}{0}$
\end{tabular} & $\overrightarrow{7} \cdot \overrightarrow{7}$ & $\begin{array}{l}\infty \\
0 \\
0\end{array}$ & $\stackrel{\overbrace{}}{\dddot{O}}$ & $\stackrel{\infty}{\dddot{0}}$ \\
\hline \& & $\begin{array}{c}0 \\
10 \\
0\end{array}$ & $\stackrel{0}{1}$ & 足 & 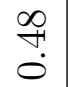 & $\stackrel{\mathscr{P}}{\stackrel{\leftrightarrow}{0}}$ & $\overrightarrow{\tilde{g}}$ & $\stackrel{n}{o}$ & $\stackrel{g}{8}$ & $\stackrel{\vec{H}}{\overrightarrow{0}}$ & 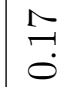 & & 告 & $\stackrel{1}{\circ}$ & 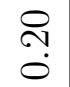 & ֶి & $\stackrel{m}{\stackrel{0}{0}}$ & $\stackrel{m}{\rightleftarrows}$ \\
\hline $\mathfrak{S}$ & $\overrightarrow{0}$ & $\stackrel{\sim}{\circ}$ & $\stackrel{8}{0}$ & $\begin{array}{l}\vec{b} \\
0 \\
0\end{array}$ & $\stackrel{\infty}{\stackrel{\infty}{0}}$ & $\vec{c}$ & $\begin{array}{l}\mathscr{\imath} \\
\stackrel{0}{0}\end{array}$ & $\stackrel{10}{\stackrel{10}{0}}$ & $\stackrel{+}{\stackrel{+}{0}}$ & 号 & & ชి & $\overrightarrow{7}$ & $\begin{array}{l}\stackrel{\Re}{\vartheta} \\
\stackrel{0}{0}\end{array}$ & 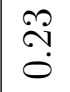 & $\stackrel{R}{R}$ & 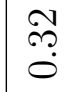 \\
\hline$\vec{b}$ & $\vec{H}$ & $\stackrel{\infty}{\stackrel{\infty}{0}}$ & $\begin{array}{l}0 \\
\dddot{0}\end{array}$ & $\begin{array}{l}0 \\
\stackrel{1}{0}\end{array}$ & $\stackrel{20}{\longrightarrow}$ & $\stackrel{10}{\rightleftarrows}$ & $\underset{\vec{c}}{\overrightarrow{0}}$ & $\stackrel{\mathfrak{Y}}{\dddot{0}}$ & $\stackrel{\vec{H}}{\overrightarrow{0}}$ & $\stackrel{m}{\dddot{0}}$ & & $\vec{F}$ & $\begin{array}{l}8 \\
0 \\
0\end{array}$ & $\overrightarrow{7} \cdot \overrightarrow{7}$ & $\overrightarrow{7}$ & $\stackrel{m}{\stackrel{0}{0}}$ & $\underset{\not}{\rightleftarrows}$ \\
\hline$\vec{\phi}$ & $\stackrel{0}{0}$ & $\stackrel{\sim}{\stackrel{2}{0}}$ & 解 & $\stackrel{\oplus}{\overrightarrow{0}}$ & $\begin{array}{l}0 \\
\stackrel{0}{0}\end{array}$ & $\stackrel{0}{0}$ & $\stackrel{\sim}{\breve{0}}$ & ְ̊ & $\stackrel{\infty}{+}$ & : & & $\stackrel{\infty}{\stackrel{\infty}{0}}$ & $\stackrel{\mathscr{a}}{\stackrel{0}{0}}$ & $\begin{array}{l}\stackrel{\Re}{?} \\
\stackrel{0}{0}\end{array}$ & $\vec{H}$ & $\stackrel{\Re}{\stackrel{\leftrightarrow}{0}}$ & ஜ̊ \\
\hline$\vec{\sigma}$ & $\neg$ & - & $\neg$ & -1 & $\neg$ & $\neg$ & $\neg$ & $\neg$ & -1 & - & & - & - & $\neg$ & $\neg$ & $-r$ & -1 \\
\hline$\sum_{n}^{\infty}$ & $\stackrel{N}{I}$ & $\vec{\Lambda}$ & $\stackrel{\check{I}}{\perp}$ & 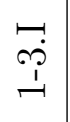 & $\underset{I}{\stackrel{H}{I}}$ & ป̇ & $\underset{\infty}{\vec{I}}$ & $\underset{F}{\vec{I}}$ & 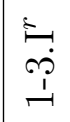 & ì & 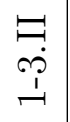 & $\underset{+}{\stackrel{\Xi}{+}}$ & $\underset{\stackrel{P}{d}}{\vec{d}}$ & $\begin{array}{l}\vec{H} \\
\stackrel{1}{d}\end{array}$ & 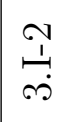 & 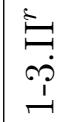 & 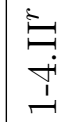 \\
\hline
\end{tabular}




\section{Appendix B}

\section{Data Pre-processing Code}

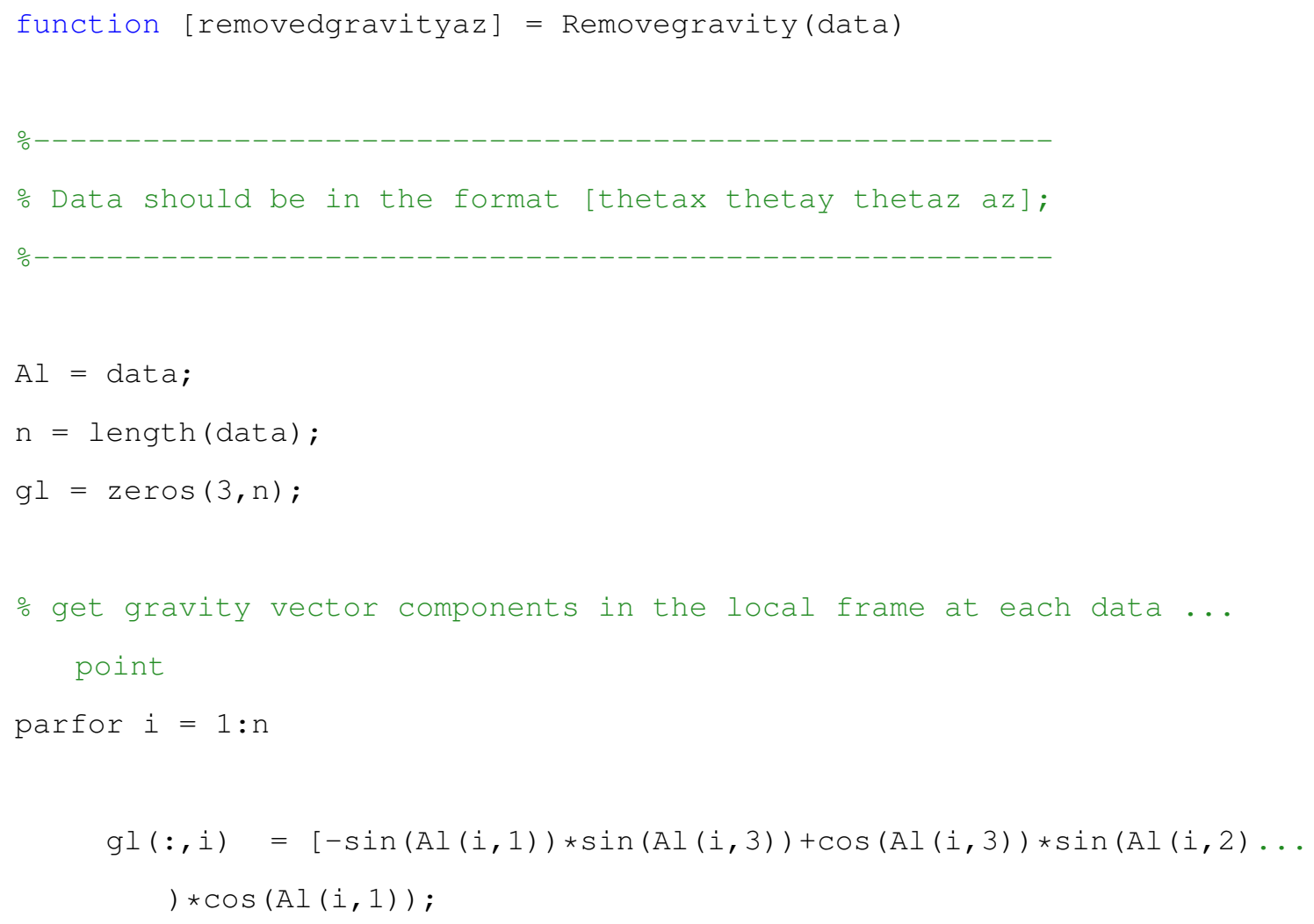




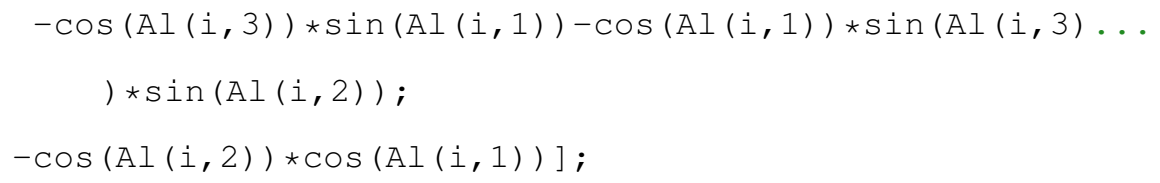

end

a add components to acceleration

for $i=1: n$

$A \perp(i, 4)=A l(i, 4)+g l(3, i) ;$

end

removedgravityaz $=A l(:, 4)$;

end

function [peak] = Extractpeaks (time, acceleration, treshold)

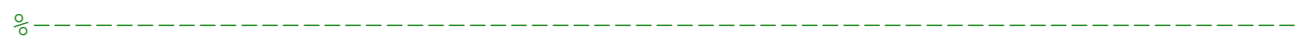

- Pulses are extracted from the data (time, acceleration) based

$\div$ on the specified treshold. The pulses are stored in the

ostructure peak. peak contains 5 fields:

o peak.az(n) values $(:)$ = acceleration values of pulse $n$

\% peak.time(n).values(:) = time values of pulse $n$

\% peak.aMax $(n)=$ maximum acceleration of pulse $n$

\% peak.duration( $\mathrm{n})$ = duration of pulse $\mathrm{n}$

o peak.durationtomax $(n)=$ peak time of pulse $n$

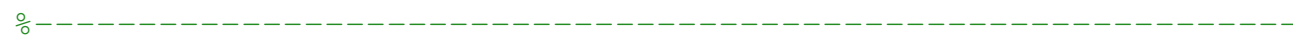

$\mathrm{az}=$ acceleration;

$\mathrm{n}=$ length (acceleration) -1000 ; 


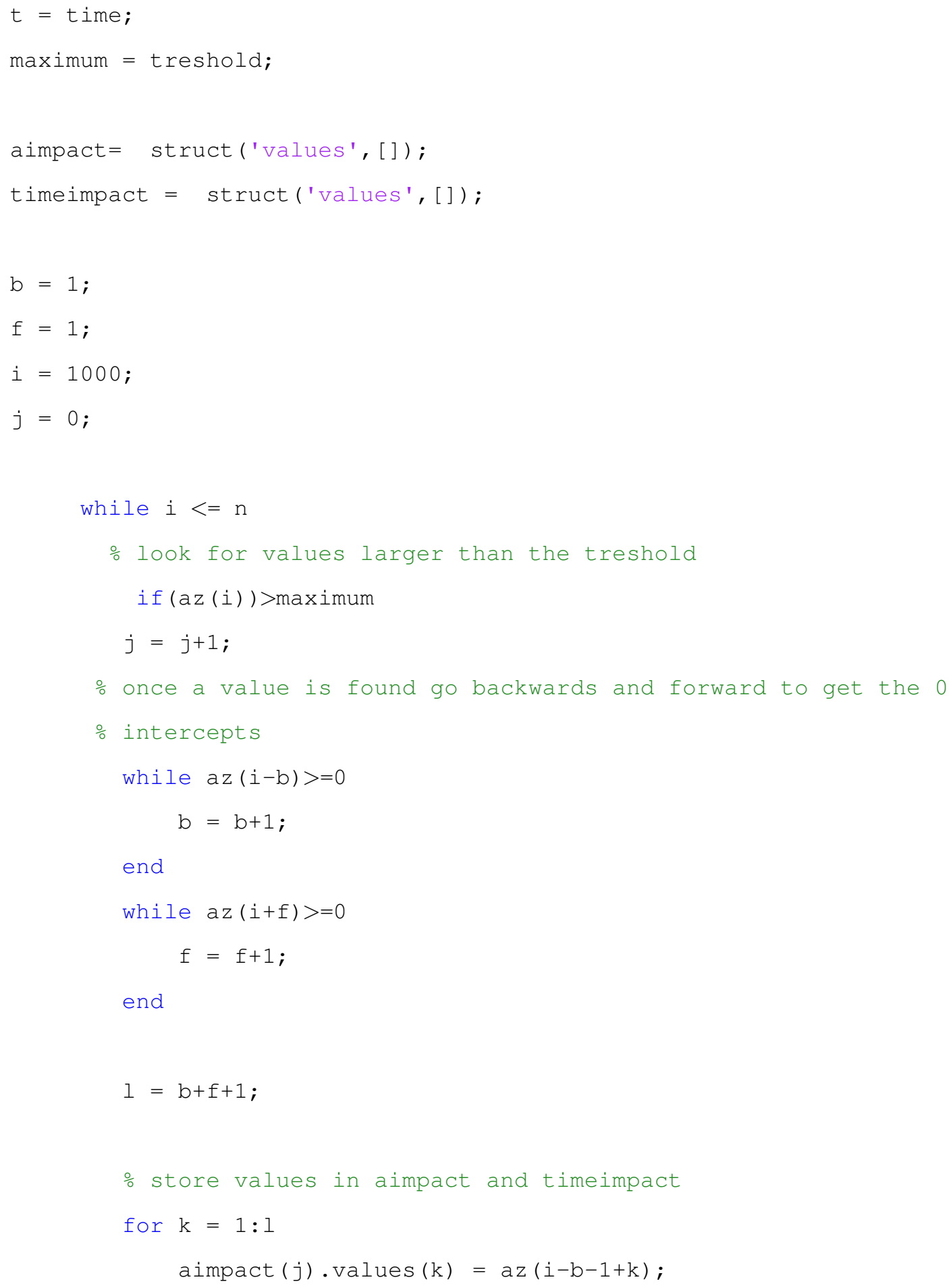




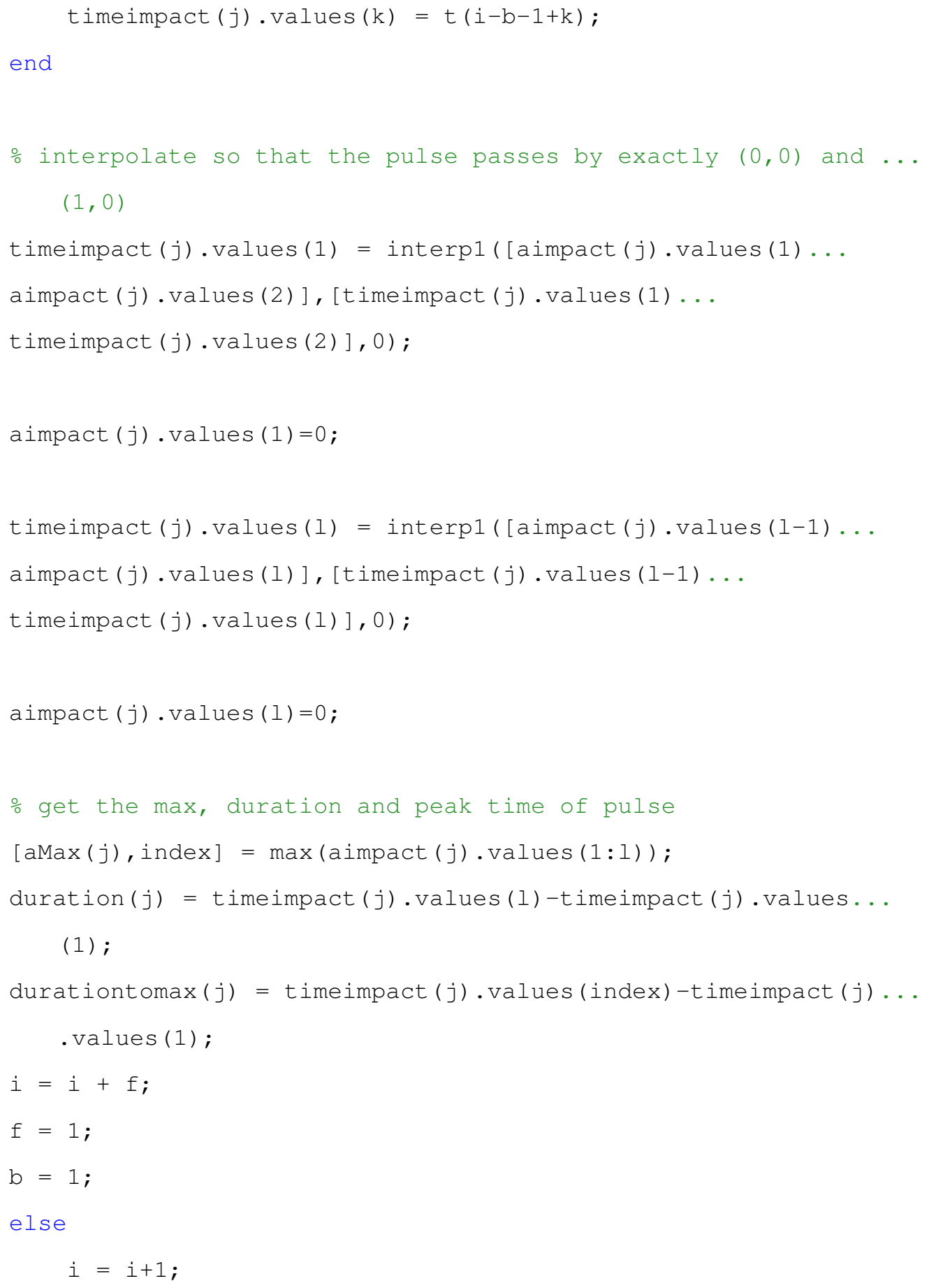


end

end

\% store everything in the structure peak

peak = struct ('az', aimpact, 'time', timeimpact, 'aMax', aMax, ...

'duration', duration, 'durationtomax', durationtomax) ;

end 


\section{Appendix C}

\section{Curve Fitting Algorithm}

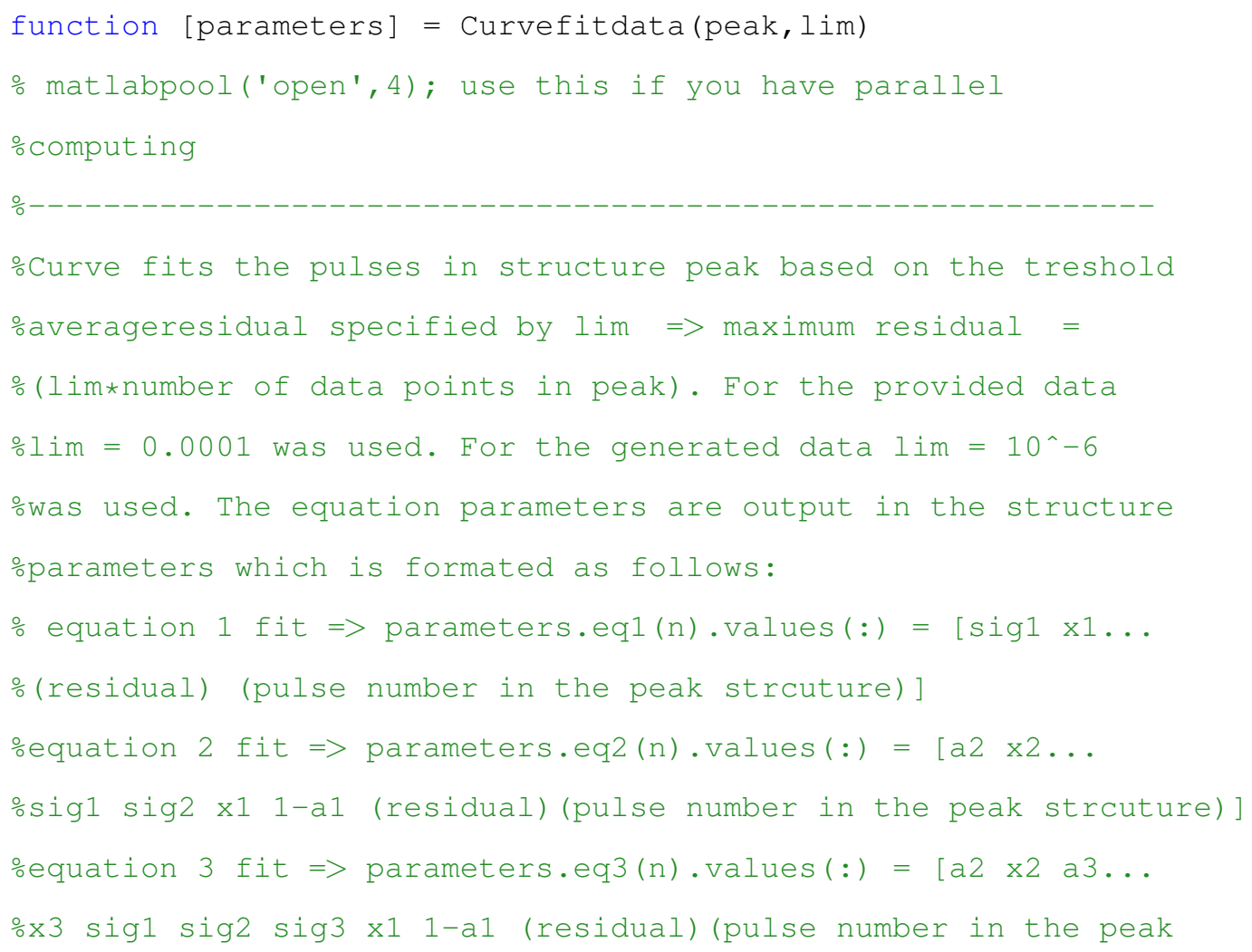




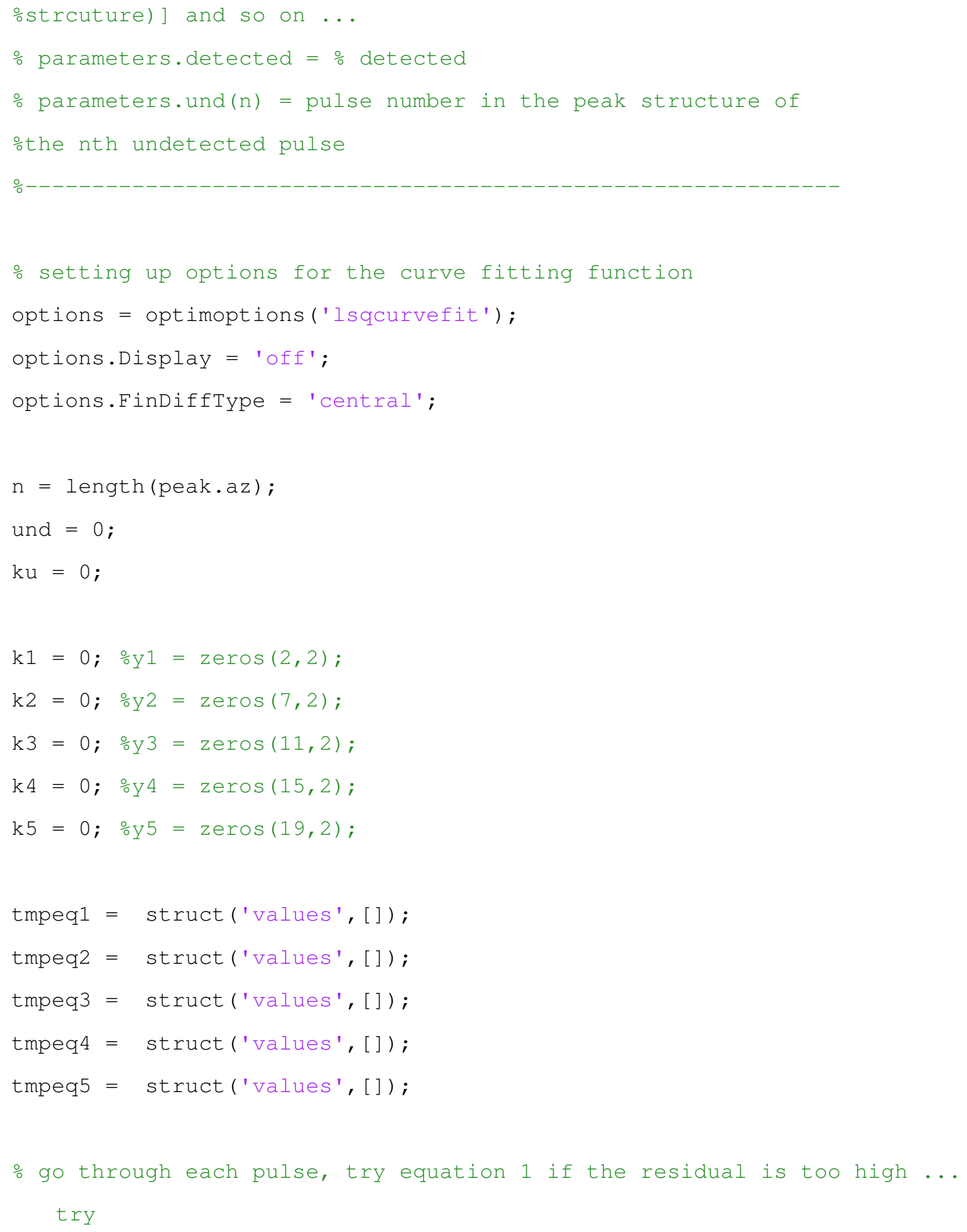




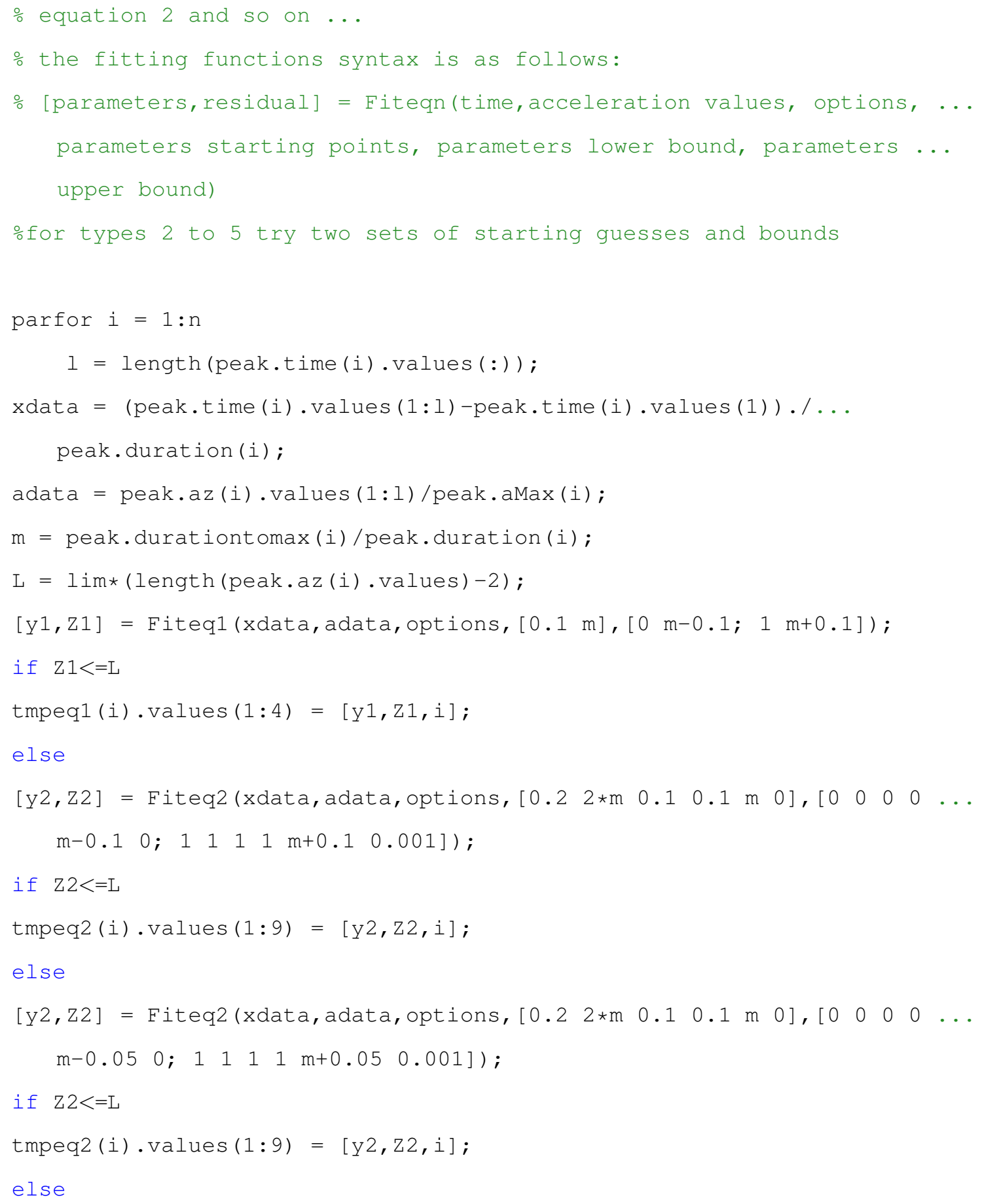




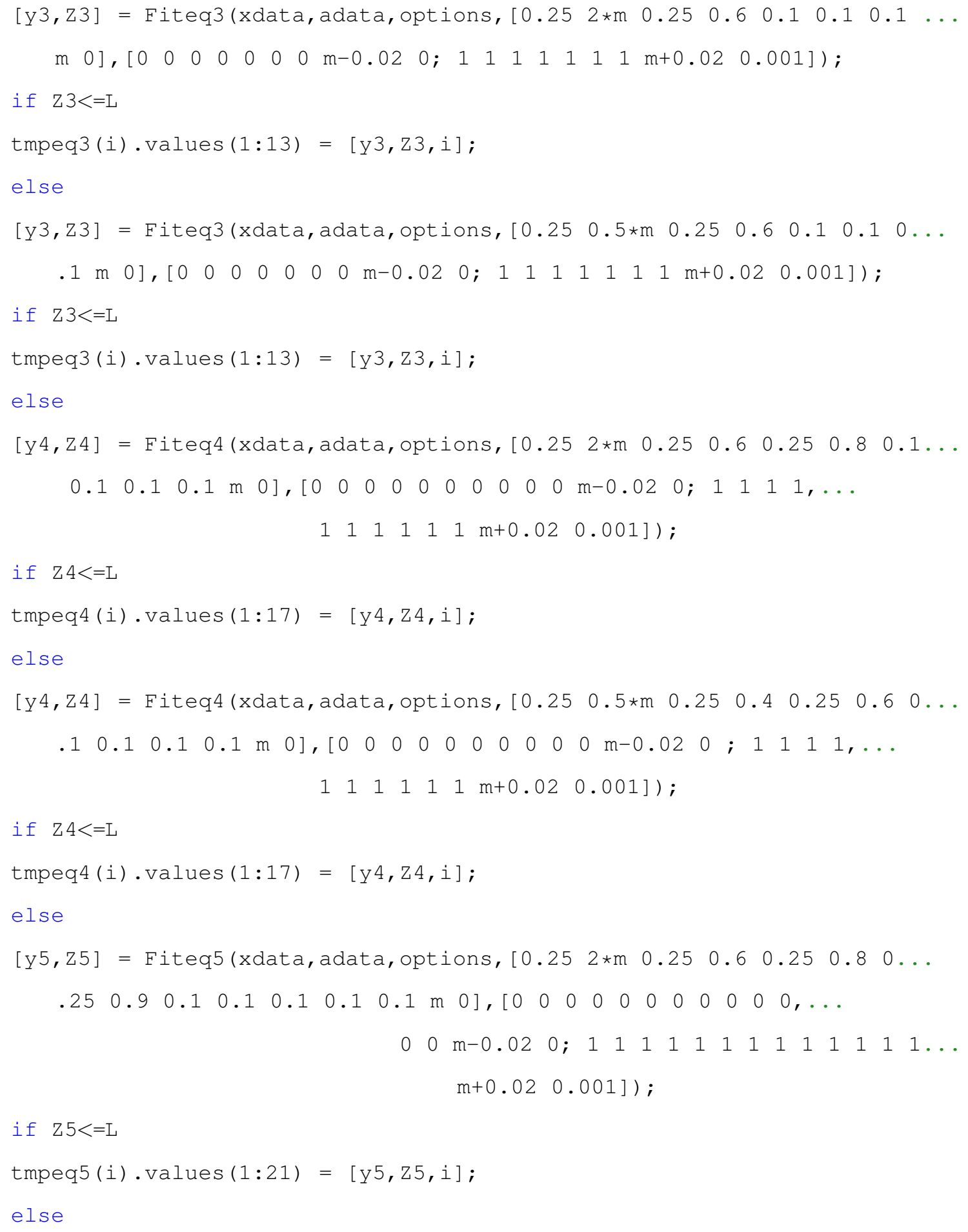




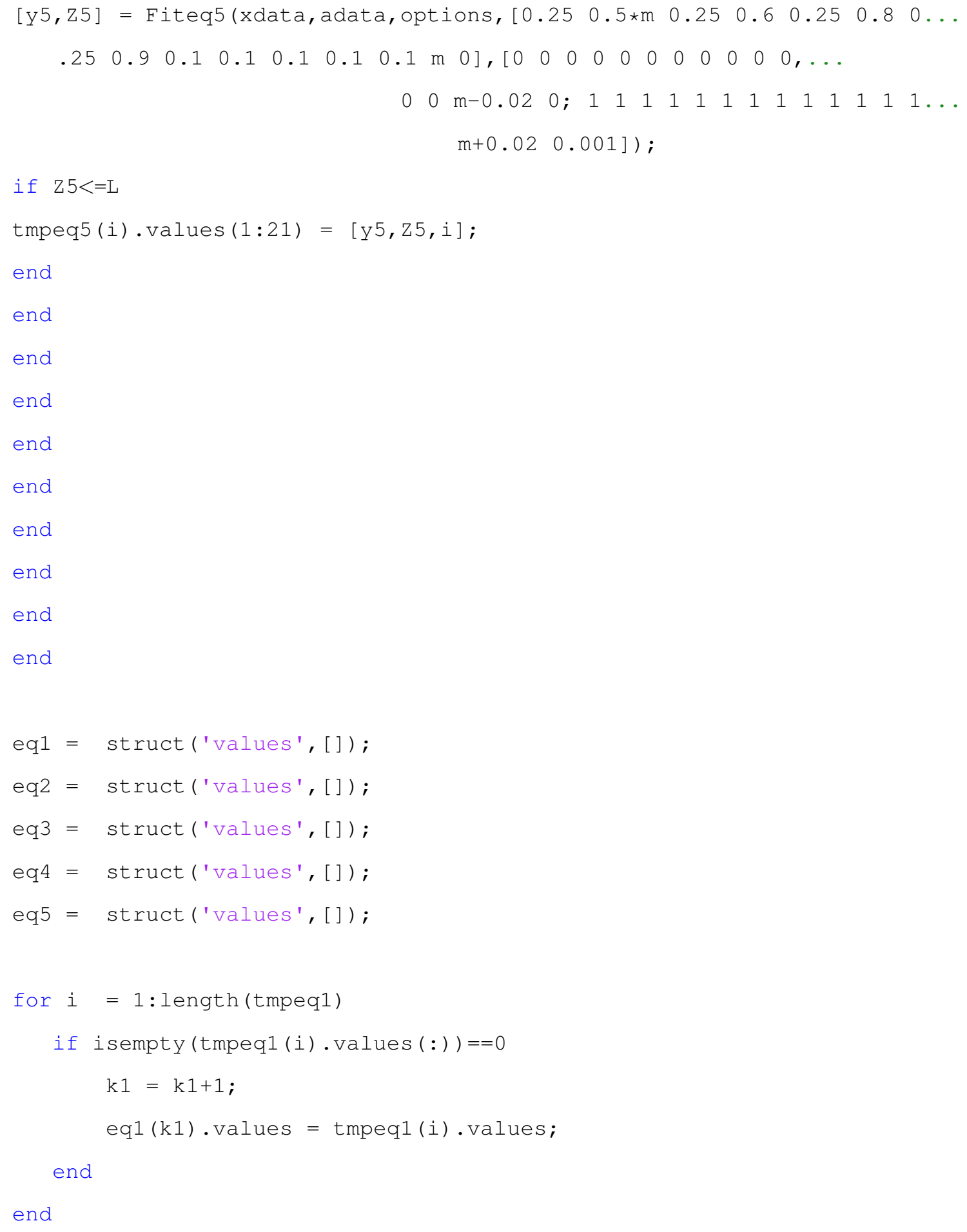




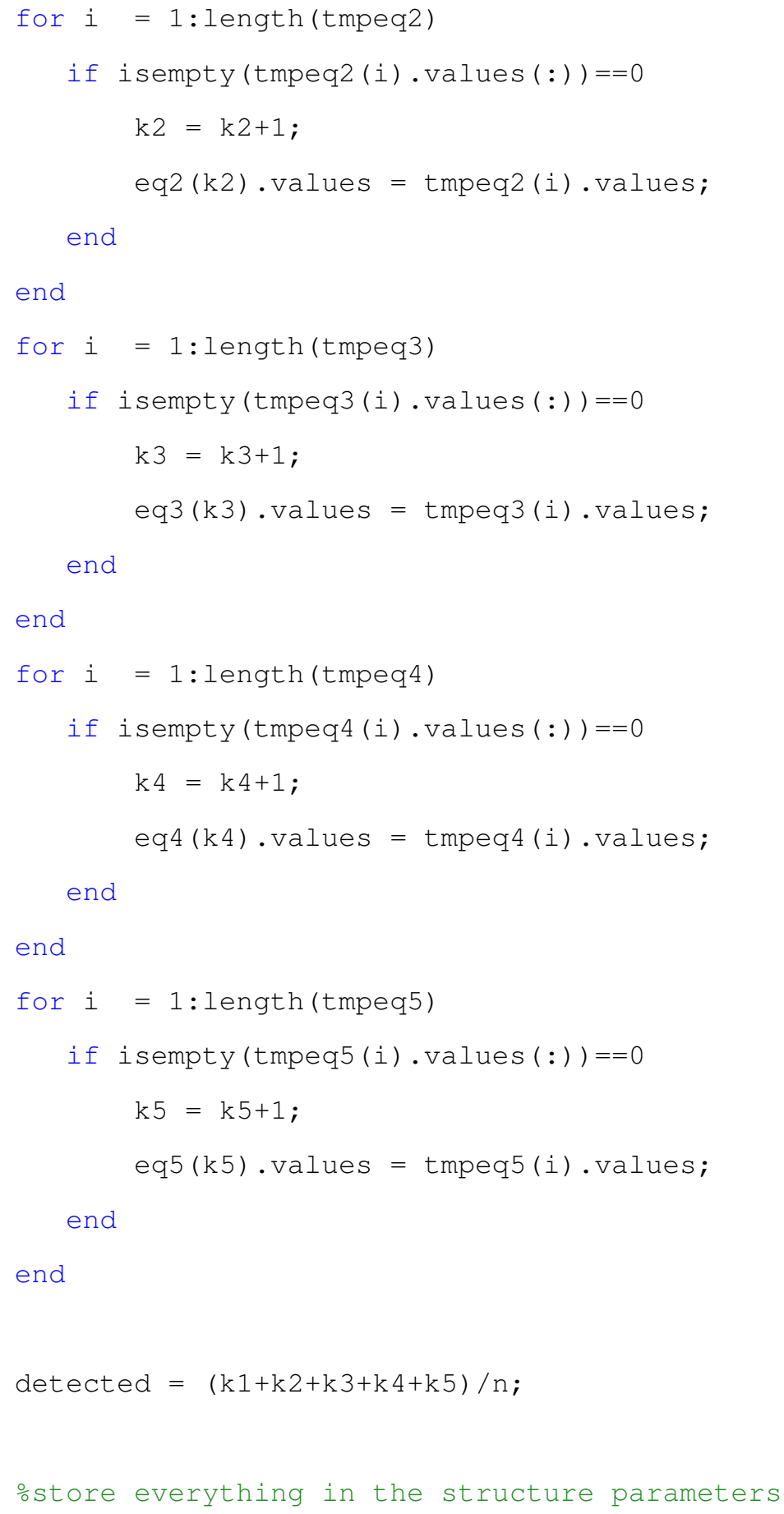




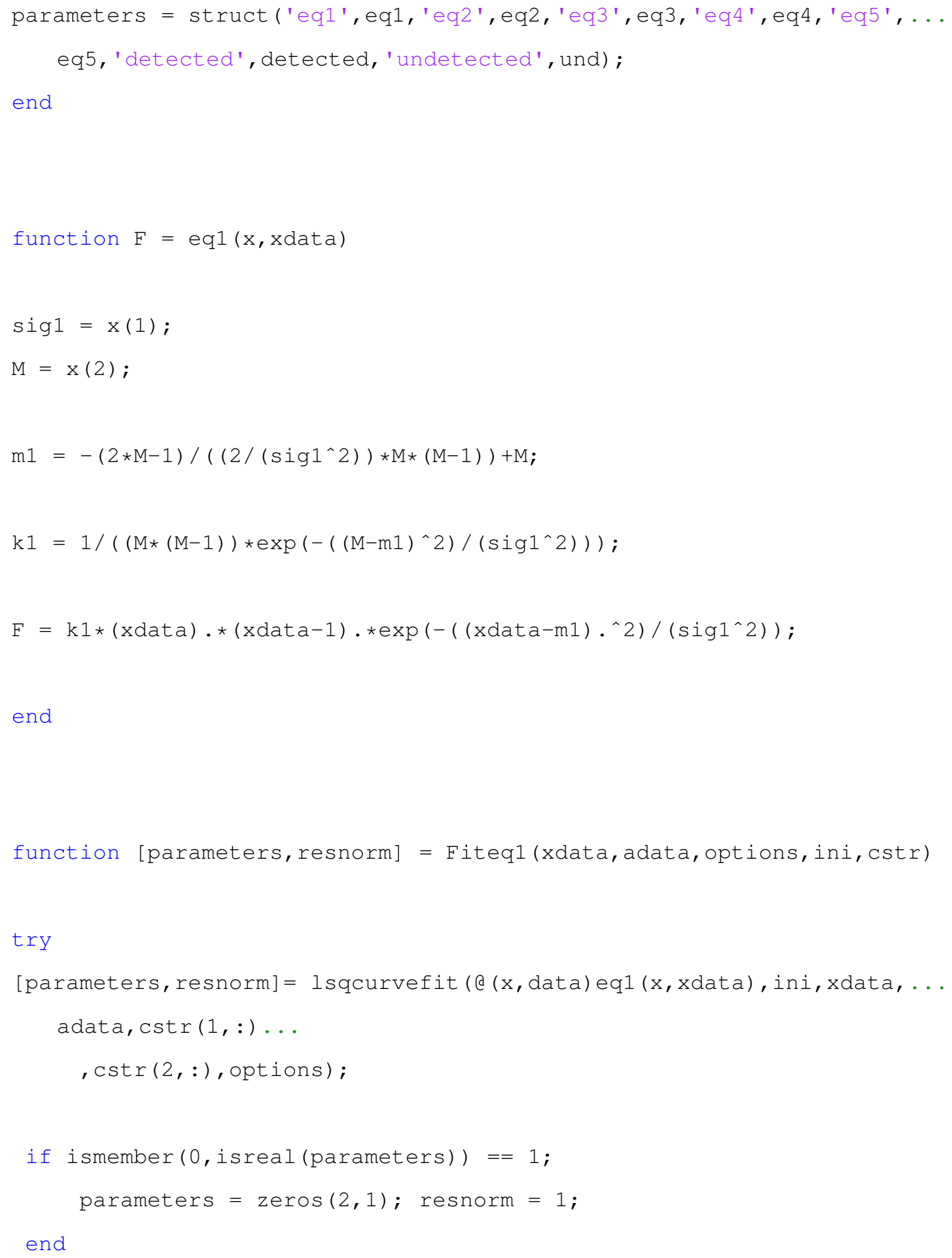




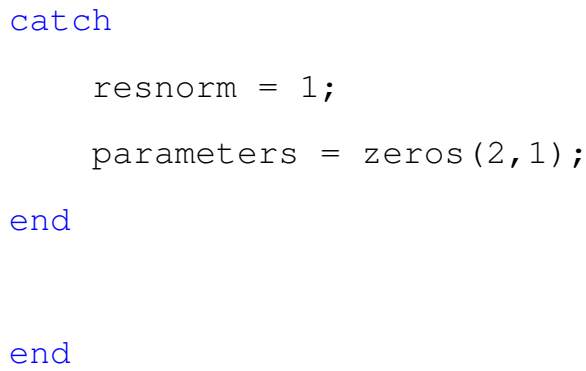




\section{Appendix D}

\section{Classification of Fitted Pulses into the Different Profiles Code}

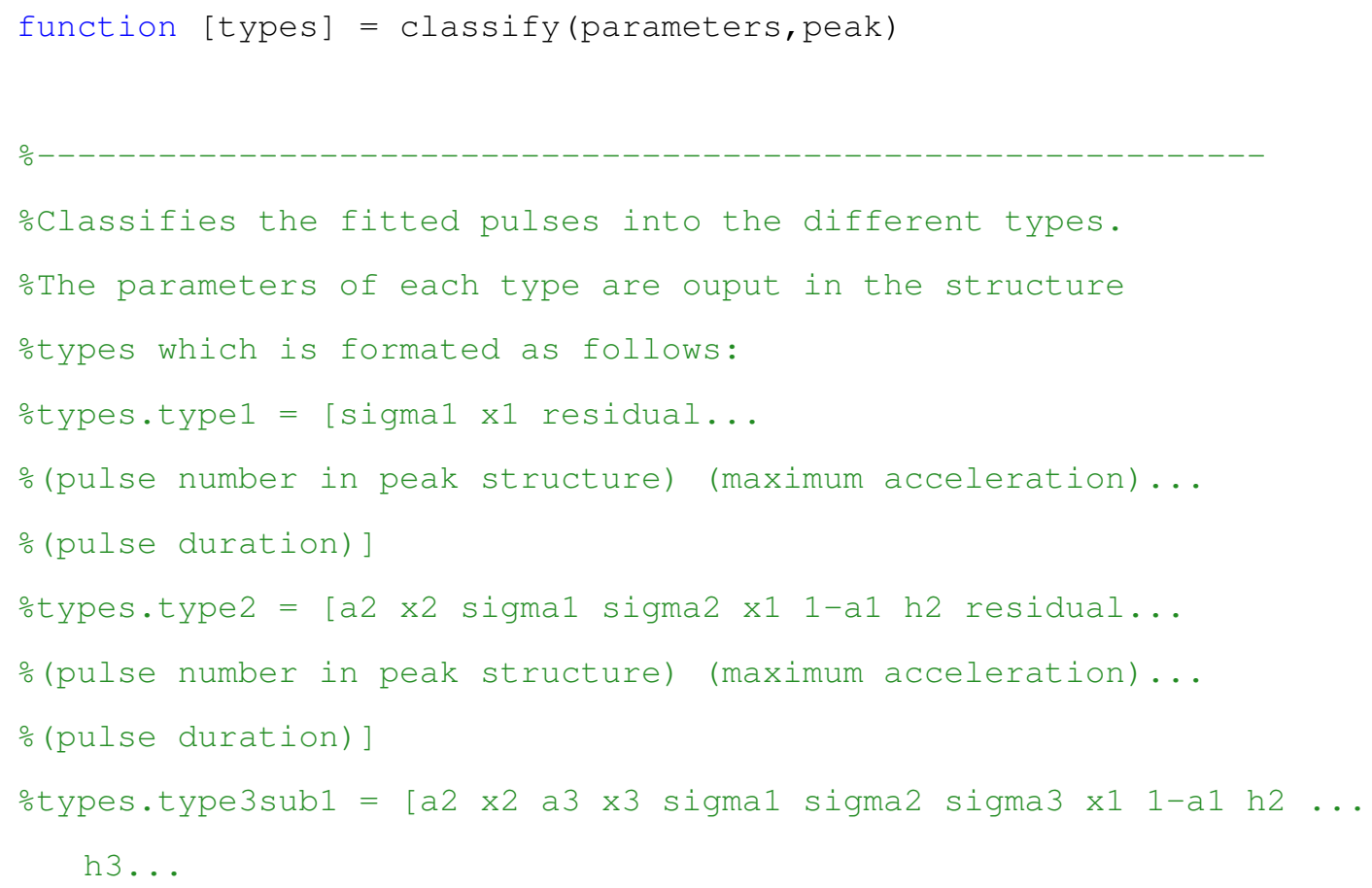




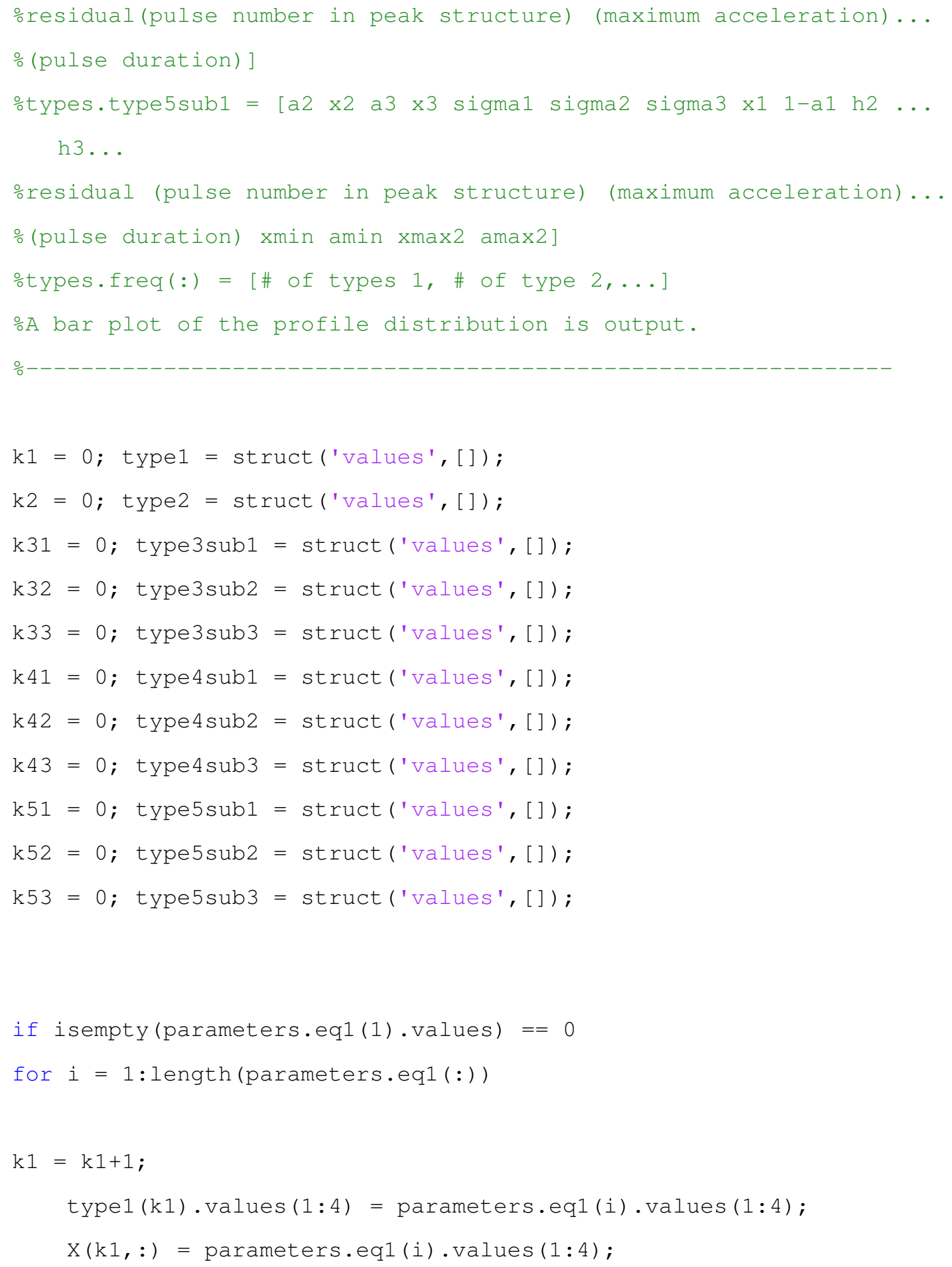




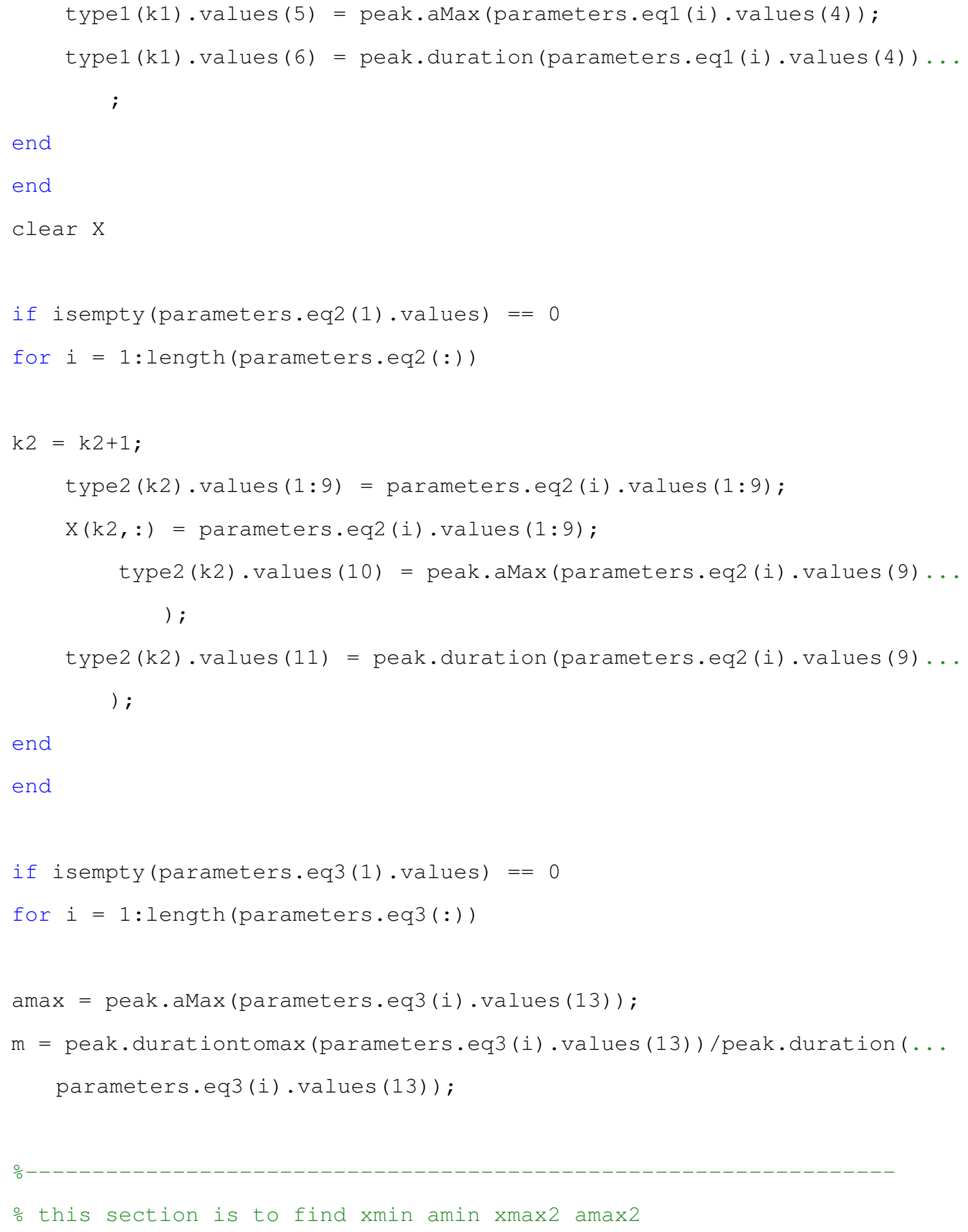


$\mathrm{x} 1$ = linspace (parameters.eq3 (i).values (8), parameters.eq3 (i) . values...

(2), 100);

y1 = eq3 (parameters.eq3 (i). values $(1: 9), x 1)$;

$[\min 1, \operatorname{ind} 1]=\min (y 1)$;

if parameters.eq3 (i).values (8)<parameters.eq3 (i) . values (2)

$\mathrm{xp} 1=$ linspace $(\mathrm{x} 1($ ind 1$), 1,100)$;

else

$\operatorname{xp} 1=1$ inspace $(x 1($ ind 1$), 0,100)$;

end

yp1 = eq3 (parameters.eq3 $(i) \cdot \operatorname{values}(1: 9), \operatorname{xp} 1) ;$

$[\max 1, \mathrm{indm} 1]=\max (\operatorname{yp} 1)$;

$\mathrm{H}(1,:)=[\mathrm{x} 1(\mathrm{ind} 1) \min 1 \mathrm{xp} 1(\mathrm{indm} 1) \max 1]$;

x2 = linspace(parameters.eq3(i).values(8), parameters.eq3(i) . values...

(4), 100);

$y^{2}=$ eq3 (parameters.eq3 (i). $\left.\operatorname{values}(1: 9), x 2\right)$;

$[\min 2, \mathrm{ind} 2]=\min \left(\mathrm{y}^{2}\right) ;$

if parameters.eq3 (i) . values (8)<parameters.eq3 (i) . values (4)

xp2 = linspace $($ x2 (ind2), 1, 100);

else

xp2 = linspace $(x 2($ ind 2$), 0,100) ;$

end

yp2 = eq3 (parameters.eq3 (i) $\operatorname{values}(1: 9), \operatorname{xp} 2)$;

$[\max 2, \mathrm{indm} 2]=\max (\mathrm{yp} 2)$;

$\mathrm{H}(2, \mathbf{)})=[\mathrm{x} 2(\mathrm{ind} 2) \min 2 \mathrm{xp} 2(\mathrm{indm} 2) \max 2]$;

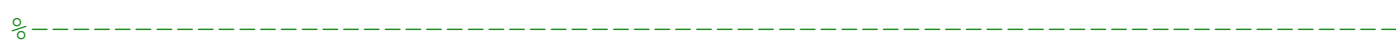

if $\max ([(\max 1-\min 1),(\max 2-\min 2)])>0.2$

$\mathrm{k} 51=\mathrm{k} 51+1$;

type5sub1 (k51).values (1:13) = parameters.eq3(i).values(1:13); 


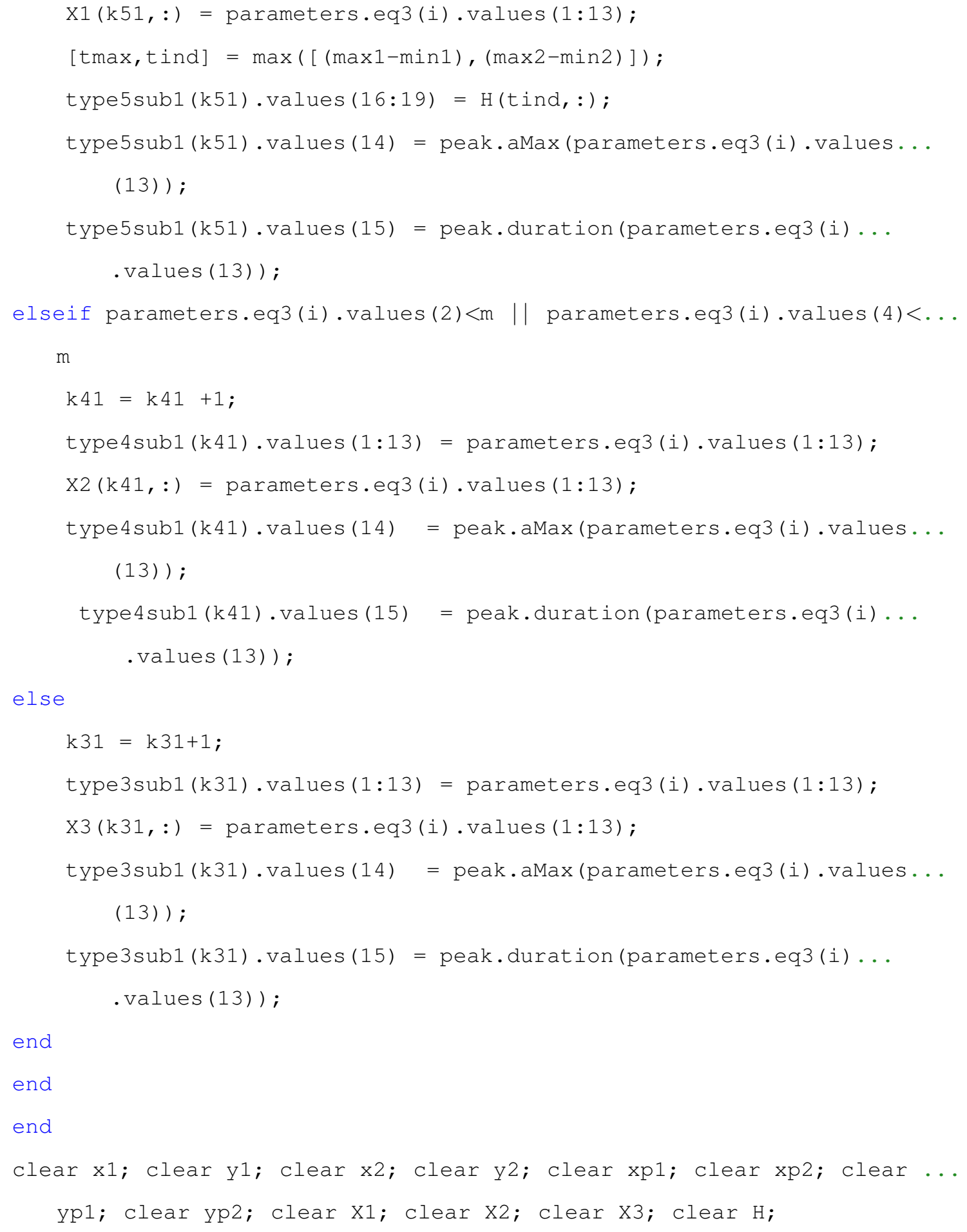




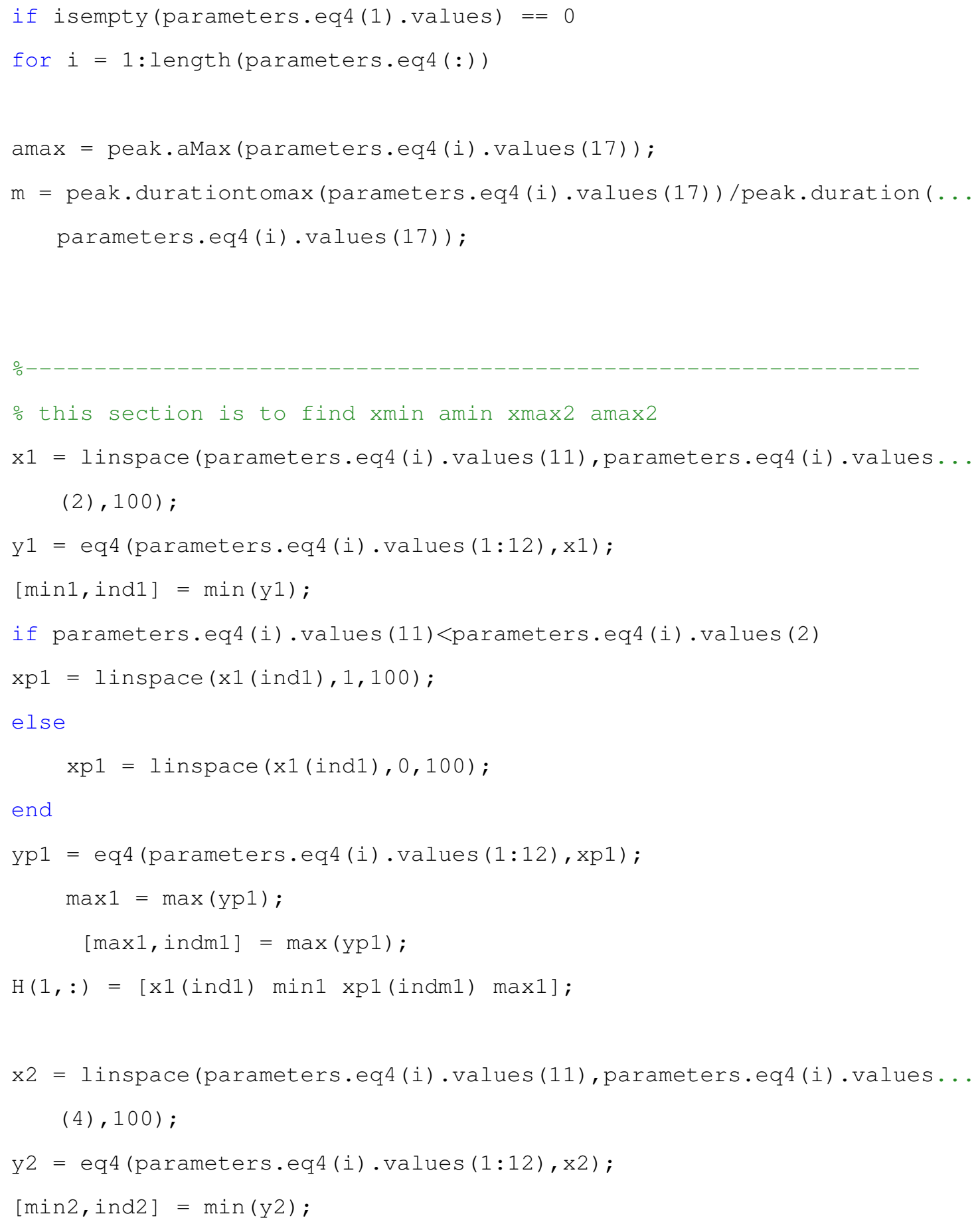




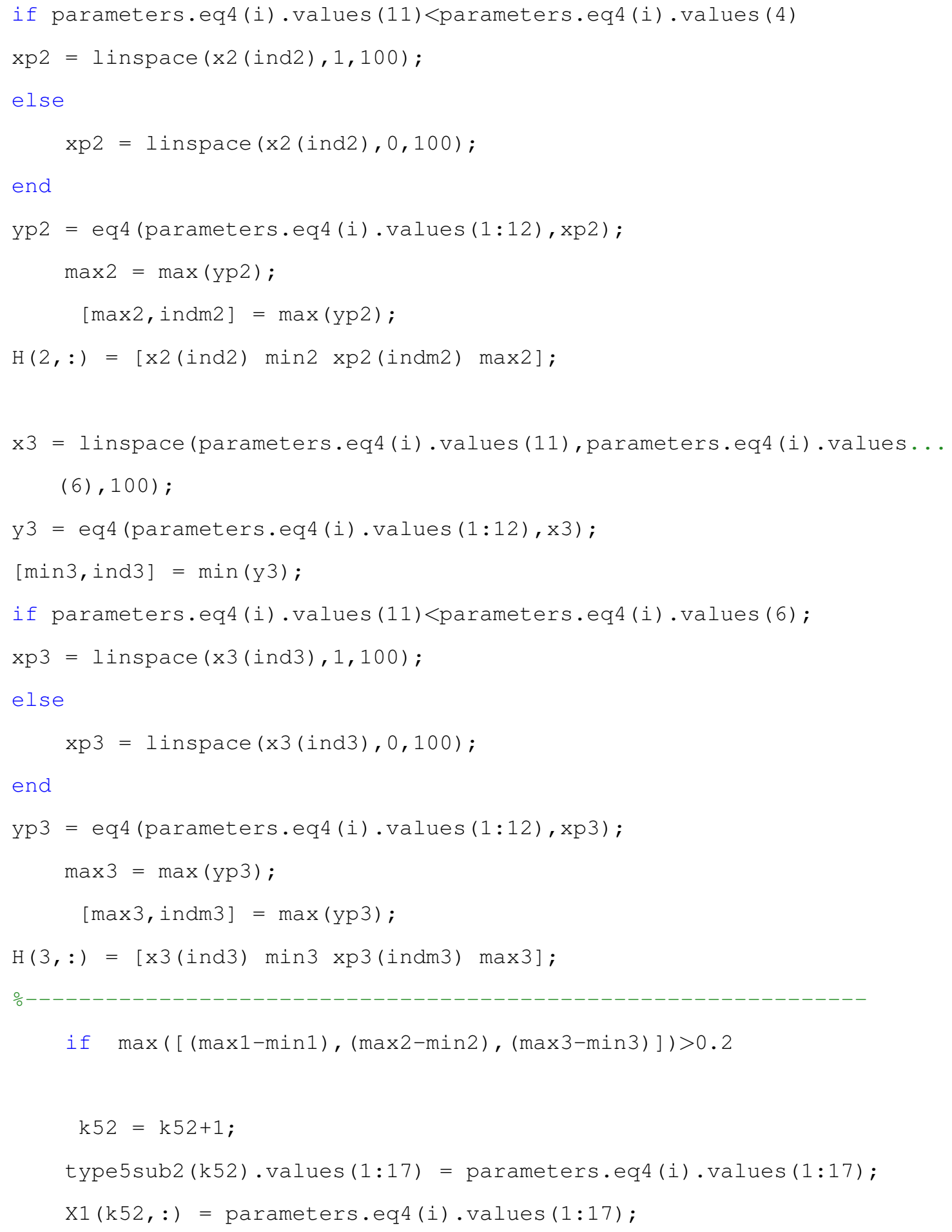




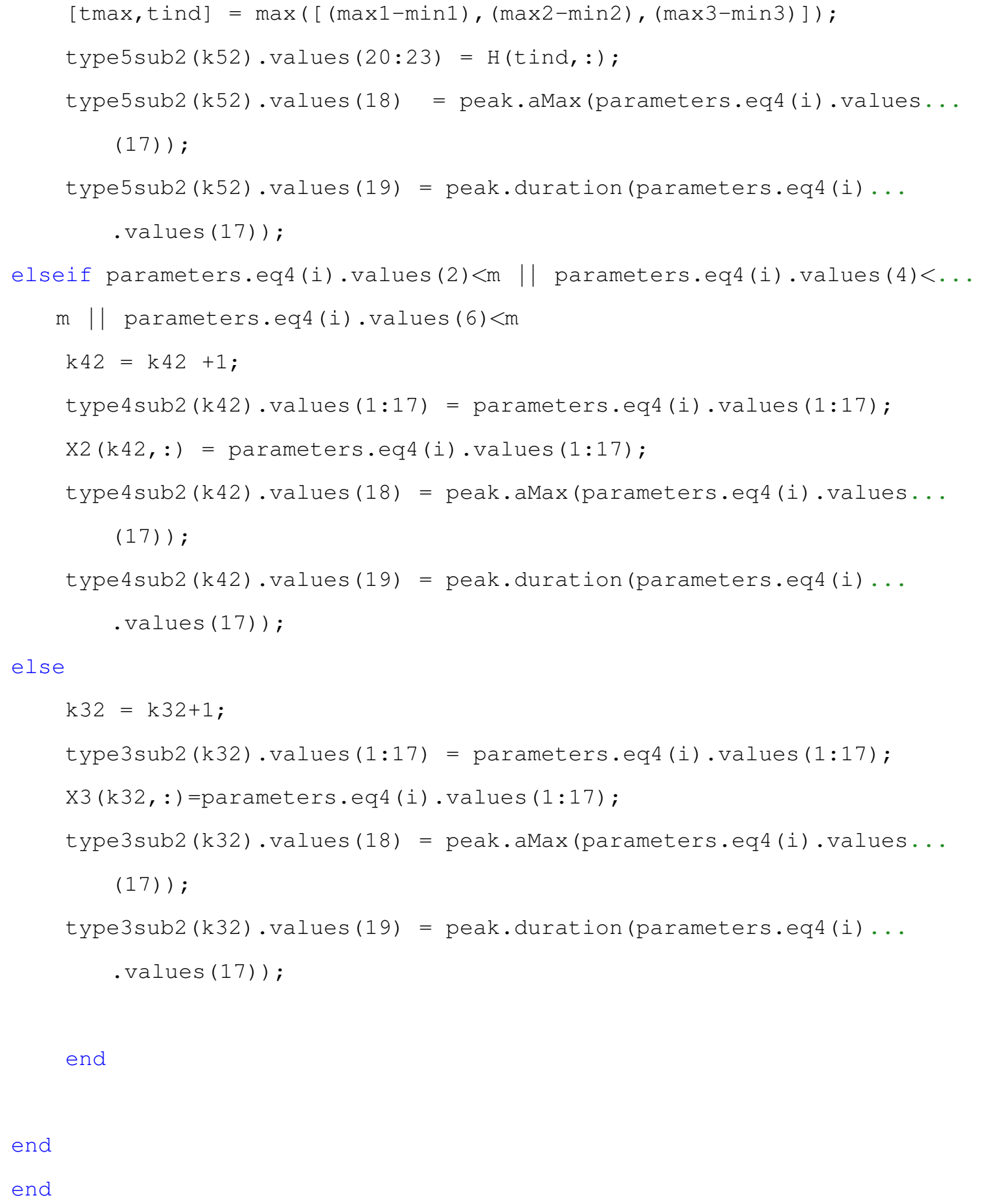




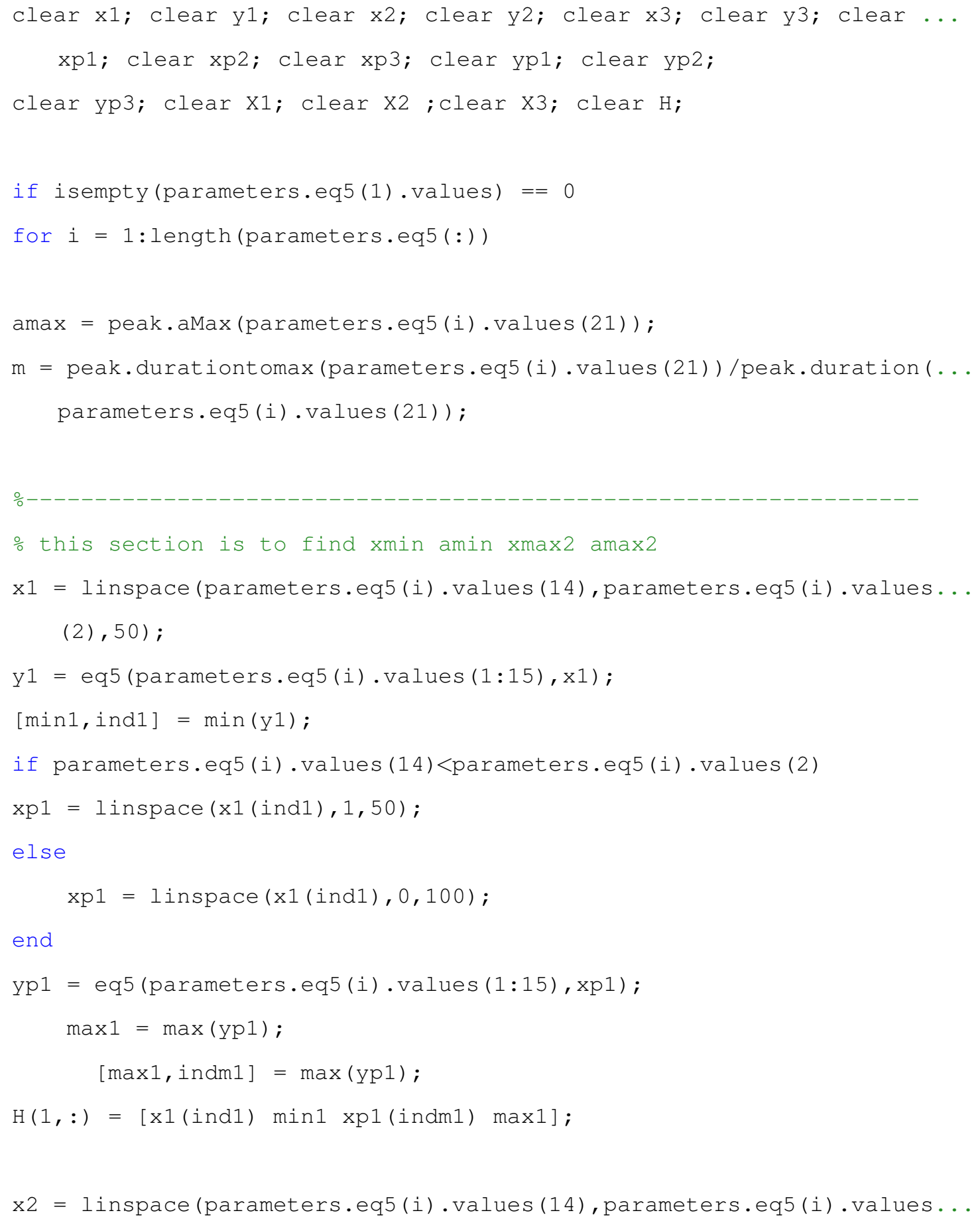




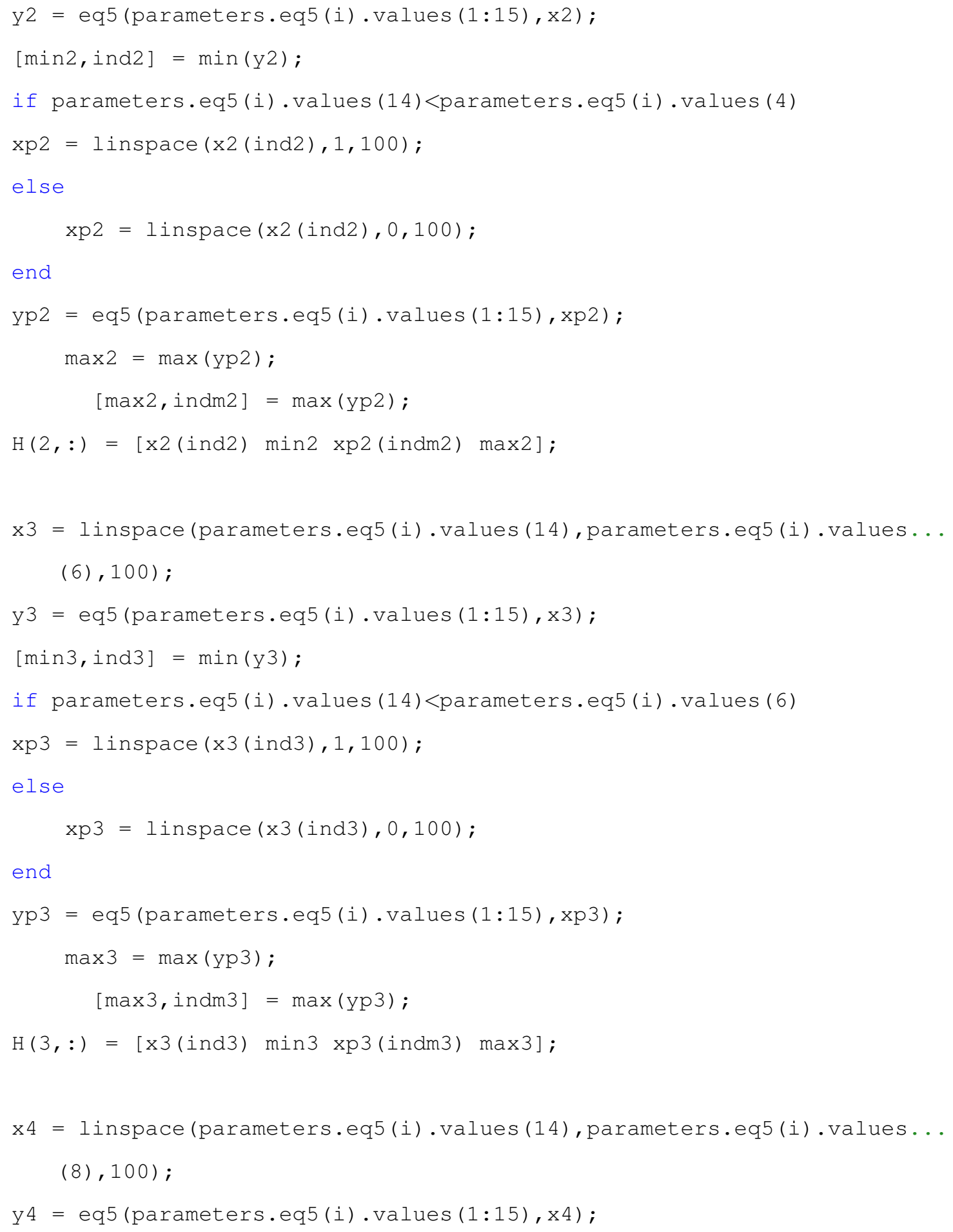




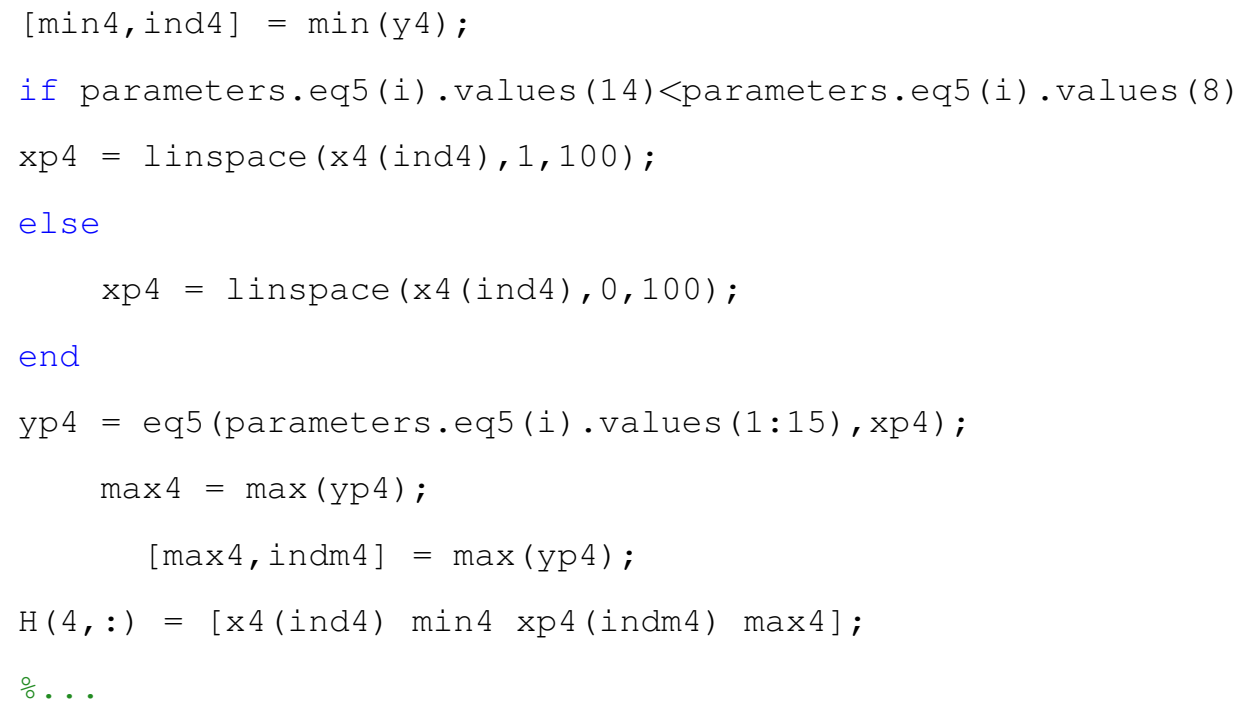




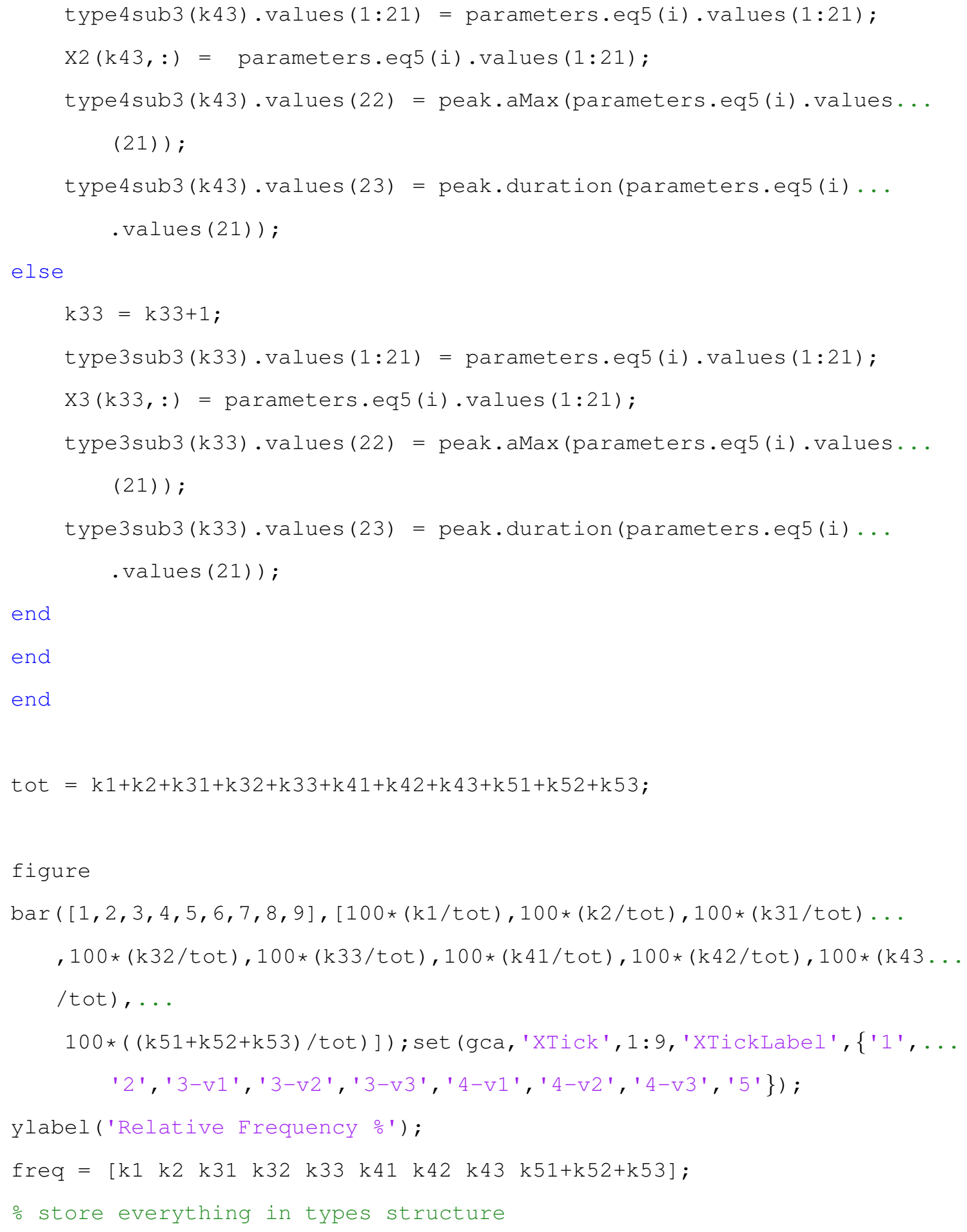




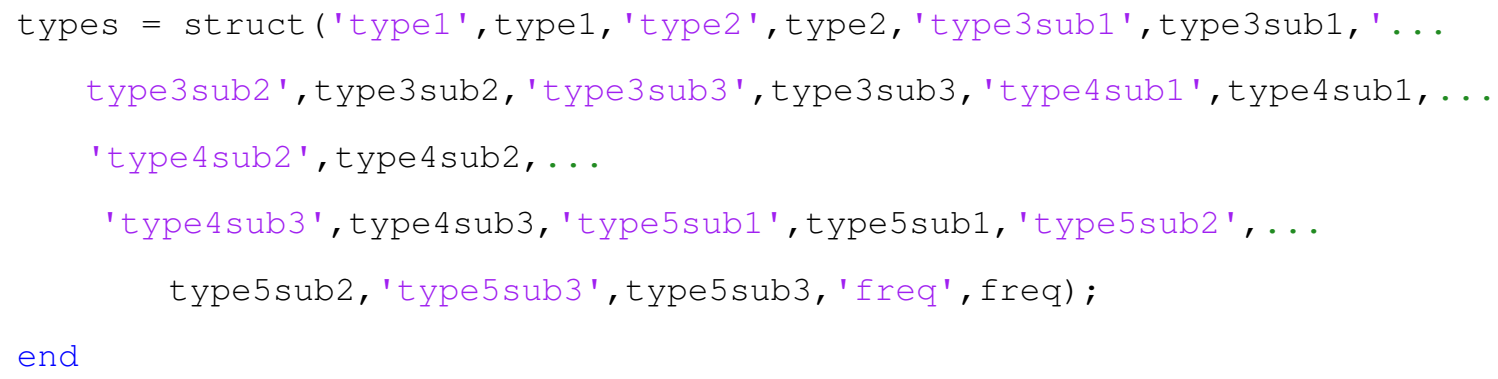




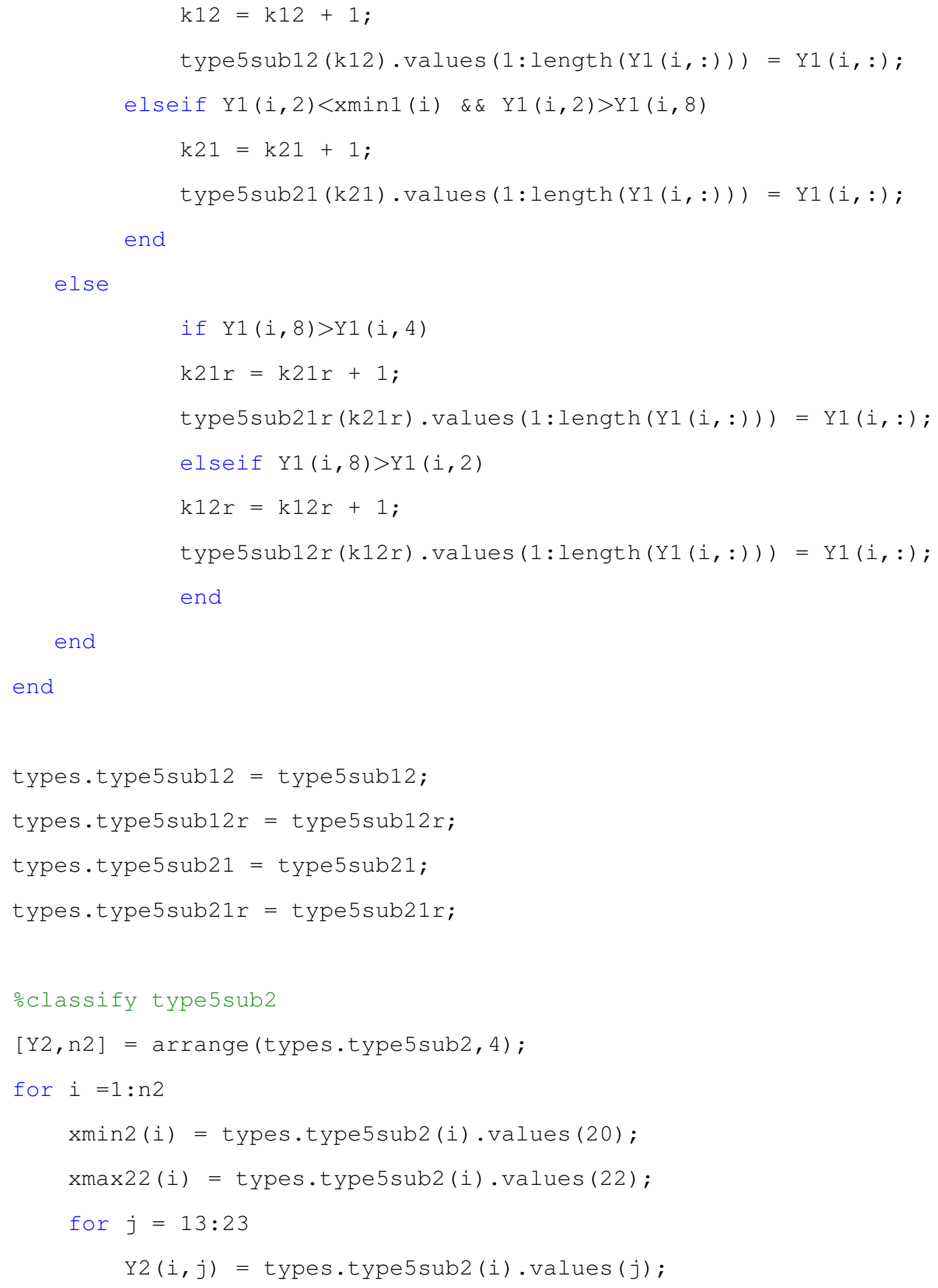




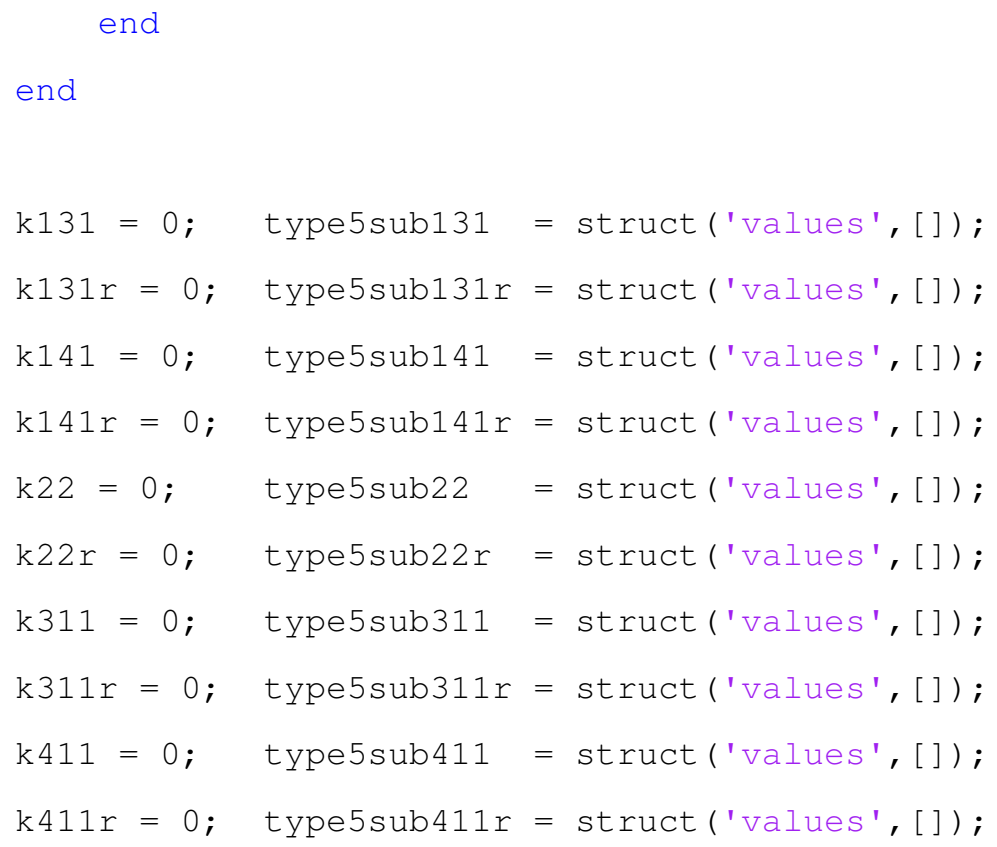




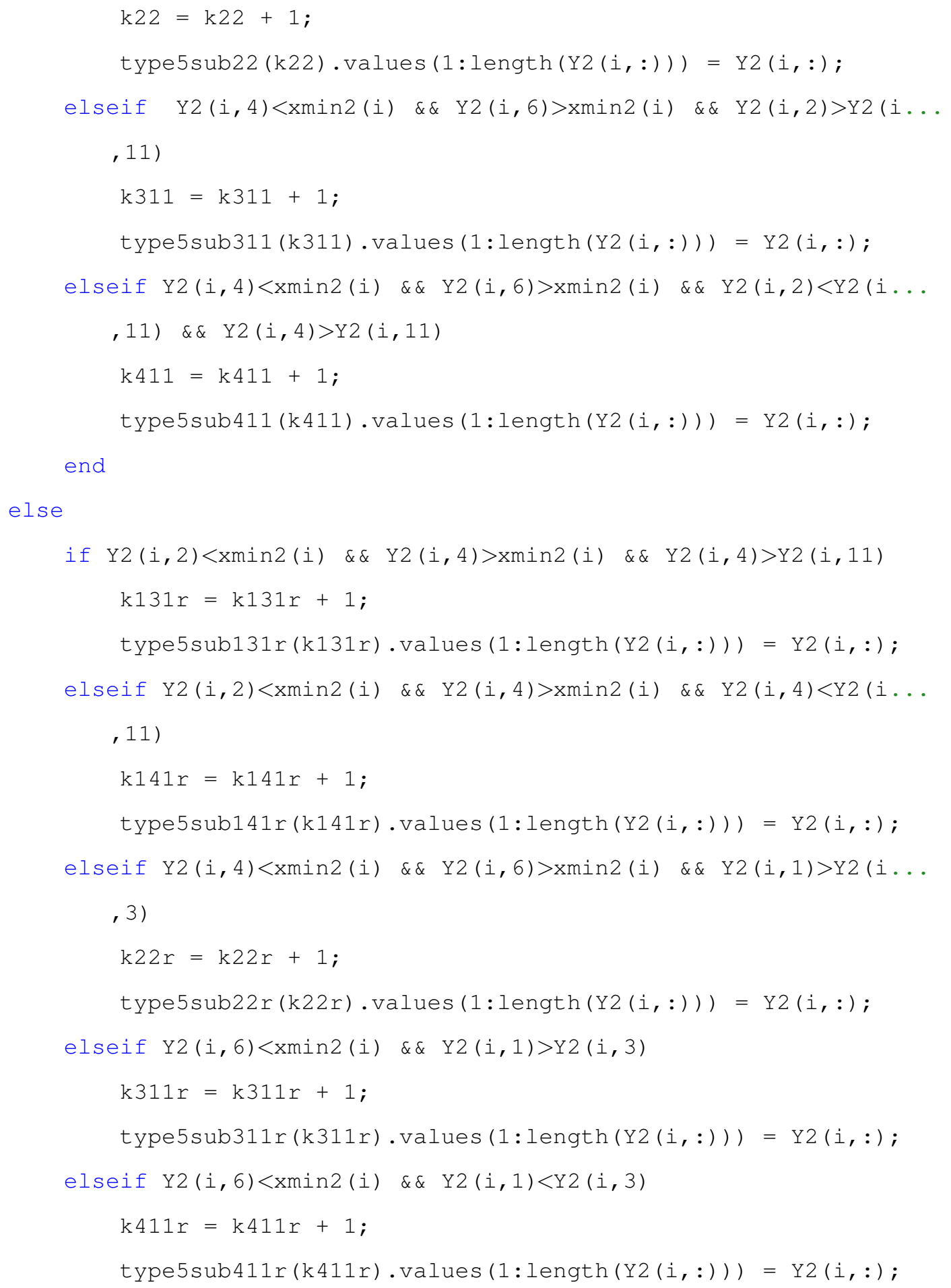




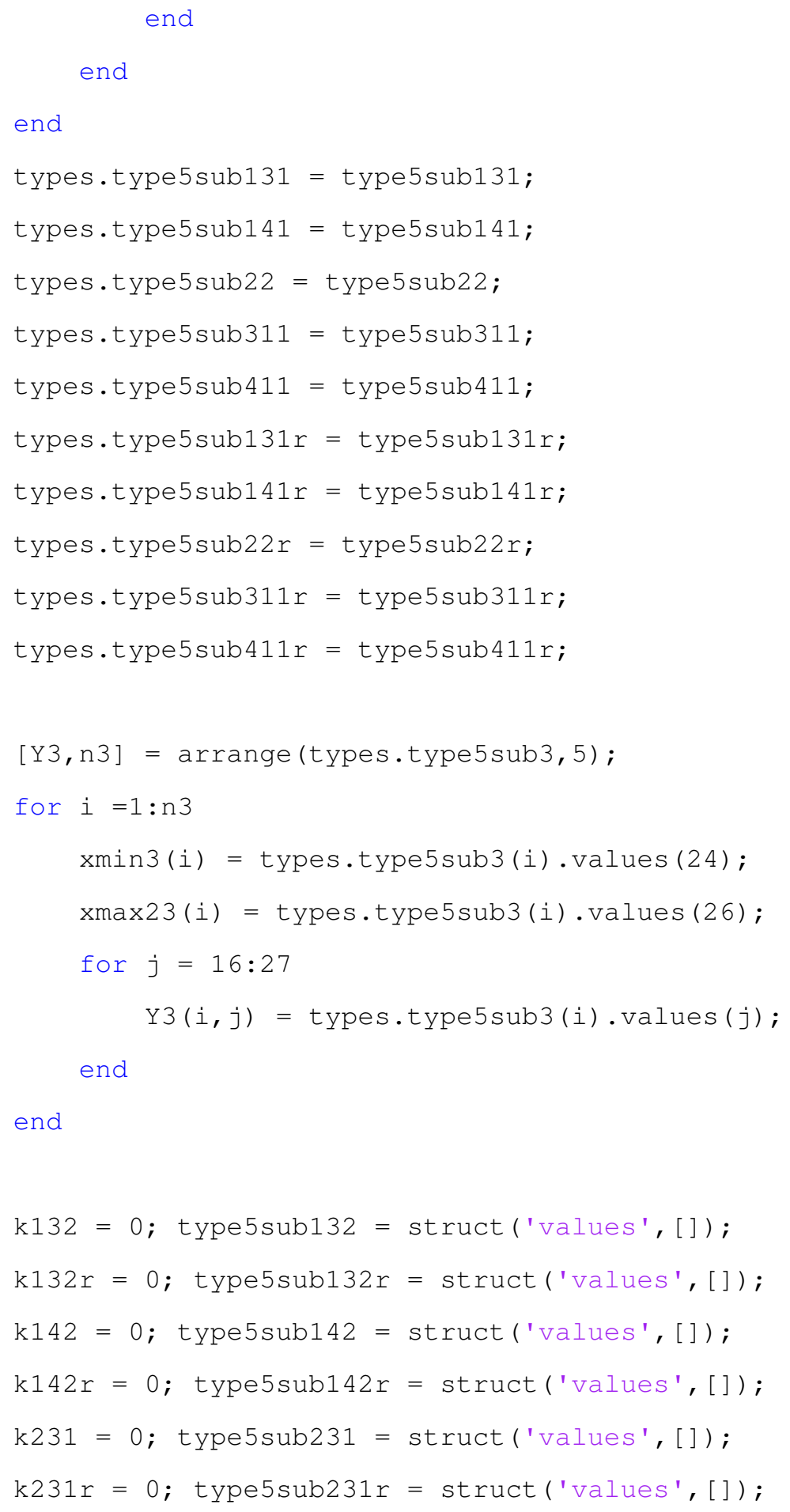




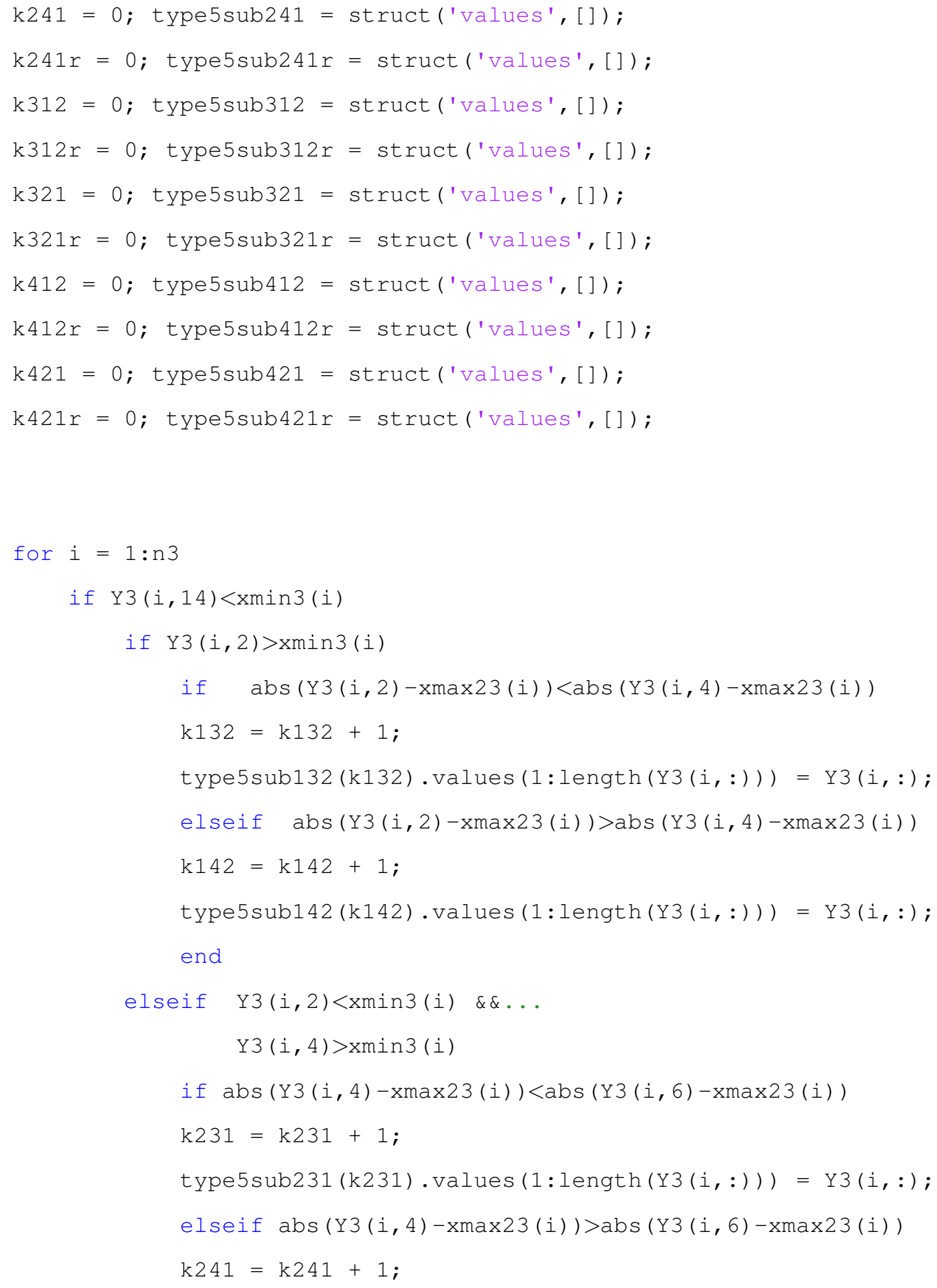




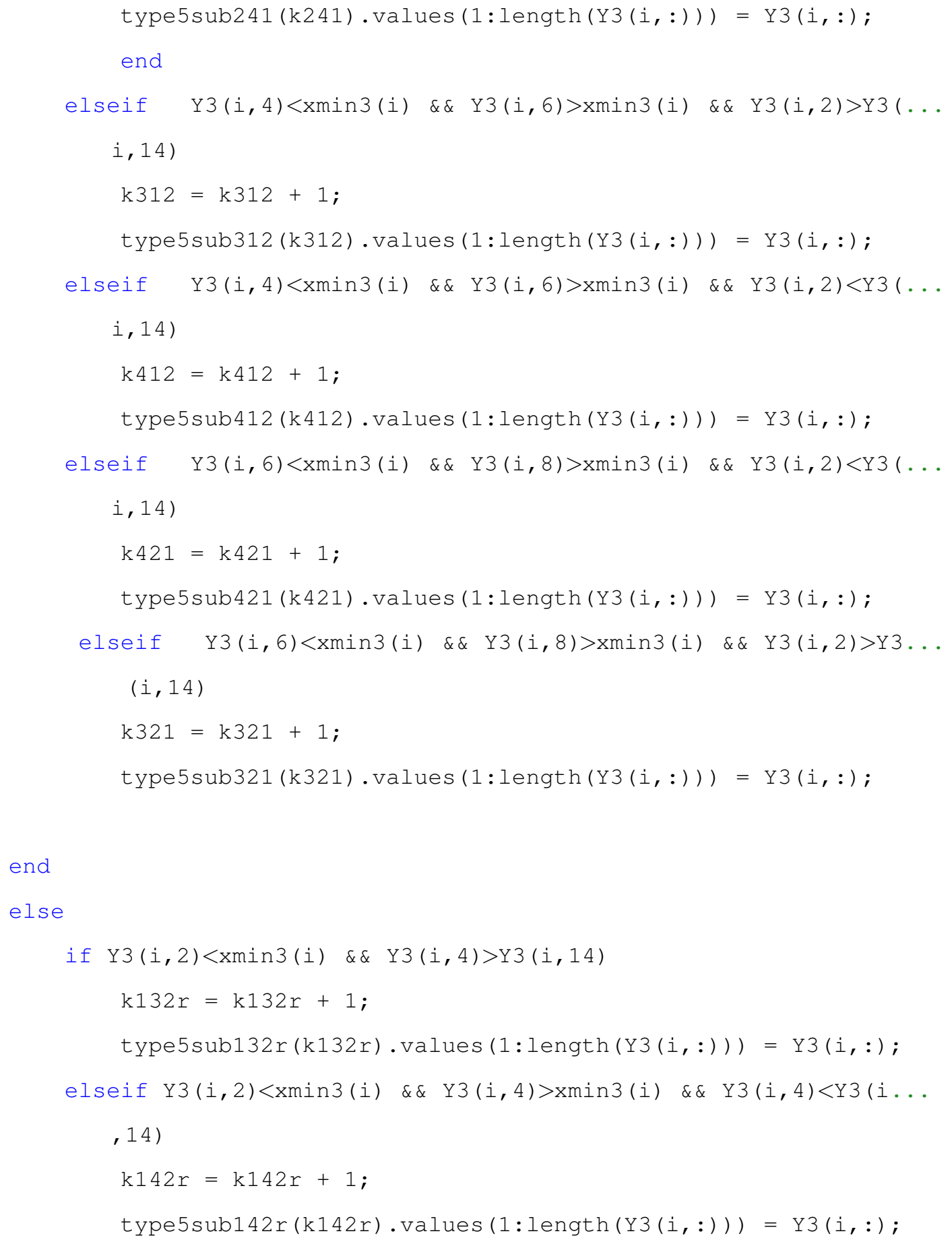




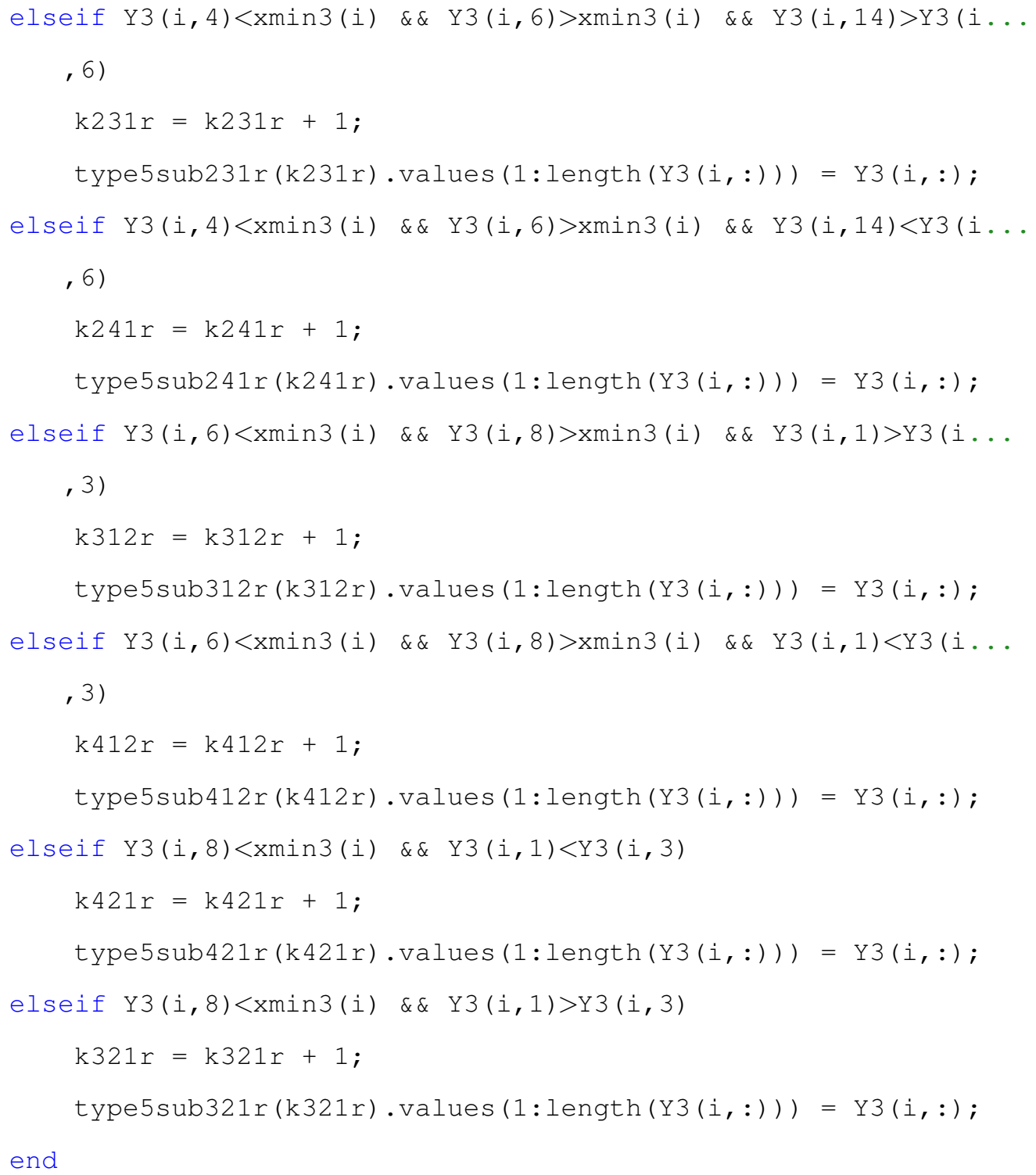




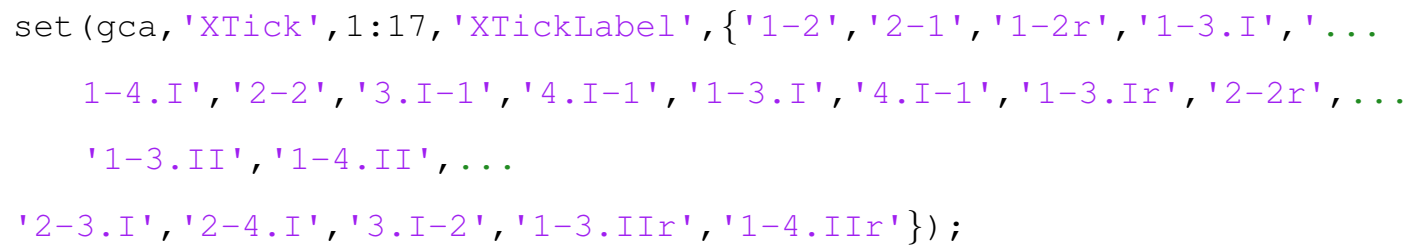

end 


\section{Appendix E}

\section{Synthetic Data Generation Code}

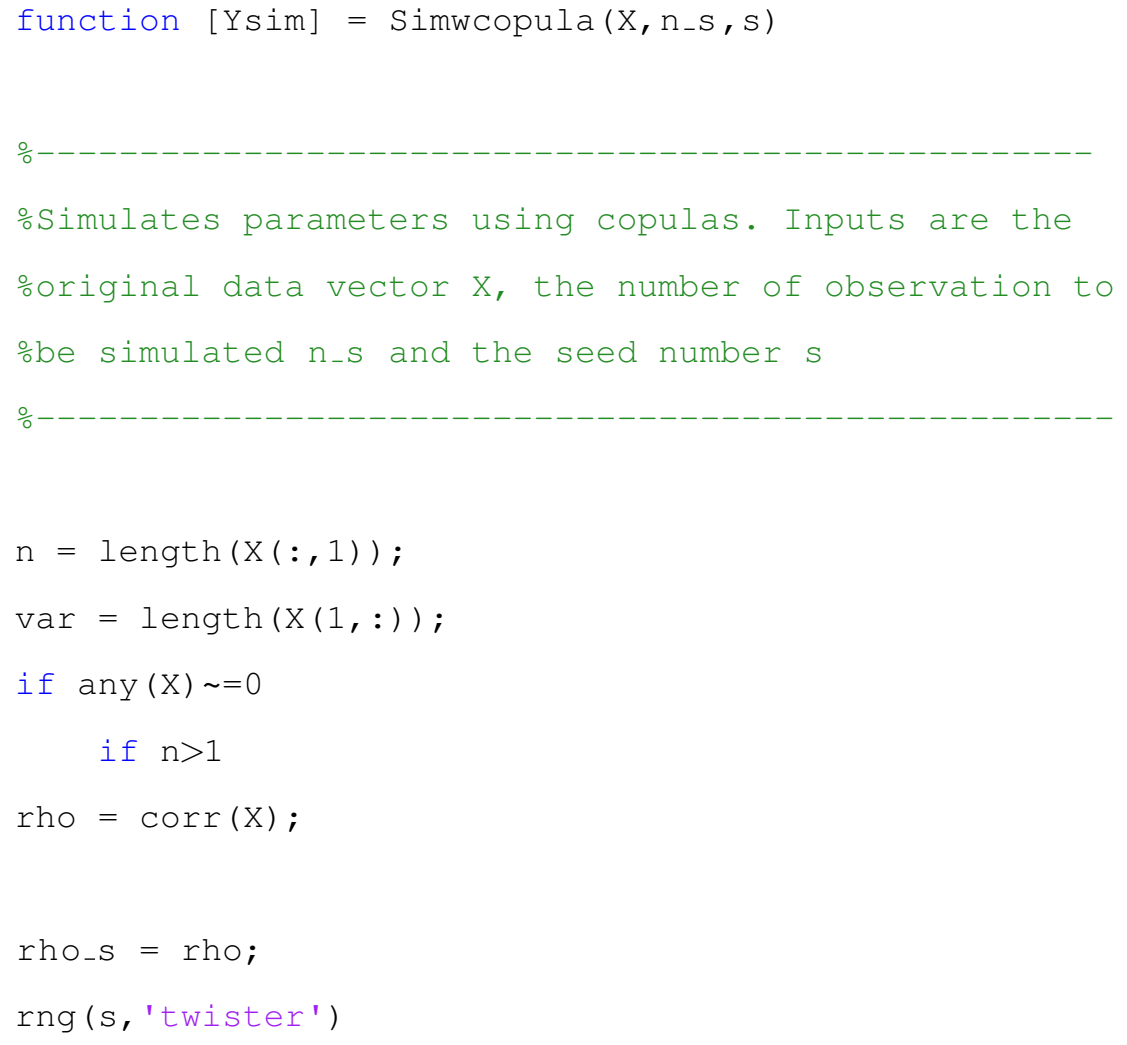



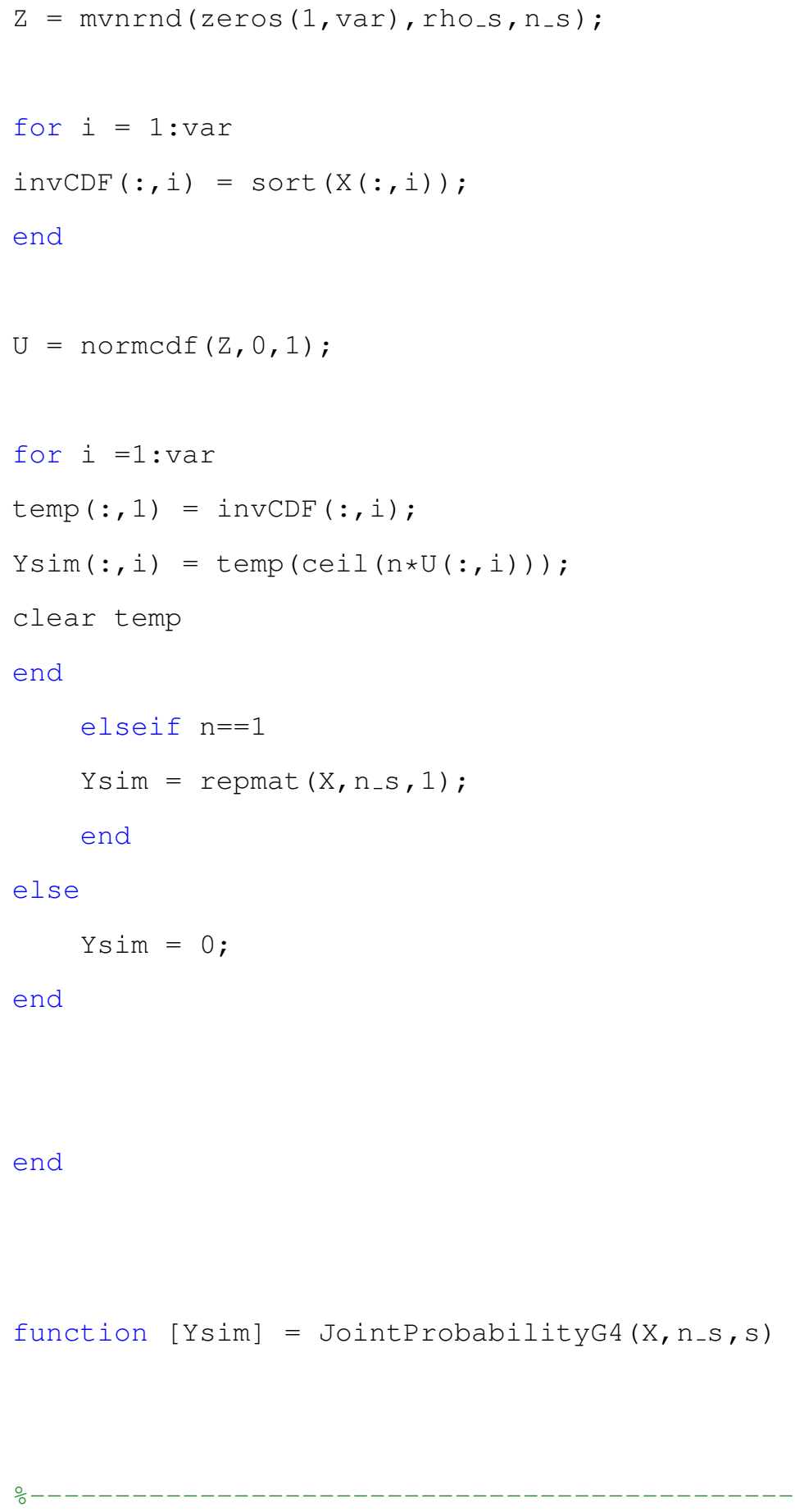

oSimulates data using the histogram method. 


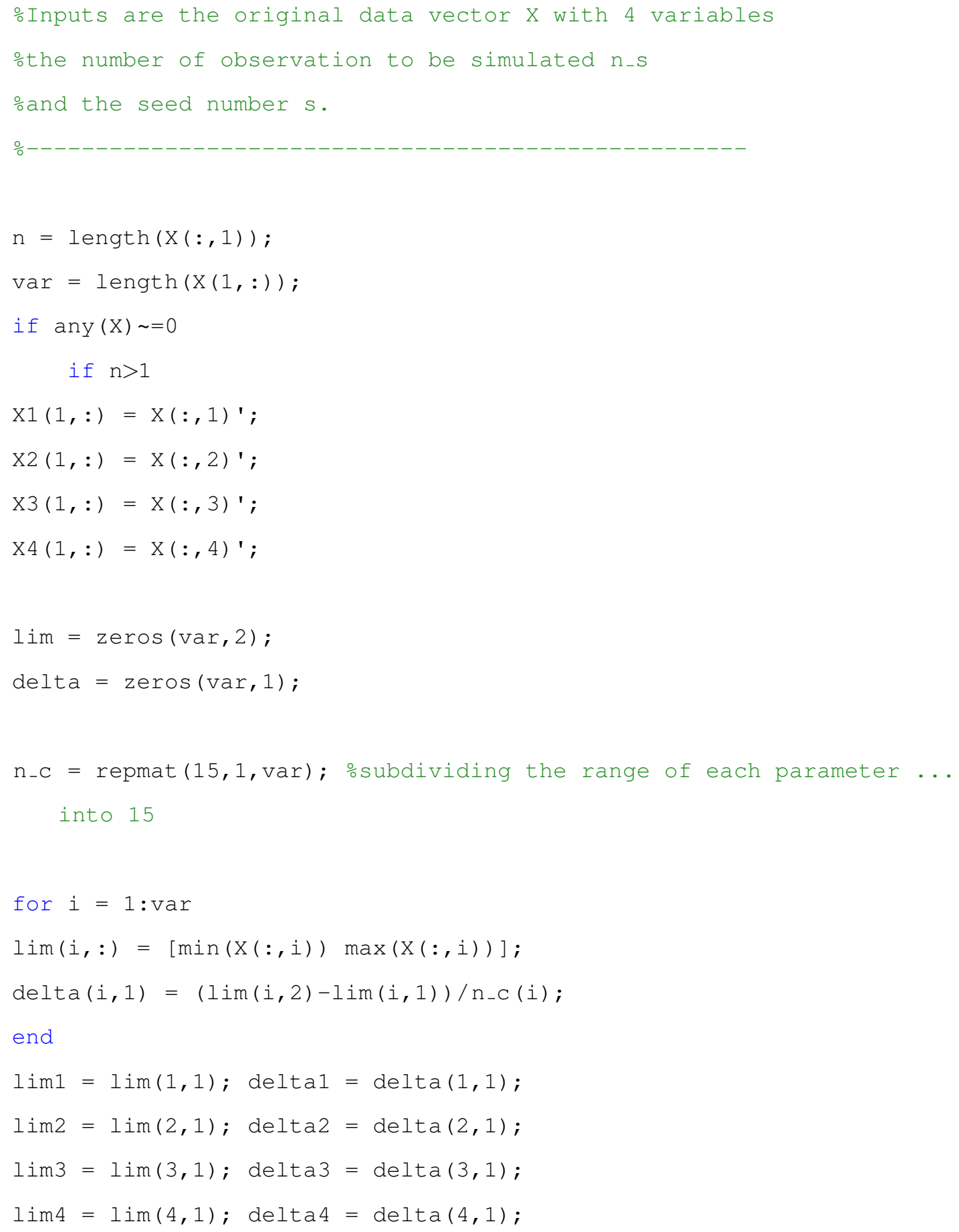




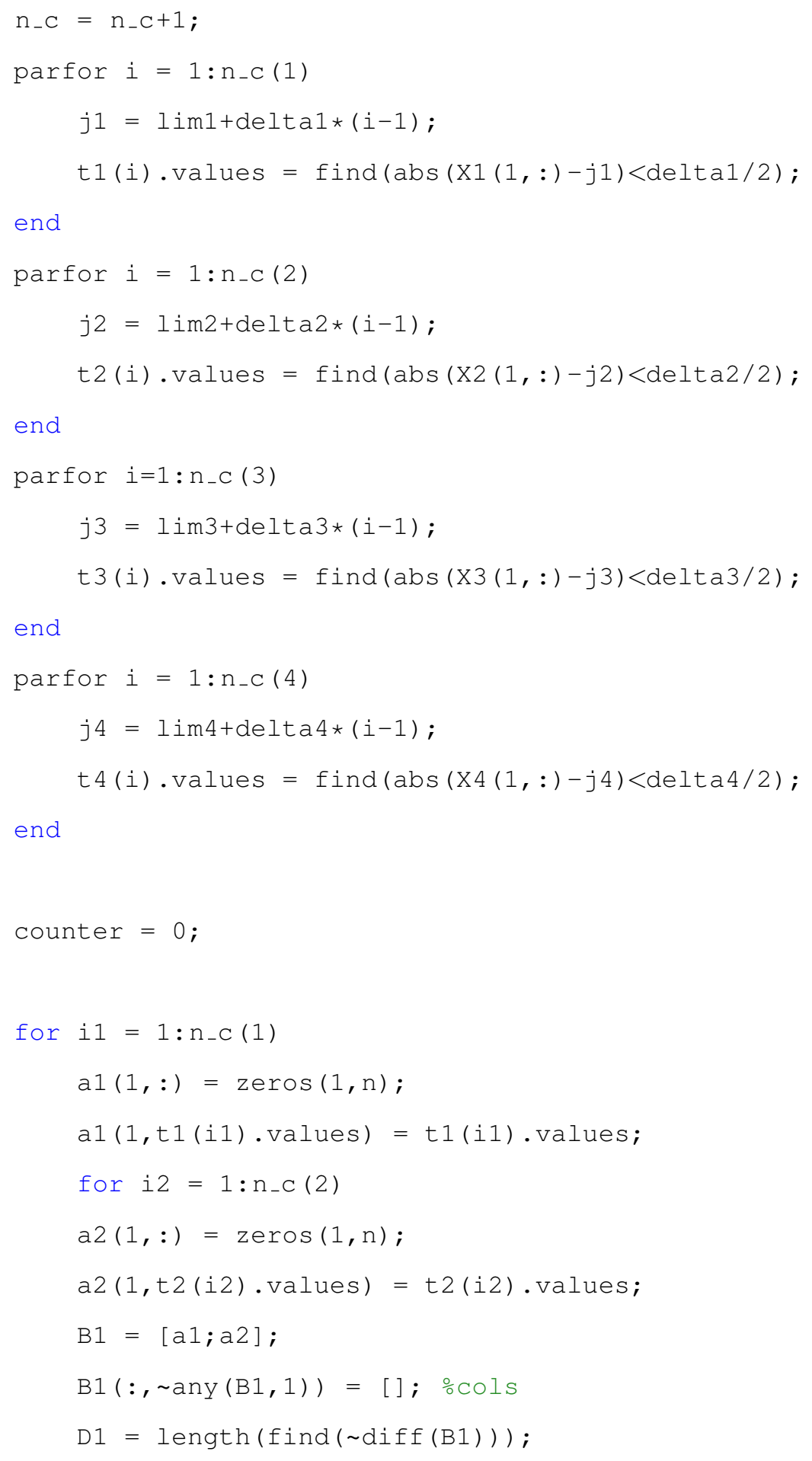




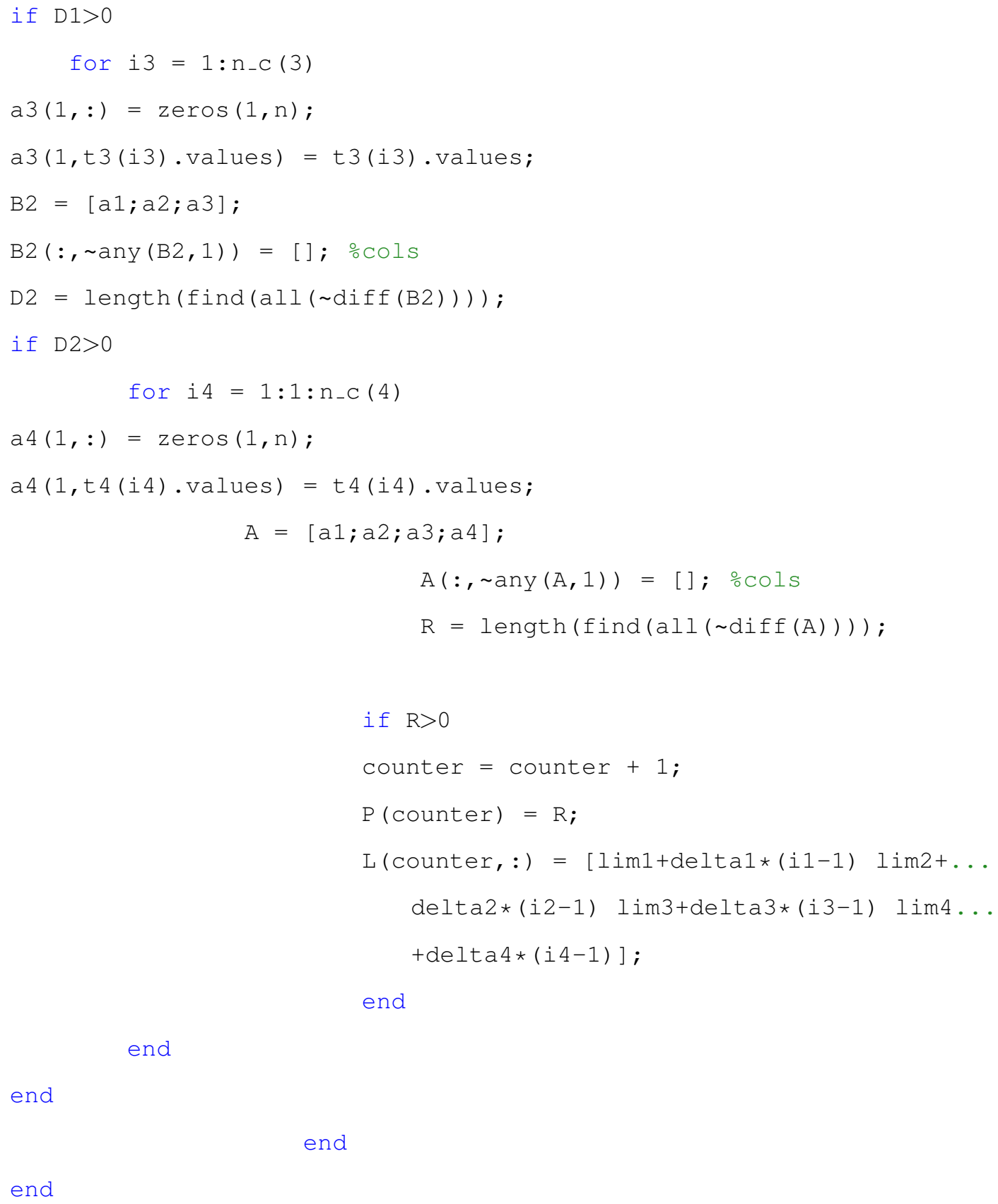




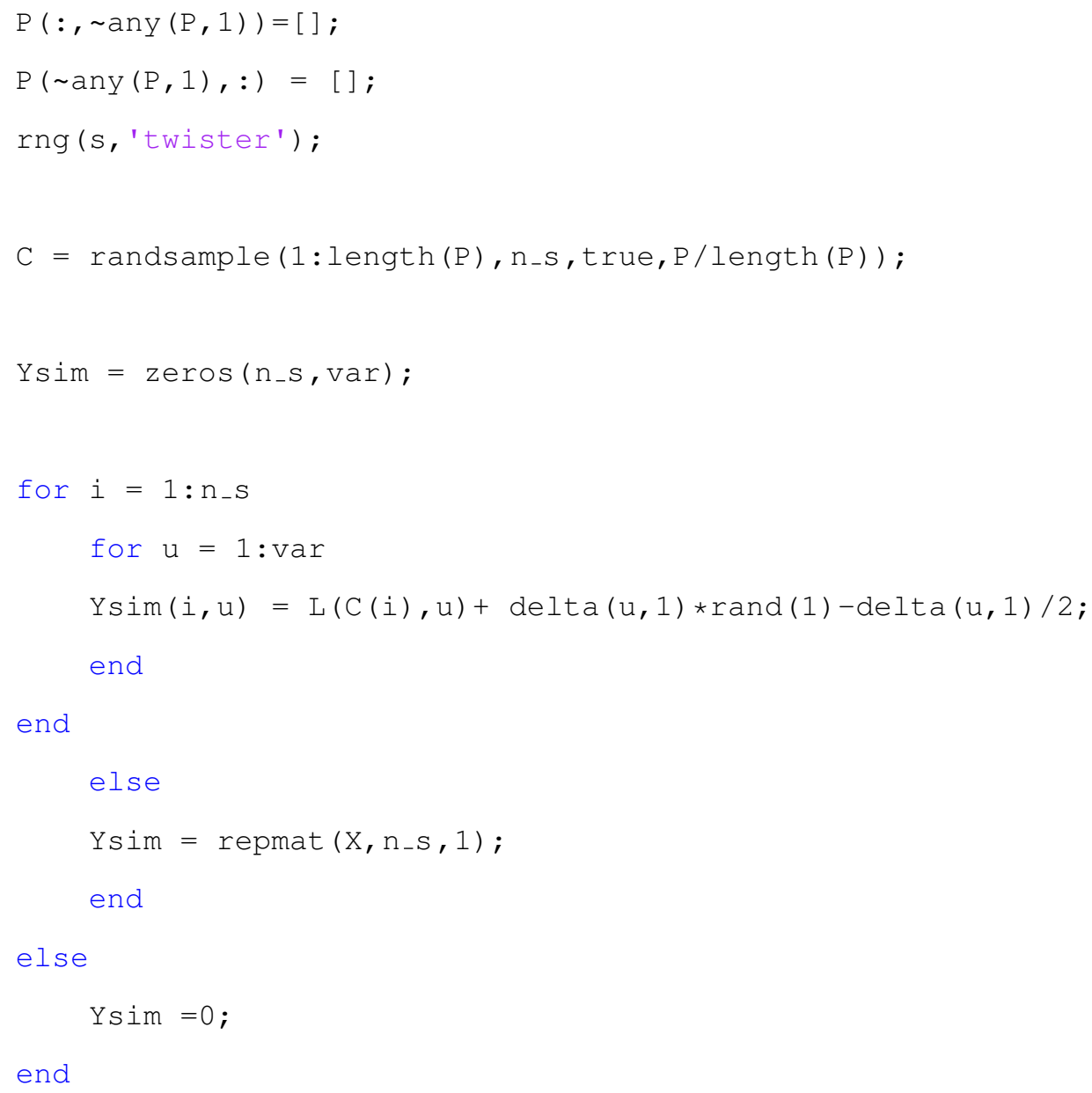




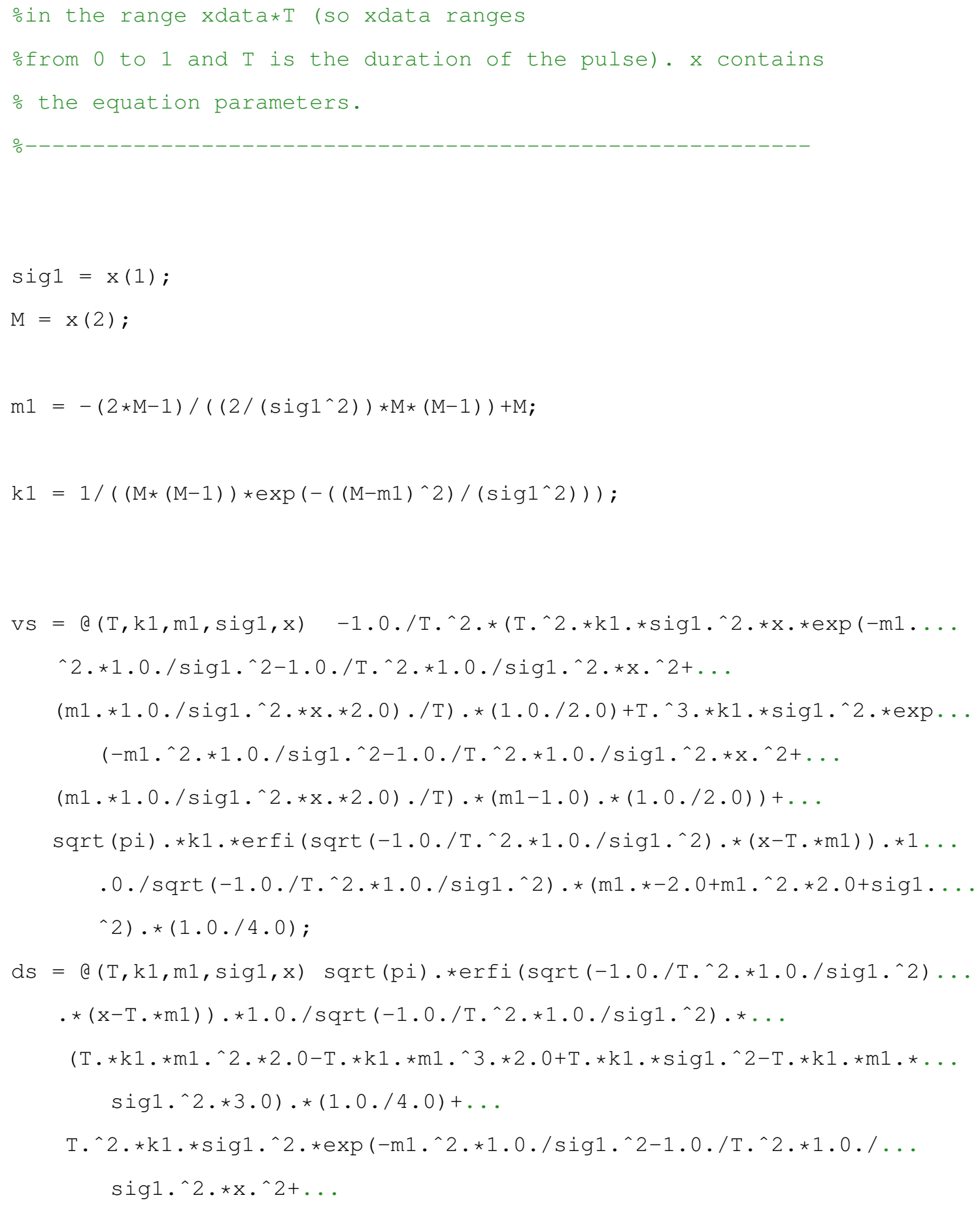




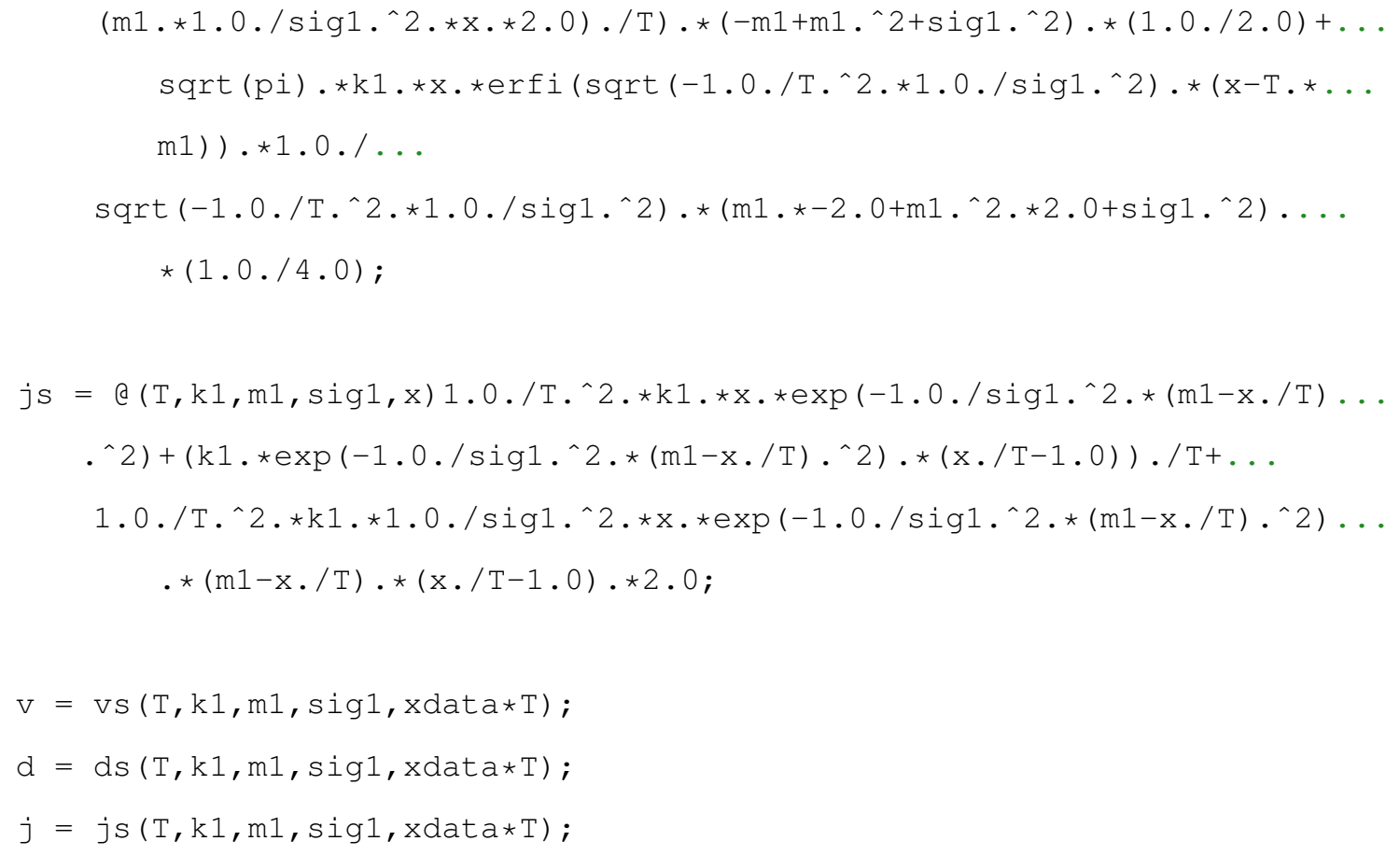

end

function [generatedpeak, gtypes, az, timevec, pres, vz, dz] = ..

generatedatanewlast2 (types, N, s)

oGenerates time history with $\mathrm{N}-1$ pulses using copulas

obased on the statistics of actual data which is in

othe structure types. The outputs are the structure

ogeneratedpeak which is similar to the structure peak

\%(e.g. generatedpeak.az(n) $\cdot \operatorname{values}(:)$ =acceleration

ovalues of nth pulse), the time vector timevec, the 


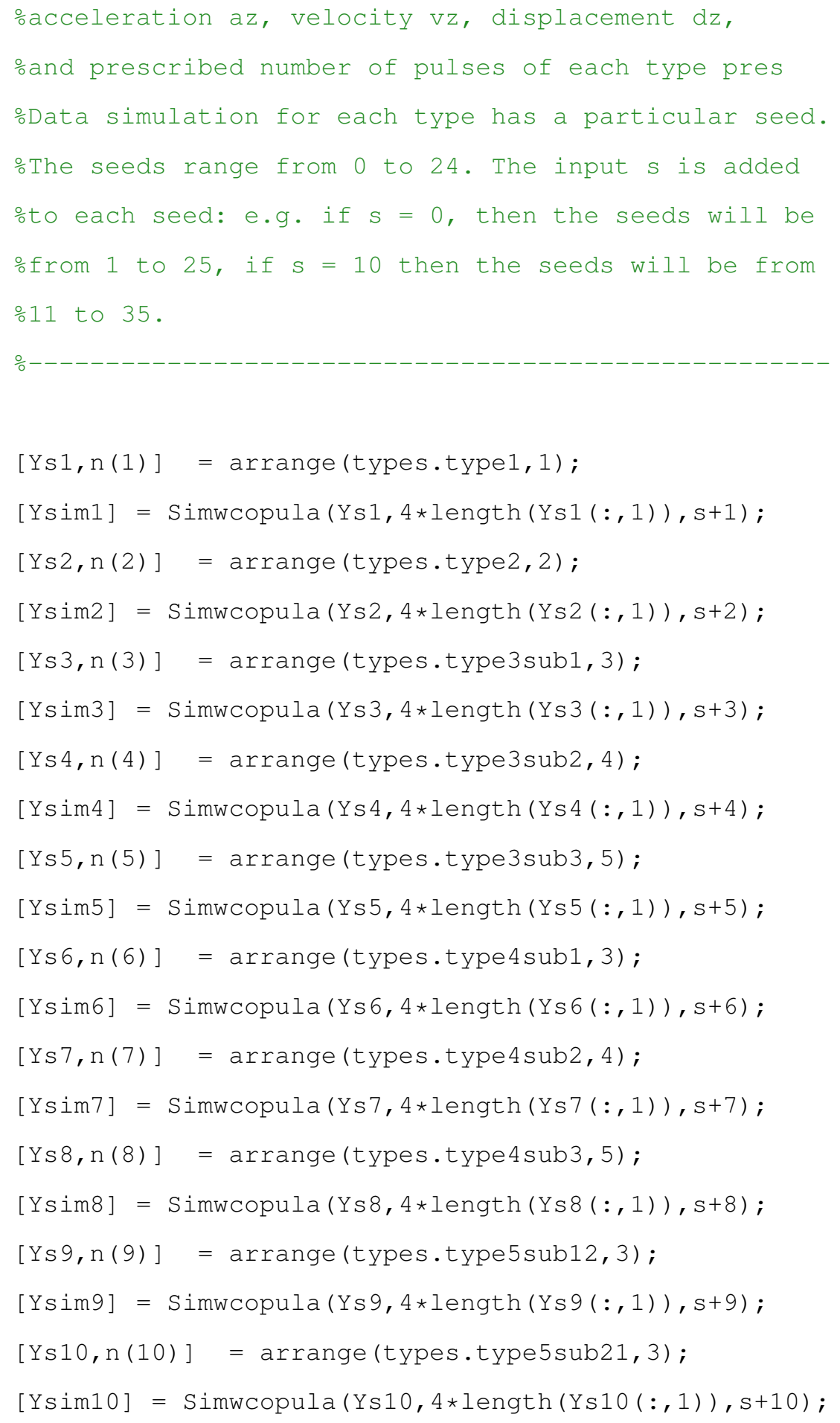




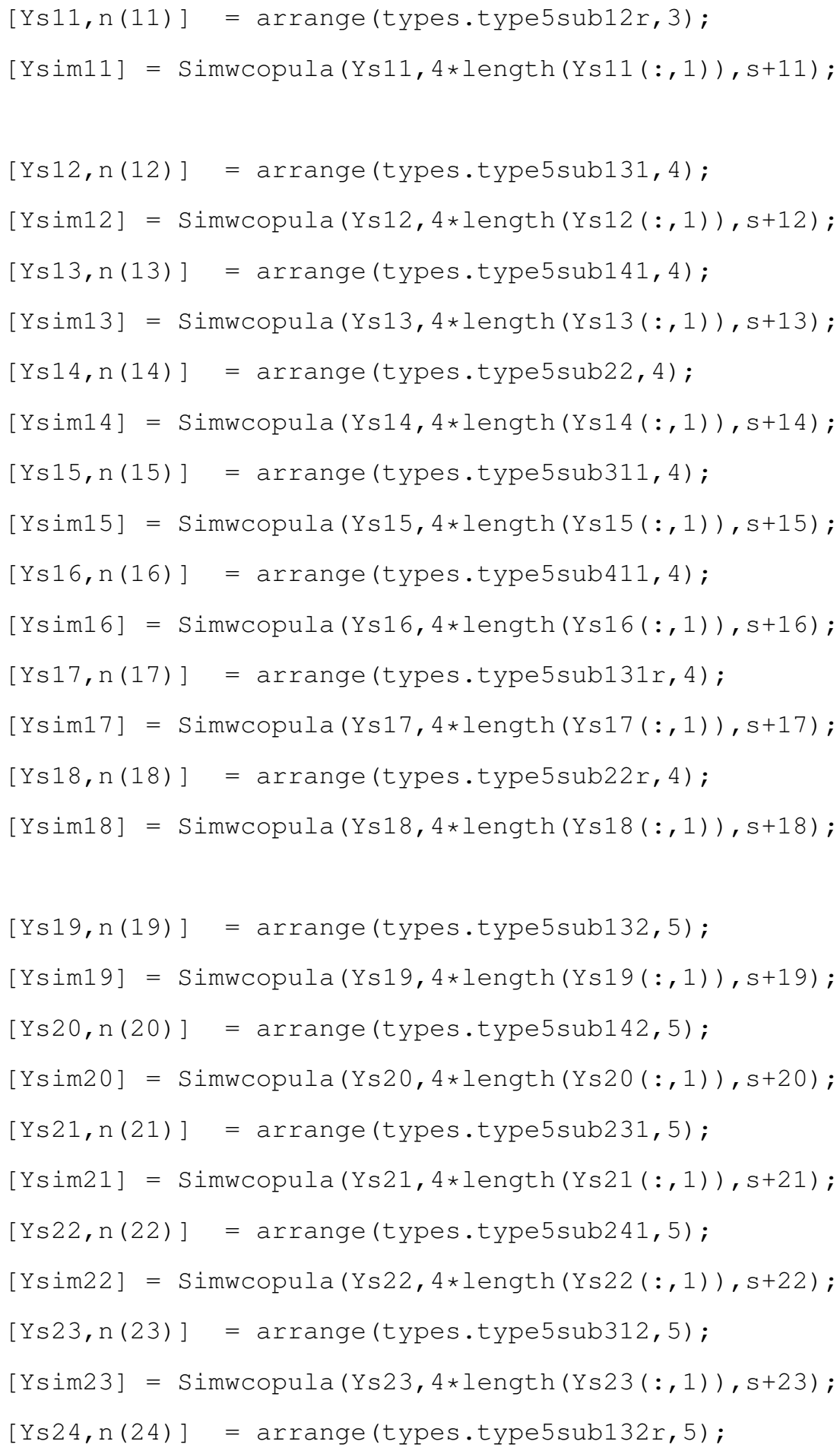




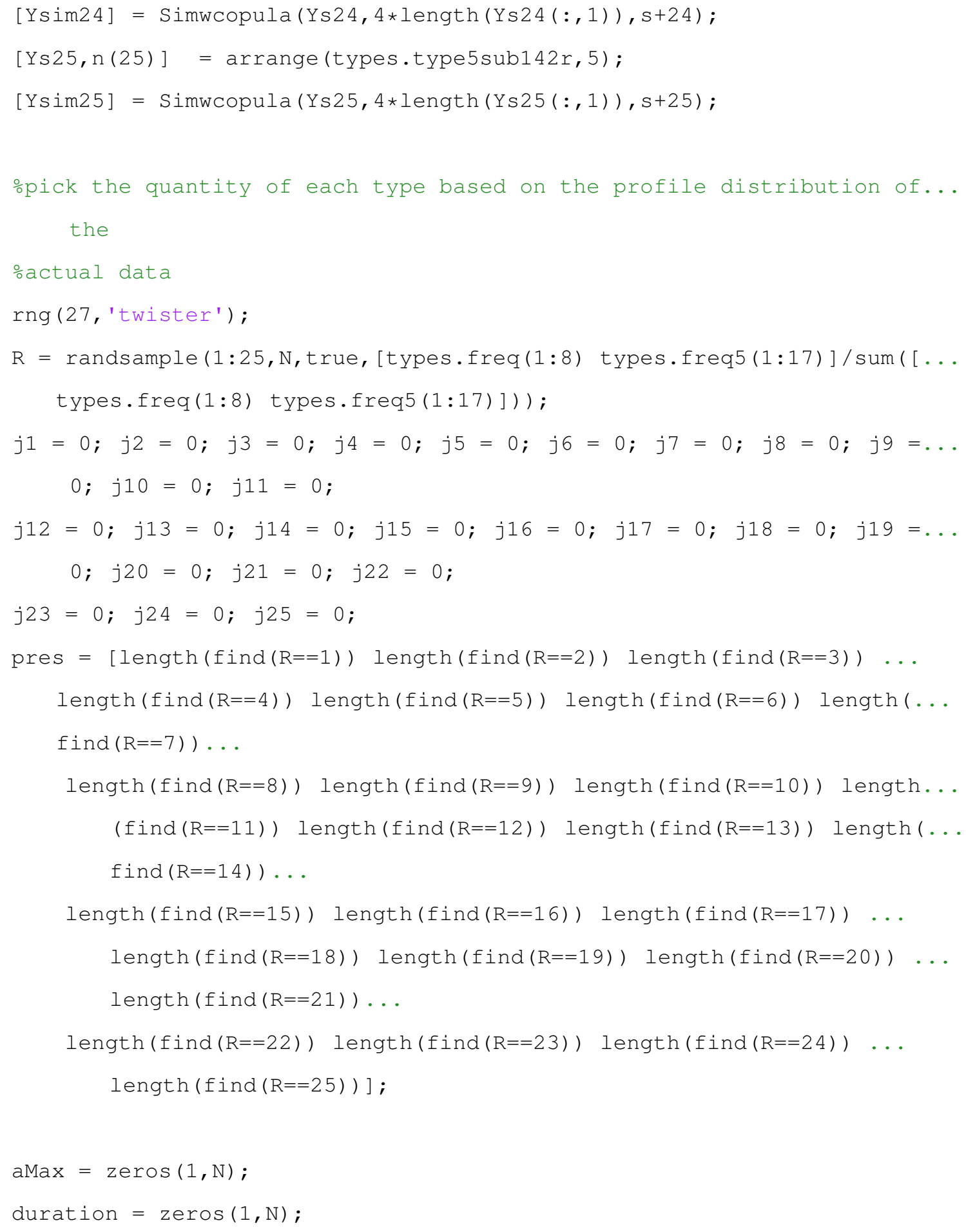




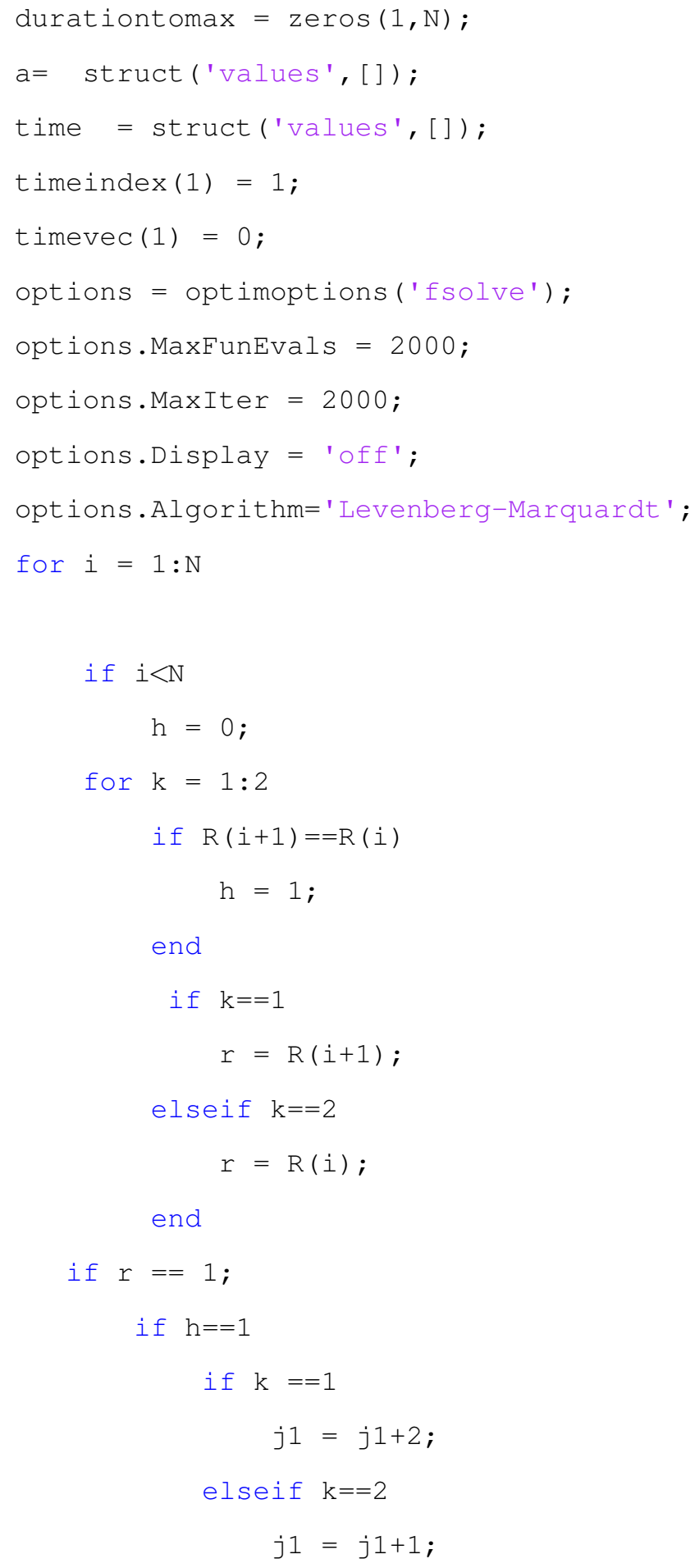




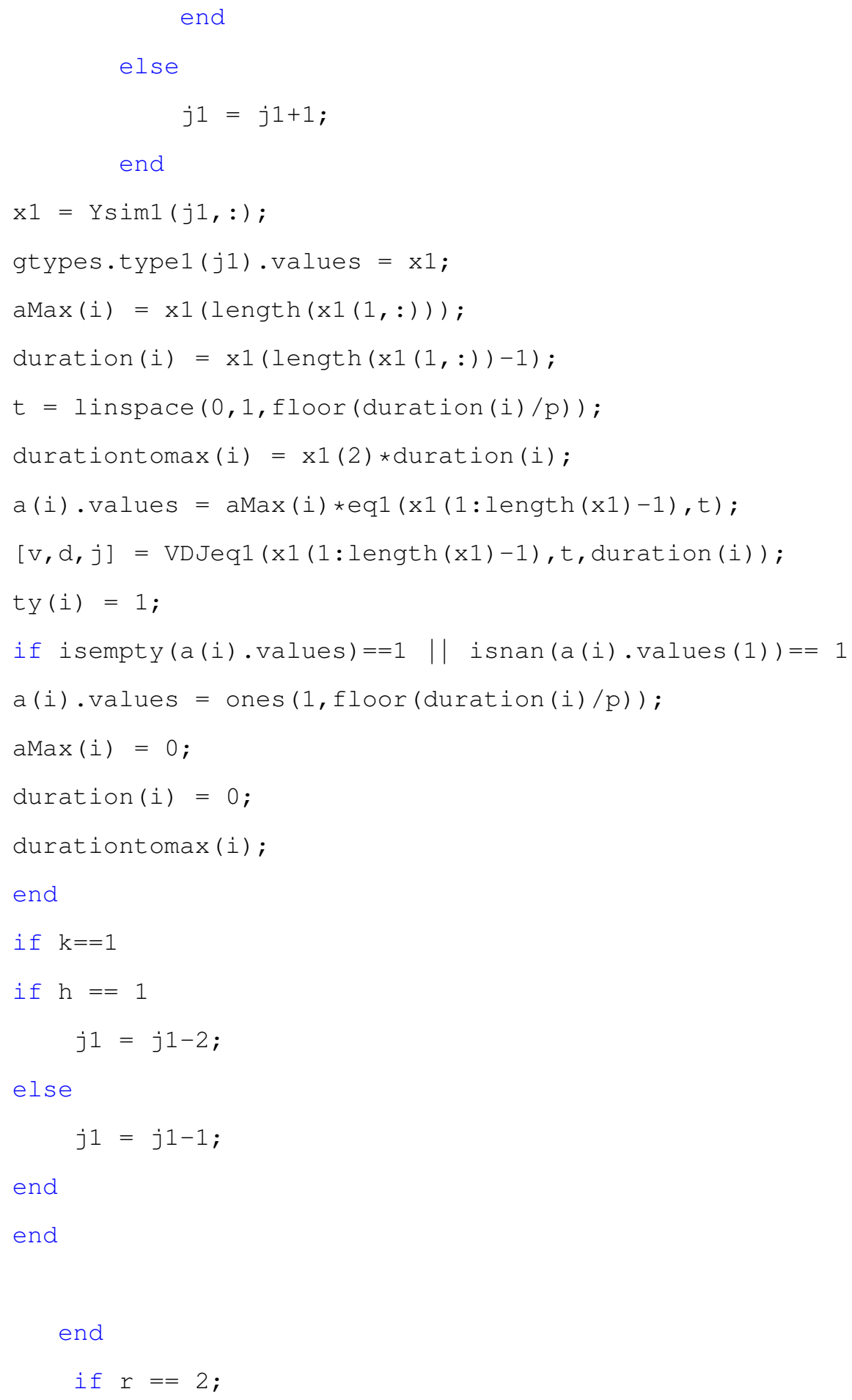




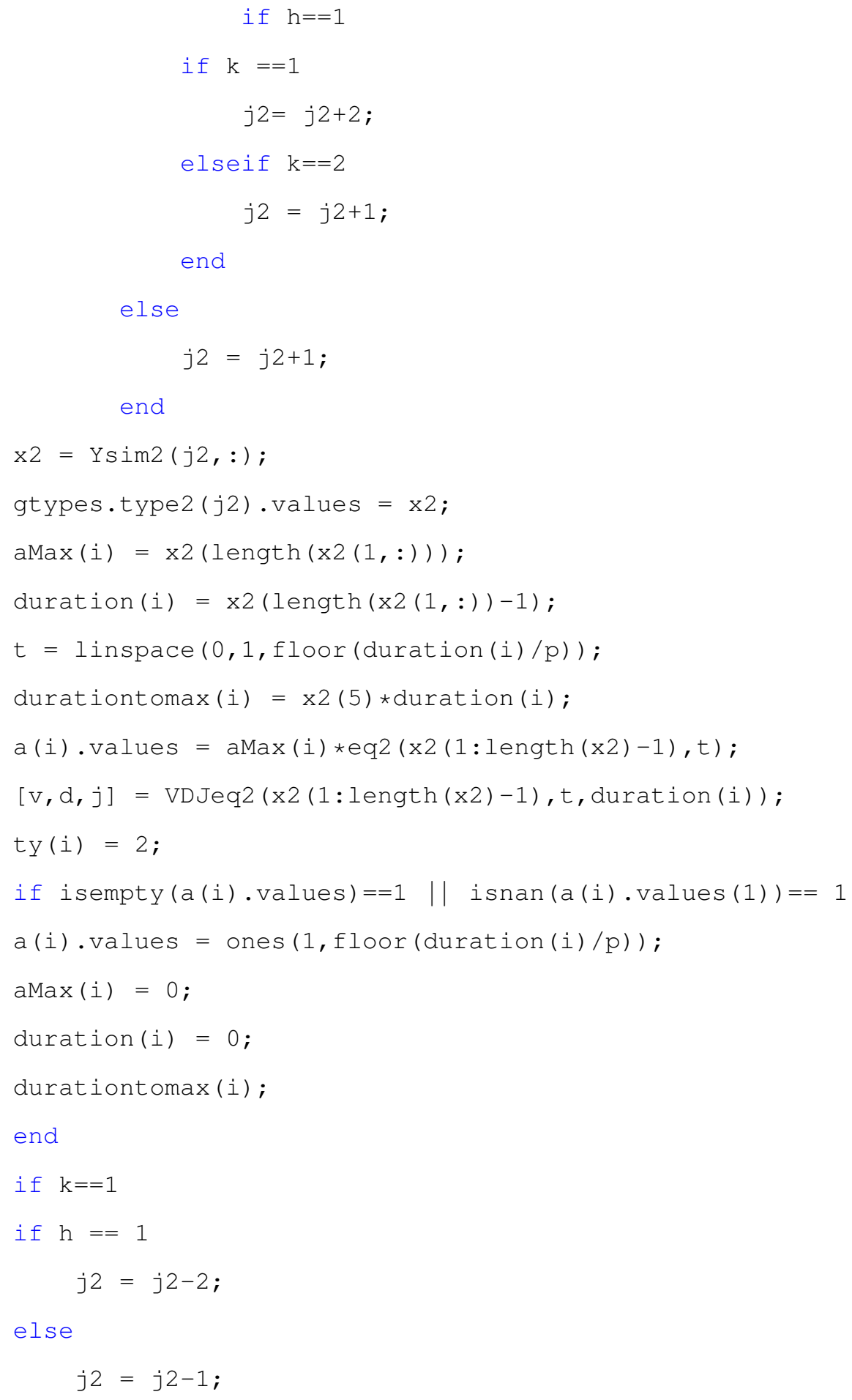




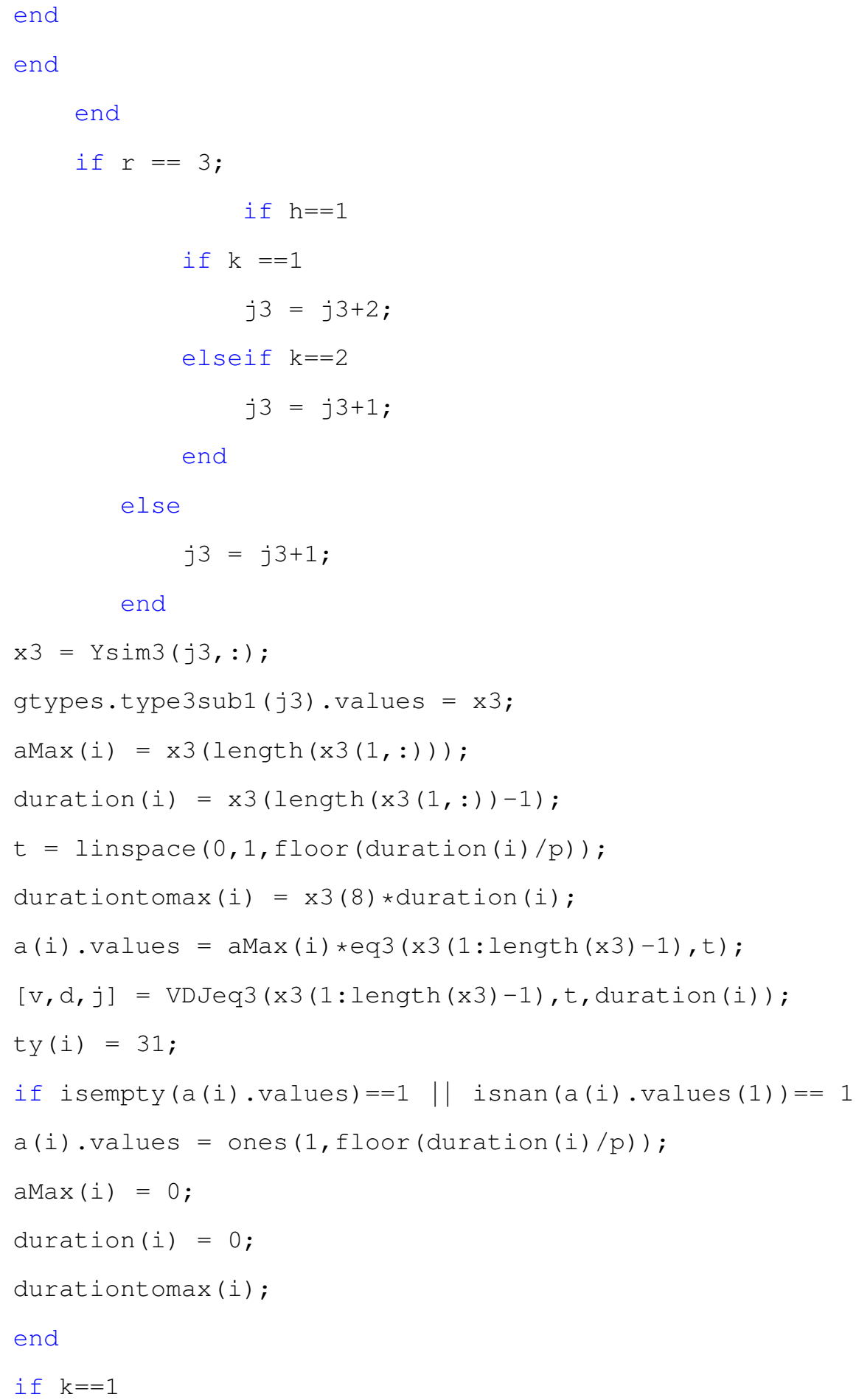




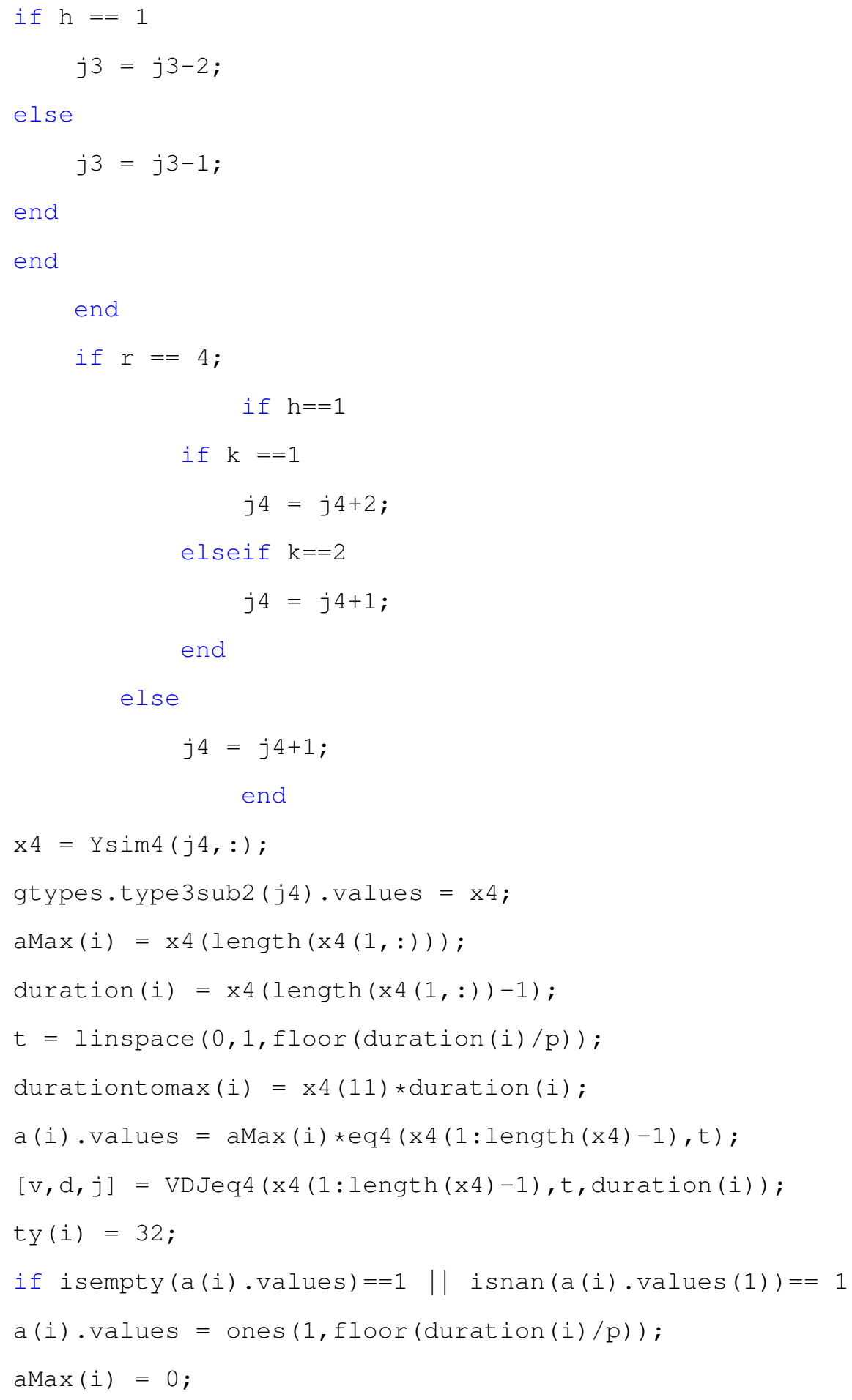




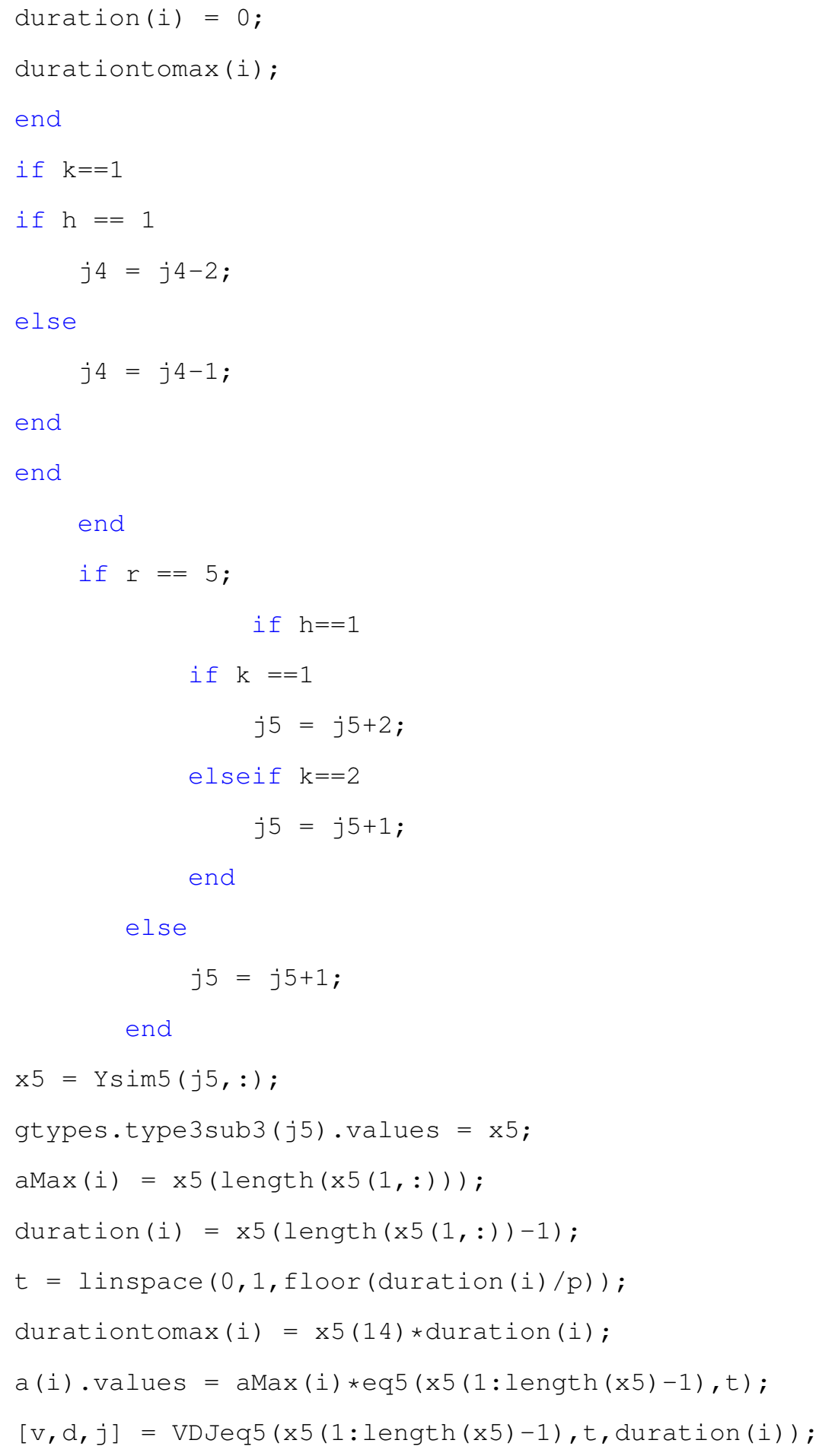




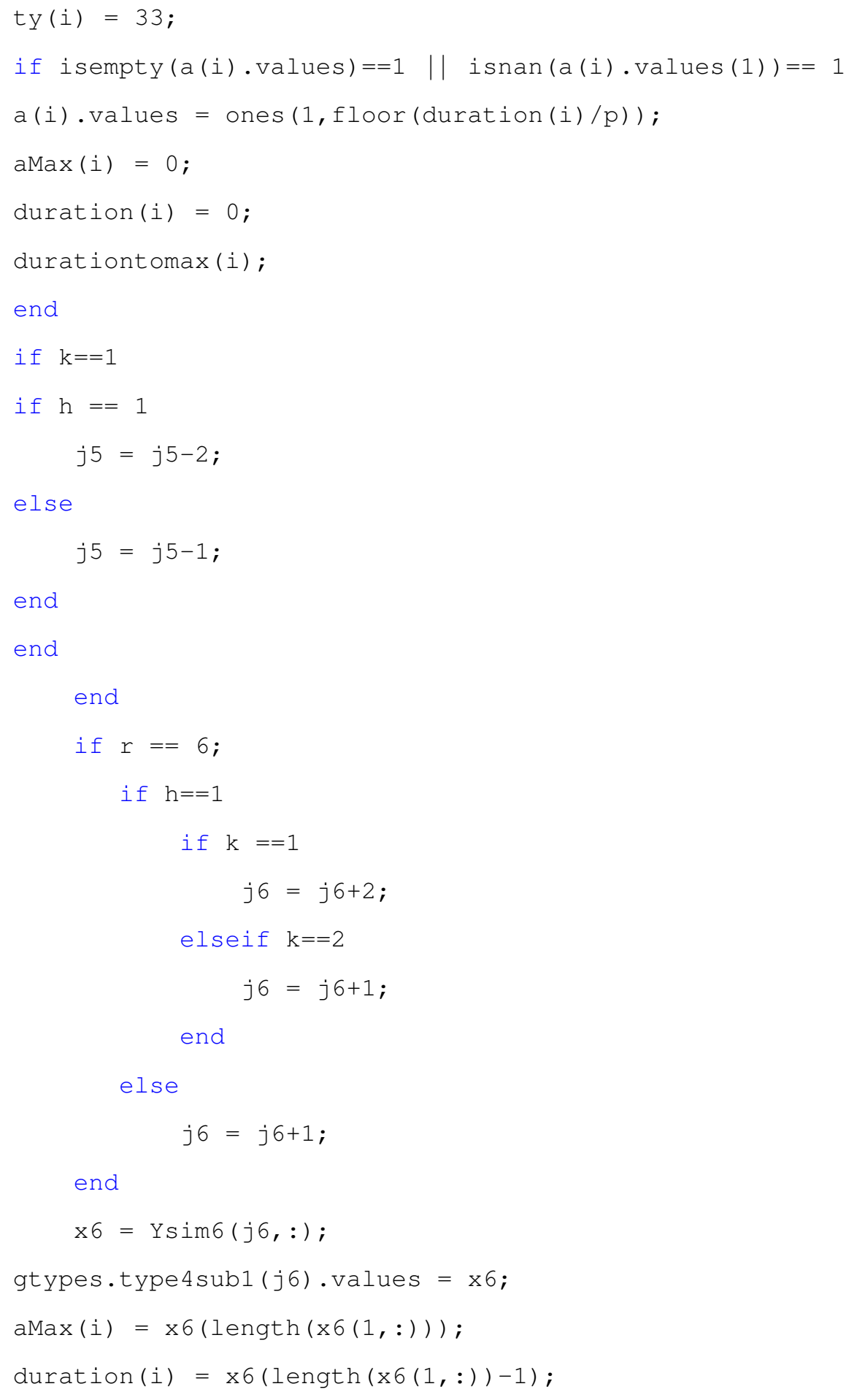




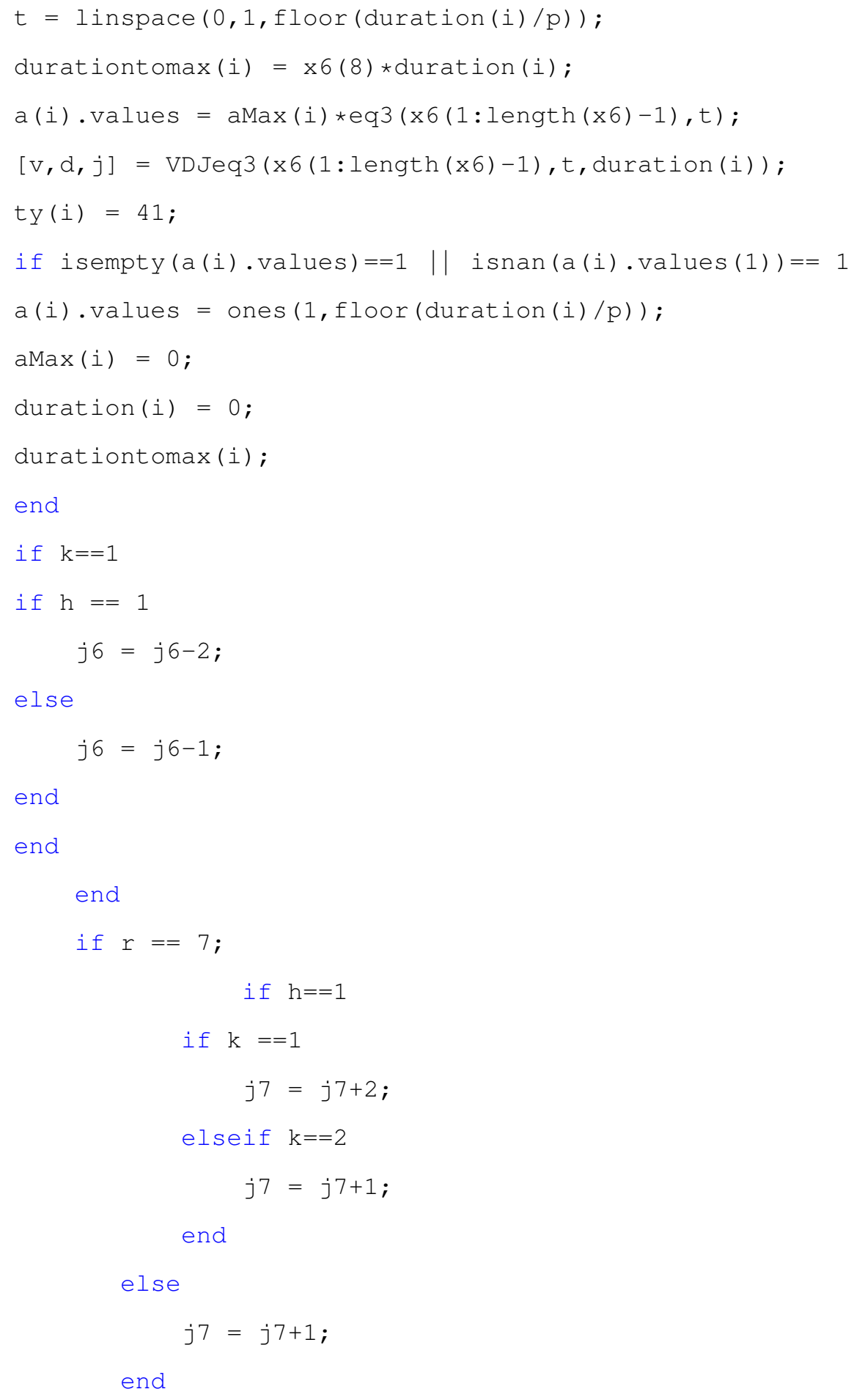




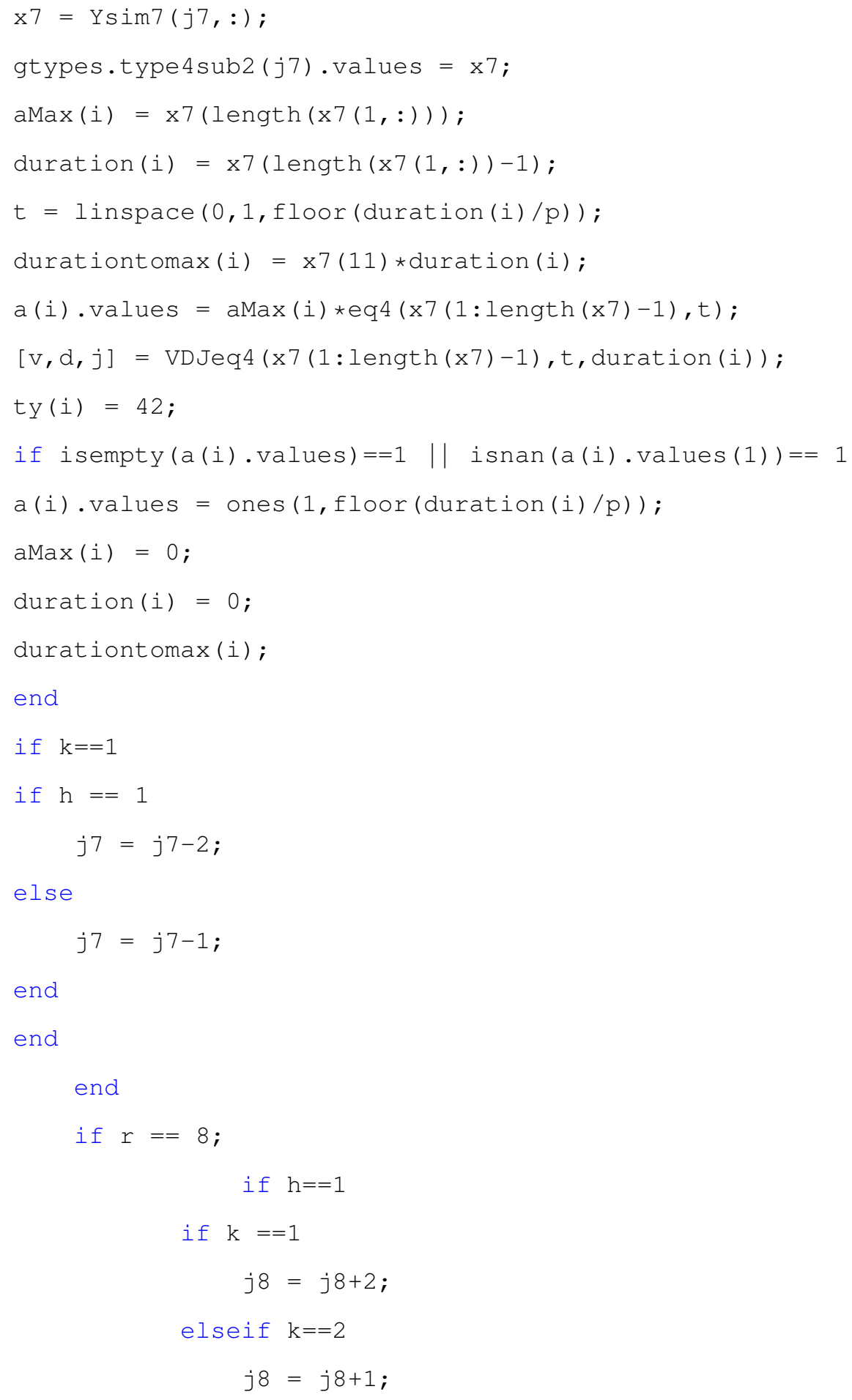




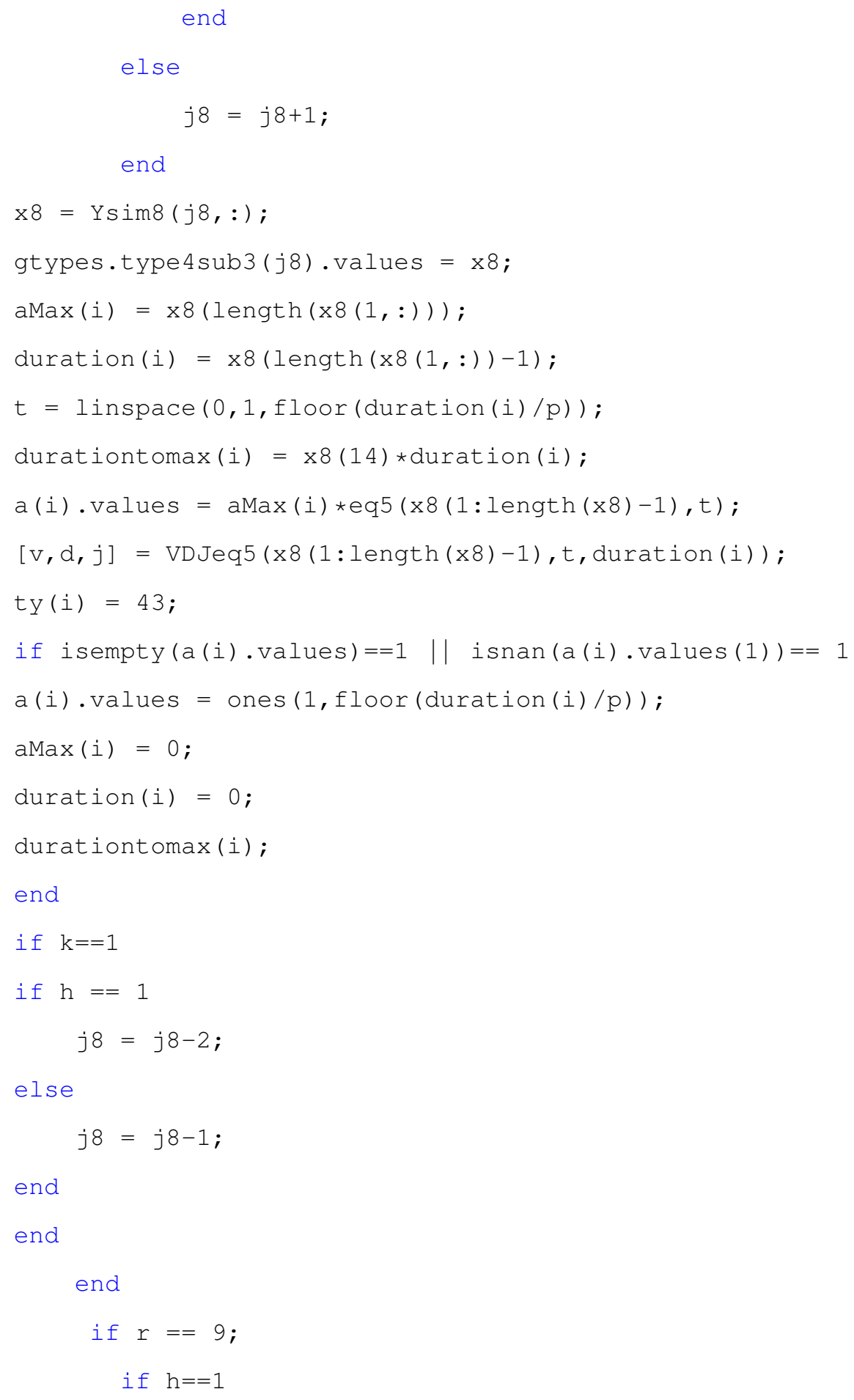




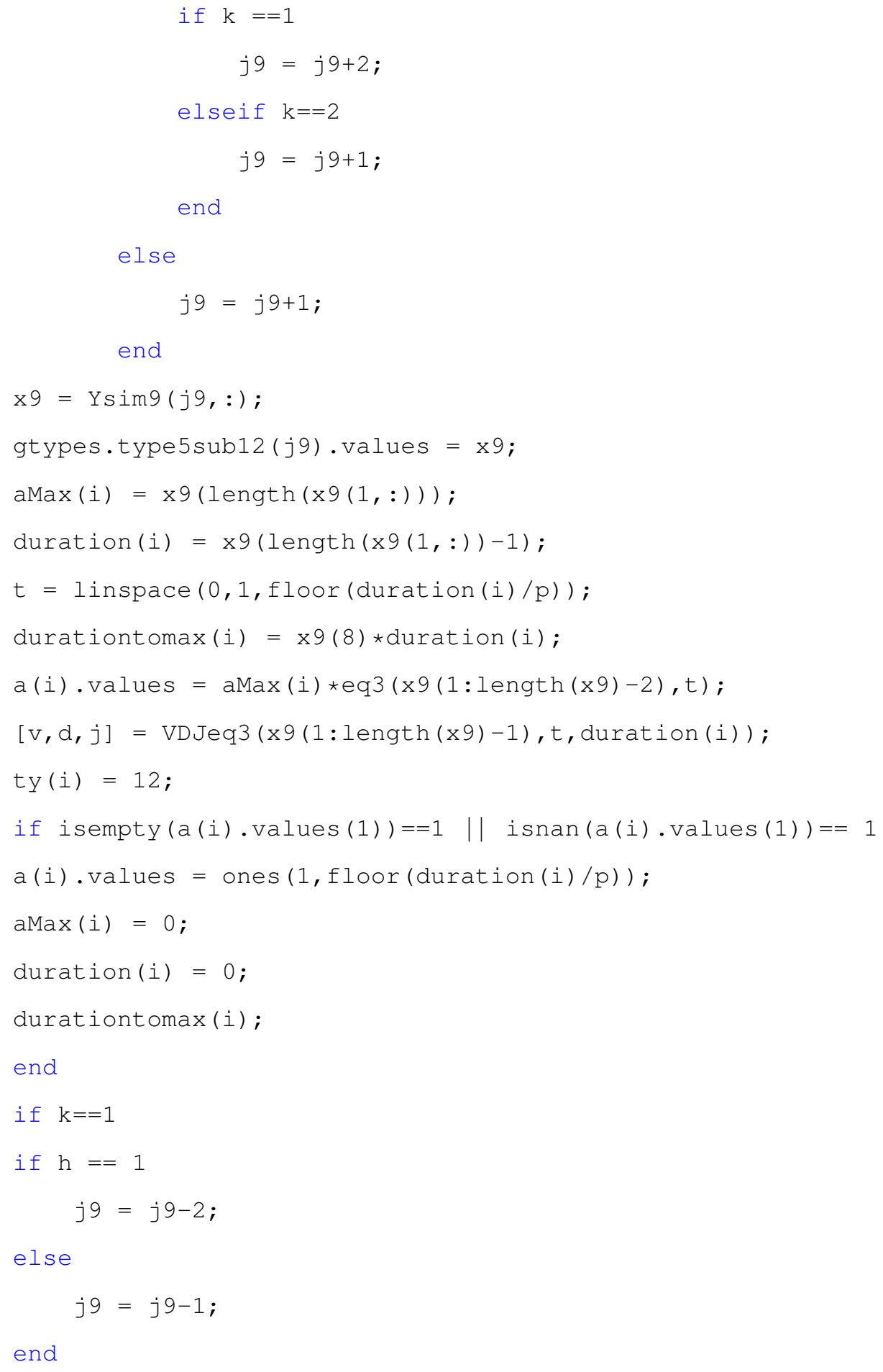




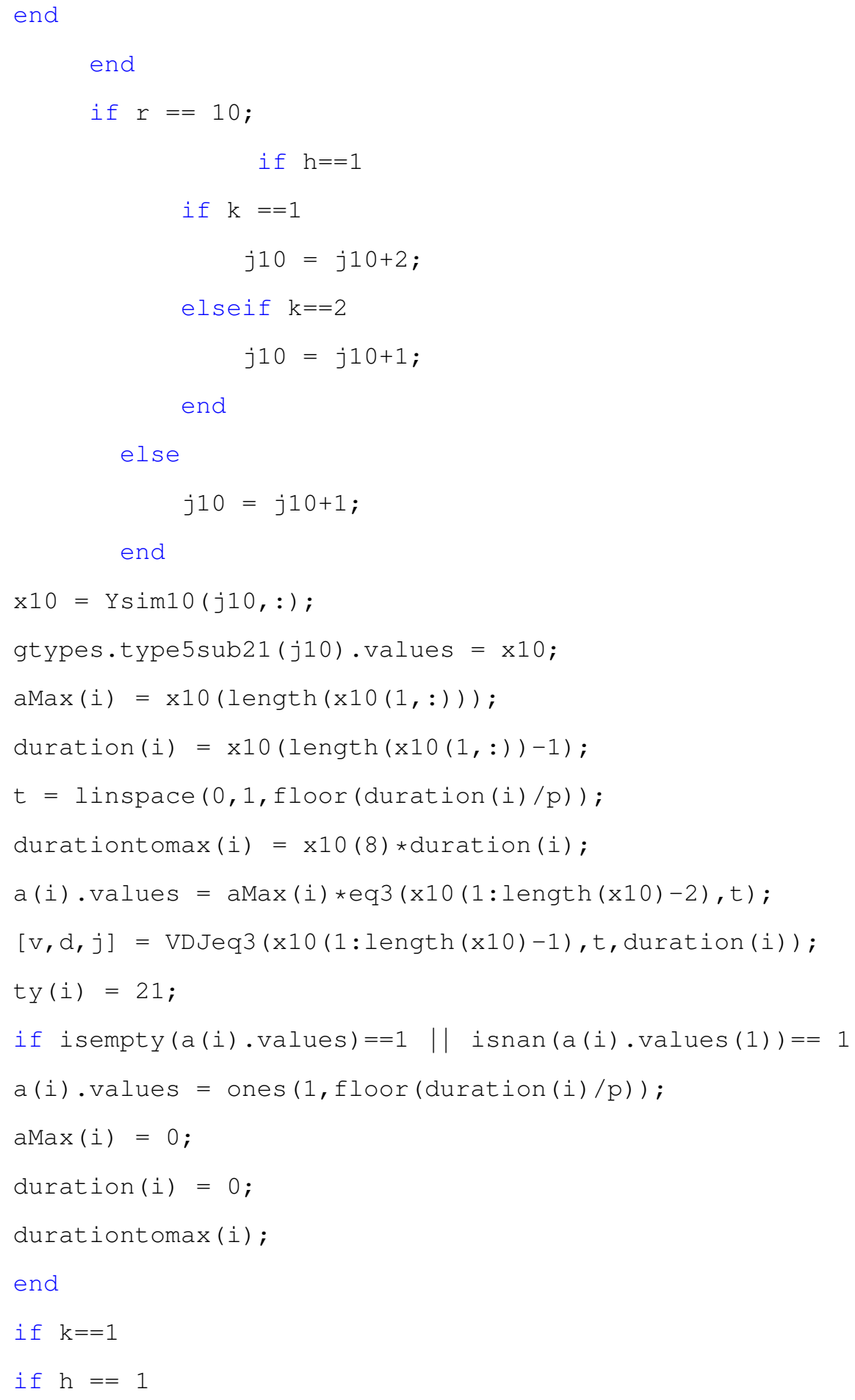




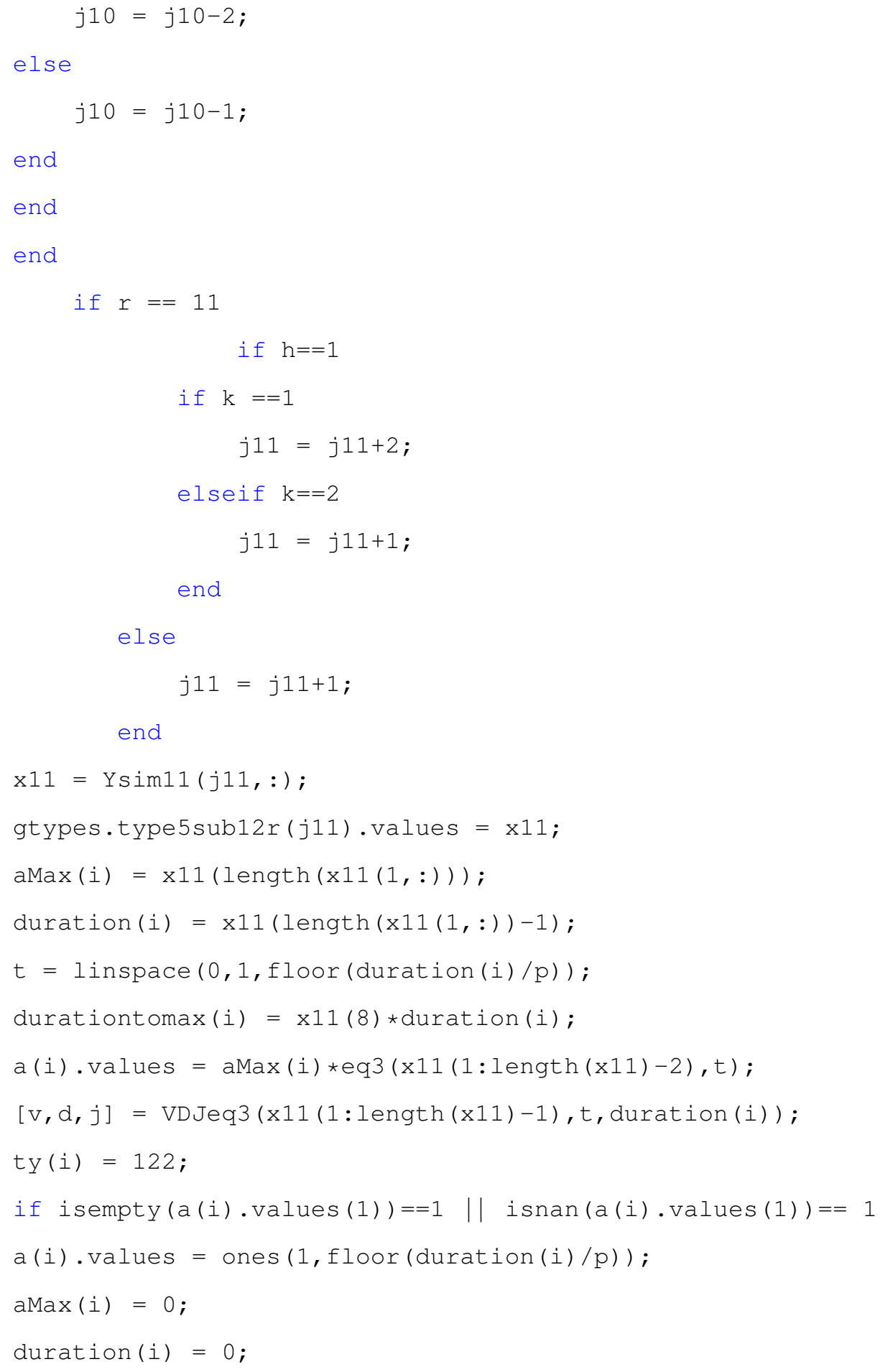




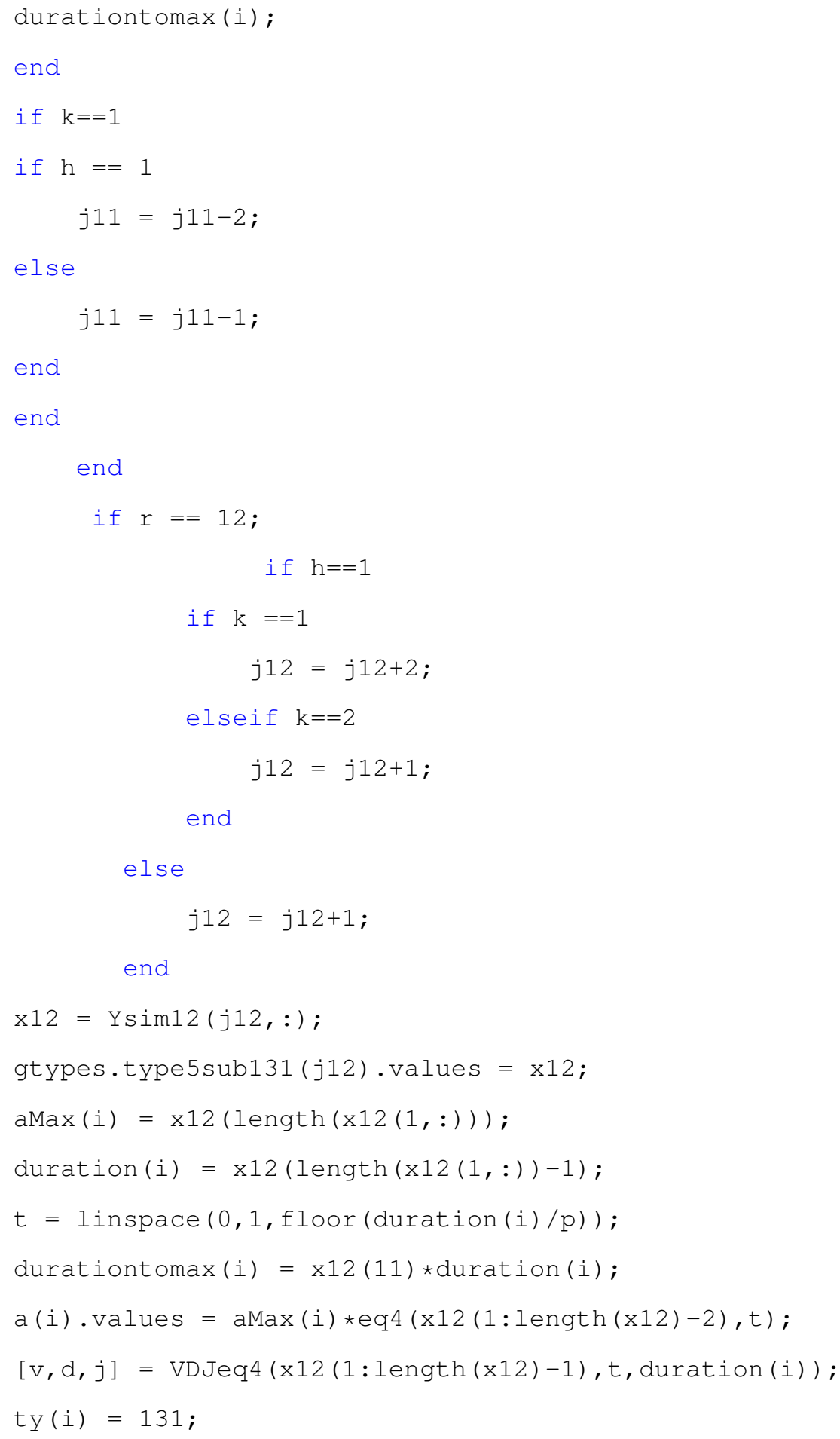




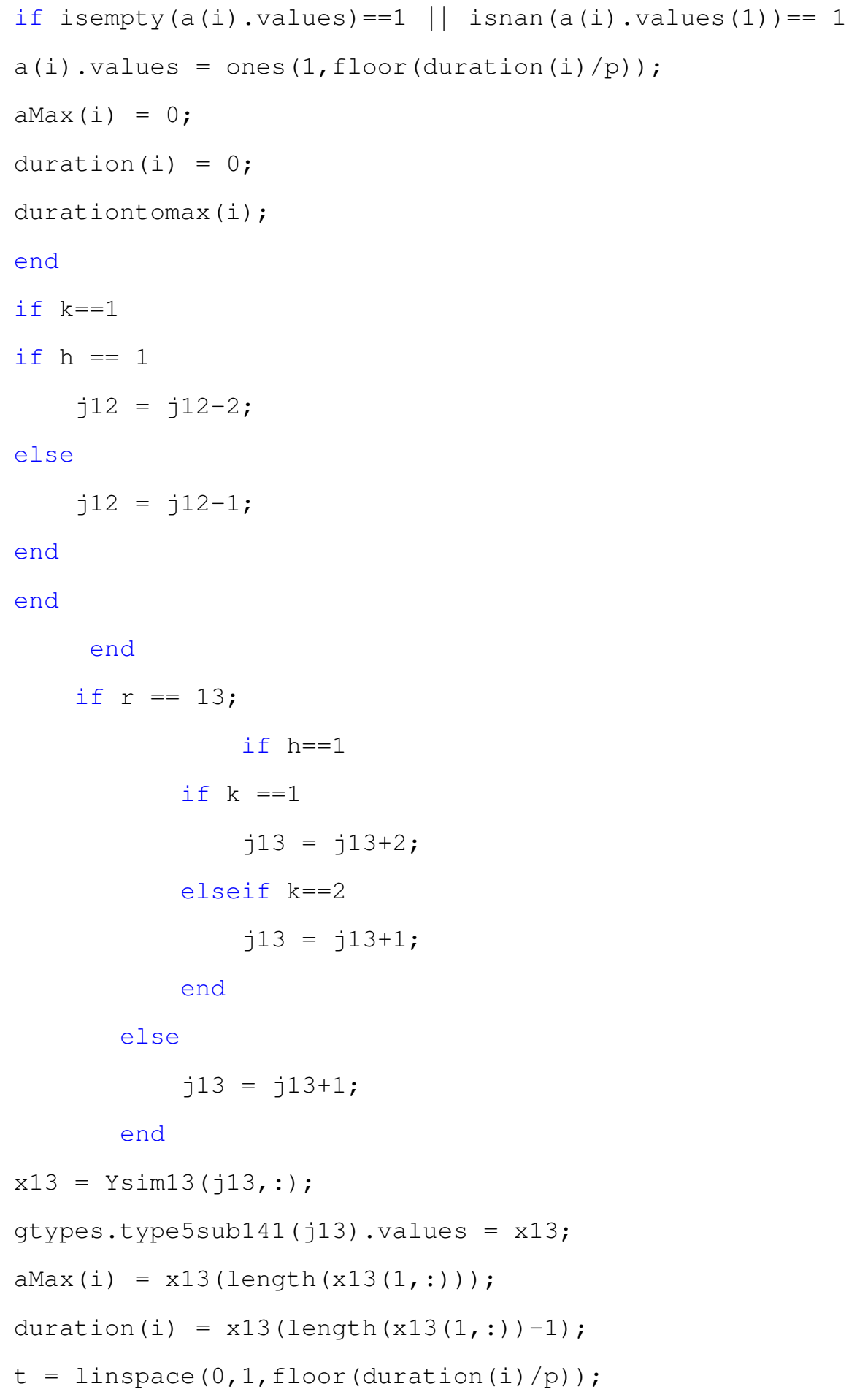




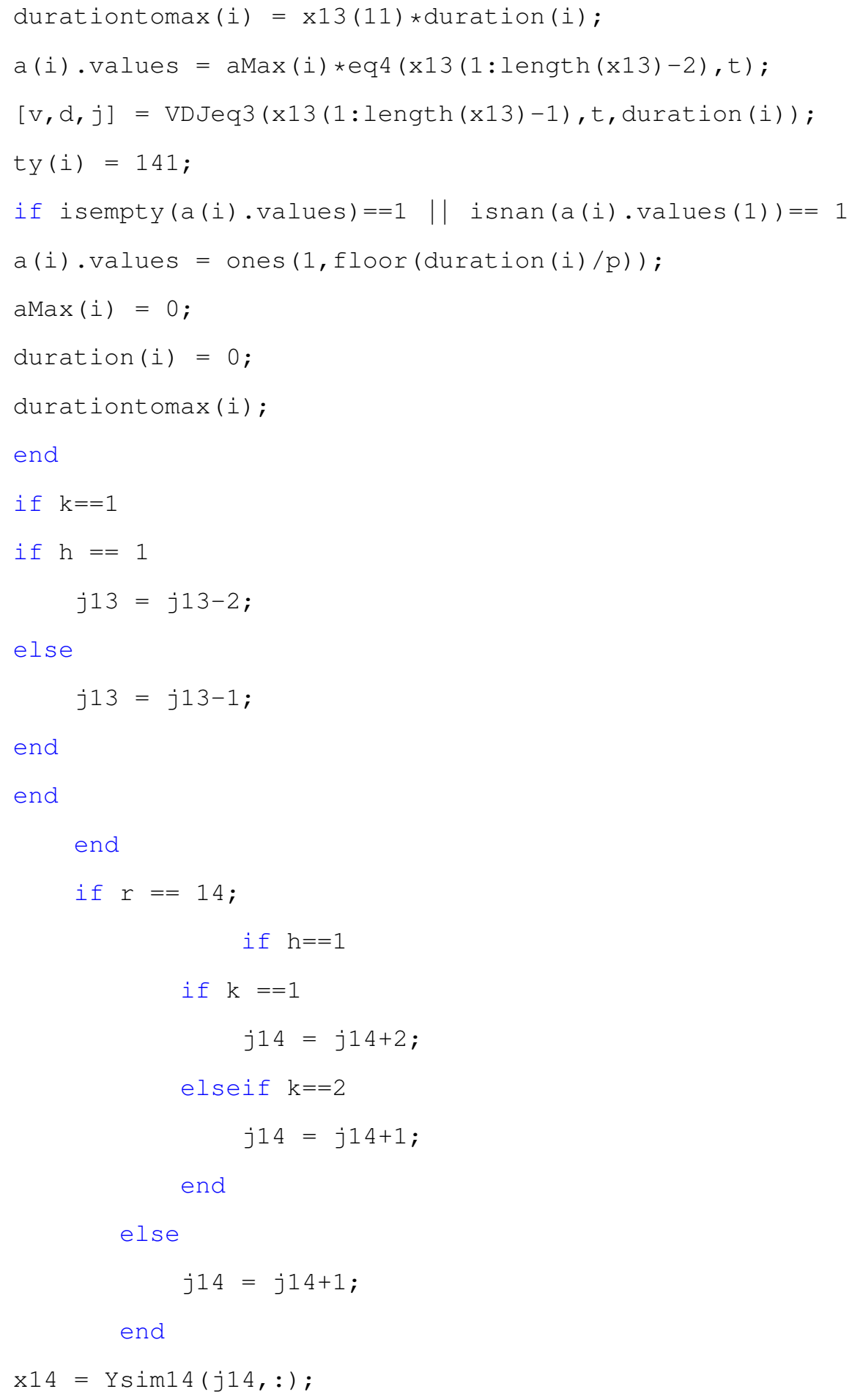




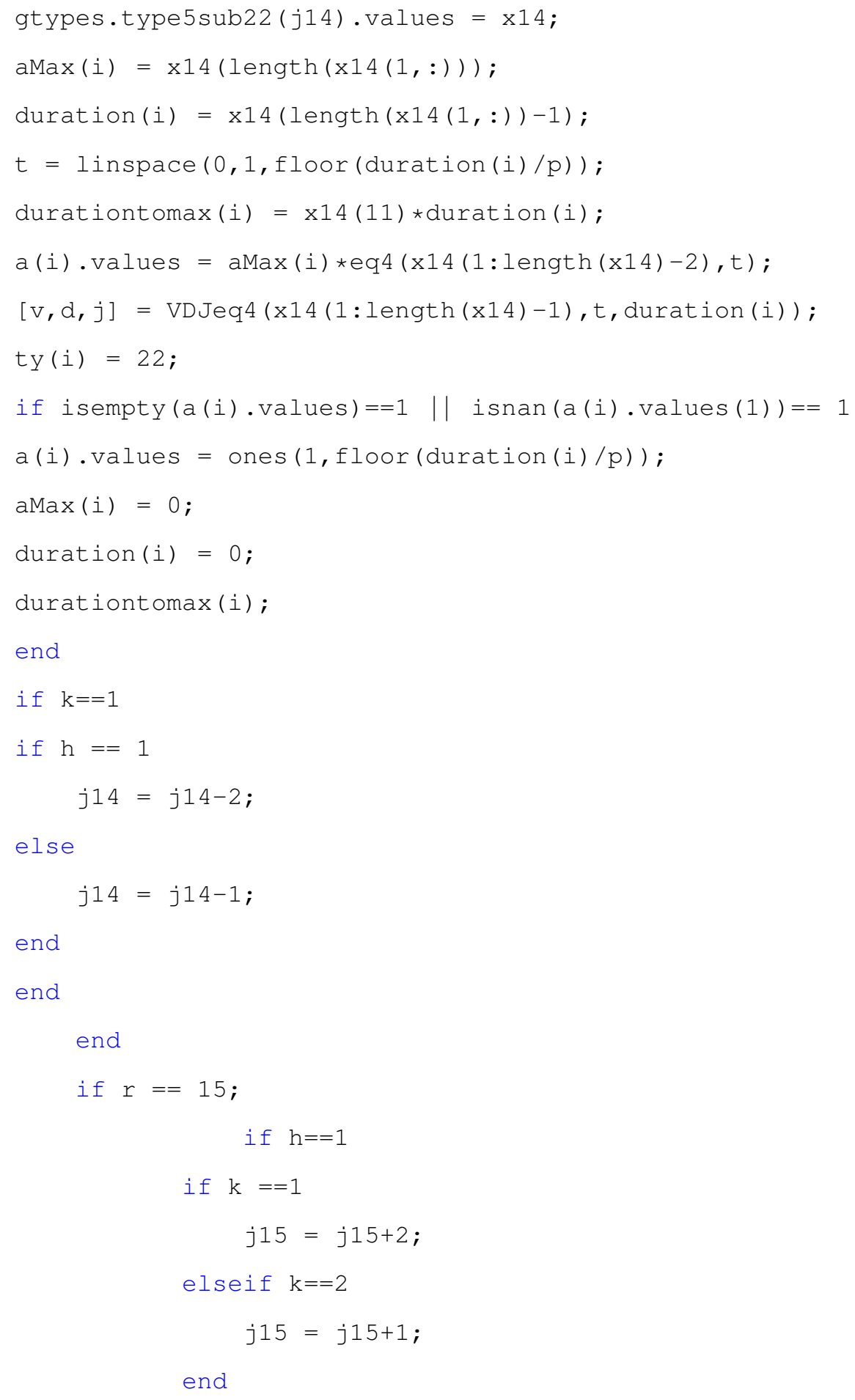




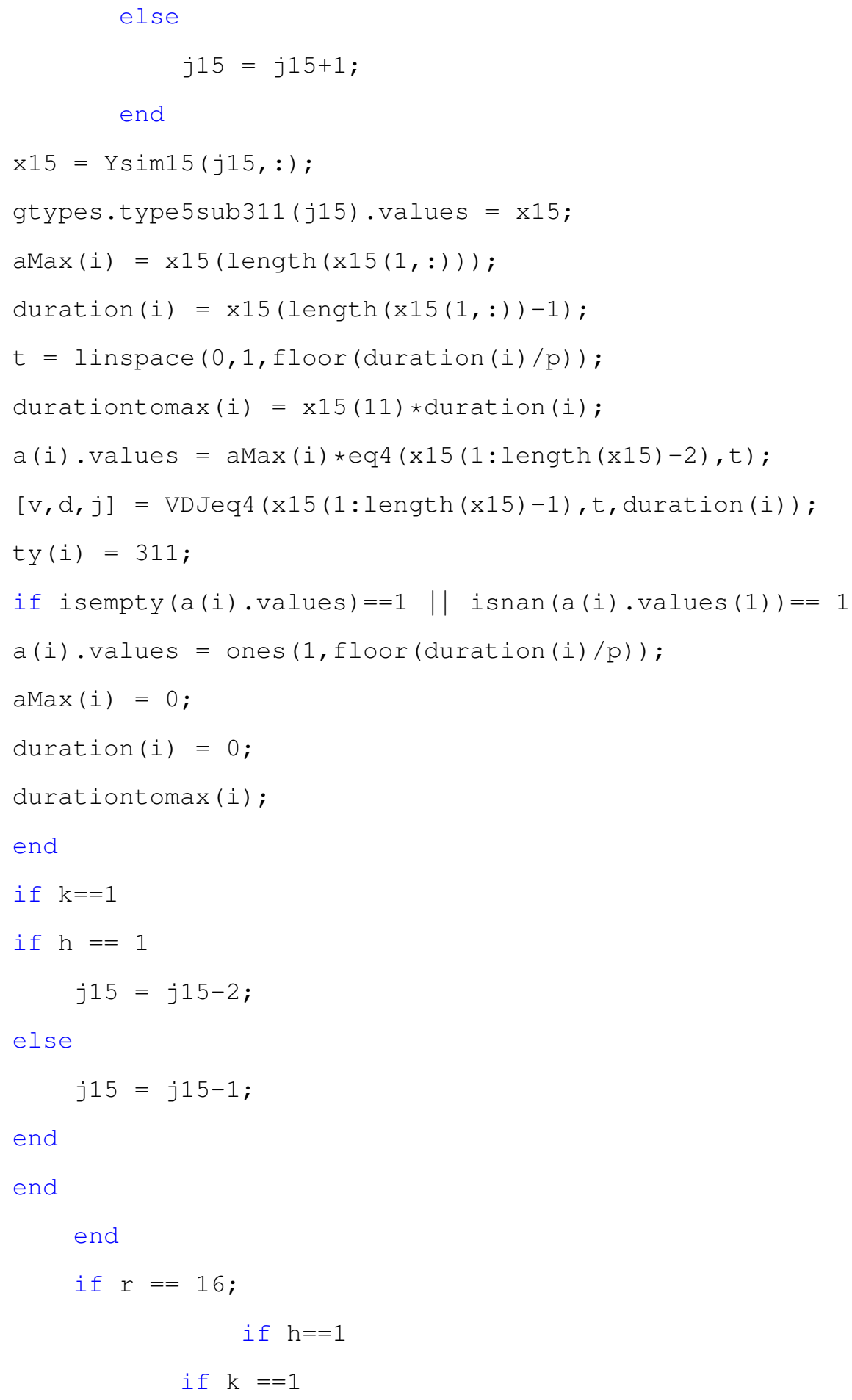




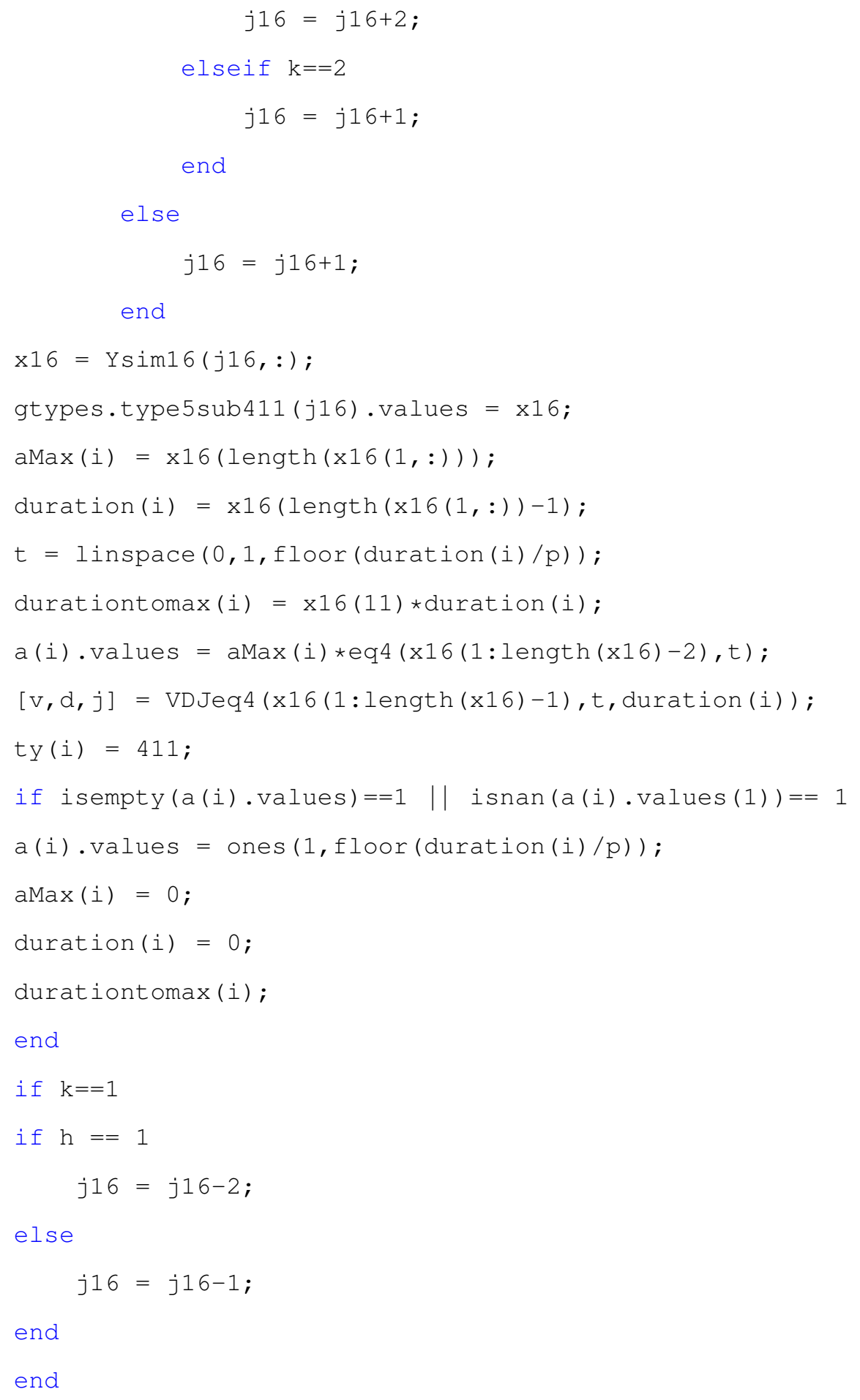


end

if $r==17$;

$$
\begin{aligned}
& \quad \text { if } h==1 \\
& \text { if } k==1 \\
& j 17=j 17+2 ; \\
& \text { elseif } k==2 \\
& j 17=j 17+1 ; \\
& \text { end }
\end{aligned}
$$

else

$$
j 17=j 17+1 ;
$$

end

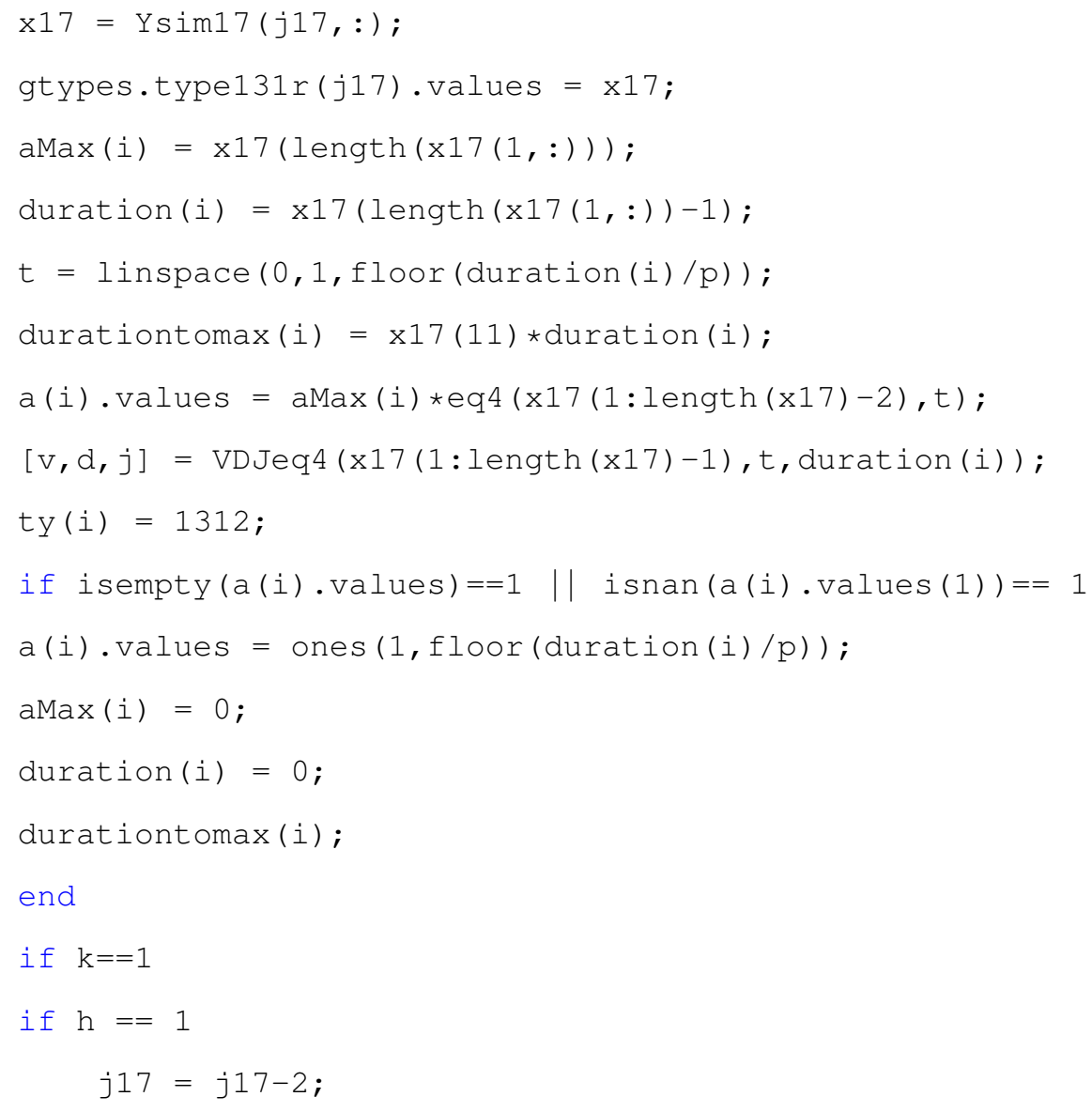




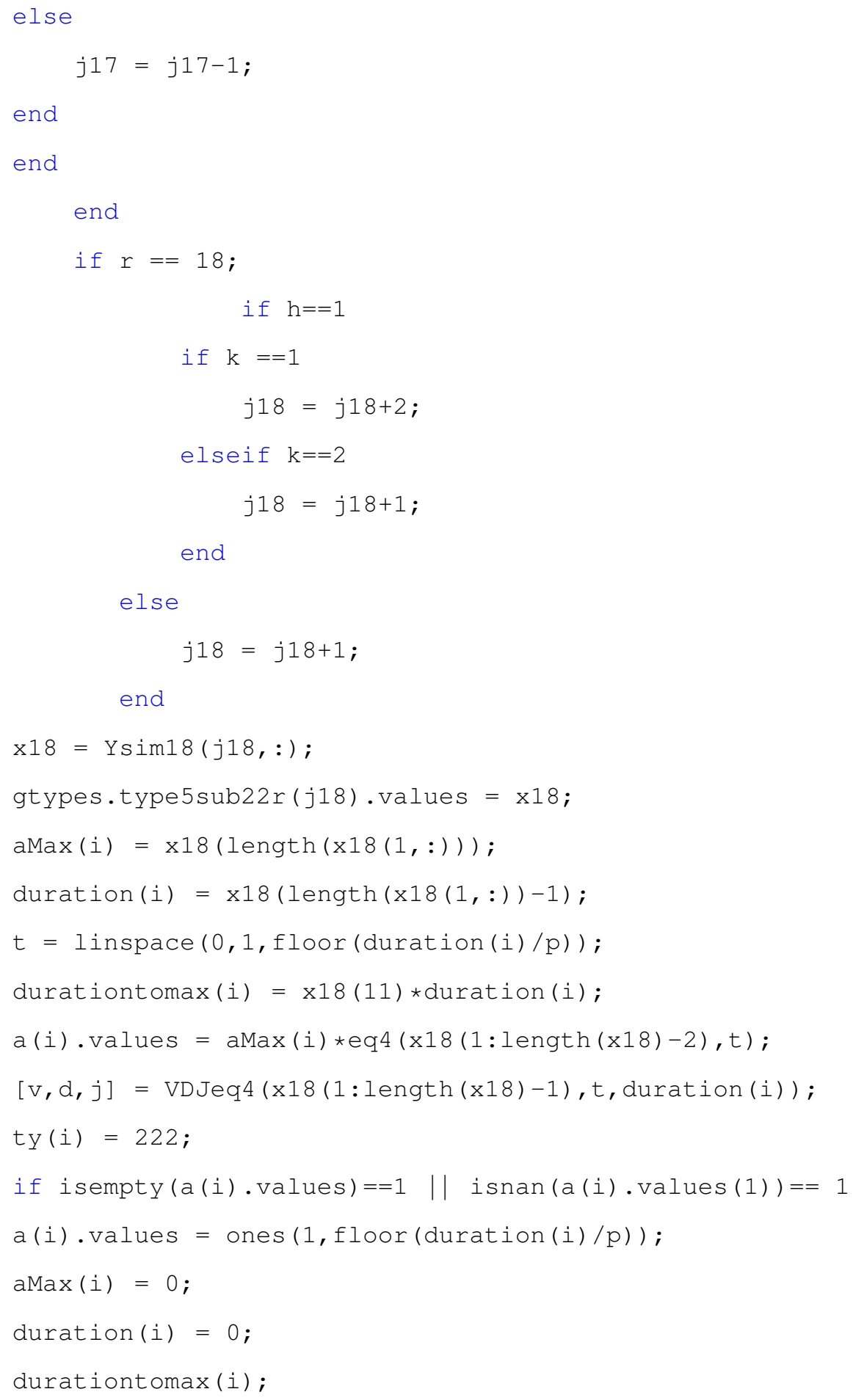




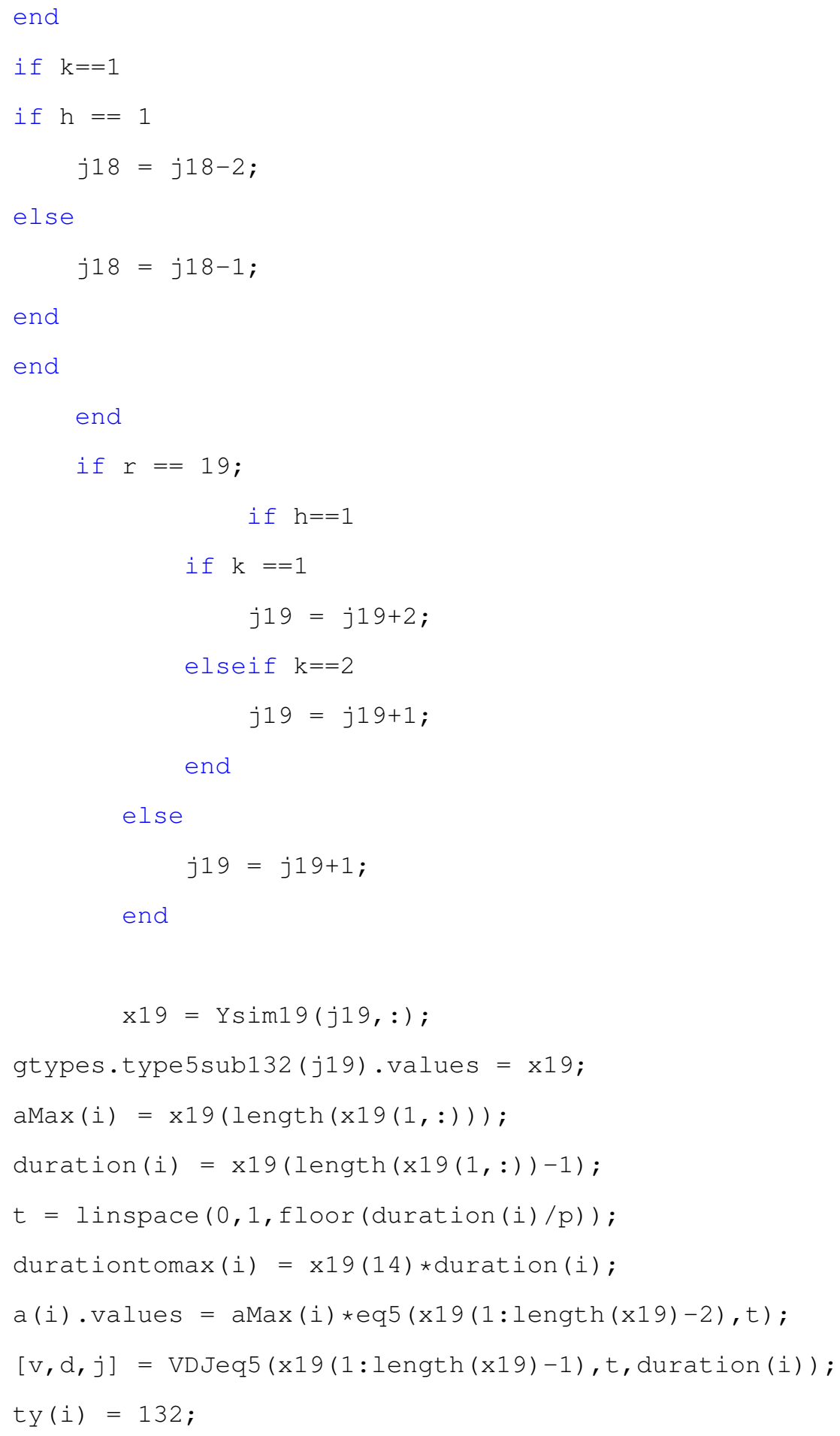




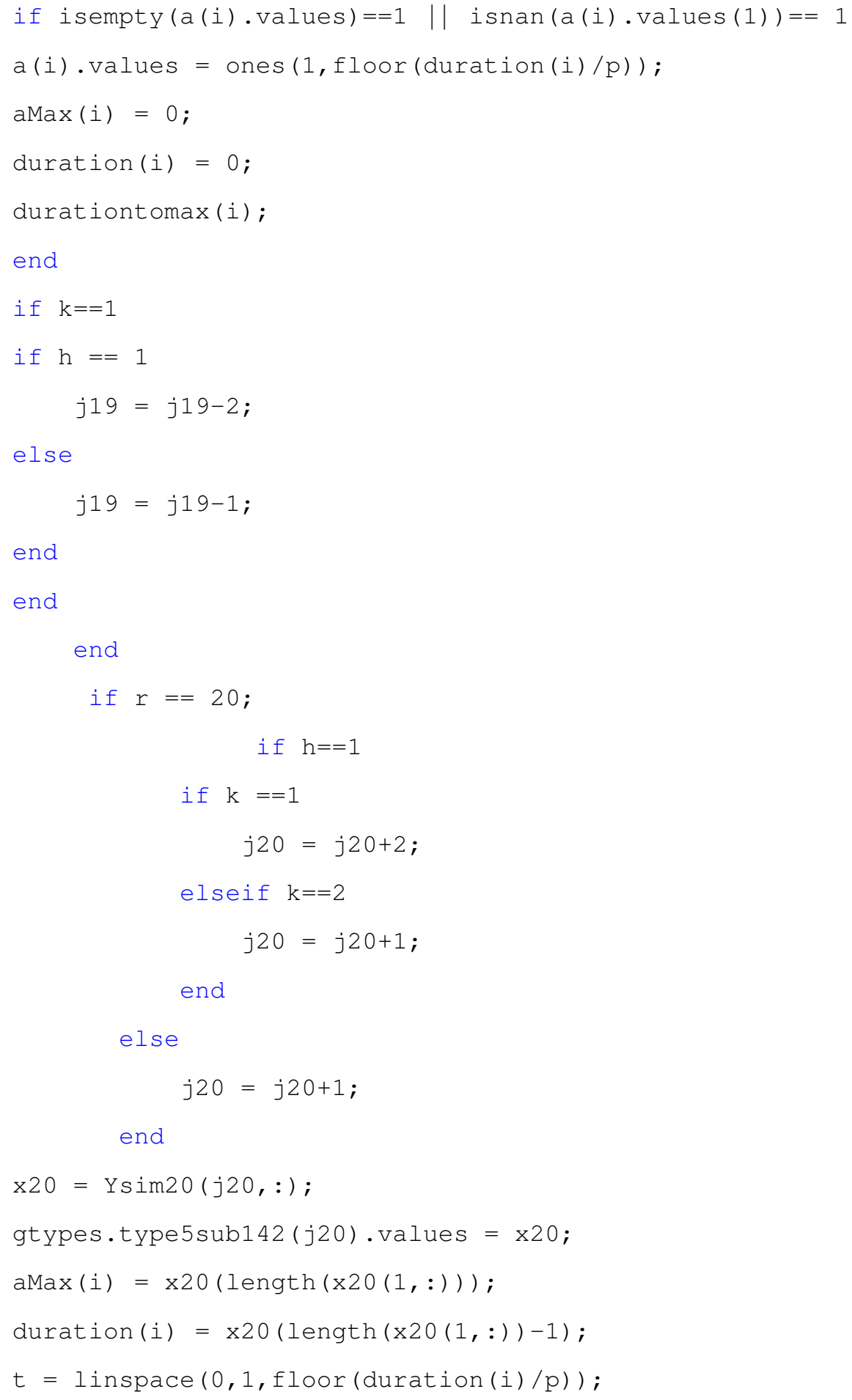




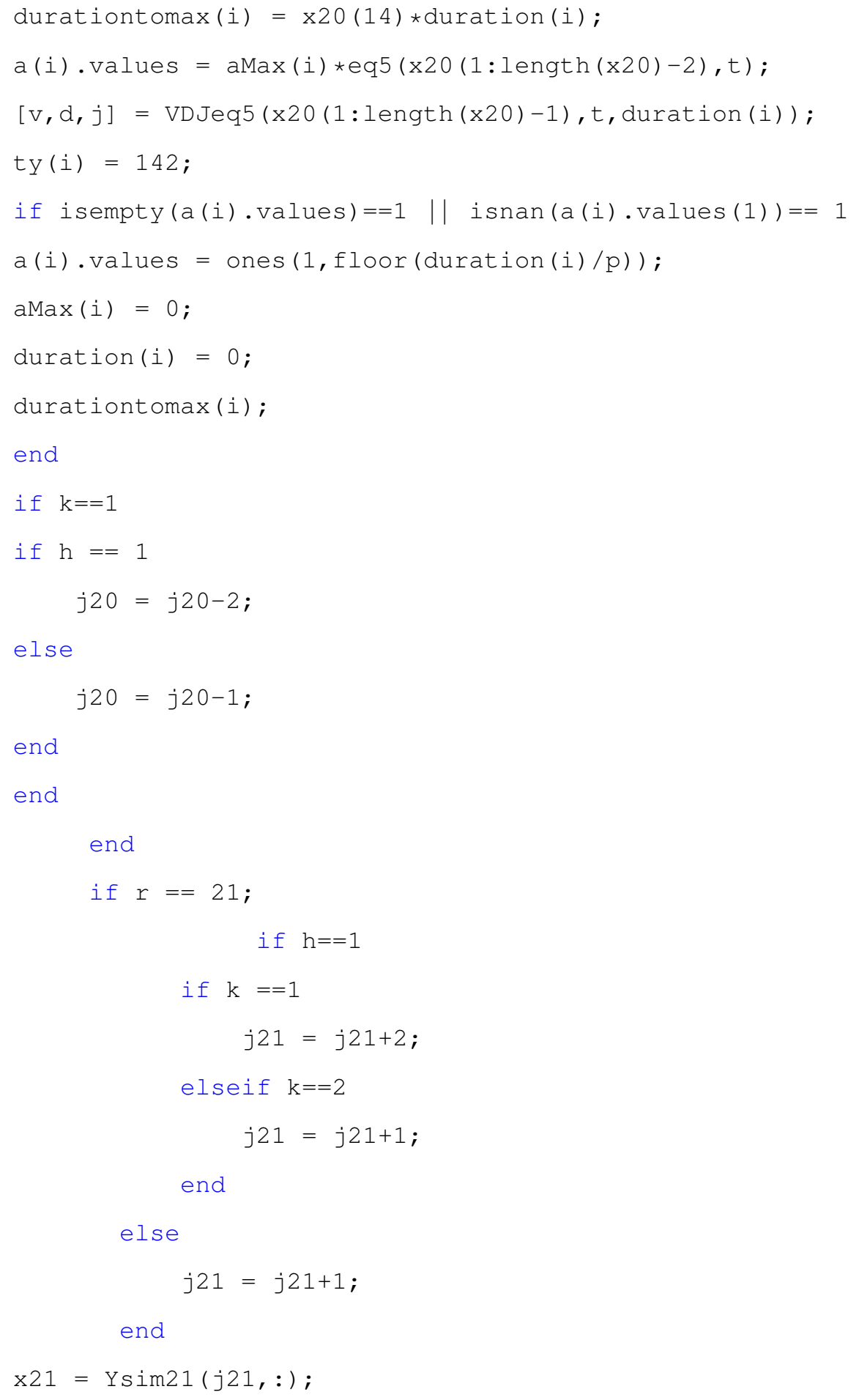




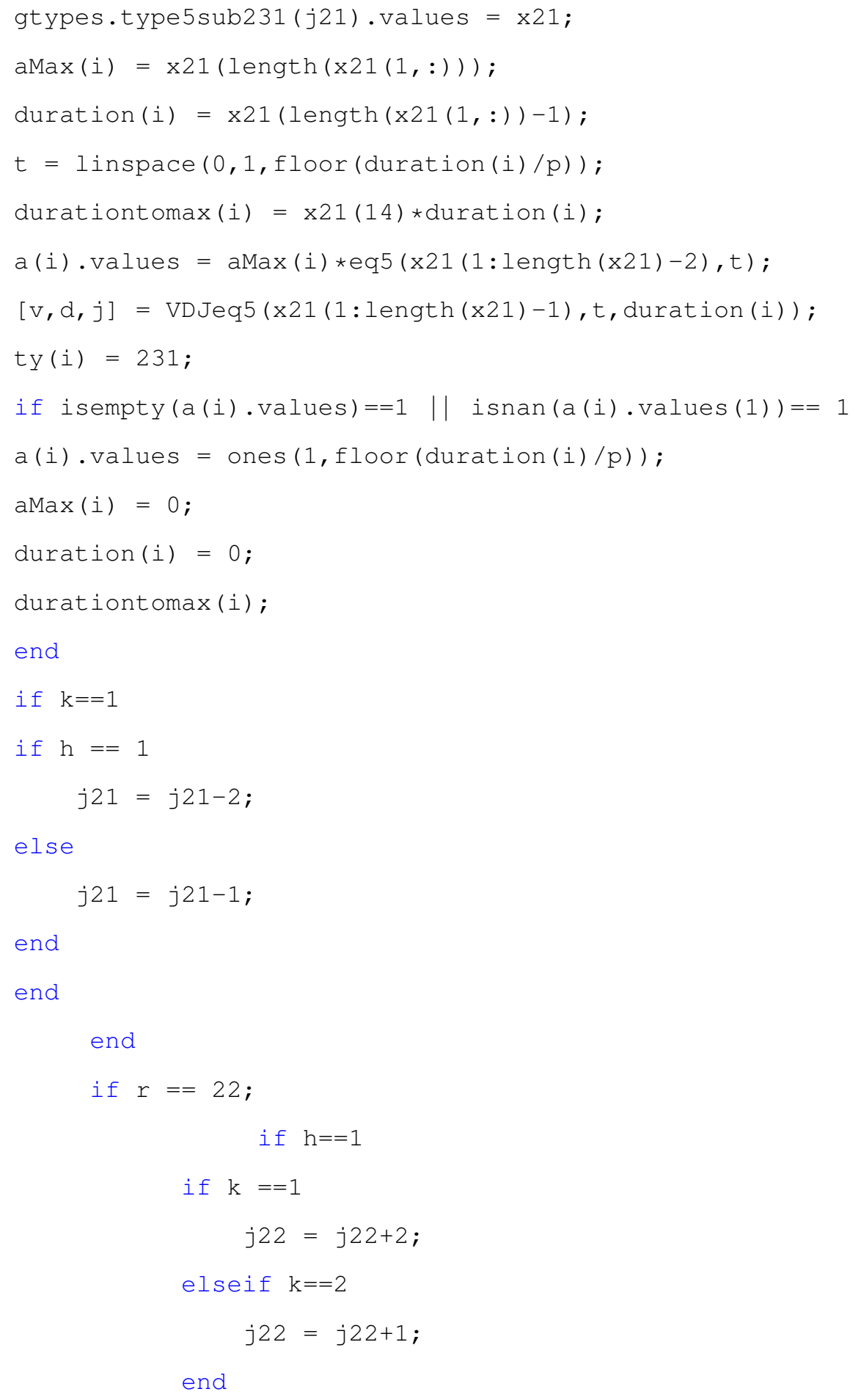




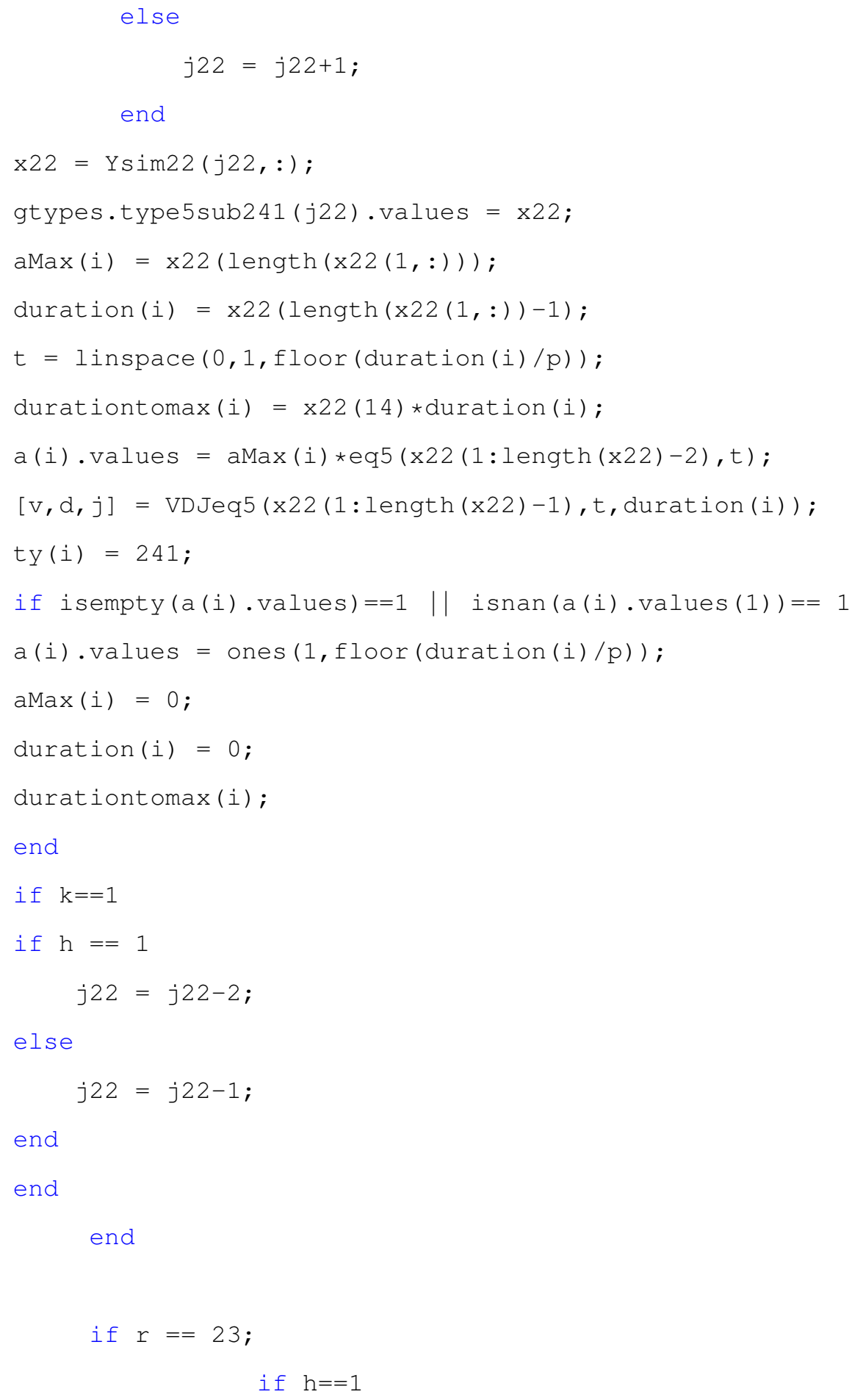




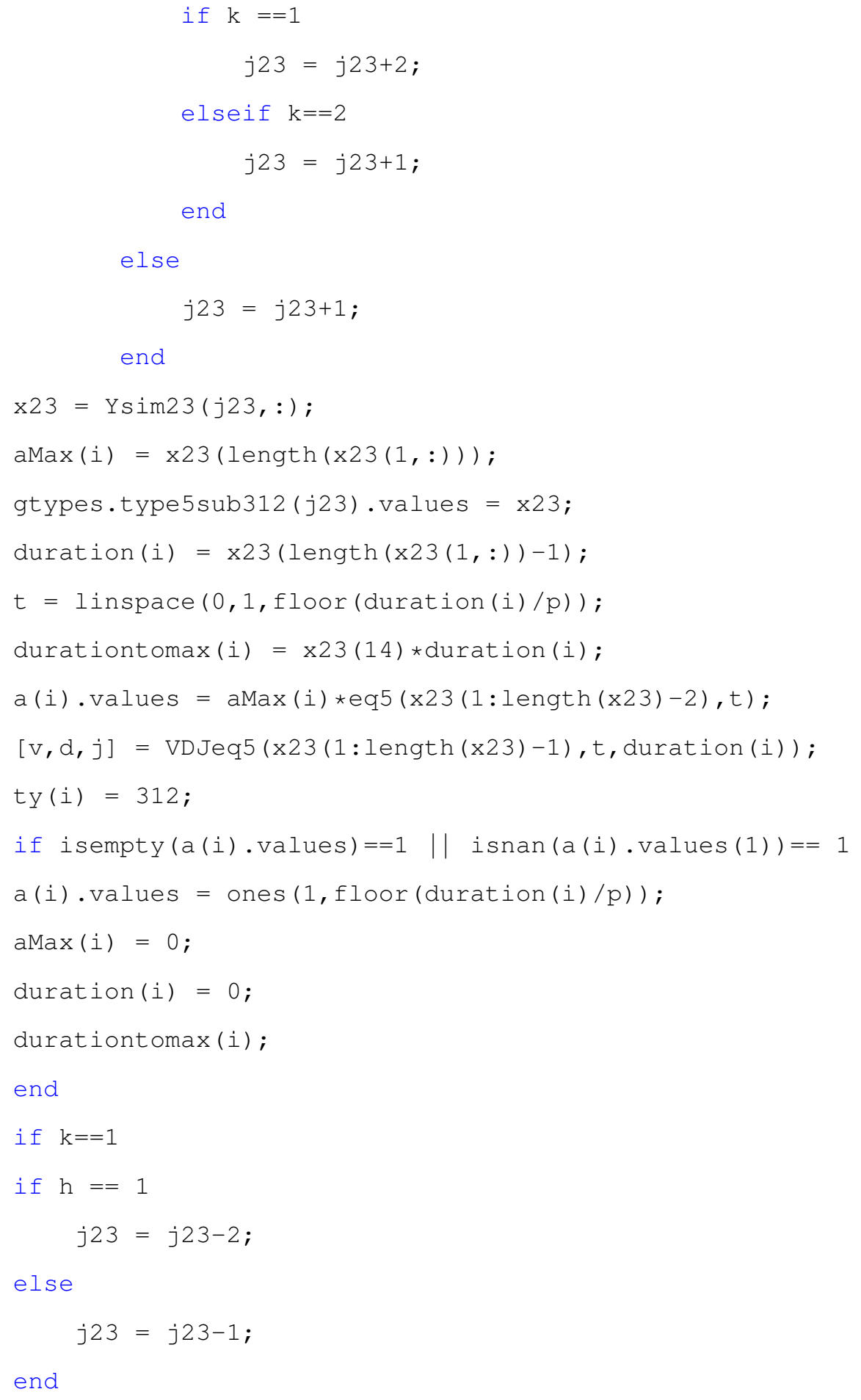




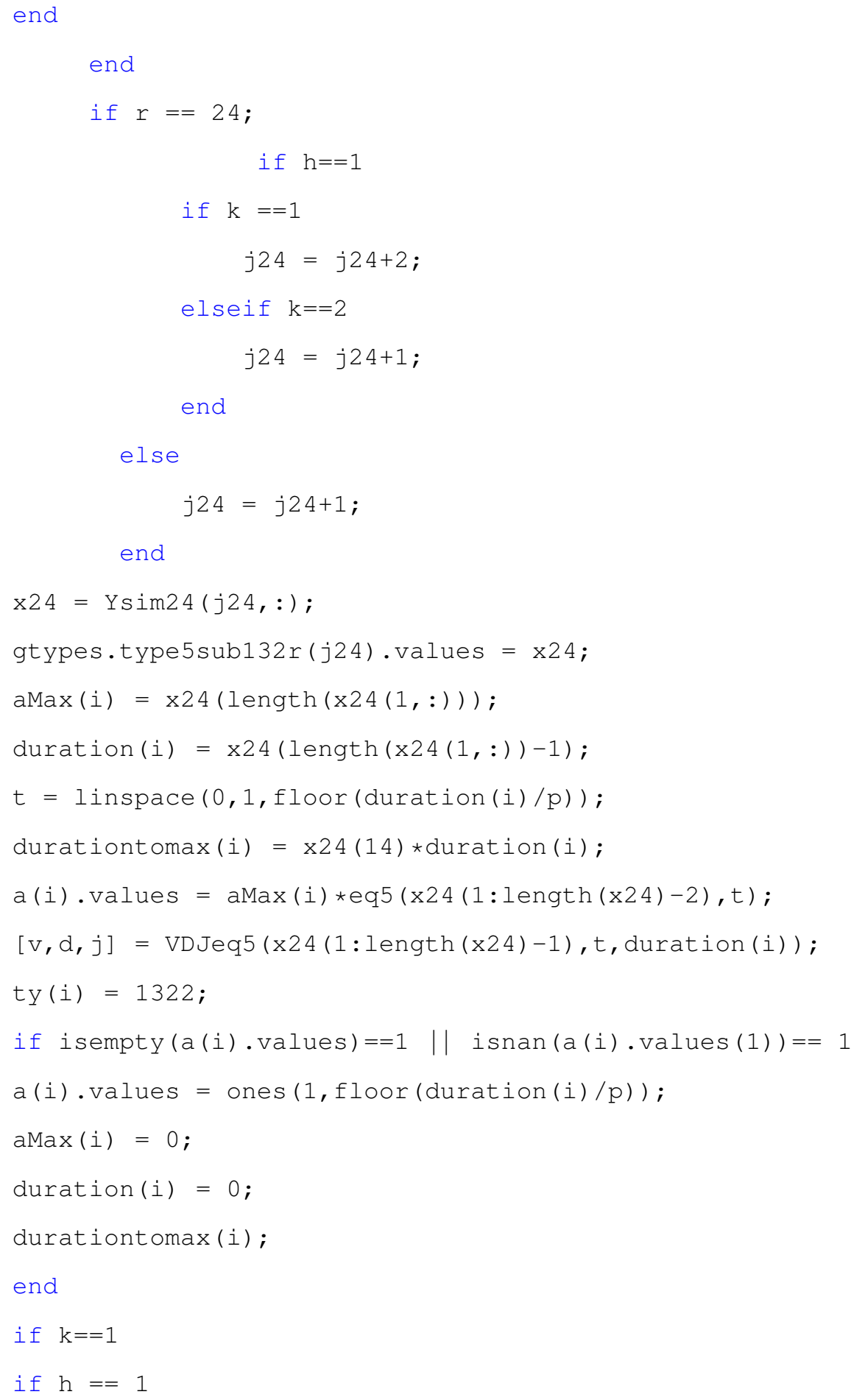




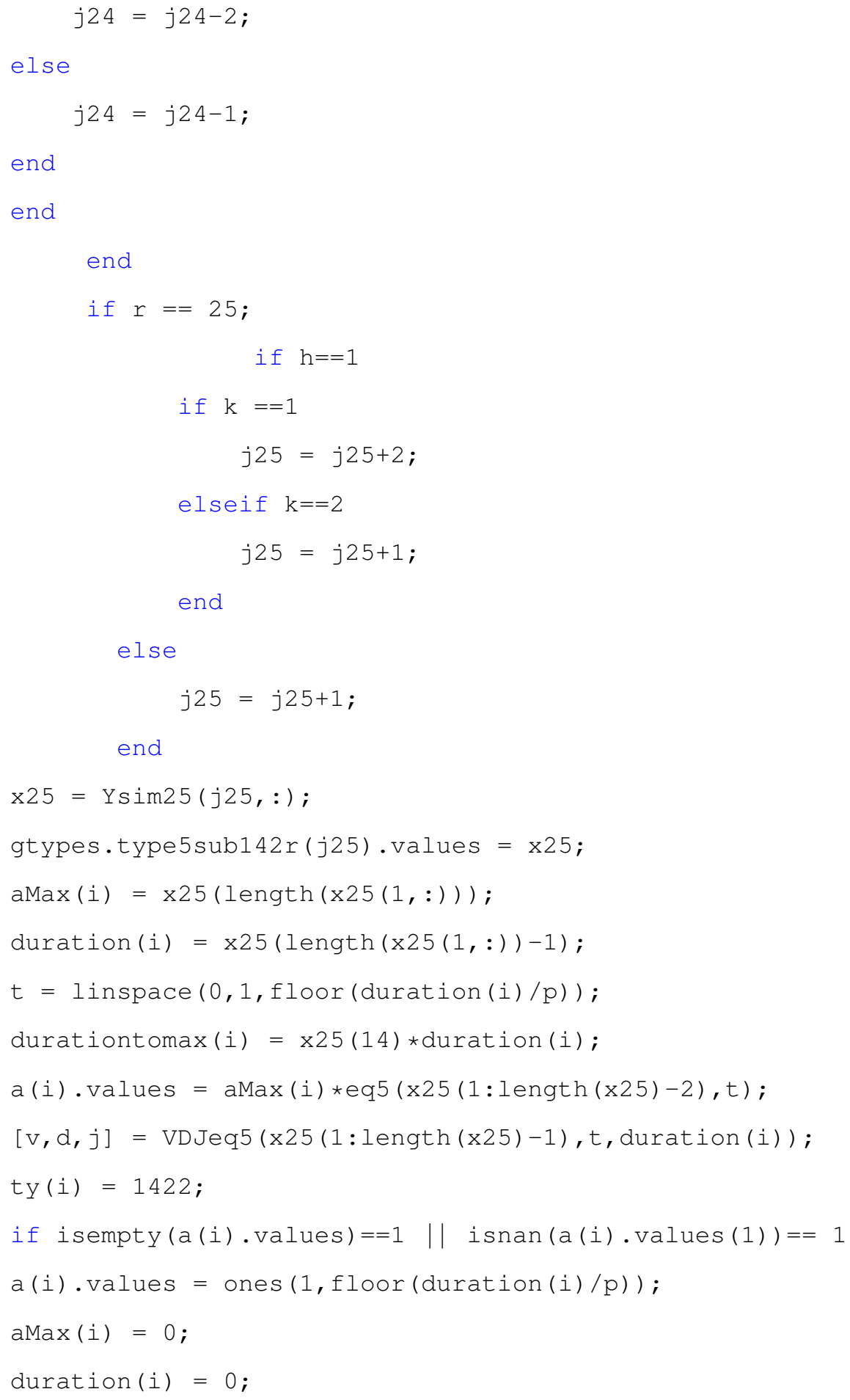




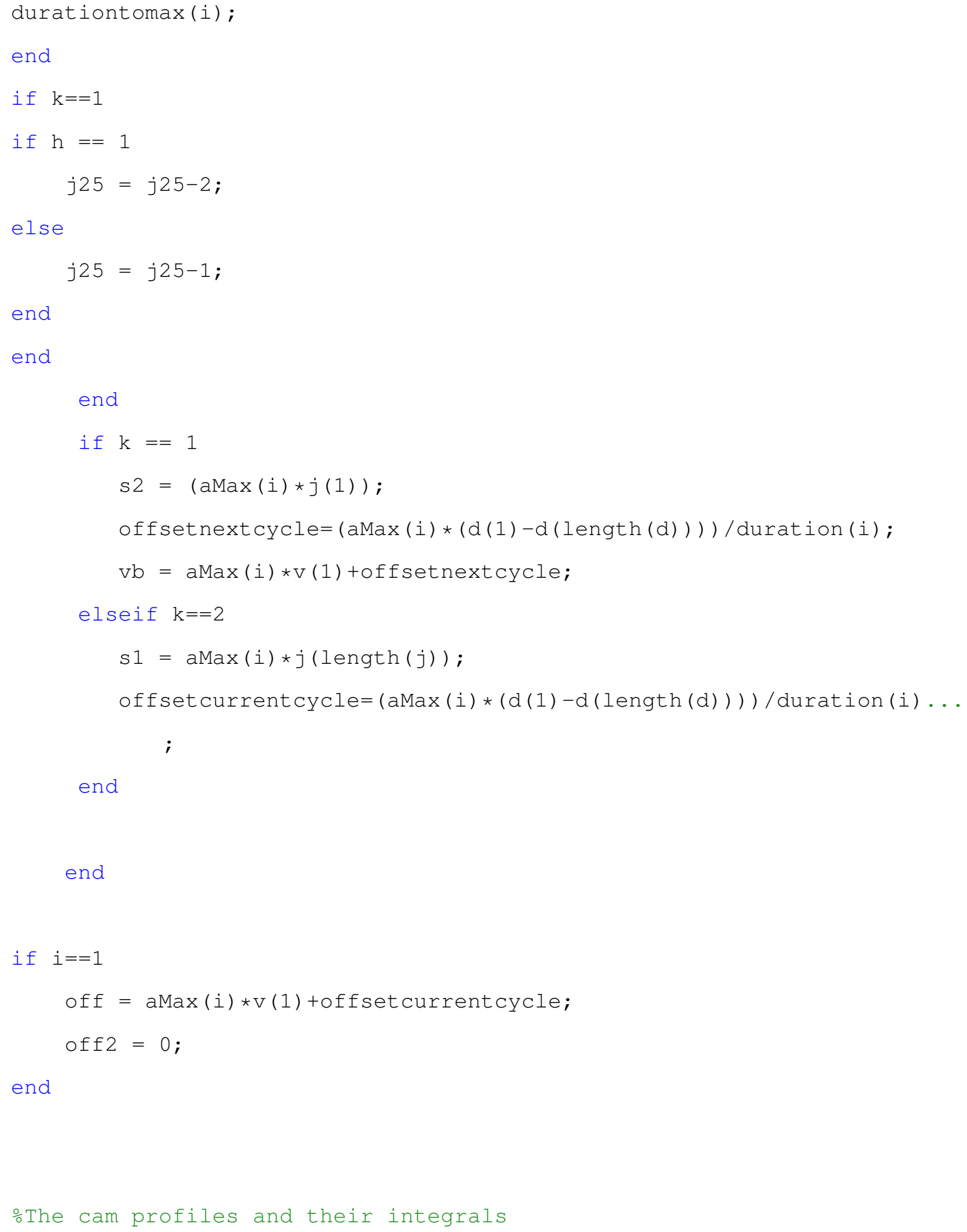




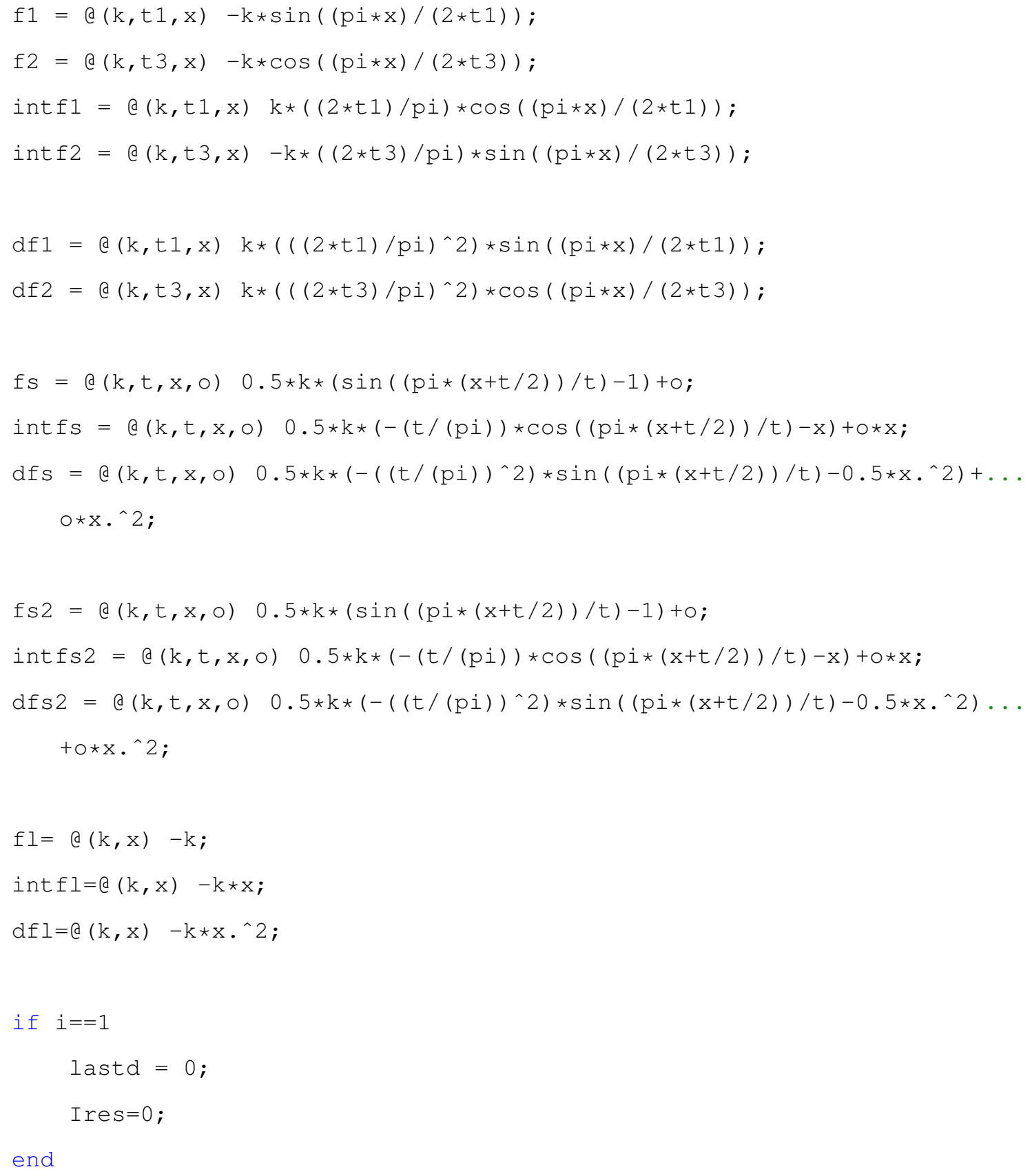


$93=@(x) \quad[\operatorname{dfl}(\operatorname{abs}(x(1)), \operatorname{abs}(x(2)))+(\operatorname{intfl}(\operatorname{abs}(x(1)),-(\operatorname{abs}(x(1)) \ldots$ $\star \mathrm{pi}) /(2 \star \mathrm{s} 1),-(\mathrm{abs}(\mathrm{x}(1)) \star \mathrm{pi}) /(2 \star \mathrm{s} 1))+\operatorname{aMax}(\mathrm{i}) \star \mathrm{v}($ length $(\mathrm{v}))+\mathrm{off}-\ldots$ $\operatorname{aMax}(i) \star v(1)-\ldots$

$\operatorname{intf1}(\operatorname{abs}(x(1)),-(\operatorname{abs}(x(1)) * \operatorname{pi}) /(2 * s 1), 0)-\operatorname{intfl}(\operatorname{abs}(x \ldots$ $(1)), 0)) * \operatorname{abs}(x(2))+\ldots$ $\operatorname{dfl}(\operatorname{abs}(x(1)),-(\operatorname{abs}(x(1)) * \mathrm{pi}) /(2 * \mathrm{~s} 1),-(\mathrm{abs}(\mathrm{x}(1)) \star \mathrm{pi}) \ldots$ $/(2 * \mathrm{~s} 1))+(\operatorname{aMax}(i) \star v($ length $(\mathrm{v}))+\ldots$

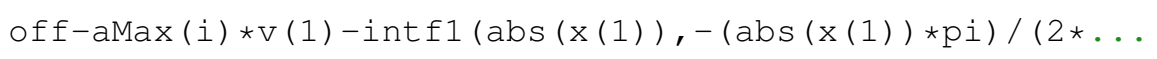




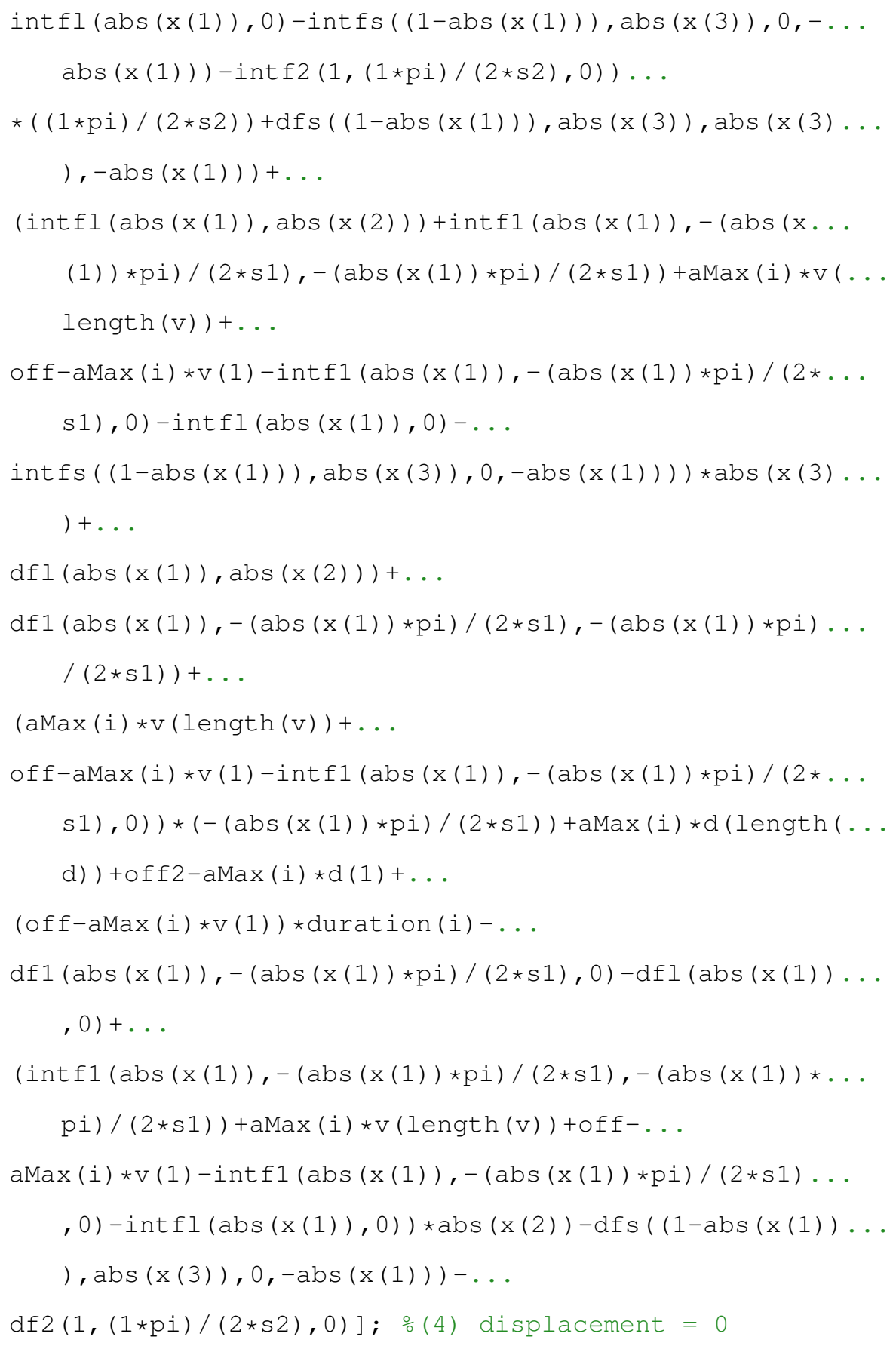




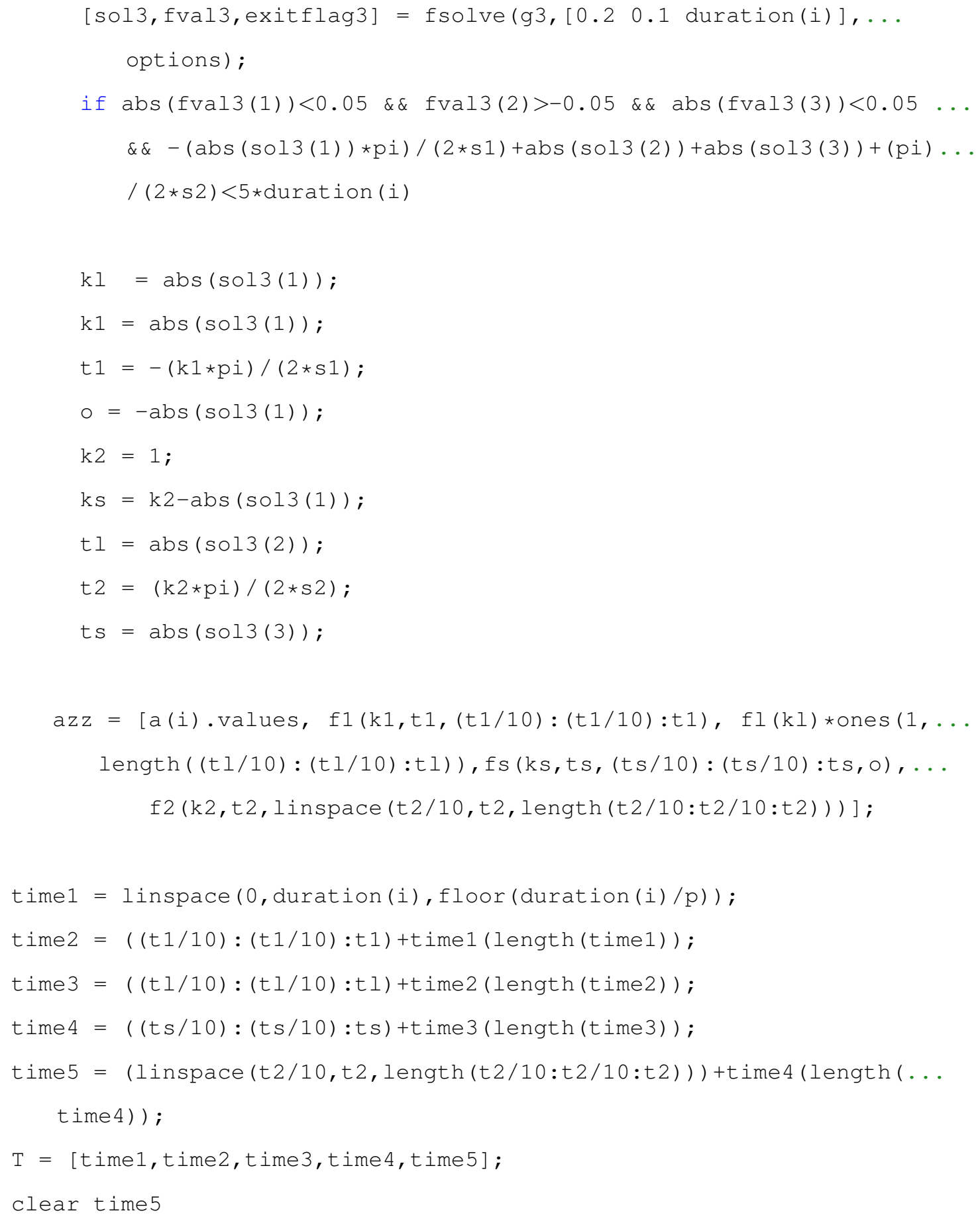




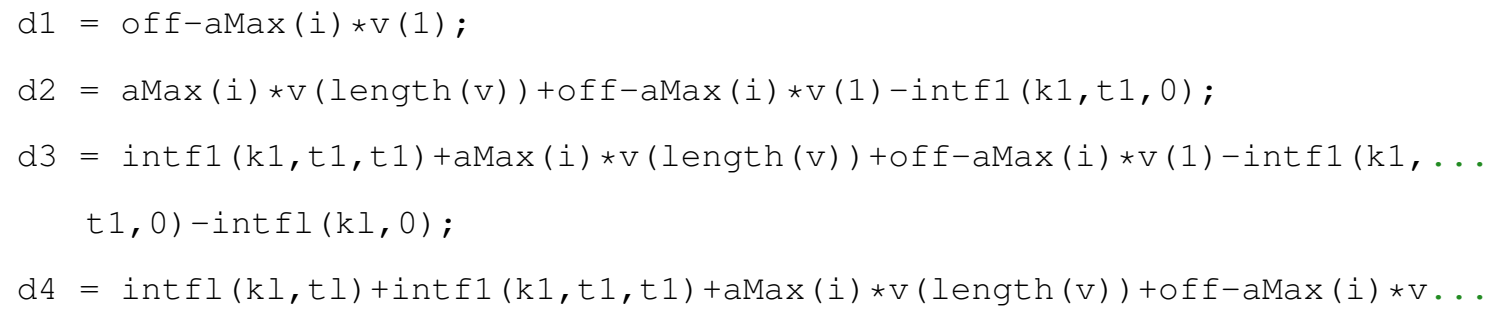

$\mathrm{vzz}=[\operatorname{aMax}(i) \star v+d 1, \operatorname{intfl}(k 1, t 1,(t 1 / 10):(t 1 / 10): t 1)+d 2, \operatorname{intfl}(k 1,((\ldots$ tl/10):(tl/10):tl)) +d3, intfs(ks,ts, (ts/10):(ts/10):ts,o)+d4,... intf2 (k2, t2, linspace (t2/10,t2, length (t2/10:t2/10:t2))) +d5 ] ;

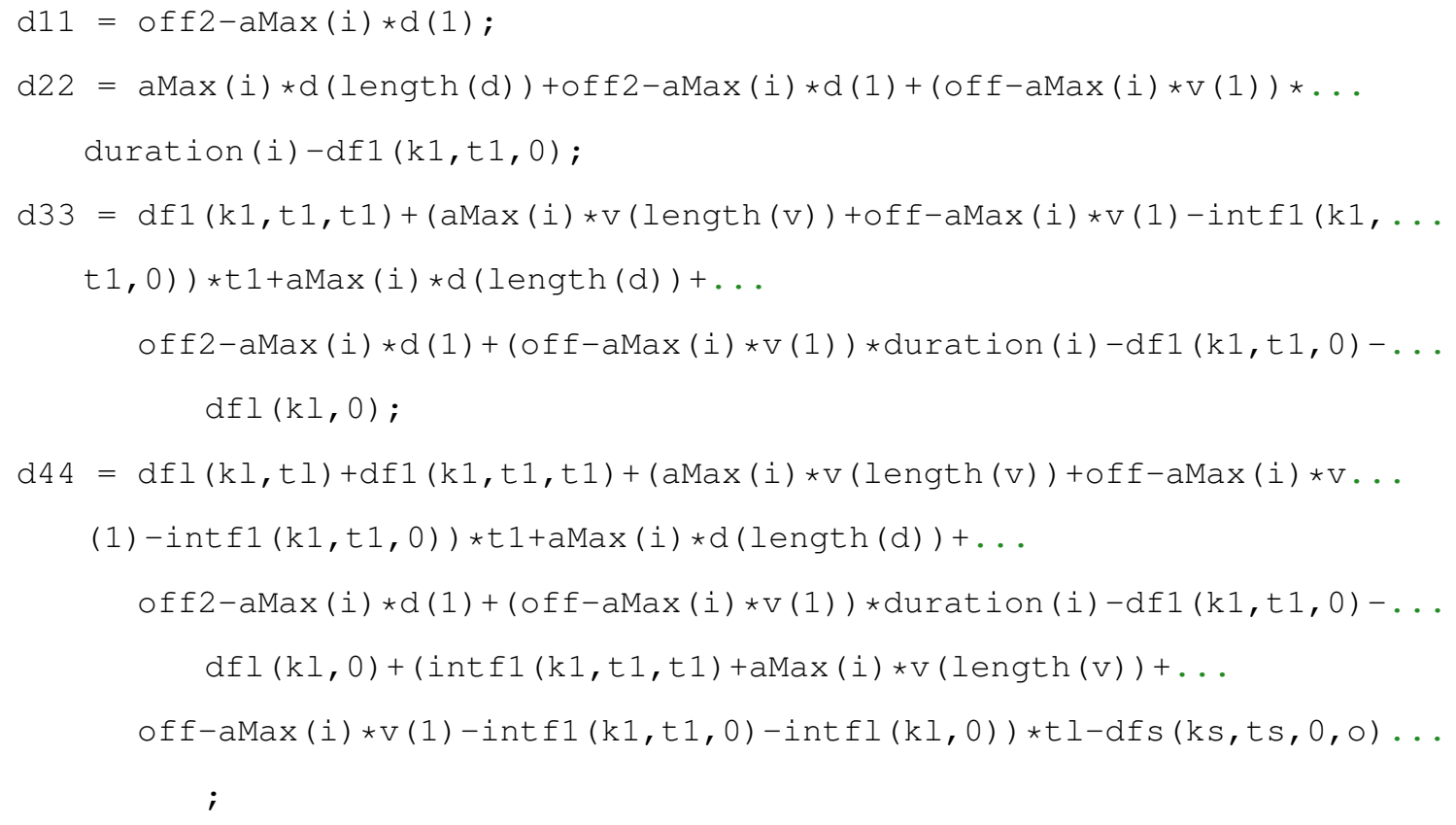




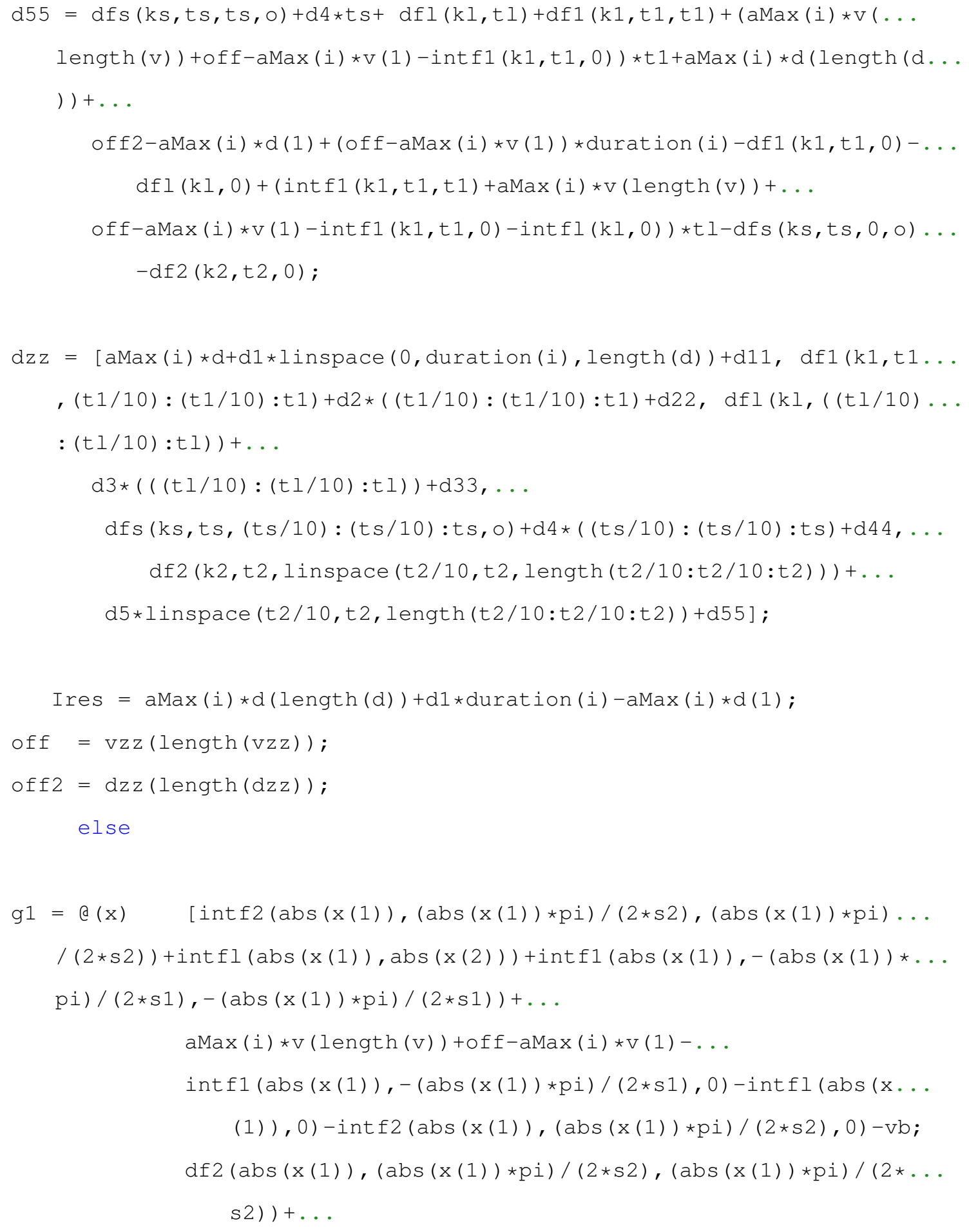




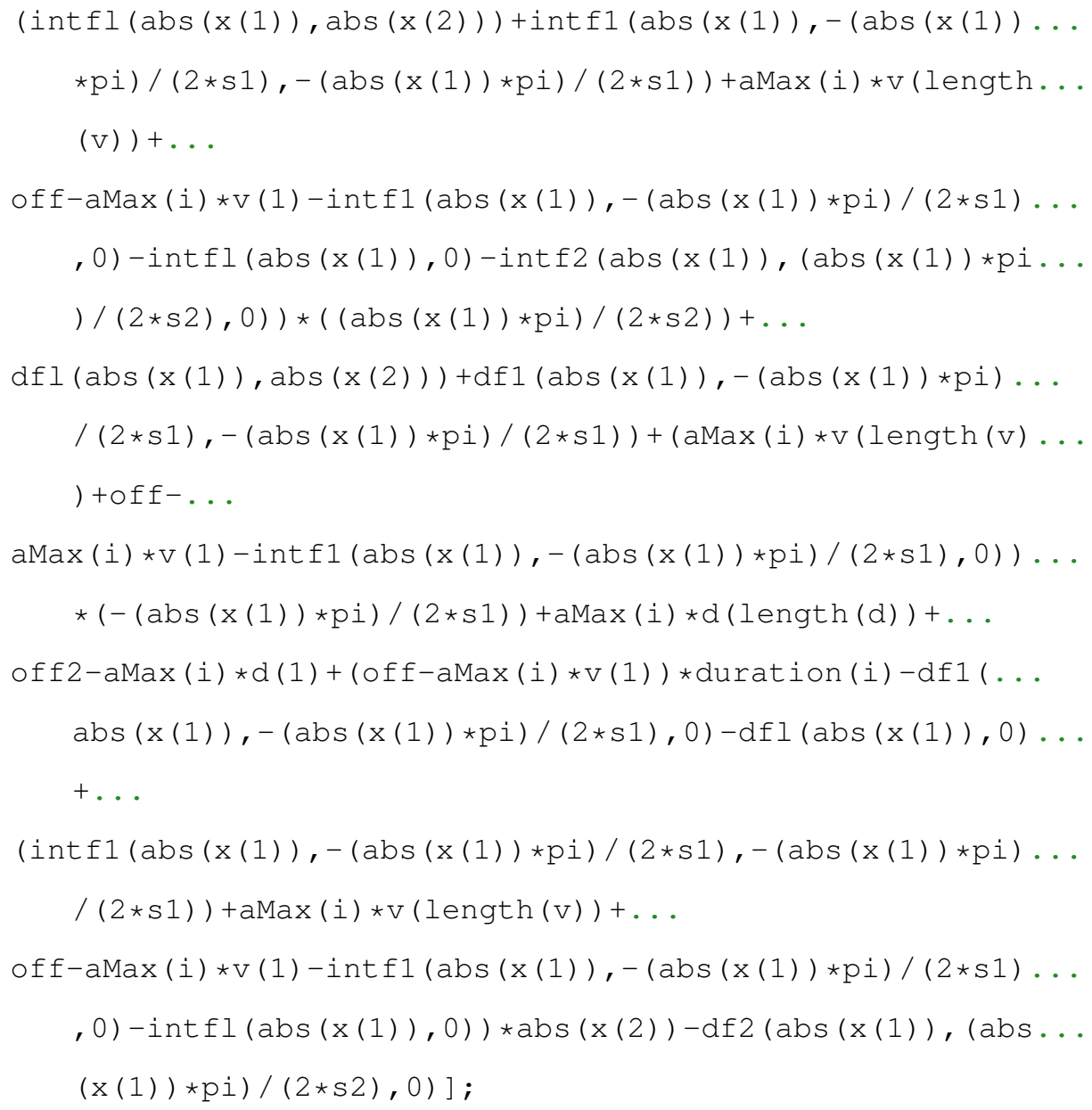




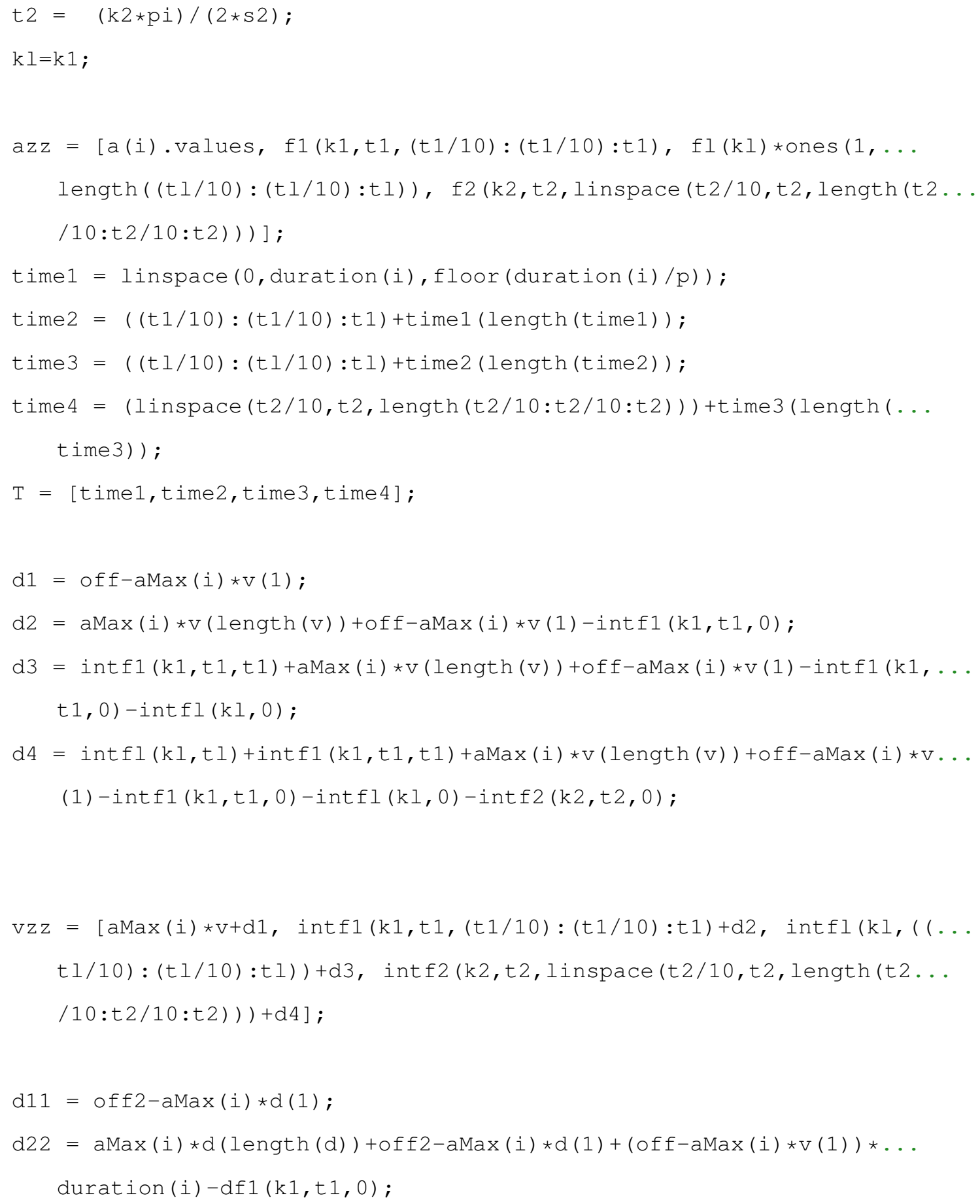




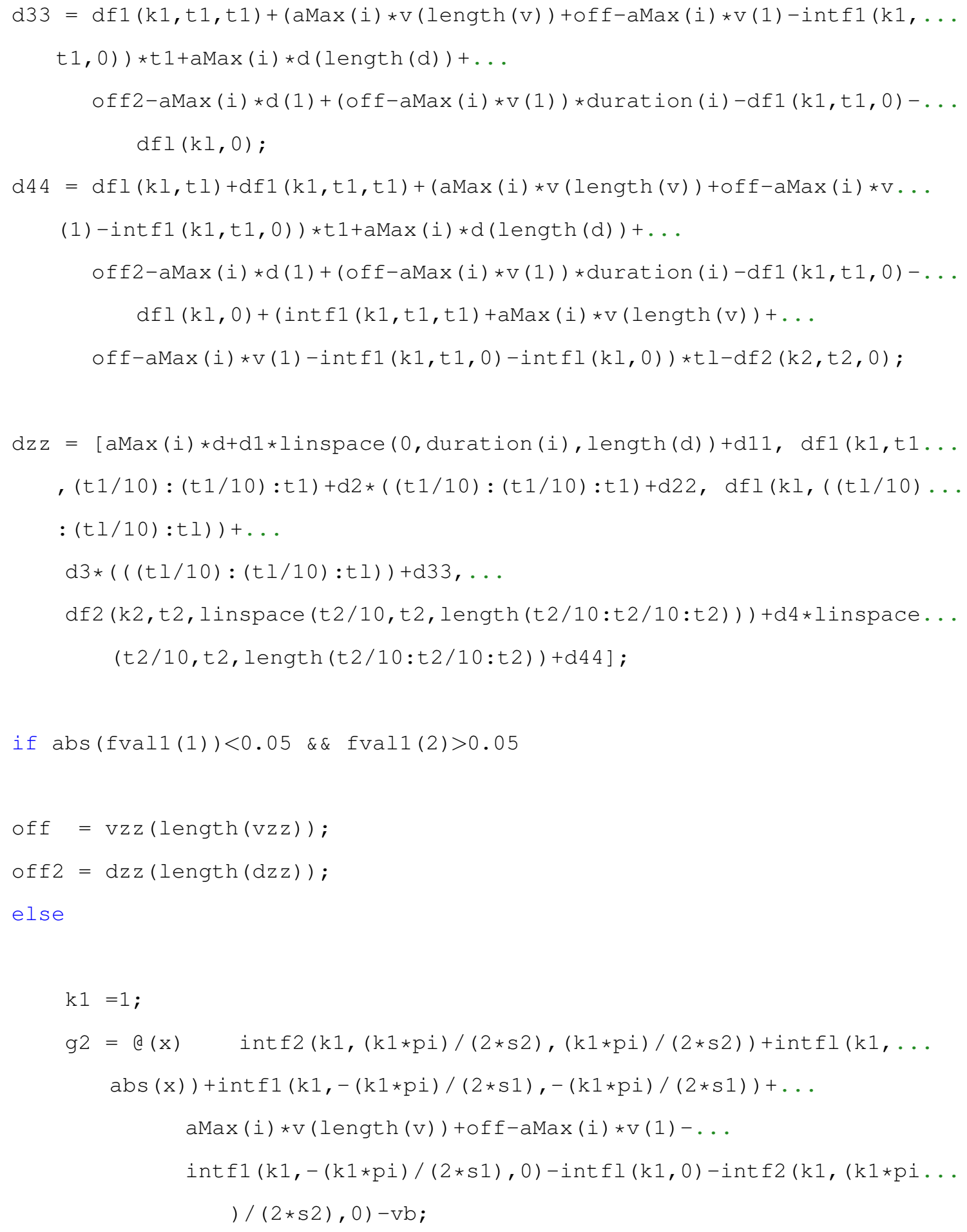




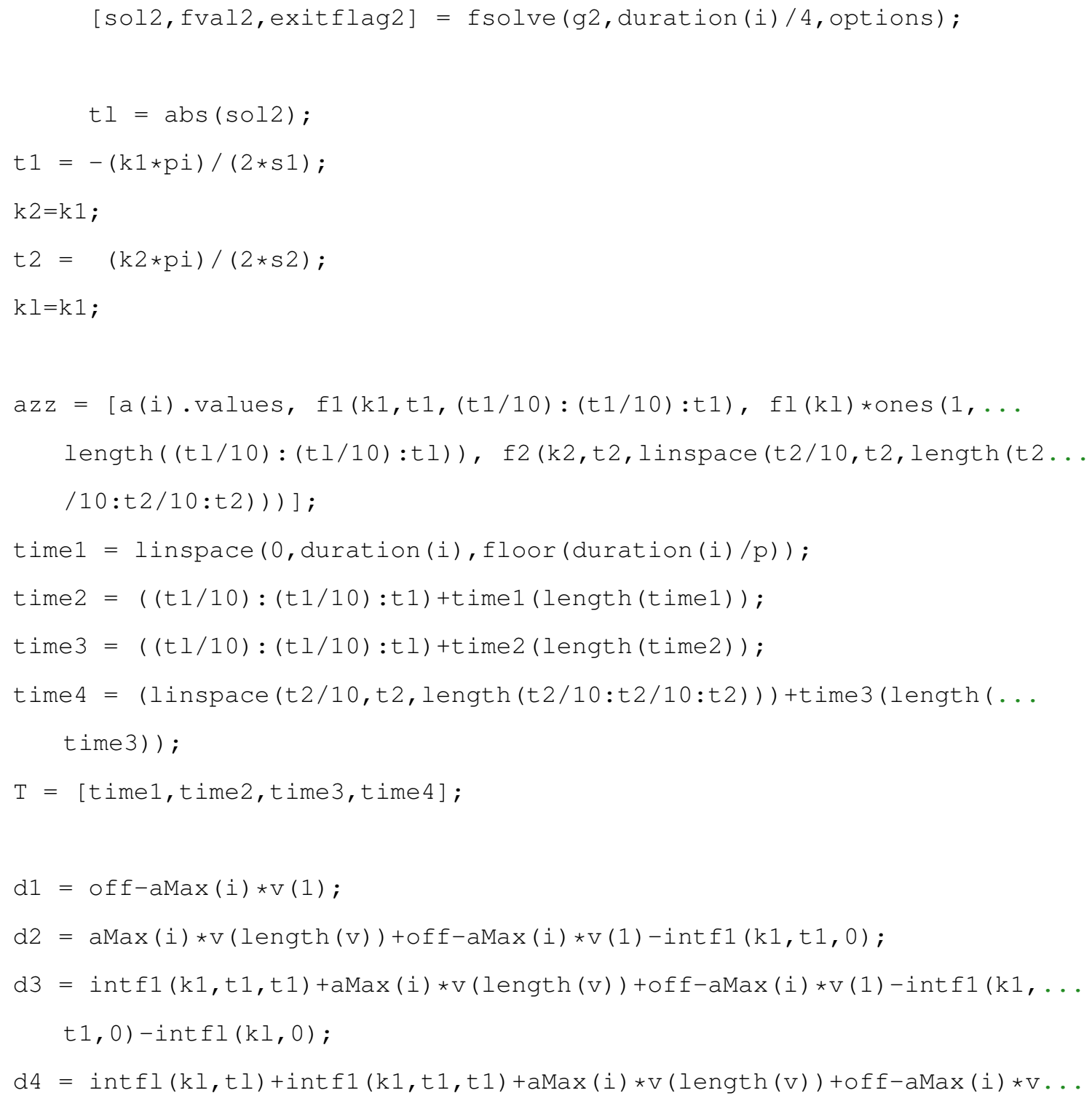




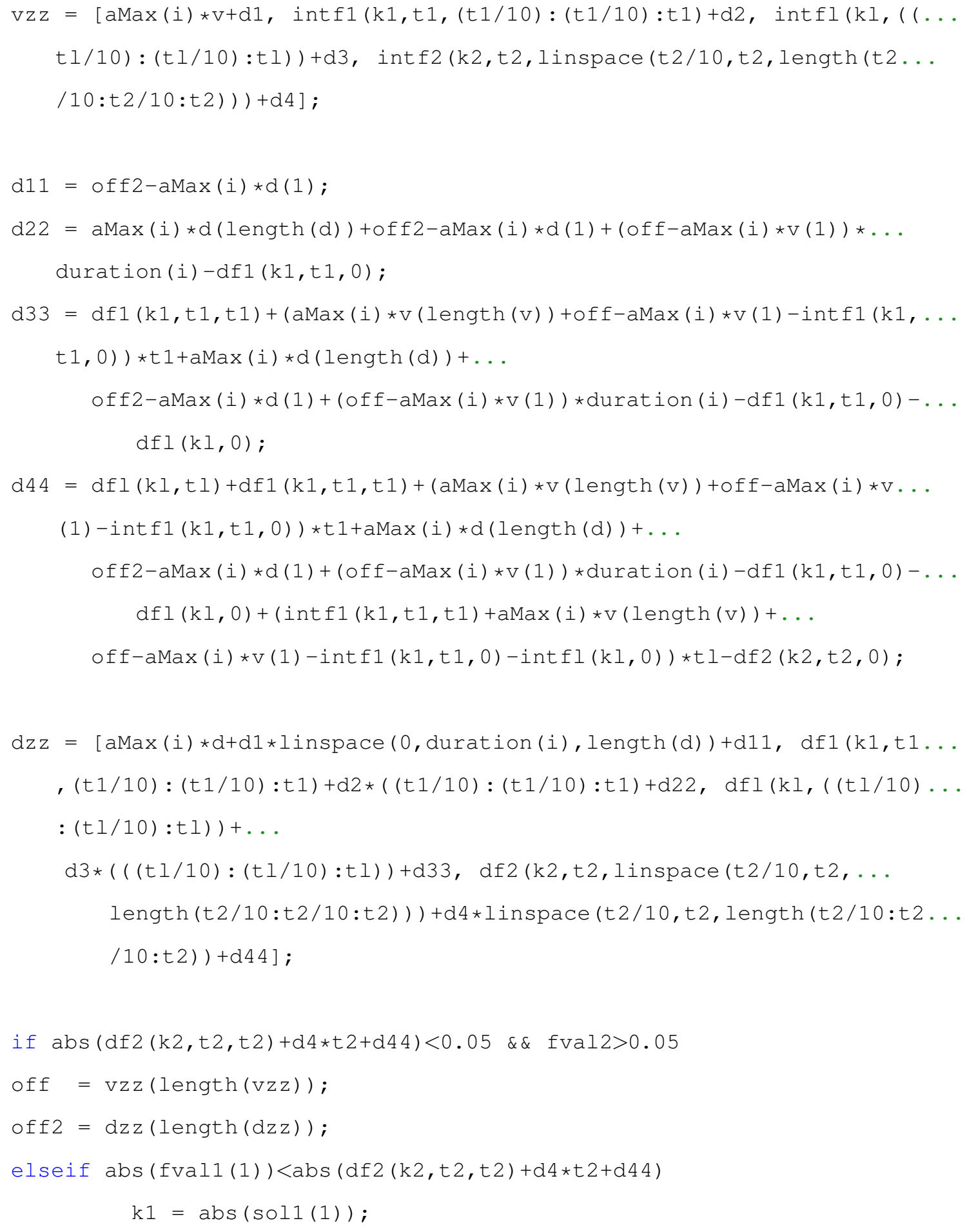




$$
\text { tI = abs (soll(2)); }
$$

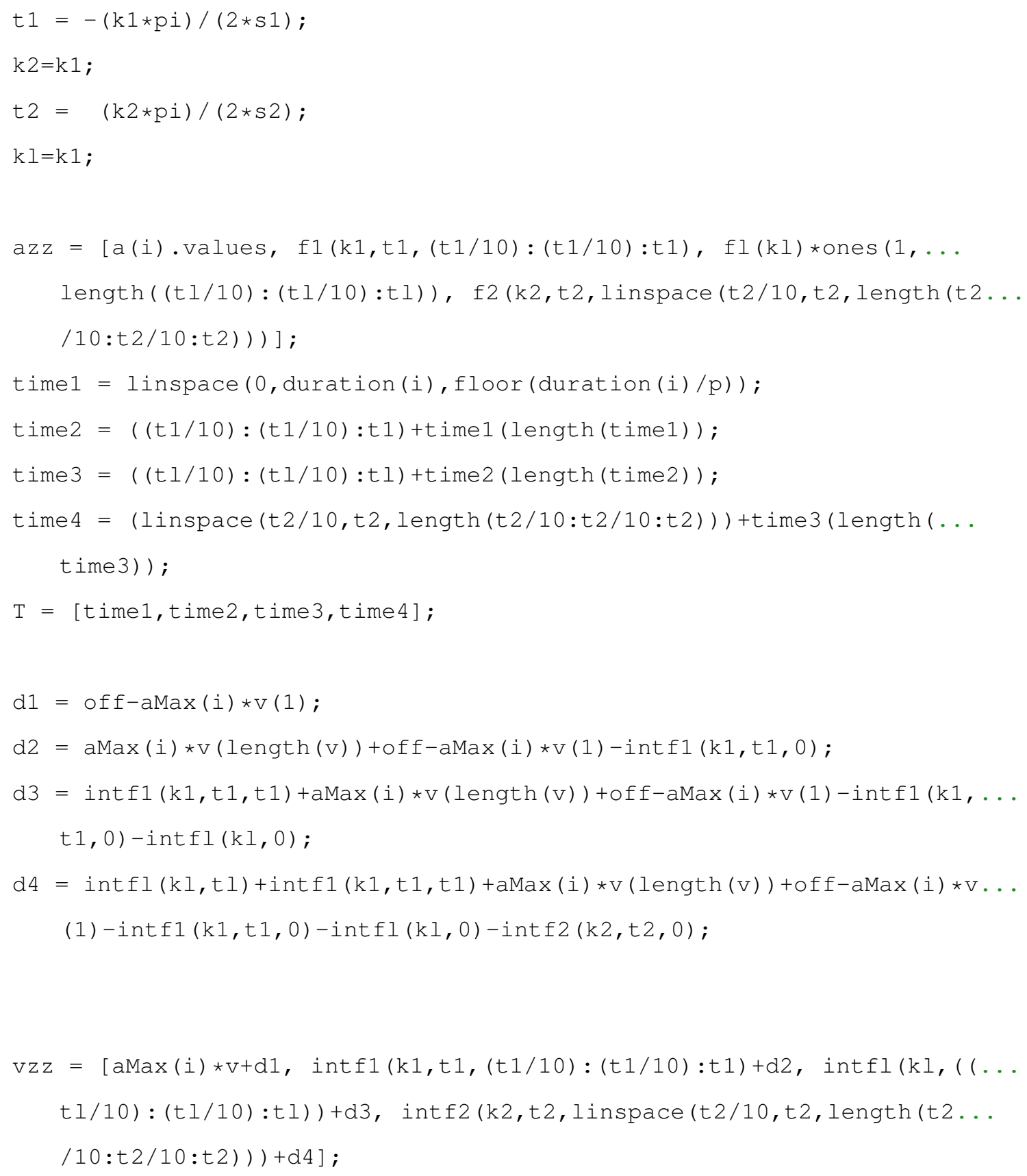




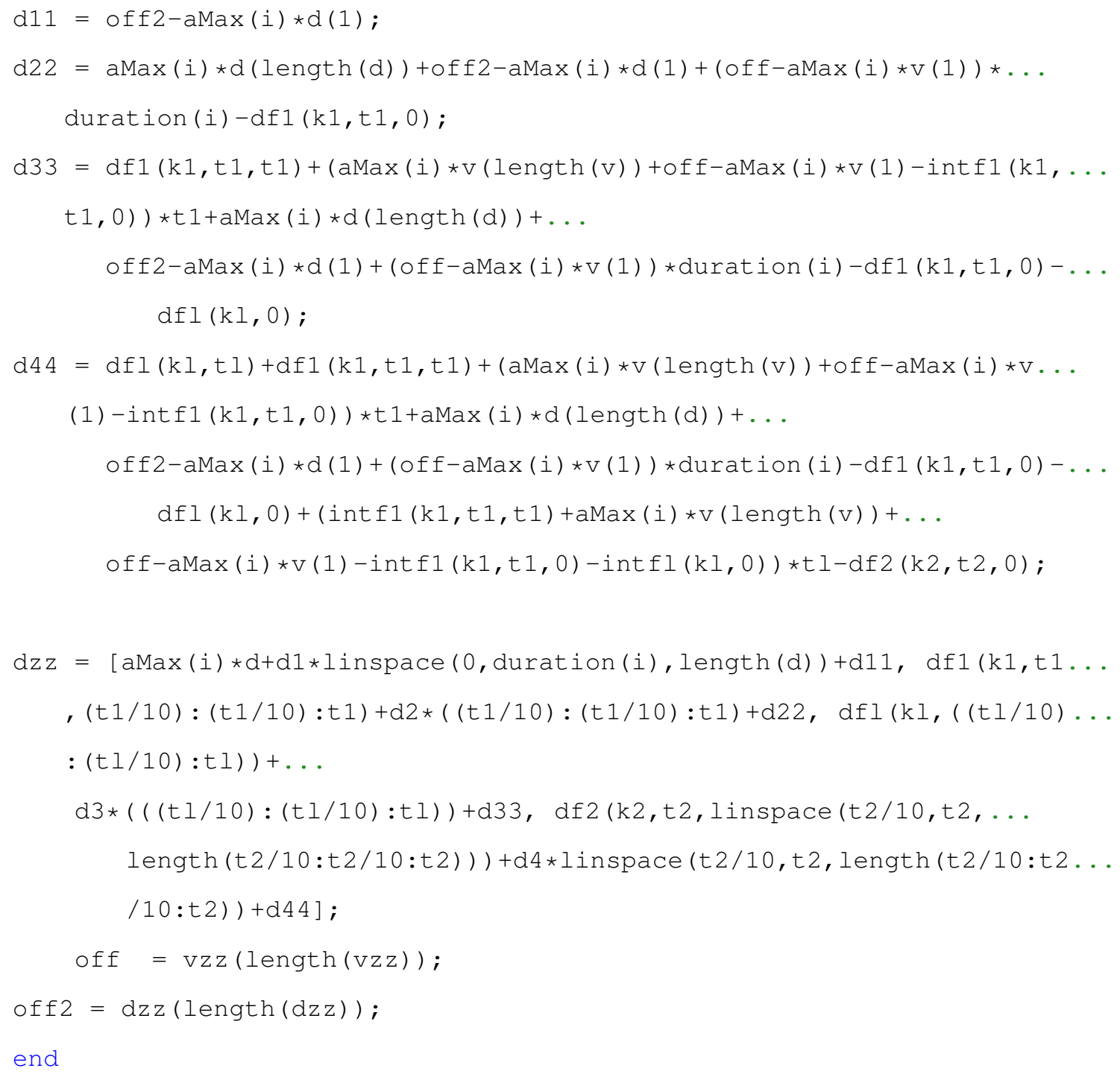




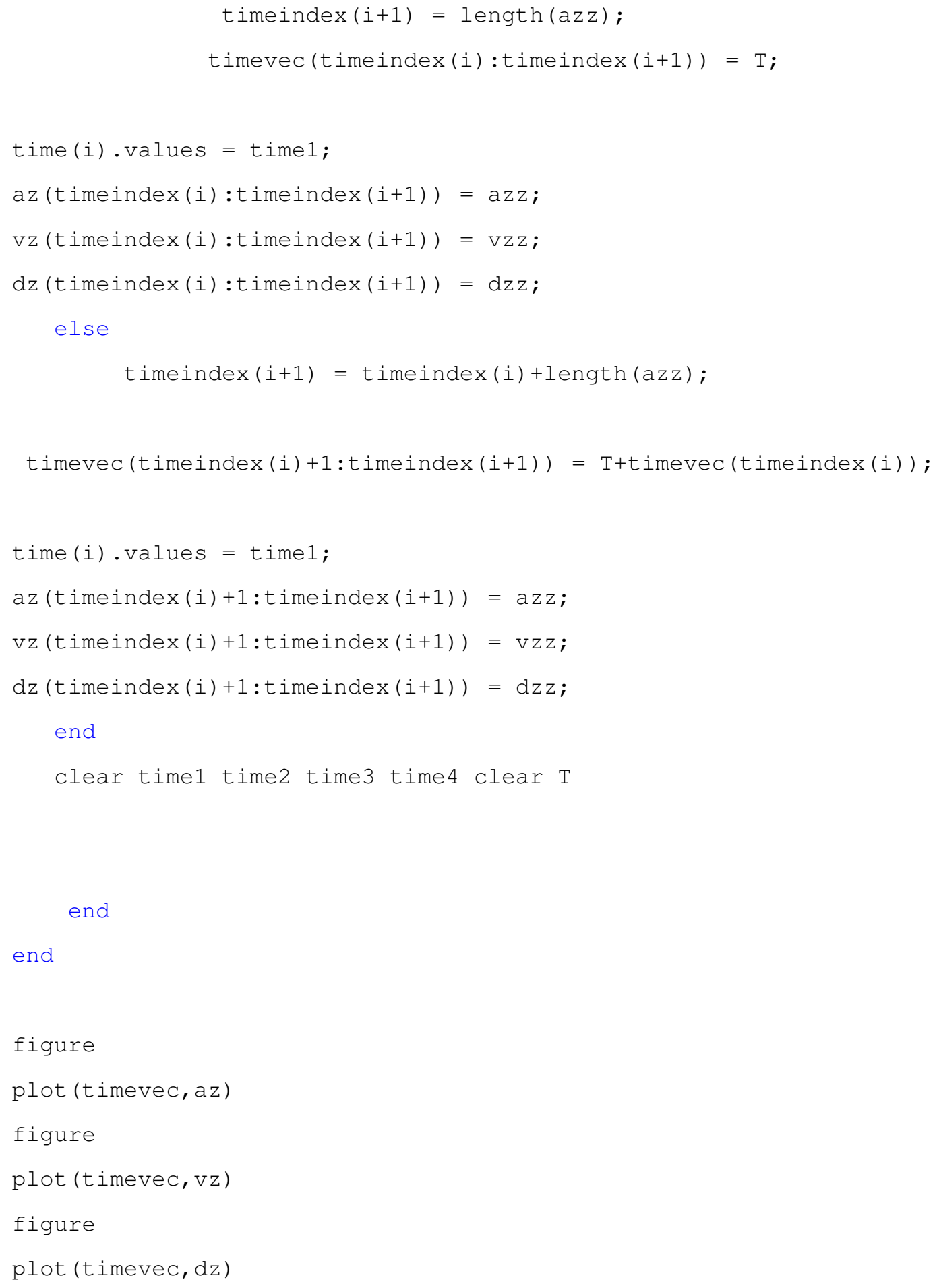


xlabel ('Time (s)')

ylabel ('Acceleration (g' 's)')

generatedpeak = struct ('az', a, 'time', time, 'aMax', aMax, 'duration',...

duration, 'durationtomax', durationtomax, 'ty', ty) ;

end 


\title{
Appendix F
}

\section{Code for Obtaining Parameter Mean}

\author{
Values
}

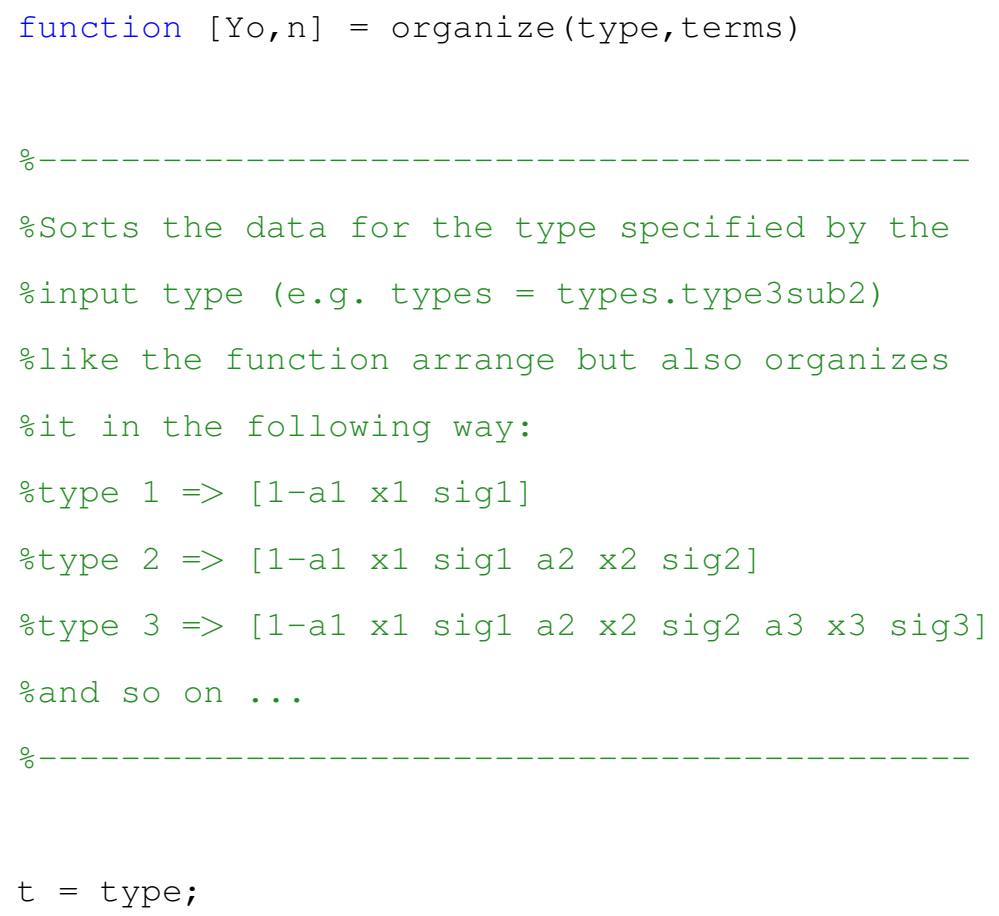




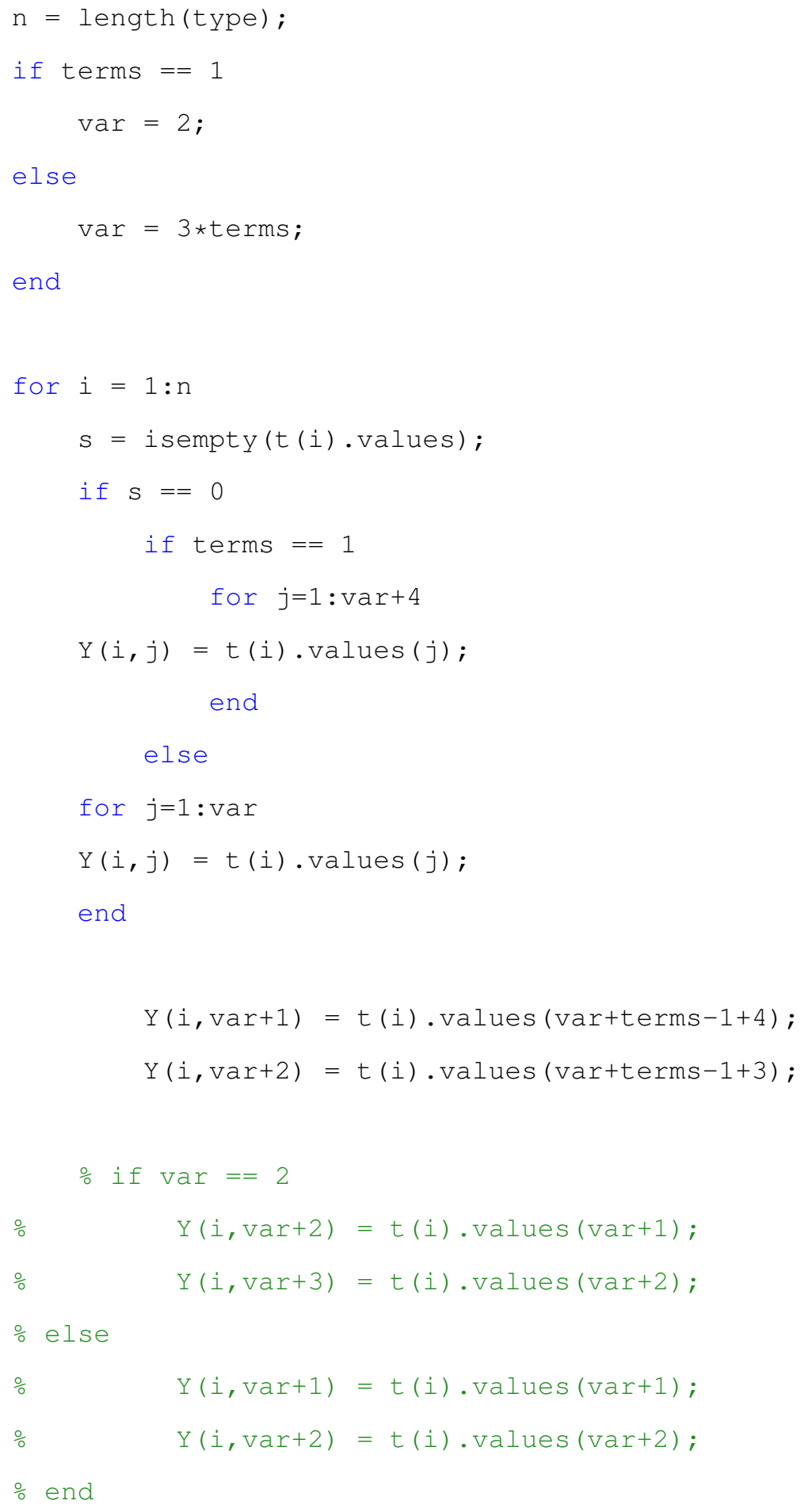




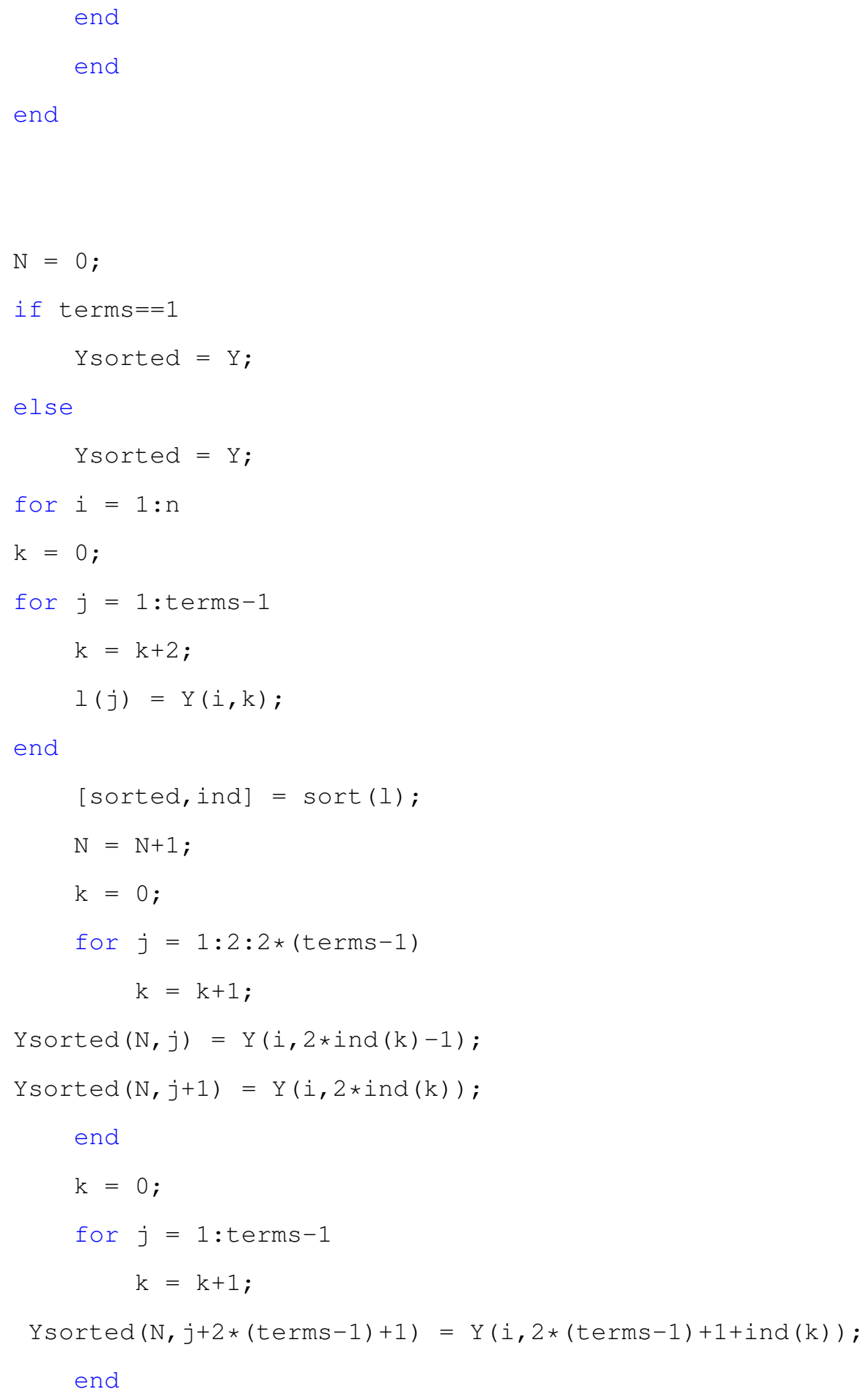




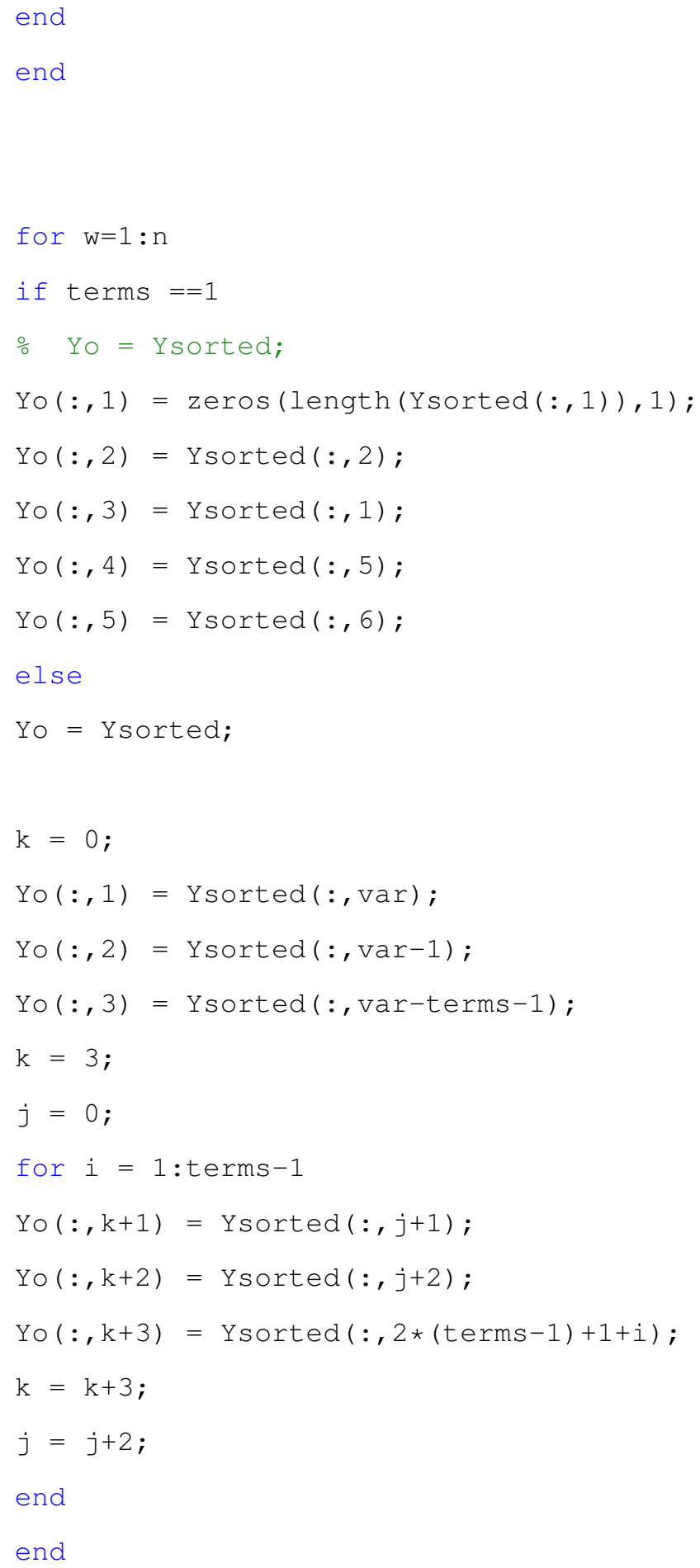


end

end

function [means] = getmeans(types)

$\frac{\circ}{0} \ldots$

oGets the mean values for Types 1 to 4 and outputs in means.

oThe format of means is the same as the tables in the thesis:

$\therefore$ type $1 \Rightarrow$ means $(1,1)=1-a 1, \operatorname{means}(1,2)=x 1, \ldots$

․ type $2 \Rightarrow$ means $(2,1)=1-a 1, \ldots$

$\div$

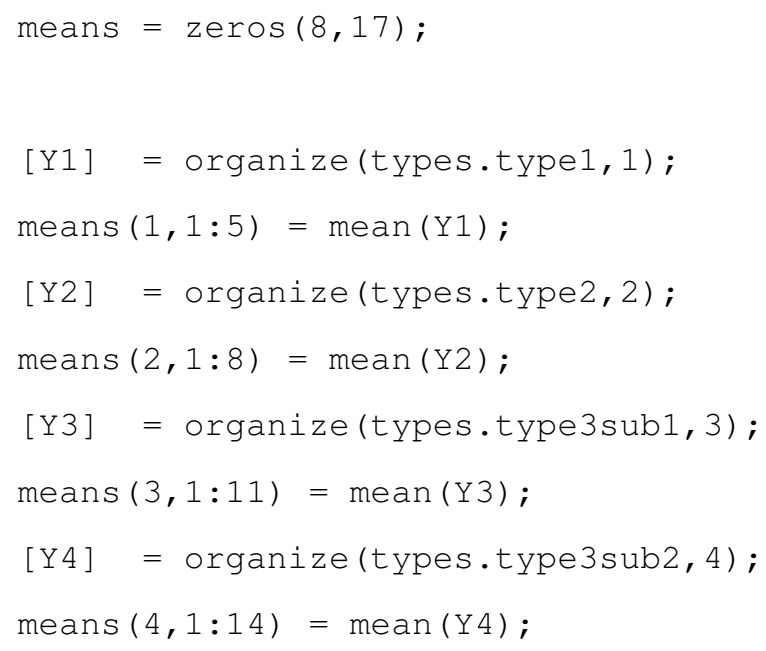




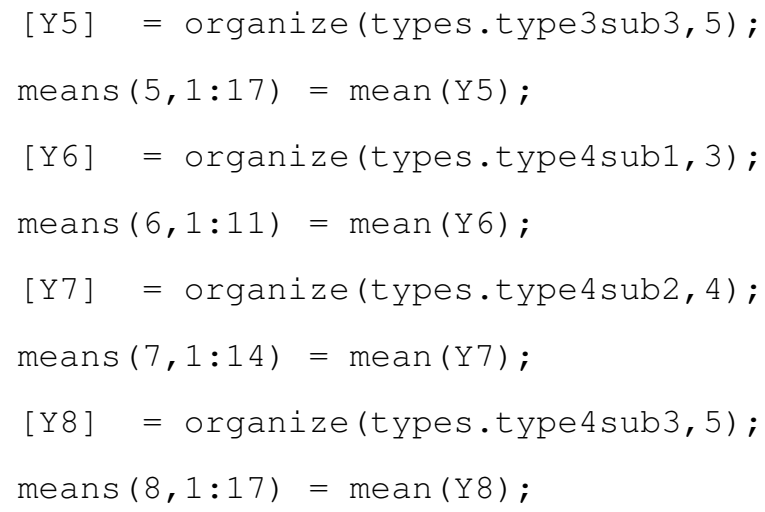

end

function [means] = getmeanstype 5 (types)

$\div$

oGets the mean values for Type 5 pulses and outputs in means.

\% The format of means is the same as the tables in the thesis:

․ype $1=>\operatorname{means}(1,1)=1-a 1, \operatorname{means}(1,2)=x 1, \ldots$

ype $2 \Rightarrow$ means $(2,1)=1-a 1, \ldots$

…

means $=$ zeros $(17,17) ;$

$\operatorname{try}$ 


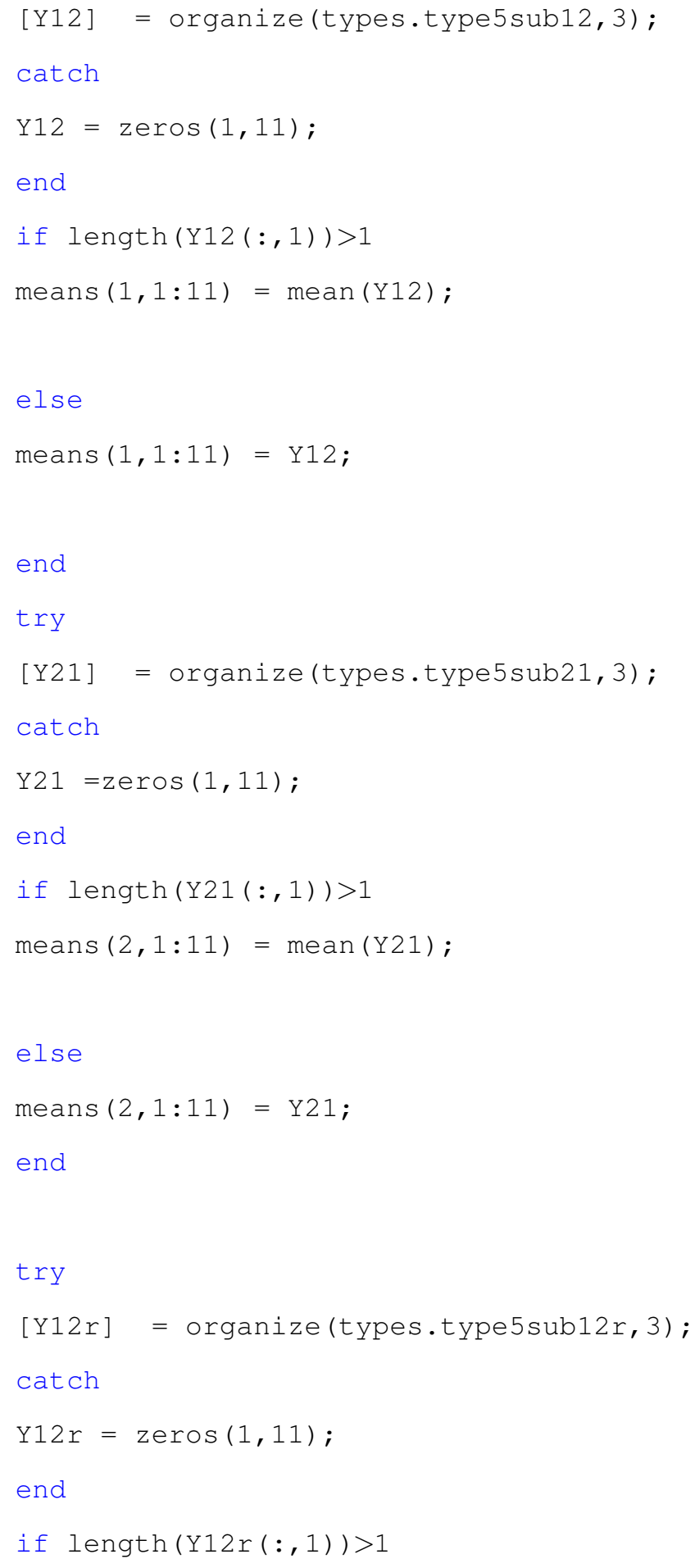




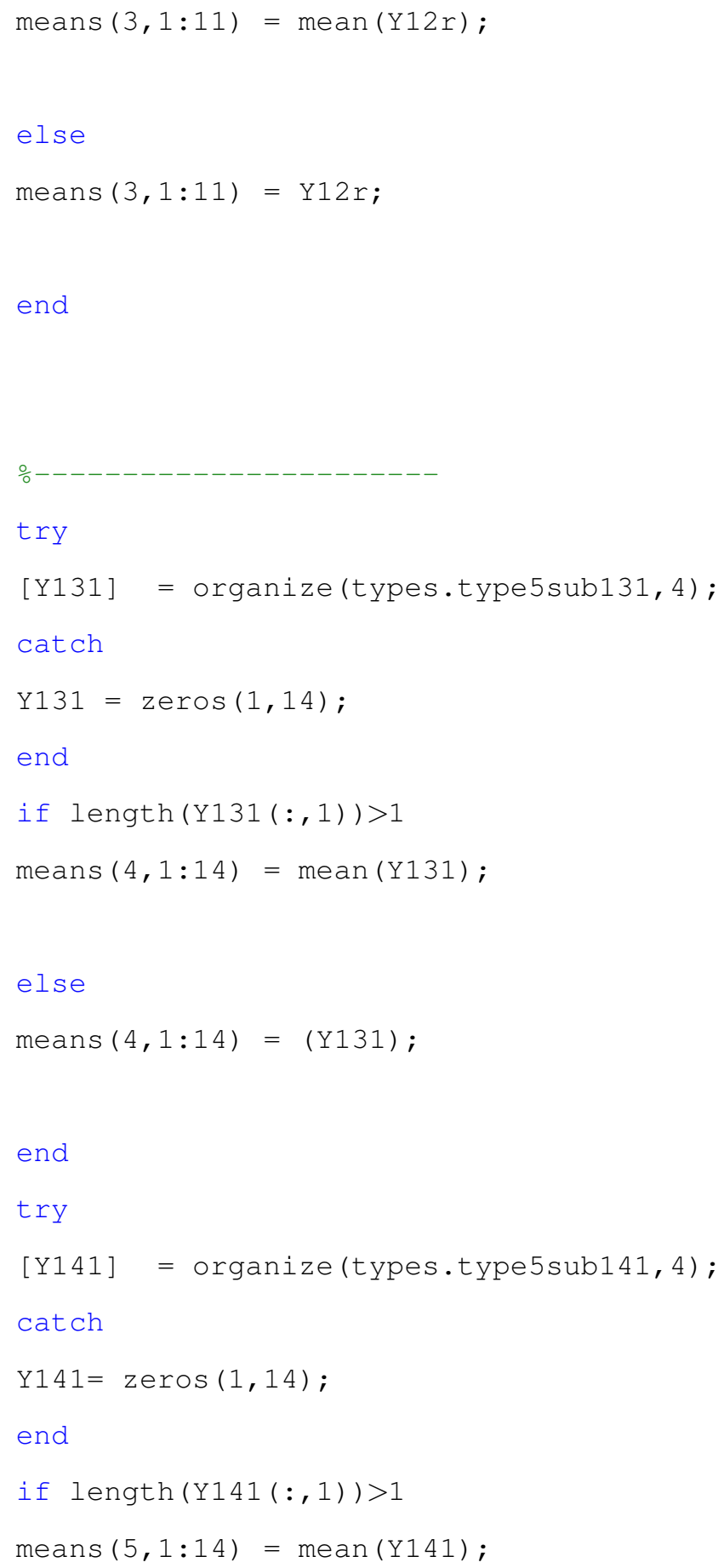




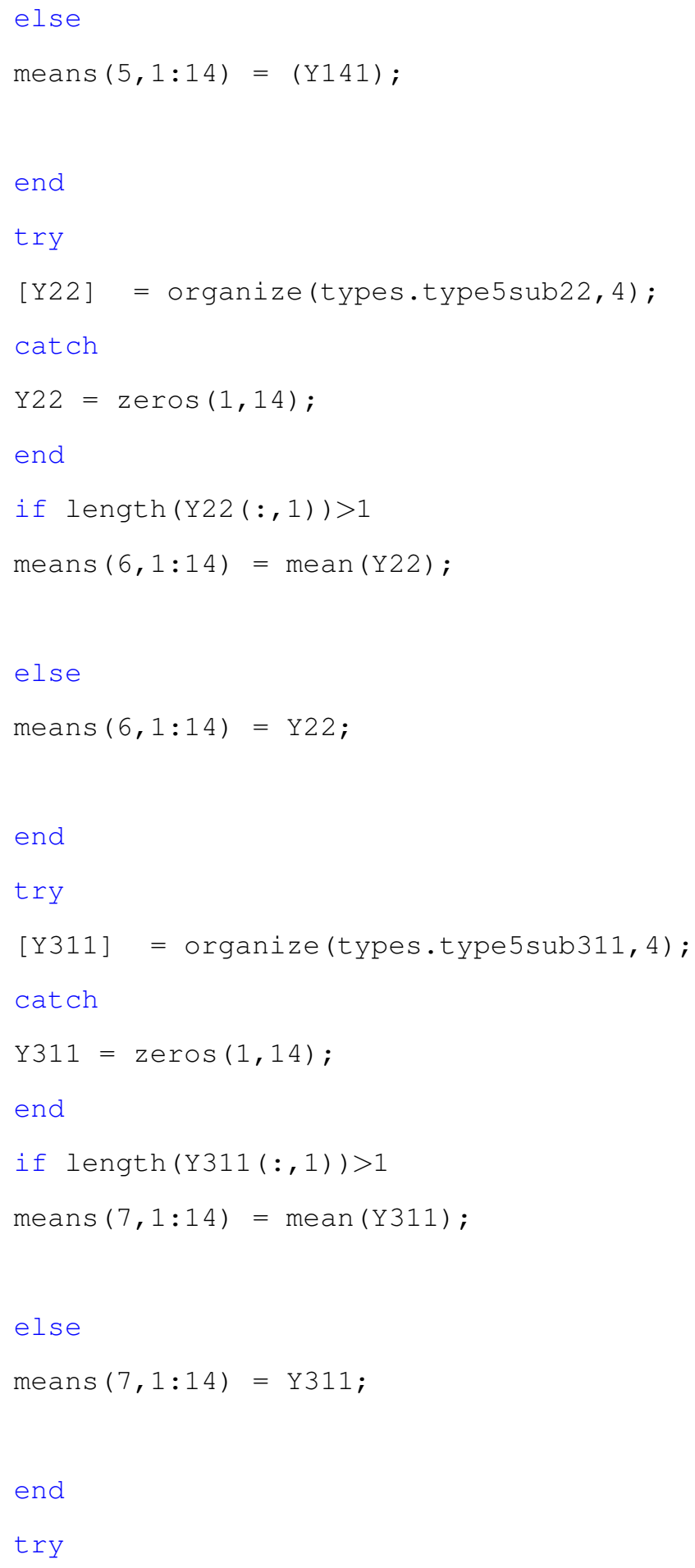




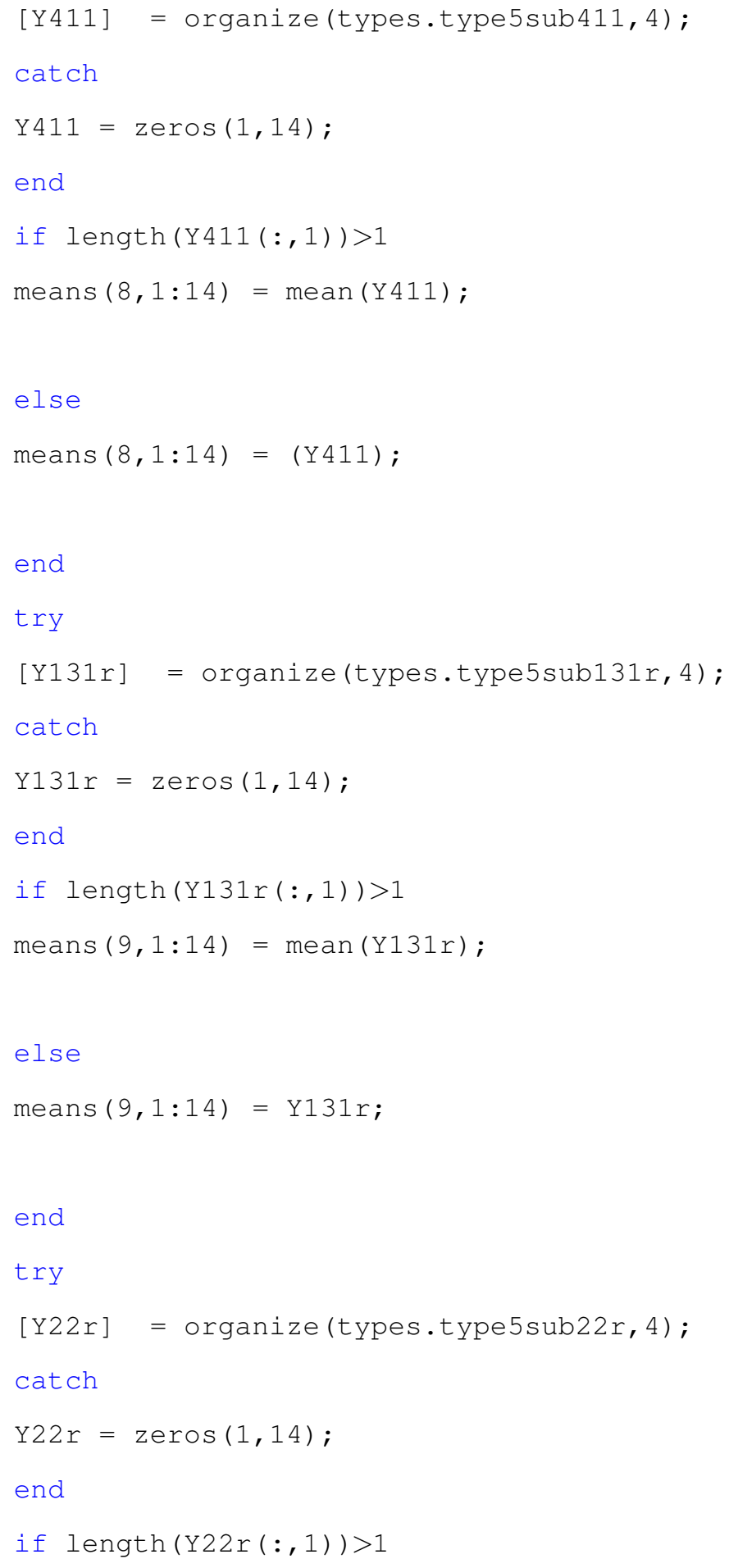




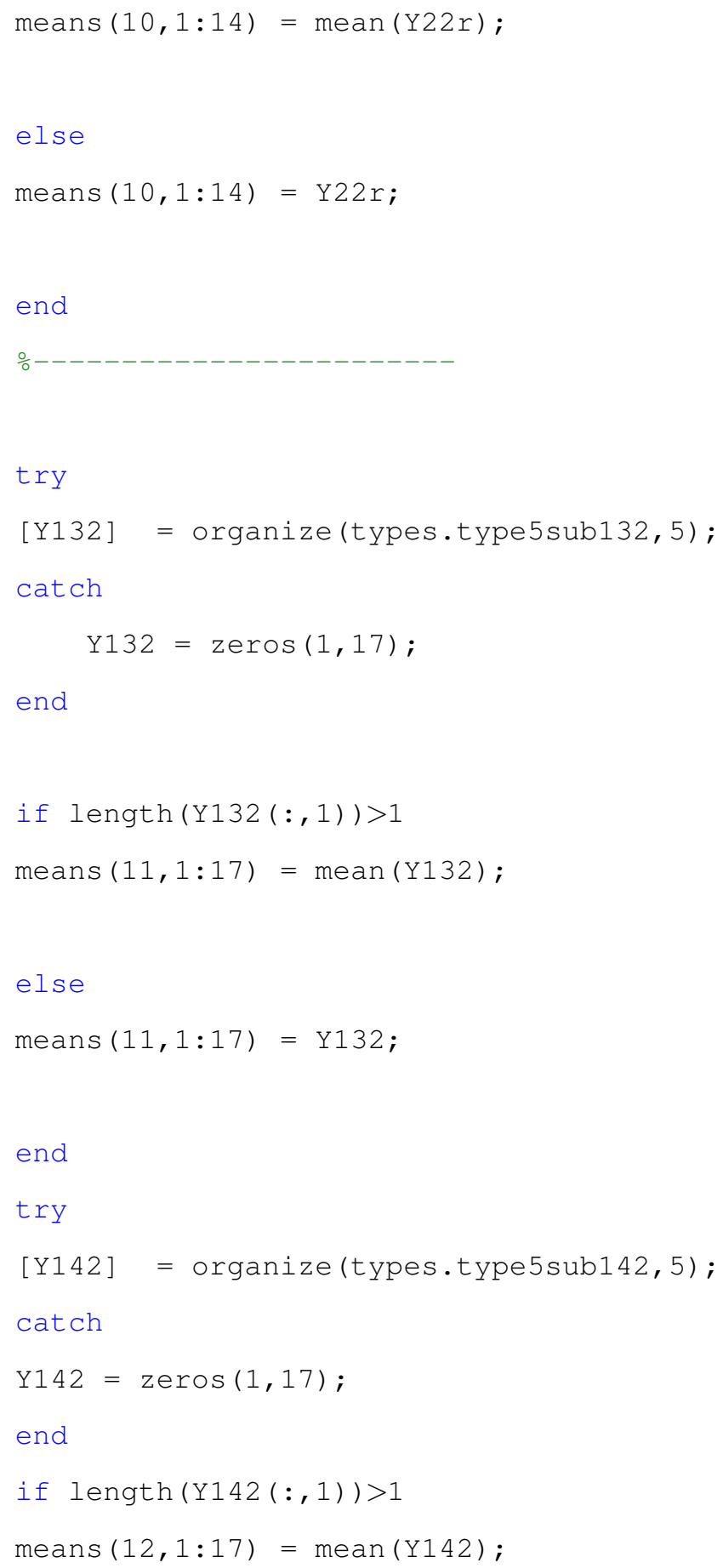




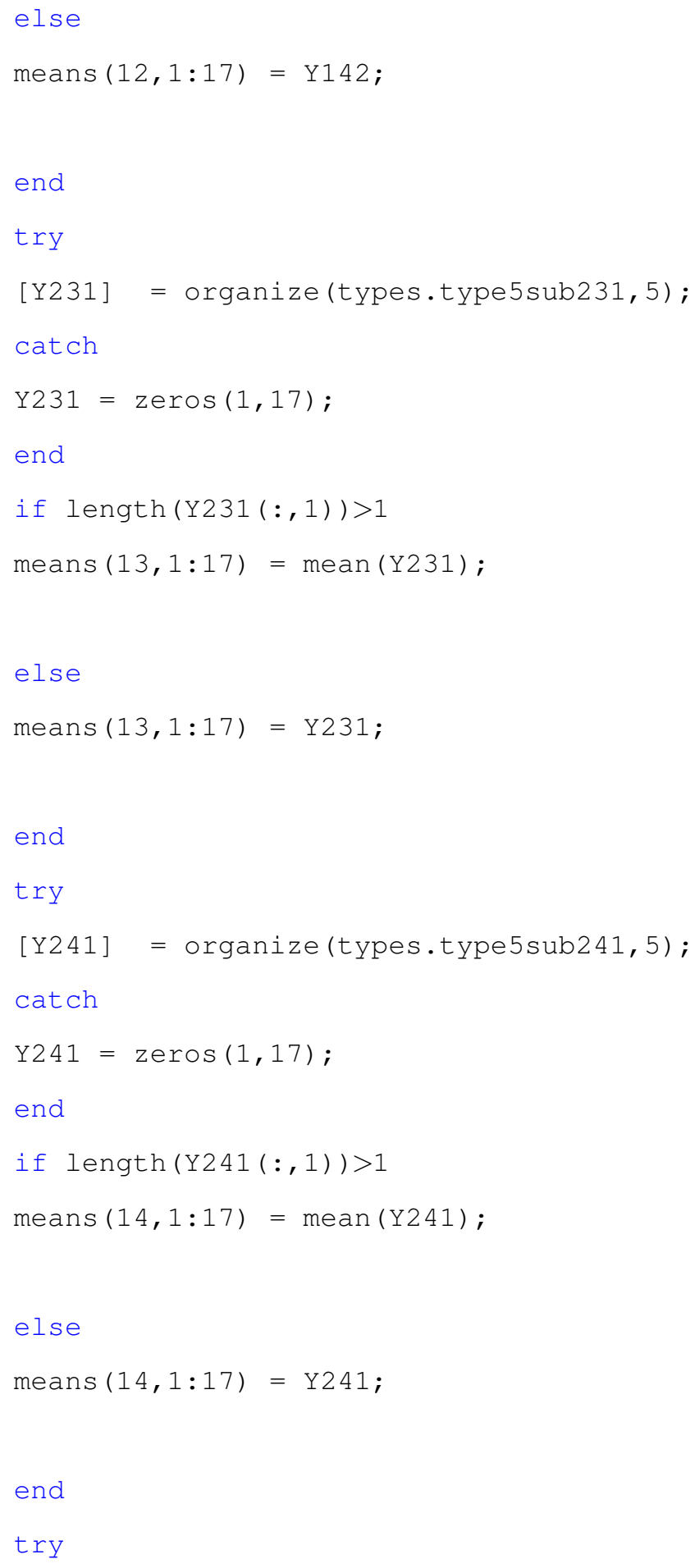




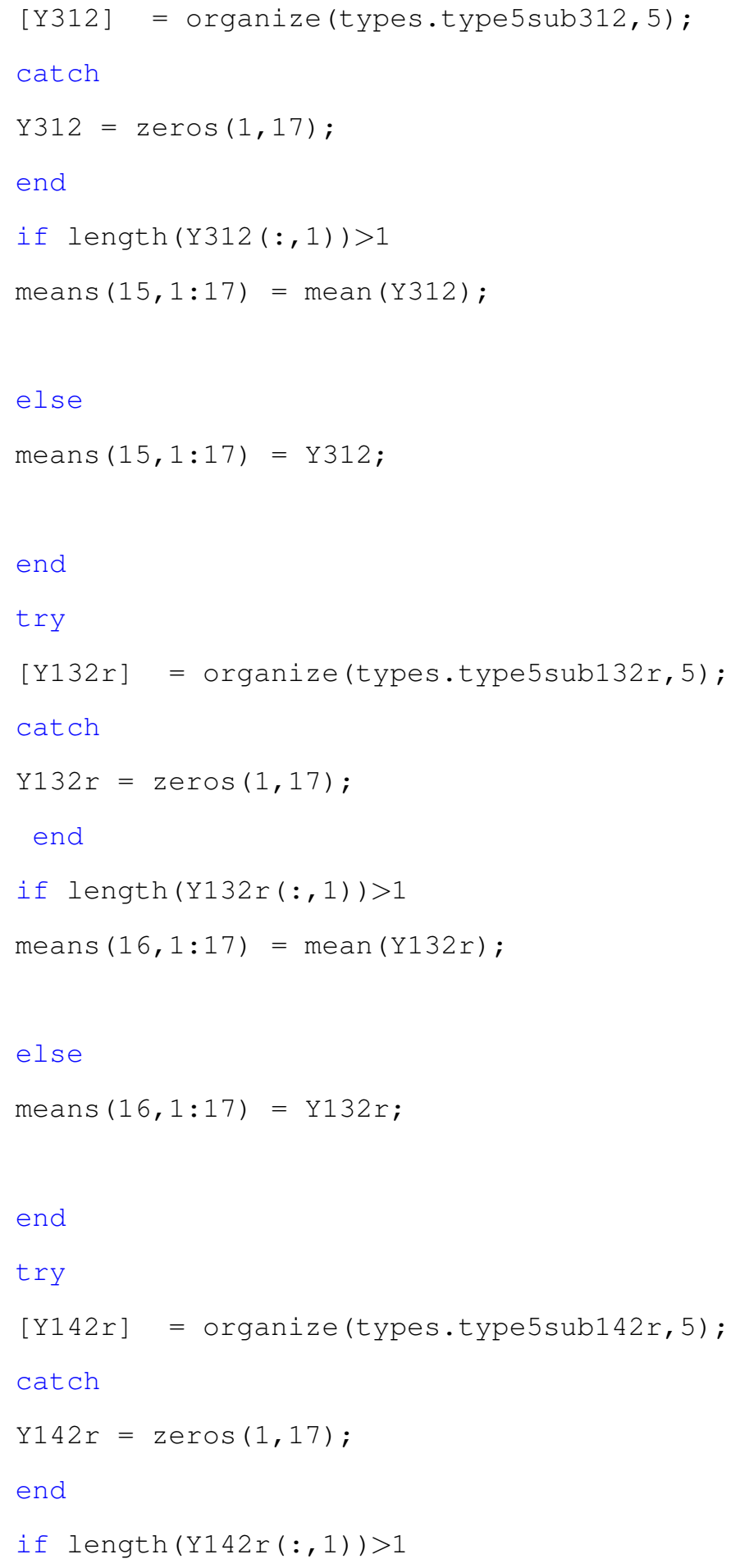


means $(17,1: 17)=\operatorname{mean}(Y 142 r)$;

else

means $(17,1: 17)=Y 142 r ;$

end

end 